\title{
Haemostasis monitoring
}

Citation for published version (APA):

Kuiper, G. J. A. J. M. (2018). Haemostasis monitoring: pinpointing using point-of-care. [Doctoral Thesis, Maastricht University]. Datawyse / Universitaire Pers Maastricht. https://doi.org/10.26481/dis.20180531gk

Document status and date:

Published: 01/01/2018

DOI:

10.26481/dis.20180531gk

Document Version:

Publisher's PDF, also known as Version of record

\section{Please check the document version of this publication:}

- A submitted manuscript is the version of the article upon submission and before peer-review. There can be important differences between the submitted version and the official published version of record.

People interested in the research are advised to contact the author for the final version of the publication, or visit the DOI to the publisher's website.

- The final author version and the galley proof are versions of the publication after peer review.

- The final published version features the final layout of the paper including the volume, issue and page numbers.

Link to publication

\footnotetext{
General rights rights.

- You may freely distribute the URL identifying the publication in the public portal. please follow below link for the End User Agreement:

www.umlib.nl/taverne-license

Take down policy

If you believe that this document breaches copyright please contact us at:

repository@maastrichtuniversity.nl

providing details and we will investigate your claim.
}

Copyright and moral rights for the publications made accessible in the public portal are retained by the authors and/or other copyright owners and it is a condition of accessing publications that users recognise and abide by the legal requirements associated with these

- Users may download and print one copy of any publication from the public portal for the purpose of private study or research.

- You may not further distribute the material or use it for any profit-making activity or commercial gain

If the publication is distributed under the terms of Article $25 \mathrm{fa}$ of the Dutch Copyright Act, indicated by the "Taverne" license above, 
Haemostasis monitoring:

pinpointing using point-of-care 
Printing: Datawyse | Universitaire Pers Maastricht ISBN: 978-94-6295-886-9

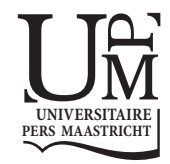

@ C G.J.A.J.M. Kuiper, Maastricht 2018

No part of this publication may be reproduced, stored in a retrieval system or transmitted in any form or by any means, electronic, mechanical, photocopying, recording or otherwise, without the prior written permission of the author. 


\title{
Haemostasis monitoring: pinpointing using point-of-care
}

\author{
Proefschrift
}

ter verkrijging van de graad van doctor aan de Universiteit Maastricht, op gezag van de Rector Magnificus, Prof. dr. Rianne M. Letschert, volgens het besluit van het College van Decanen, in het openbaar te verdedigen op

donderdag 31 mei 2018 om 12:00 uur

door

Gerhardus Johannes Albert Josef Maria Kuiper 
Promotores:

Prof. dr. H. ten Cate

Prof. dr. W.F. Buhre

Copromotores:

Dr. ir. Y.M.C. Henskens

Dr. M.D. Lancé

Beoordelingscommissie:

Prof. dr. J.P. Kooman (voorzitter)

Dr. R.A. Bouwman (CZE, Eindhoven)

Prof. dr. C.D. Dirksen

Prof. dr. J.W.M. Heemskerk

Prof. dr. G.C.M. Kusters (JBZ, 's-Hertogenbosch) 




\section{Table of contents}

Chapter 1 Thesis outline

Chapter 2 Coagulation monitoring in transfusion practice

Chapter 3 The use of regression analysis in determining reference intervals for low haematocrit and thrombocyte count in electrode aggregometry and platelet function analyzer 100 testing of platelet function

Chapter 4 Routine haemostasis testing before transplanted kidney biopsy: a cohort study

Chapter 5 Validation of a modified thromboelastometry approach to detect changes in fibrinolytic activity

Chapter 6 Rapid and correct prediction of thrombocytopenia and hypofibrinogenemia with rotational thromboelastometry in cardiac surgery

Chapter 7 Shifts of transfusion demand in cardiac surgery after implementation of rotational thromboelastometry guided transfusion protocols: analysis of the HEROES-CS (haemostasis registry of patients in cardiac surgery) observational prospective open cohort database

Chapter 8 General discussion and summary

Chapter 9 Valorisation 



\section{Chapter 1}

\section{Thesis outline}





\section{Chapter 1}

\section{Thesis outline}

A prerequisite of adequate treatment of patients with bleeding disorders, is the availability of accurate diagnostic techniques. In particular, in the clinical situation of massive bleeding disorders, in which causality is often multifactorial, prompt diagnosis of the coagulation status is of utmost importance. Point-ofcare devices in haemostasis monitoring enable the clinician to pinpoint the cause and start treatment in a goal-directed manner. However, point-of-care viscoelastic and platelet function test devices have blind spots and pitfalls when managing haemostasis and its monitoring. In this thesis the clinical use of the evolving practice of point-of-care based coagulation monitoring and its effects on transfusion treatment algorithms is outlined.

For a thorough understanding of the basics of coagulation and its monitoring modalities, a detailed overview is given in chapter two. In this chapter the physiology of coagulation is reviewed, followed by a summary how coagulation can be monitored via a classical laboratory approach and by means of point-ofcare techniques. In the final part of this chapter an overview is given of allogeneic blood products and prohaemostatic medication, which are frequently used in daily transfusion practice.

In the third chapter, caveats of two commercially available platelet function testing devices are tackled. We found that for the Multiplate (multiple electrode aggregometry; MEA) as well as the PFA-100 (platelet function analyzer 100) results need to be interpreted in relation to the amount of thrombocytes. In addition, the same results need also to be adjusted for the level of haematocrit in case of the PFA-100. Both findings highlight the need to correctly interpret the results when dealing with anaemic and/or thombocytopenic conditions.

The findings presented in chapter three were used in a subsequent study which is provided in chapter four. Here we investigated the added value of 


\section{Chapter 1}

routine coagulation screening before biopsy of transplanted kidneys. A vast array of tests are investigated, however we were not able to predict bleeding complications, with any of the tests.

In chapter five a newly developed assay for the rotational thromboelastometry (ROTEM) is presented in order to detect hypo- or hyperfibrinolysis. The technical validation was done in healthy volunteers. Consecutively, this method was clinically studied in thrombotic and bleeding pathologies. We examined the fibrinolytical system in patients with sepsis, with cirrhotic liver disease, during pregnancy, and after cardiac surgery. Contrary to the conventional plasma based euglobulin clot lysis time, which seemed to be insensitive to tranexamic acid, our ROTEM-based test was able to pick up the hypofibrinolytical potential after use of tranexamic acid in cardiac surgery patients.

In the sixth chapter the advantages of using a point-of-care device like ROTEM in a quick decission setting like cardiac surgery are studied and discussed. We observed that the clinical use of ROTEM enables the physician to detect states of hypofibrinogenemia and thrombocytopenia. The results needed for the initiation of treatment were found to be available much earlier than by means of conventional laboratory tests.

The added value of implementation of a ROTEM-based transfusion practice in the field of cardiac surgery is outlined in chapter seven. This chapter provides the results of a large prospective study which showed that the use of a point-ofcare approach for coagulation monitoring with ROTEM, resulted in a reduction of allogeneic transfusional products, of the incidence of rethoracotomies, and of the length of stay, both at the intensive care unit and in the hospital. Moreover, point of care monitoring and application of the diagnostic parameters leads to a significant reduction in costs per patient.

In chapter eight the whole thesis is discussed and a summary is given. The findings of the studies are being highlighted and evaluated with respect to avail- 
able knowledge. In addition, a brief look into future monitoring modalities is given in this chapter, while focussing on potential new research questions.

In chapter nine the bigger picture of this thesis is being emphasized. In this valorisation chapter, potential interests and broader values to society of the study findings are unfolded.

The thesis ends with a word of acknowledgement to contributors to this thesis, a short curriculum vitae, and an overview of other scientific output (chapters ten, eleven, and twelve). 



\section{Chapter 2}

\section{Coagulation monitoring and} transfusion practice

Kuiper GJ, ten Cate H.

Section 11: the hematological system, part 11.1: laboratory monitoring, chapter 266: coagulation monitoring. Oxford Textbook Of Critical Care, Second Edition, May 2016, ISBN 9780199600830.

(Published in part) 



\section{Chapter 2}

\section{Coagulation monitoring and transfusion practice}

In this introductory chapter the practice of coagulation monitoring will be outlined. First of all an overview of haemostasis will be given. After that, we will be delving into the monitoring practice of haemostasis. In the final part frequent treatment modalities will be reviewed which are being used in transfusion practice.

Haemostasis: platelets and the coagulation and fibrinolysis cascade Haemostasis is a dynamic process between blood constituents and the vessel wall required to stop bleeding after vessel wall damage (figure 2.1). The involvement of platelets is classically described as primary haemostasis, while the coagulation proteins are responsible for secondary haemostasis. The breakdown of a blood clot is mediated by so-called fibrinolysis. ${ }^{1}$

Platelets have several receptors for the activation of platelets, the adherence to collagen, and the aggregation with other platelets. In vivo, vessel wall damage exposes collagen to platelets, to which they can adhere through glycoproteins including the GP lb receptor with von Willebrand factor (vWF) as a co-factor. Other activators are thrombin, arachidonic acid, epinephrine, and adenosine diphosphate (ADP). Upon activation, platelets aggregate via GP Ilb/IIla receptors with other platelets through fibrinogen and vWF.

Vessel wall damage also exposes tissue factor (TF). Coagulation factor VII (FVII) is activated by TF and this complex can activate FX, with FV as its cofactor, in order to convert prothrombin into thrombin. This is classically described as the extrinsic route. Activation of the intrinsic route is achieved by contact activation via negatively charged surfaces in vitro and by compounds like polyphosphates in vivo. Activated FXII starts an intrinsic cascade through activation of FXI, which yields activated FIX. FIXa, with FVIIla as its cofactor, activates FX and this again leads to prothrombin conversion. Thrombin stimulates fibrin and FXIIla formation, which stabilizes fibrin via cross-links. Thrombin as the central 
coagulation enzyme has pro-, as well as anticoagulant properties, thereby balancing the haemostatic system. Thombomodulin (TM), an endothelial receptor, binds thrombin and hereby inactivates thrombin's procoagulant properties. Protein $\mathrm{C}$, activated via this thrombin-thrombomodulin complex, can inactivate $\mathrm{FVa}$ and FVIIla. Circulating antithrombin attenuates thrombin formation by neutralizing FXa and thrombin, while tissue factor pathway inhibitor (TFPI) inactivates FXa when in complex with TF-FVIIa.

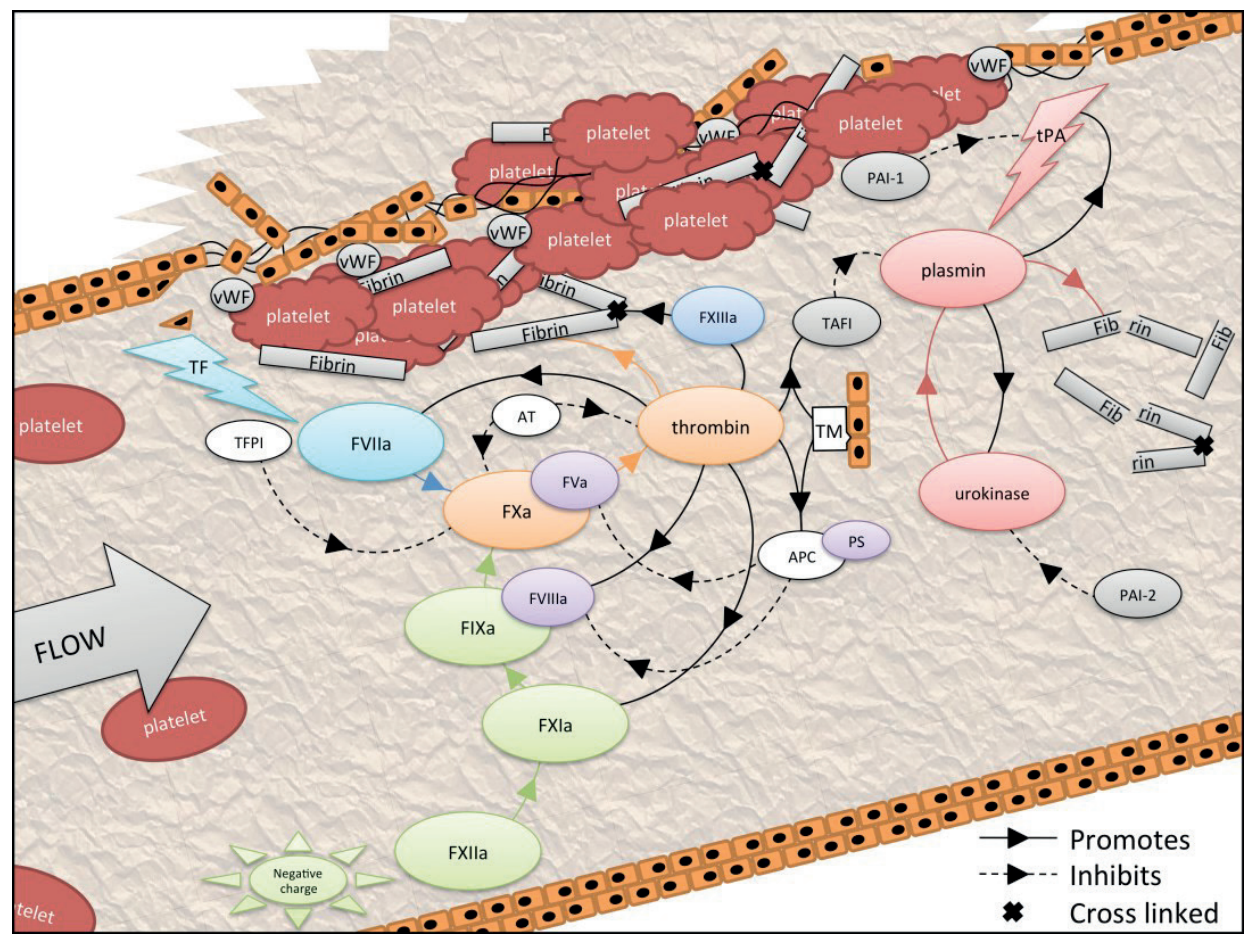

Figure 2.1 Simplified graphical representation of coagulation in vivo.

Pathways: Light blue, extrinsic pathway; green, intrinsic pathway; orange, common pathway; red, fibrinolytic pathway; white, anticoagulant proteins; purple, cofactors; dark blue, fibrin cross-linking by FXIIla; black lines and arrows, feedback loops.

Abbreviations: APC, activated protein C; AT, antithrombin; F[\#]a, activated factor [\#]; PAI-1/2, plasminogen activator inhibitor 1/2; PS, protein S; TAFI, thrombin activatable fibrinolysis inhibitor; TF, tissue factor; TFPI, tissue factor pathway inhibitor; TM, thrombomodulin; tPA, tissue type plasminogen activator; vWF, von Willebrand factor.

The fibrin mesh is degraded by plasmin, resulting from the action of tissue-type plasminogen activator (tPA), released from endothelial cells, on plasminogen. Likewise, urokinase is a potent fibrinolysis activator. Thrombin inhibits fibrinoly- 
sis via thrombin activatable fibrinolysis inhibitor (TAFI) when bound to TM, while platelets secrete plasminogen activator inhibitor (PAI)-1 and 2, which inhibit tPA and urokinase, respectively. Fibrin is degraded into fragments called fibrin degradation products (FDP's). D-dimer is an FDP in which the cross-link is still present.

\section{Classical Coagulation Monitoring}

In primary haemostasis testing various laboratory assays are adopted to reflect the function of platelets. In short, for effective primary haemostasis, a sufficient number of functional platelets need to be present in the body. First of all, a platelet count is indispensable, however, this does not adequately reflect the function of the respective platelets. Mean platelet volume (MPV) gives information on the volume of the platelets, reflecting the turnover of platelets and their reactivity. A high MPV could indicate a high platelet turnover due to increased production of platelets from bone marrow-derived megakaryocytes. A low MPV is usually an indication of less reactive platelets or due to splenic sequestration. The gold standard in platelet function testing is light transmission aggregometry (LTA). The test needs, however, a sufficient number of platelets to be reliable, while interpretation of the results is merely based on expertise, rather than specific cut-off values. The bleeding time test (the Ivy method), being the only in vivo platelet function test, is poorly reproducible. A prolonged bleeding time could indicate a platelet dysfunction. However, a normal test does not rule out a platelet disorder.

For monitoring secondary haemostasis the prothrombin time (PT), activated partial thromboplastin time (aPTT), thrombin time (TT), and reptilase time (RT) reflect disorders in specific steps of the coagulation cascade. The PT is activated by adding TF, the aPTT by a contact activator. The international normalized ratio (INR) is a ratio of the PT of the local laboratory compared with a standardized reference PT. Prolonged PT and APTT test are normally accompanied by a bleeding tendency (table 2.1), although the presence of the lupus anticoagulant, which is an antibody, is paradoxically associated with a thrombotic tendency. An aPTT prolongation due to FXII deficiency does not exert in a bleeding or throm- 
botic tendency. The TT is measured by directly adding thrombin to plasma, which triggers fibrin formation. The TT is thus sensitive to fibrinogen, but also to FDP's (which themselves inhibit fibrin polymerization) and to heparin therapy. RT is similar to TT, but insensitive to heparin. Lastly, levels and activities can also be measured for each separate coagulation factor. Fibrinolysis is frequently monitored by measuring D-dimer levels (or FDP's), fibrinogen levels, and the euglobulin clot lysis time. Excessive clot breakdown will result in low fibrinogen levels and high D-dimer or FDP levels. D-dimer has a low specificity, thereby making it only suitable to exclude (ongoing) fibrinolysis for the diagnosis in patients. Fibrinogen, as an acute phase protein, can be elevated in a wide variety of diseases. The euglobulin clot lysis time is an elaborate test in which the overall lysis potential of a formed clot can be quantified. A shortened euglobulin clot lysis time is an indication of potential hyperfibrinolysis.

Table 2.1 Causes of PT and aPTT test abnormalities

\begin{tabular}{lll}
\hline PT & aPTT & Possible cause \\
\hline \multirow{2}{*}{ Prolonged } & Normal & $\begin{array}{l}\text { FVII deficiency or inhibitor } \\
\text { Vitamin K deficiency or vitamin K antagonist therapy } \\
\text { Liver disease }\end{array}$ \\
\hline \multirow{2}{*}{ Normal } & Prolonged & $\begin{array}{l}\text { FVIII, FIX, and FXI deficiencies or inhibitors (haemophilia A/B/C) } \\
\text { FXII, prekallikrein, or HMW kininogen deficiencies or FXII inhibitor } \\
\text { Von Willebrand disease } \\
\text { Heparin therapy } \\
\text { Lupus anticoagulant }\end{array}$ \\
\hline \multirow{2}{*}{ Prolonged } & Prolonged & $\begin{array}{l}\text { FX, FV, prothrombin, and fibrinogen deficiencies or inhibitors } \\
\text { Disseminated intravascular coagulation, trauma-induced coagulopathy } \\
\text { Combined VKA and heparin therapy }\end{array}$ \\
\hline
\end{tabular}

\section{Point-of-care Coagulation Monitoring}

Point-of-care monitoring (POC) provides simple, rapid bedside testing for various laboratory tests. In coagulation monitoring the activated clotting time (ACT), a viscoelasticity measuring technique (TEG, ROTEM, or Sonoclot systems), and platelet function tests are widely-used, whole blood tests for coagulation monitoring.

In the ACT test, native whole blood is activated via the intrinsic pathway by either kaolin or celite. The time to form a clot is the ACT given in seconds. The ACT is used in cardiothoracic surgery to monitor unfractionated heparin therapy and its reversal by protamine sulphate. The ACT is less sensitive to therapy with low molecular weight heparins and insensitive to pentasaccharide therapy. ${ }^{2}$ 
The TEG (thrombelastography) and ROTEM (rotational thromboelastometry) systems are the most widely used whole blood viscoelasticity devices, together with the Sonoclot. ${ }^{3}$ The basic principle is that citrate anticoagulated whole blood is activated after recalcification with either an extrinsic or intrinsic activator in a measuring cuvette. While the blood starts to clot, the device measures the strength of the clot formed and plots the strength at each point in time in a graph. Each device has its own parameters, but all three display the time till the fibrin clot starts to form, the maximum clot strength achieved, and the time it takes to dissolve the formed clot. ${ }^{3}$ For instance, the maximum clot strength is the resultant of both platelets and fibrin involved in the formed clot. The decline of the maximum clot strength is again influenced by both platelets (clot retraction) and fibrin (fibrinolysis). ${ }^{4}$ Each system has its own specific measuring principle, its own nomenclature, and (dis)advantages compared with the other two. The consequence is that, thus far, results cannot be interchanged between systems. ${ }^{3,5}$ Correlations between some viscoelasticity parameters and standard coagulation tests are fairly good, making the need for standard coagulation tests redundant in certain scenarios. ${ }^{3}$

Other POC tests are specially developed for platelet function measuring in whole blood. ${ }^{6}$ All these operate according to the same basic principle: whole blood is put in a measuring cuvette and the platelet receptors are then activated by a reagent, aggregating the platelets. This alteration of the platelets is detected by the device. However, normal haematocrit and platelet levels are imperative for platelet function testing either by POC or LTA. ${ }^{7}$

\section{Coagulation Monitoring in Critical Care Diseases}

Coagulation disorders seen in critical care are classically divided into bleeding, thrombosis, or a combination. Monitoring of the three disorders is highlighted in the following paragraphs.

Trauma-induced coagulopathy (TIC) is often referred to as consisting of a lethal triad. Massively bleeding trauma patients present with hypothermia and acidosis. Fluid resuscitation leads to a dilutional coagulopathy completing the triad. ${ }^{8}$ This is further aggravated by endothelial damage, releasing IPA and thrombo- 


\section{Chapter 2}

modulin in abundance, resulting in anticoagulation and hyperfibrinolysis. ${ }^{9}$ Classic laboratory coagulation tests for TIC include PT, aPTT, platelet count, and fibrinogen levels. The first coagulation factor to fall below a critical value is fibrinogen. ${ }^{8}$ In colloid use fibrinogen levels are overestimated and platelet function is impaired. ${ }^{10}$ Low platelet counts in TIC hinder platelet function testing. The use of viscoelasticity measurements in trauma or massive transfusion cases is advocated by many, although evidence for a favourable outcome is thus far scarce. $^{11}$

A mixed bleeding-thrombosis condition is DIC (disseminated intravascular coagulation). In overt DIC excess thrombin is produced, resulting in microvascular thrombosis due to failure of the natural anticoagulant mechanisms. This condition can be accompanied by bleeding due to a depletion of coagulation proteins and platelets. ${ }^{12}$ In the early phase of DIC, fibrinolysis is often impaired due to high PAI-1 levels, while hyperfibrinolysis is present in late DIC. The diagnosis is based on classical coagulation tests incorporated in a scoring system. ${ }^{13}$ Subsequent monitoring should focus on platelet levels and function over time, on the fibrinogen levels, and PT during DIC. Viscoelasticity measurements could help in monitoring hypo- or hyperfibrinolysis.

Haemolysis, elevated liver enzymes and low platelets (HELLP) is a syndrome complicating around $0.5 \%$ of all pregnancies. Monitoring for the presence of DIC and MPV, as a marker for platelet function and consumption, together with platelet levels and POC platelet function testing is advocated. ${ }^{14}$

\section{Coagulation Monitoring in Medication Use}

Antiplatelet and (oral) anticoagulant drugs are frequently used on the ICU or by patients at home. The effect of antiplatelet drugs can be monitored with platelet function tests, either by LTA or POC. ${ }^{6}$ Vitamin $\mathrm{K}$ antagonist monitoring and dose-adjustment is done via the INR. Heparin treatment can be monitored by measuring anti-FXa levels for any type of heparin including pentasaccharides, while aPTT is only suitable in unfractionated heparin use. ${ }^{2}$ A complication of heparin administration is HIT (heparin-induced thrombocytopenia). In HIT suspected patients, the $4 \mathrm{~T}$ 's scoring system followed by specialized laboratory 
analysis is applied. ${ }^{15} \mathrm{New}$ oral anticoagulants (NOACs) are emerging on the market targeted at inhibiting thrombin or FXa. It is advocated that monitoring is unnecessary, although in clinical practice there is growing demand for laboratory tests to monitor these NOACs, certainly in critical situations. ${ }^{16}$ For screening in acute situations, some of the conventional coagulation tests may still be useful, provided that the reagents are sufficiently sensitive. For quantitative measurement, specific anti-FXa-based chromogenic assays (Xa-directed NOACs) or thrombin sensitive clotting methods (for thrombin-directed NOAC) should be used. ${ }^{17}$

In TIC and in the peri-operative setting prohaemostatic therapy can be given in many forms. ${ }^{18}$ Monitoring of prohaemostatic therapy is focused on platelet levels and function, fibrinogen levels, classical coagulation tests, and viscoelasticity measurements. Fibrinogen levels can decline rapidly due to thrombolytic therapy in stroke or venous embolism, resulting in an acquired bleeding tendency.

\section{Transfusion products}

Several products exist which interfere with coagulation either with the purpose to result in anticoagulation or to enhance coagulation. ${ }^{18}$ In bleeding scenarios transfusion products can be subdivided in allogeneic blood products and prohaemostatic medication.

Allogeneic leucocyte depleted transfusion products are gathered from healthy blood donors. The most well-known product is the red blood cells concentrate (RBC). Another blood derived product is the platelet concentrates (PC). Although fresh frozen plasma (FFP) is a split product of whole blood like the two other allogeneic transfusion products which both can be stored for short time only, FFP's can be saved up to 4 years. Transfusion of RBC's serves the purpose of enhancing the oxygen carriage capacity of blood in anaemic conditions. However, there are other noticeable favourable effects of red blood cells on coagulation. One of the haematocrit dependent effects is pushing the smaller platelets towards the outer layer of the bloodstream in laminar flow conditions, hereby facilitating platelet adherence to the damaged vessel wall. ${ }^{19}$ However, 


\section{Chapter 2}

too high haematocrit results in sluggish or halted blood flow and leads to thrombotic complications, while too low haematocrit seems to impair the platelet pushing by red blood cells and thus such patients exhibit a bleeding state. The main constituents of FFP's are the blood proteins. Its use is to supply coagulations proteins when patients are running low in case of vitamin K-antagonist use or due to dilution and consumptions in bleeding cases. PC's are derived from the buffy coats of donated blood. Indications for PC's are thrombocytopenia and bleeding due to thrombocytopathy (either hereditary or acquired forms).

Examples of prohaemostatic medication used in transfusion practice are desmopressin, purified coagulation factor concentrates (like PCC (a prothrombin complex concentrate existing of factors II, VII, IX, and X), or single factors like recombinant activated FVII, FVIII, FIX, and VWF), antifibrinolytics (tranexamic acid, the discontinued aprotinin, and $\varepsilon$-aminocaproic acid), protamine, and calcium. Desmopressin or DDAVP (1-desamino-8-D-arginine vasopressin), a synthetic analogue of vasopressin, exerts its prohaemostatic effects through the release of VWF and FVIII from endothelial Weibel-Palade bodies. ${ }^{20}$ Keep in mind, the fibrinolytically active tPA is also being released by DDAVP. Indications for DDAVP are certain platelet disorders (hereditary and acquired platelet dysfunction disorders caused by medication or uraemia), vWD (von Willebrand disease), and haemophilia A. Almost every coagulation factor can be given as a single purified concentrate to patients. These factor concentrates present a much lower volume needed to administer, hereby reducing overload. Frequently, fibrinogen concentrates together with PCC are used in massive consumptive bleeding due to trauma. Other indication for PCC is reversal of vitamin Kantagonists which is preferred over the use of FFP. vWF can be given as a blood derived concentrate in all VWD patients. Especially in type III VWD patients, who have a complete absence of VWF and DDAVP is of no use, and in type $2 b$ vWD patients, in whom unwanted clotting accompanied by a thrombocytopenia can occur when DDAVP is given, VWF can be lifesaving. Recombinant activated FVII (rFVIla) bypasses both the intrinsic and extrinsic pathway and directly activates $\mathrm{FX}$ in order to promote coagulation. Indications for $\mathrm{rFVIIa}$ are haemophilia A or B, FVII-deficiencies, and Glanzmann's thrombasthenia. In 
massive bleeding conditions, rFVIla has been used as a last resort, bypassing almost the entire coagulation cascade in order to stop the bleeding. Antifibrinolytics act by blocking the conversion of plasminogen to plasmin by tPA (tranexamic acid and $\varepsilon$-aminocaproic acid) or by inhibiting plasmin (aprotinin) thus preventing clot breakdown. Protamine as an antidote for unfractioned heparin is used mainly during cardiac and vascular surgery. Lastly, several steps in the coagulation cascade are calcium dependent. Calcium can be heavily depleted upon transfusion of multiple blood products, because of the citrate-based preservative solution in these blood products, and needs to be replenished accordingly. 


\section{Chapter 2}

\section{References}

1. Tanaka KA, Key NS, Levy JH. Blood coagulation: hemostasis and thrombin regulation. Anesth Analg. 2009 May;108(5):1433-46.

2. Linkins LA, Julian JA, Rischke J, Hirsh J, Weitz JI. In vitro comparison of the effect of heparin, enoxaparin and fondaparinux on tests of coagulation. Thromb Res. 2002 Sep 1;107(5):241-4.

3. Ganter MT, Hofer CK. Coagulation monitoring: current techniques and clinical use of viscoelastic point-of-care coagulation devices. Anesth Analg. 2008 May;106(5):1366-75.

4. MacDonald SG, Luddington RJ. Critical factors contributing to the thromboelastography trace. Semin Thromb Hemost. 2010 Oct;36(7):712-22.

5. Venema LF, Post WJ, Hendriks HG, Huet RC, de Wolf JT, de Vries AJ. An assessment of clinical interchangeability of TEG and ROTEM thromboelastographic variables in cardiac surgical patients. Anesth Analg. 2010 Aug;111(2):339-44.

6. Breet NJ, van Werkum JW, Bouman HJ, Kelder JC, Ruven HJ, Bal ET, Deneer VH, Harmsze AM, van der Heyden JA, Rensing BJ, Suttorp MJ, Hackeng CM, ten Berg JM. Comparison of platelet function tests in predicting clinical outcome in patients undergoing coronary stent implantation. JAMA. 2010 Feb 24;303(8):754-62.

7. Stissing T, Dridi NP, Ostrowski SR, Bochsen L, Johansson PI. The influence of low platelet count on whole blood aggregometry assessed by Multiplate. Clin Appl Thromb Hemost. 2011 Nov-Dec;17(6):E211-7.

8. Erber WN, Perry DJ. Plasma and plasma products in the treatment of massive haemorrhage. Best Pract Res Clin Haematol. 2006;19(1):97-112.

9. Brohi K, Cohen MJ, Davenport RA. Acute coagulopathy of trauma: mechanism, identification and effect. Curr Opin Crit Care. 2007 Dec;13(6):680-5.

10. Kozek-Langenecker SA. Effects of hydroxyethyl starch solutions on hemostasis. Anesthesiology. 2005 Sep;103(3):654-60.

11. Afshari A, Wikkelsø A, Brok J, Møller AM, Wetterslev J. Thrombelastography (TEG) or thromboelastometry (ROTEM) to monitor haemotherapy versus usual care in patients with massive transfusion. Cochrane Database Syst Rev. 2011 Mar 16;(3):CD007871.

12. Ten Cate H, Timmerman JJ, Levi M. The pathophysiology of disseminated intravascular coagulation. Thromb Haemost. 1999 Aug;82(2):713-7.

13. Toh CH, Hoots WK. The scoring system of the Scientific and Standardisation Committee on Disseminated Intravascular Coagulation of the International Society on Thrombosis and Haemostasis: a 5-year overview. J Thromb Haemost. 2007 Mar;5(3):604-6.

14. Kuiper GJ, Lancé MD, Smit-Fun VM, Peeters LL, Marcus MA. Platelet monitoring follow-up in a pregnant patient with HELLP syndrome. Platelets. 2011;22(2):160-3.

15. Lo GK, Juhl D, Warkentin TE, Sigouin CS, Eichler P, Greinacher A. Evaluation of pretest clinical score (4 T's) for the diagnosis of heparin-induced thrombocytopenia in two clinical settings. J Thromb Haemost. 2006 Apr;4(4):759-65.

16. Ten Cate H. Monitoring new oral anticoagulants, managing thrombosis, or both? Thromb Haemost. 2012 May;107(5):803-5. 
17. Tripodi A. The laboratory and the new oral anticoagulants. Clin Chem. 2013 Feb;59(2):353-62.

18. Levy JH and Tanaka KA. Prohemostatic agents to prevent perioperative blood loss. Semin Thromb Hemost. 2008 Jul;34(5):439-44.

19. Perkkiö J, Wurzinger LJ, Schmid-Schönbein H. Fåhraeus-Vejlens effect: margination of platelets and leukocytes in blood flow through branches. Thromb Res. 1988 May 1;50(3):357-64).

20. Kaufmann JE, Vischer UM. Cellular mechanisms of the hemostatic effects of desmopressin (DDAVP). J Thromb Haemost. 2003 Apr;1(4):682-9. 



\section{Chapter 3}

\section{The use of regression analysis} in determining reference intervals for low haematocrit and thrombocyte count in mul-

tiple electrode aggregometry

and platelet function analyzer

100 testing of platelet function

Kuiper GJ, Houben R, Wetzels RJ, Verhezen PW, van Oerle R, Henskens YM, Lancé MD

Platelets. 2017 Jan 9: 1-8. 


\section{Abstract}

Low platelet counts and haematocrit levels hinder whole blood point-of-care testing of platelet function. Thus far, no reference ranges for MEA (multiple electrode aggregometry) and PFA-100 (platelet function analyzer 100) devices exist for low ranges.

Through dilution methods of volunteer whole blood, platelet function at low ranges of platelet count and haematocrit levels was assessed on MEA for four agonists and for PFA-100 in two cartridges. Using (multiple) regression analysis, 95\% reference intervals were computed for these low ranges.

Low platelet counts affected MEA in a positive correlation (all agonists showed $r^{2} \geq 0.75$ ) and PFA100 in an inverse correlation (closure times were prolonged with lower platelet counts). Lowered haematocrit did not affect MEA testing, except for arachidonic acid activation (ASPI), which showed a weak positive correlation $\left(r^{2}=0.14\right)$. Closure time on PFA-100 testing was inversely correlated with haematocrit for both cartridges. Regression analysis revealed different $95 \%$ reference intervals in comparison with originally established intervals for both MEA and PFA-100 in low platelet or haematocrit conditions. Multiple regression analysis of ASPI and both tests on the PFA-100 for combined low platelet and haematocrit conditions revealed that only PFA-100 testing should be adjusted for both thrombocytopenia and anaemia. 95\% reference intervals were calculated using multiple regression analysis. However, coefficients of determination of PFA-100 were poor, and some variance remained unexplained.

Thus, in this pilot study using (multiple) regression analysis, we could establish reference intervals of platelet function in anaemia and thrombocytopenia conditions on PFA-100 and in thrombocytopenia conditions on MEA. 


\section{Chapter 3}

\section{Introduction}

Testing of platelet function comprises a variety of validated methods based on the use of platelet-rich plasma (PRP) or whole blood (point-of-care methods). Whole blood testing has the convenience of simple and fast operating procedures to complete a test and ease of interpreting obtained results. Multiple electrode aggregometry (MEA, Multiplate) is one of these tests in which whole blood is warmed and incubated for 3 minutes and after that tested for 6 more minutes. Multiple agonists can be used to start platelet activation and aggregation after the incubation phase. Platelet function is based on aggregation of platelets on two rods immerged in the incubated whole blood. This aggregation changes the impedance between these two rods, and this change in impedance is plotted as a graph. In the measuring cuvette, two pairs of rods are available, thus having an internal control with each measurement. PFA-100 (platelet function analyzer $100)$ testing is different in comparison with MEA and other methods. ${ }^{1}$ The PFA100 assesses platelet function while applying shear stress. Whole blood is added to a cuvette containing a small opening lined with collagen and either epinephrine or adenosine diphosphate (ADP); the blood flows under high shear stress (5000-6000 $\mathrm{s}^{-1}$ ) through the opening, which will close when sufficient platelets have aggregated; the time till occlusion (and thereby termination of blood flow) is defined as the closure time (CT).

One pitfall known in whole blood platelet function testing using the MEA or PFA100 method is that in order to obtain reliable results, a sufficient amount of platelets is needed. ${ }^{2-8}$ Platelet function testing is unreliable in low platelet ranges where thrombocyte transfusion is not yet required $\left(20-100 \times 10^{9} / \mathrm{L}\right.$ platelet range). ${ }^{2-8}$ Another pitfall is the presence of low haematocrit levels, especially in PFA-100 testing. ${ }^{9,10}$ A low haematocrit will prolong the closure time, and a haematocrit of at least $0.25 \mathrm{~L} / \mathrm{L}$ is needed for reliable results. ${ }^{9,11}$ However, for instance, during cardiothoracic surgery, when platelet function testing may be warranted in case of bleeding, often lower haematocrit levels are present (a 


\section{Chapter 3}

haemoglobin of $7 \mathrm{~g} / \mathrm{dL}$ being a global transfusion trigger of red cells, equals to roughly a haematocrit of $0.20-0.21 \mathrm{~L} / \mathrm{L}),{ }^{12}$ which hinders reliable platelet function testing. ${ }^{9,11}$

When using light transmission aggregometry (LTA), the current gold standard in platelet function testing using PRP, platelet levels are not to be adjusted before testing. ${ }^{13}$ In the past, Hayward and colleagues ${ }^{14}$ have established reference intervals for thrombocytopenic patients on LTA using regression analysis for various agonists. Thus by defining reference intervals, LTA became suitable for measuring platelet function in a wide range of platelet counts. And because LTA is a plasma-based analysis, haematocrit is irrelevant in this in vitro test.

For MEA and PFA-100 testing, research has been done in the past, hereby verifying that both these tests rely on adequate platelet counts and haematocrit levels. ${ }^{2,9}$ However, so far, no reference intervals have been established for either measuring device, which could guide users when encountering subjects with low platelet counts and/or haematocrit levels. Ideally, both devices would give the user reference intervals depending on these pre-analytical variables.

Our goal of this pilot study is to establish such reference intervals, similar to what has been done statistically for LTA, ${ }^{14}$ for each whole blood platelet function testing method by dilution of blood of healthy volunteers.

\section{Methods}

Blood sampling

Blood of healthy volunteers was used to obtain baseline and subsequent diluted whole blood samples for PFA-100 (Dade-Behring, Liederbach, Germany) and MEA (Dynabyte Medical, Munich, Germany) testing. Volunteers had to be at least 18 years of age. Volunteers were excluded if any medication, which could interfere with thrombocyte function, was used, or when they suffered from a bleeding diathesis. All blood donors volunteered and gave their consent for participation in this study. Twenty healthy volunteers were selected for each condition (PFA and low haematocrit, PFA and low platelets, MEA and low haematocrit, and MEA and low platelets). This was done because of budgetary reasons, time constraints, and manpower. Subsequently, a patient database 
search using all our MEA or PFA results was conducted in order to serve as positive control. Patients who had haematocrit levels below $0.25 \mathrm{~L} / \mathrm{L}$ and/or platelet counts below $150 \times 10^{9} / \mathrm{L}$ were selected. This study was carried out according to The Code of Ethics of the World Medical Association (Declaration of Helsinki). Our local institutional review board (azM/UM) has approved this study.

The basic principle was to use the volunteers' own blood to make dilutions of different haematocrit and thrombocyte levels, a technique similar to what Harrison et al performed in $1999 .{ }^{15}$ Blood was drawn via a clean venipuncture in 3-ml hirudin tubes (Verum Diagnostica, Munich, Germany) for MEA testing and in 4.5-ml 3.2\% (w/v) citrate tubes (Becton Dickinson BV, Breda, the Netherlands) for PFA-100 testing. A 5-mI EDTA tube (Becton Dickinson BV, Breda, the Netherlands) was collected first and used to calculate baseline haematocrit and thrombocyte counts on a Sysmex XE-5000 (Sysmex Nederland B.V., EttenLeur, The Netherlands).

\section{Blood preparation}

For testing the influence of thrombocyte counts, the following was done. One tube was tested without any centrifuging steps; four tubes were spun down for $10 \mathrm{~min}$ at $170 \mathrm{~g}$ to acquire platelet-rich plasma (PRP) on top of the buffy coat and red cell layer. Four more tubes were spun down for $5 \mathrm{~min}$ at 2,500 $\mathrm{g}$ to acquire platelet poor plasma (PPP) on top of the platelets, buffy coat, and red cell layer. A second centrifugation step was done at 10,000 $\mathrm{g}$ with the PPP for $10 \mathrm{~min}$ to acquire the final PPP solution. By substituting different amounts of PRP with PPP, thrombocyte counts were reduced while retaining original haematocrit levels. After substitution, tubes were resuspended to obtain "whole blood". We aimed for thrombocyte levels of $150,100,50$, and $20 \times 10^{9} / \mathrm{L}$ when diluting the samples. Of all samples, exact thrombocyte counts and haematocrits levels were measured. These were corrected for the amount of citrate (if applicable).

For determining the influence of haematocrit levels on platelet function, a similar but slightly different approach was used as follows: instead of substituting PRP 


\section{Chapter 3}

with PPP, this time equal volumes of the red cell layer were substituted with PPP, thus lowering the haematocrit levels while retaining thrombocyte levels. Haematocrit levels we aimed for were $0.35,0.30,0.25$, and $0.20 \mathrm{~L} / \mathrm{L}$ by diluting the samples. Again thrombocyte counts and haematocrit levels were measured and corrected for dilution by citrate in the citrate containing tubes.

\section{Platelet function analysis}

After these steps, closure time (CT) was analysed on PFA-100 using the epinephrine and ADP cartridge and we measured the area under the curve (AUC expressed in $U ; 1 \mathrm{U}$ equals $10 \mathrm{AU} \times \mathrm{x}$ min) on MEA using the TRAP (thrombin receptor activating peptide-6, final concentration (f.c.) $32 \mu \mathrm{mol} / \mathrm{L}$ ), COL (collagen, f.c. $3.2 \mu \mathrm{g} / \mathrm{mL}$ ), ADP (f.c. $6.5 \mu \mathrm{mol} / \mathrm{L}$ ), and ASPI (arachidonic acid, f.c. 0.5 $\mathrm{mmol} / \mathrm{L}$ ) reagents using standard protocols as provided by the manufacturer. Results were compared to previously in house defined upper and lower limit reference intervals as advised by the device manufacturers.

As a control, the effect of the centrifugation steps on thrombocyte function was assessed by first testing a whole blood sample on both machines using all cartridges and reagents, then centrifuging the sample, and then resuspending and testing the centrifuged sample once more.

\section{Statistics}

The results before and after centrifugation were compared using a paired Student's t-test. A $p$-value of $<0.05$ was considered to be significant. A (multiple) regression analysis was performed using (non-)linear curve fitting and in order to calculate coefficients of determination and predicted $95 \%$ reference intervals. A best fit was used in determining a linear or non-linear curve. Scatterplots of the (log transformed) standardized predicted values were used to see whether assumptions of homogeneity of variance and linearity were met. All statistics and regression analyses were done using SPSS Statistics v23 (IBM Corp, Armonk, NY, USA).

All results were plotted in a graph using GraphPad Prism (GraphPad Prism version 5.0a for Windows, GraphPad Software, San Diego, CA, USA). Using a 
(non-)linear fit, the 95\% prediction intervals were plotted as well using GraphPad Prism for each graph. Three-dimensional plots and contour plots were made online using WolframAlpha ${ }^{\circledR}$ (Wolfram Alpha LLC. 2009. Wolfram|Alpha (accessed on 17 March 2016). Available on http://www.wolframalpha.com).
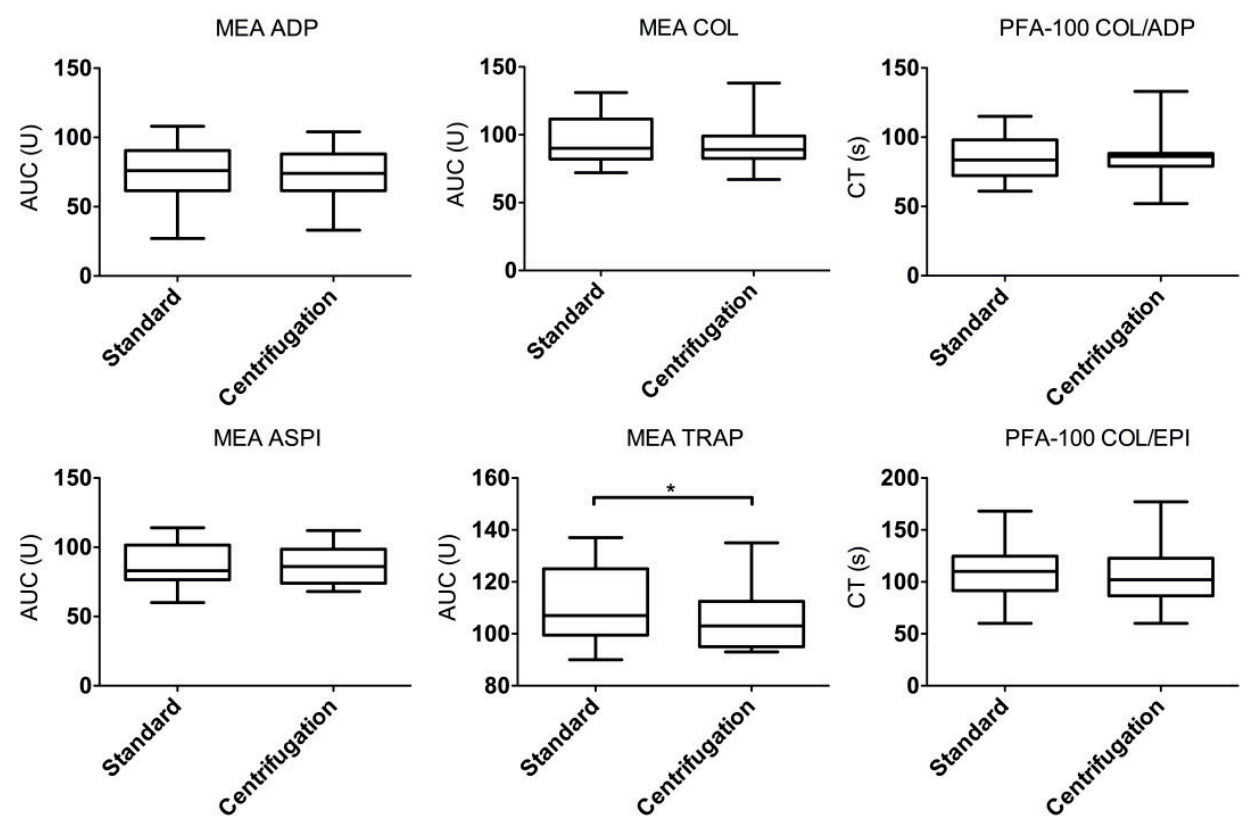

Figure 3.1 The effect of centrifugation on MEA and PFA-100 testing

The effect of centrifugation is shown here. Only in TRAP, a significant drop of 7.4 $\mathrm{U}$ was observed after centrifugation $(p=0.02)$. This was marked with an asterisk.

Abbreviations: ADP, adenosine diphosphate; ASPI, arachidonic acid; AUC, area under the curve; COL, collagen; CT, closure time; MEA, multiple electrode aggregometry; PFA-100 COL/ADP, platelet function analyzer 100 collagen/adenosine diphosphate cartridge; PFA-100 COL/EPI, platelet function analyzer 100 collagen/epinephrine cartridge; TRAP, thrombin receptor activating peptide-6; $\mathrm{U}, 10$ arbitrary units $\mathrm{x}$ min.

\section{Results}

\section{The effect of centrifugation on MEA and PFA-100 testing}

An alteration in platelet function due to centrifugation could not be found for $\operatorname{ADP}(p=0.30), \operatorname{COL}(p=0.13)$, and ASPI $(p=0.74)$ for MEA testing $(\mathrm{n}=20)$. For the TRAP reagent, there was a statistically significant difference $(p=0.02)$, with a mean lower AUC after centrifugation of $7.4 \mathrm{U}$ (figure 3.1). For the PFA100 , no alteration in platelet functionality due to centrifugation could be found for both cartridges (COL/EPI $p=0.29$, COL/ADP $p=0.57$ ). 


\section{Chapter 3}

Influence of platelet counts on MEA and PFA-100

Scatterplots of 20 subjects were made for each reagent separately with platelet counts on the $\mathrm{X}$-axis and either the AUC or CT on the $\mathrm{Y}$-axis (figure 3.2). The initial results without dilution are shown in blue squares. For each individual, four dilutions were made (squares in orange), bringing the total to 100 data points. In our patient database, 14 patients were found with platelet counts below $150 \times 10^{9} / \mathrm{L}$ for the MEA and 13 for the PFA-100. These are indicated in black dots.

For the TRAP $\left(r^{2}=0.75\right)$ and $\operatorname{COL}\left(r^{2}=0.76\right)$ reagents, best fit was acquired non-linear. For the ASPI $\left(r^{2}=0.80\right)$ and $\operatorname{ADP}\left(r^{2}=0.77\right)$ reagents, a linear fit was best. On the PFA-100, both reagents (COL/ADP $r^{2}=0.47$ and COL/EPI $r^{2}$ $=0.37$ ) were best fitted non-linearly. Standardized residual plots (after log transformation) showed that assumptions of normality, homoscedasticity, and linearity were met.

As can be observed from the MEA graphs, a positive correlation is apparent between platelet count and AUC, while an inverse correlation is present for the CT on the PFA-100.

Previously in house calculated 95\% reference ranges (grey areas), determined as advised by the manufacturer of each device, are also shown in the graphs. For PFA-100, platelet counts lower than approximately $90 \times 10^{9} / \mathrm{L}$ result in prolonged CT for both cartridges (the upper boundary of the manufacturer's recommended ranges is crossed), while for the MEA, the lower limit of platelet count is around $175 \times 10^{9} / \mathrm{L}$ for ADP, ASPI, and TRAP (here, the lower limit of the manufacturer's recommended ranges is crossed). For COL, the lower limit is around $50 \times 10^{9} / \mathrm{L}$.

For each of the reagents, a formula was calculated to compute the AUC for MEA testing or CT for PFA-100 analysis using regression analysis. Reference intervals (95\% prediction bands) were also computed and are given underneath each formula. 


$$
\begin{array}{ll}
\text { ADP AUC } & =-5.58+0.35 \times(\mathrm{PLT}) \\
& \pm 27.1 \text { for } 95 \% \text { prediction bands } \\
\text { ASPI AUC } & =3.84+0.41 \times(\mathrm{PLT}) \\
& \pm 29.8 \text { for } 95 \% \text { prediction bands } \\
\text { COL AUC } & =-114.3+92.6 \times \log (\mathrm{PLT}) \\
& \pm 31.7 \text { for } 95 \% \text { prediction bands } \\
\text { TRAP AUC } & =-130.9+100.0 \times \log (\mathrm{PLT}) \\
& \pm 36.0 \text { for } 95 \% \text { prediction bands } \\
\text { COL/ADP CT } & =396.4-139.4 \times \log (\mathrm{PLT}) \\
& \pm 60.8 \text { for } 95 \% \text { prediction bands } \\
\text { COL/EPI CT } & =471.0-161.8 \times \log (\mathrm{PLT}) \\
& \pm 85.2 \text { for } 95 \% \text { prediction bands }
\end{array}
$$

\section{Influence of haematocrit on MEA and PFA-100}

Again scatterplots of 20 subjects were made for each reagent separately with haematocrit on the $\mathrm{X}$-axis and either the AUC or CT on the $\mathrm{Y}$-axis (blue squares in figure 3.3). The diluted samples are shown in orange squares bringing the total once more to 100 . The black dots represent the patients from our database with a haematocrit below $0.25 \mathrm{~L} / \mathrm{L}$. We found three patients with PFA-100 results (one of these was not tested with the COL/EPI cartridge) and three with MEA results.

For the TRAP, ADP, and COL reagent, no fit could be made, as $r^{2}$ was $\leq 0.01$ in all cases. For the ASPI, a weak non-linear fit could be made with $r^{2}=0.14$. On the PFA-100, both reagents (COL/ADP $r^{2}=0.37$ and COL/EPI $r^{2}=0.31$ ) were best fitted non-linearly. Again, (log transformed) standardized residual plots showed that the assumptions of homogeneity of variance, normality, and linearity were met. 

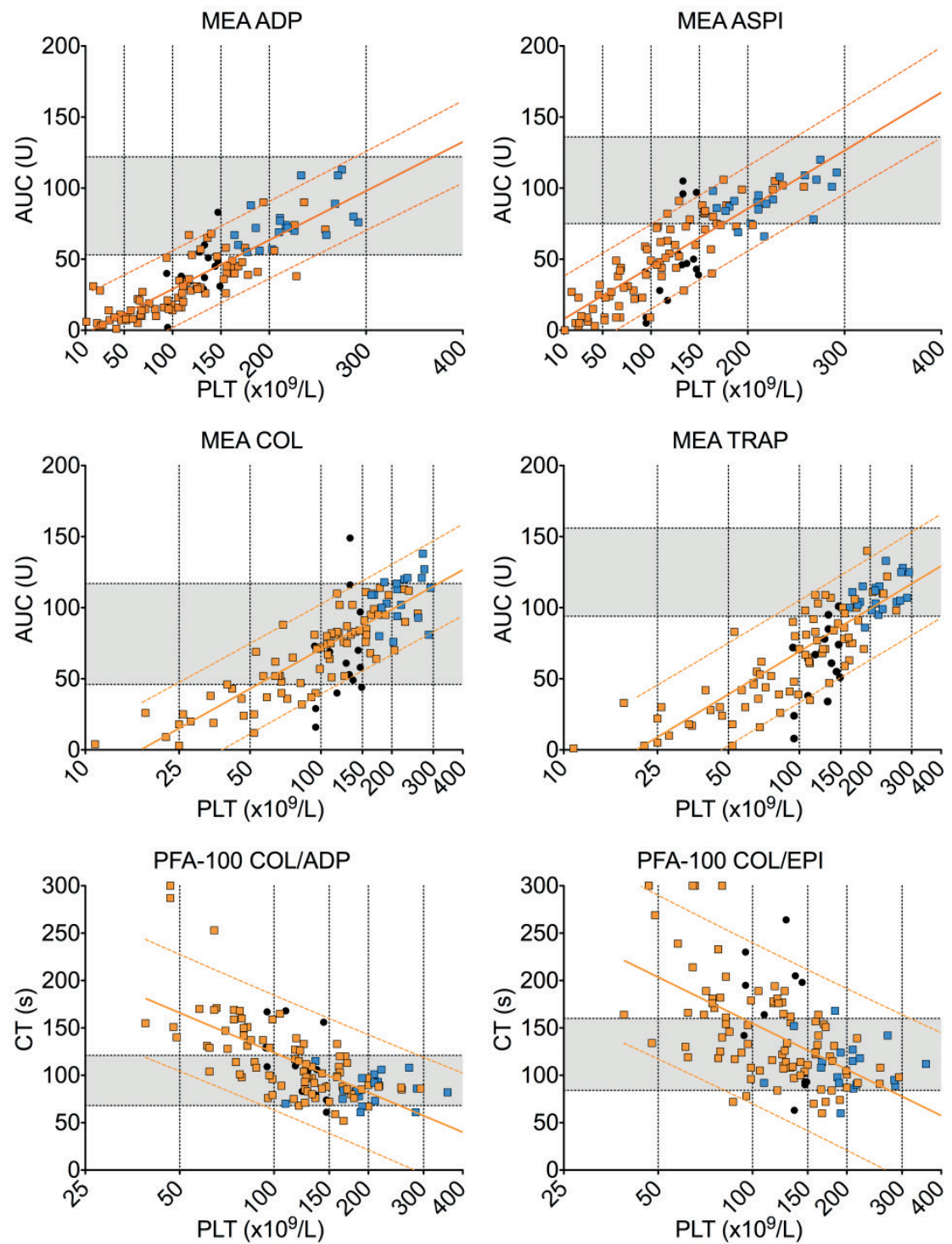

Figure 3.2 Influence of platelet count on MEA and PFA-100 testing. Blue squares are the original results of the healthy volunteers without dilution. The orange dots represent separate data points of the dilution steps. Black dots are patients from our MEA and PFA-100 database with platelet counts below $150 \times 10^{9} / \mathrm{L}$. Solid orange lines define the best fitting curve from regression analysis and the dotted orange lines its $95 \%$ reference intervals. Grey areas are according to the manufacturers advise in house defined upper and lower limits of normality.

Abbreviations: ADP, adenosine diphosphate; ASPI, arachidonic acid; AUC, area under the curve; COL, collagen; CT, closure time; L, liter; MEA, multiple electrode aggregometry; PFA-100 COL/ADP, platelet function analyzer 100 collagen/adenosine diphosphate cartridge; PFA-100 COL/EPI, platelet function analyzer 100 collagen/epinephrine cartridge; PLT, platelet count; TRAP, thrombin receptor activating peptide-6; $\mathrm{U}, 10$ arbitrary units $\mathrm{x}$ min. 

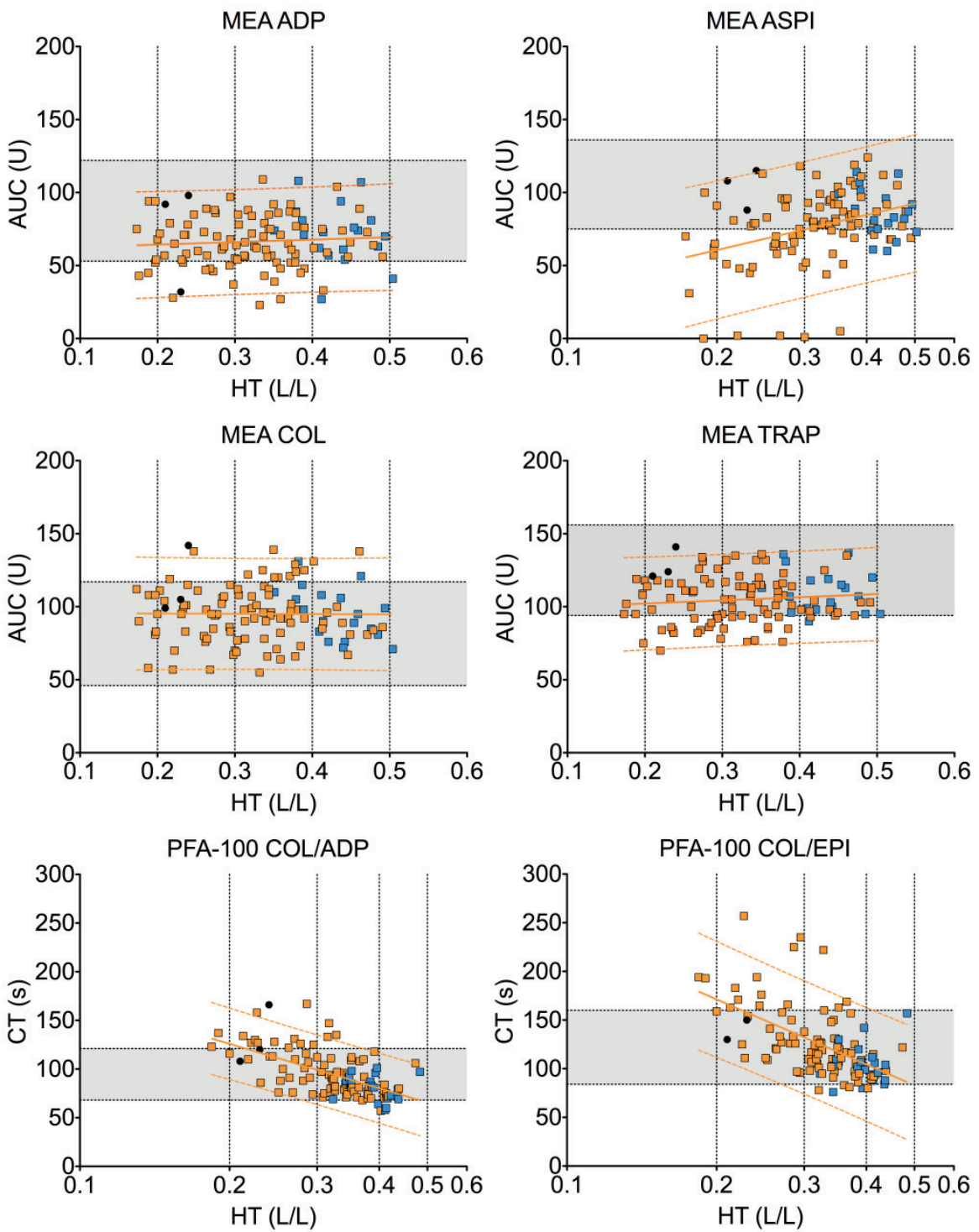

Figure 3.3 Influence of hematocrit levels on MEA and PFA-100 testing. Blue squares are the original results of the healthy volunteers without dilution. The orange dots represent separate data points of the dilution steps. Black dots are patients from our MEA and PFA-100 database with hematocrit below $0.25 \mathrm{~L} / \mathrm{L}$. Solid orange lines define the best fitting curve from regression analysis and the dotted orange lines its $95 \%$ reference intervals. Grey areas are according to the manufacturers advise in house defined upper and lower limits of normality.

Abbreviations: ADP, adenosine diphosphate; ASPI, arachidonic acid; AUC, area under the curve; COL, collagen; CT, closure time; HT, hematocrit; L, liter; MEA, multiple electrode aggregometry; PFA-100 COL/ADP, platelet function analyzer 100 collagen/adenosine diphosphate cartridge; PFA$100 \mathrm{COL/EPI}$, platelet function analyzer 100 collagen/epinephrine cartridge; TRAP, thrombin receptor activating peptide-6; $\mathrm{U}, 10$ arbitrary units $\mathrm{x}$ min. 


\section{Chapter 3}

As can be observed from the MEA graphs, a positive correlation is apparent between haematocrit and AUC for ASPI, while an inverse correlation is present once more for the CT on the PFA-100.

Previously in house calculated $95 \%$ reference ranges (grey areas), determined as advised by the manufacturer of each device, are also shown in the graphs. For PFA-100, haematocrit around $0.22 \mathrm{~L} / \mathrm{L}$ and below results in prolonged CT for both cartridges (by crossing the upper limit of the manufacturer's recommended ranges), while for the MEA, the lower limit of haematocrit is approximately $0.30 \mathrm{~L} / \mathrm{L}$ for ASPI (at this HT values drop below the manufacturer's recommended ranges).

Calculated formulas using regression analysis for each reagent are the following. Reference intervals (95\% prediction bands) are again computed and given underneath each formula.

$$
\begin{array}{ll}
\text { ADP AUC } & =65.0 \\
& \pm 35.9 \text { for } 95 \% \text { prediction bands } \\
\text { ASPI AUC } & =116.6+80.1 \times \log (\mathrm{HT}) \\
& \pm 46.6 \text { for } 95 \% \text { prediction bands } \\
\text { COL AUC } & =95.0 \\
& \pm 38.0 \text { for } 95 \% \text { prediction bands } \\
\text { TRAP AUC } & =105.0 \\
& \pm 31.4 \text { for } 95 \% \text { prediction bands } \\
\text { COL/ADP CT } & =19.4-151.8 \times \log (\mathrm{HT}) \\
& \pm 35.6 \text { for } 95 \% \text { prediction bands } \\
\text { COL/EPI CT } & =18.0-215.5 \times \log (\mathrm{HT}) \\
& \pm 58.2 \text { for } 95 \% \text { prediction bands }
\end{array}
$$


Multiple regression analysis of haematocrit and platelet count on MEA and PFA100

For interpretation of the AUC of the ASPI test and CT of both the PFA-100 tests, we corrected the results for platelet counts as well as haematocrit levels. This resulted in the following standard equation for MEA:

AUC $\quad=b 0+b 1 \times(P L T)+b 2 \times \log (H T)$

For the PFA-100 the following equation was used:

$$
\text { CT }=\mathrm{b} 0+\mathrm{b} 1 \times \log (\mathrm{PLT})+\mathrm{b} 2 \times \log (\mathrm{HT})
$$

Adjusted $r^{2}$ values of each model were 0.49 for ASPI, 0.30 for COL/ADP, and 0.25 for COL/EPI. This results in the following final equations for each of the three tests (reference intervals are below each formula):

$$
\begin{array}{ll}
\text { ASPI AUC } & =40.5+0.34 \times(\mathrm{PLT})+61.6 \times \log (\mathrm{HT}) \\
& \pm 43.5 \text { for } 95 \% \text { prediction bands } \\
\text { COL/ADP CT } & =210.7-80.1 \times \log (\mathrm{PLT})-134.2 \times \log (\mathrm{HT}) \\
& \pm 56.4 \text { for } 95 \% \text { prediction bands } \\
\text { COL/EPI CT } & =211.3-84.8 \times \log (\mathrm{PLT})-216.5 \times \log (\mathrm{HT}) \\
& \pm 78.0 \text { for } 95 \% \text { prediction bands }
\end{array}
$$

Three-dimensional plots and contour plots of the three equations above were made online using WolframAlpha ${ }^{\circledR}$ (figures $3.4,3.5$, and 3.6). On the $X$-axis, platelet count is shown, while on the $\mathrm{Y}$-axis, haematocrit levels are used. The resultant, AUC in U for ASPI testing on the MEA or CT in seconds for COL/ADP and COL/EPI on the PFA-100, can be observed with a colour gradient and the height on the Z-axis. These $3 \mathrm{D}$ and contour plots show that a combination of 


\section{Chapter 3}

a

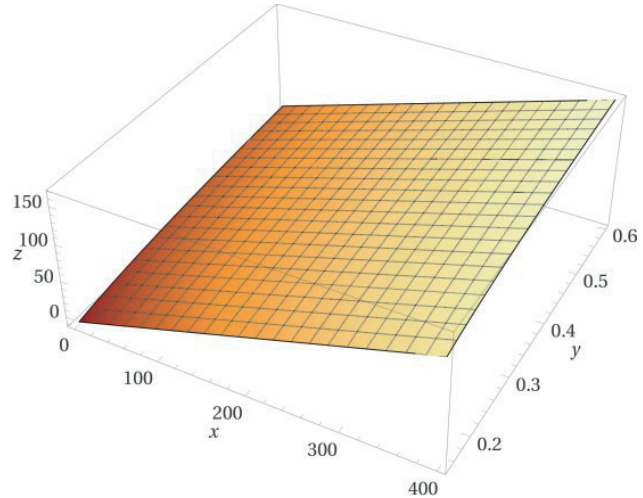

b

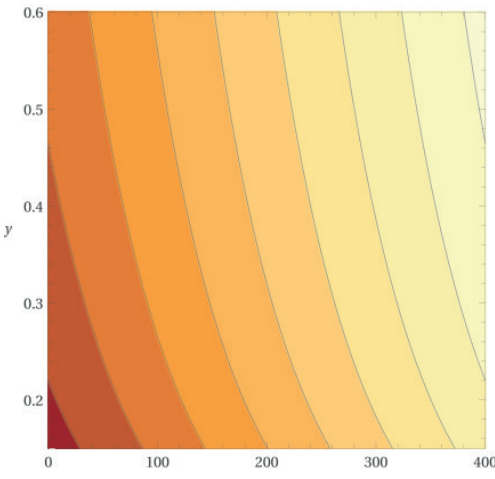

Figure 3.4 Influence of platelet count and haematocrit levels on MEA (ASPI) testing. 3D-graph for ASPI (4a) and top-down view (4b). The darker the colour, the lower the AUC on MEA. $x=$ PLT $\left(x 10^{9} / L\right), y=H T(L / L), z=A U C(U)$.

a

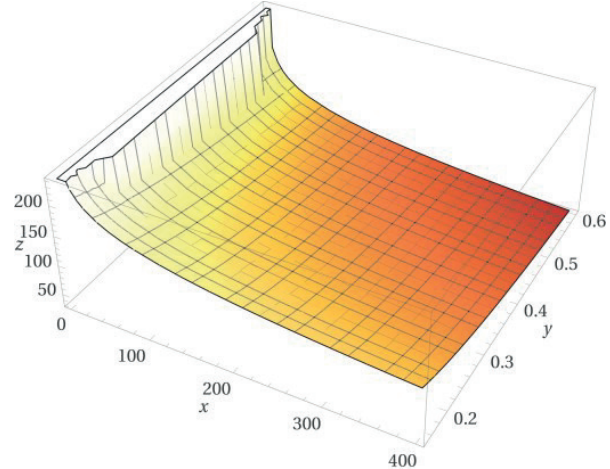

b

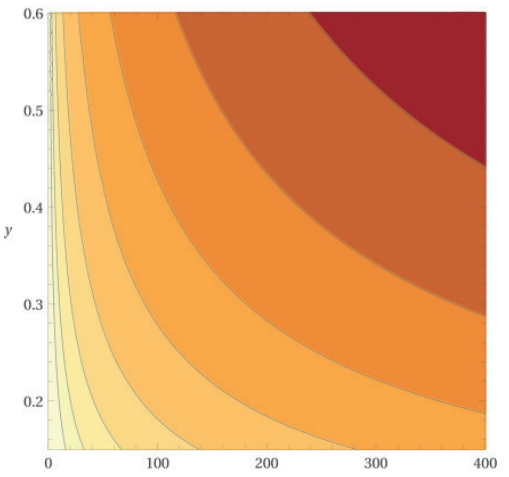

Figure 3.5 Influence of platelet count and haematocrit levels on PFA-100 (COL/ADP) testing. 3Dgraph for COL/ADP (5a) and top-down view (5b). The darker the colour, the shorter the CT on PFA100. $x=\operatorname{PLT}\left(x 10^{9} / L\right), y=H T(L / L), z=C T(s)$.

a

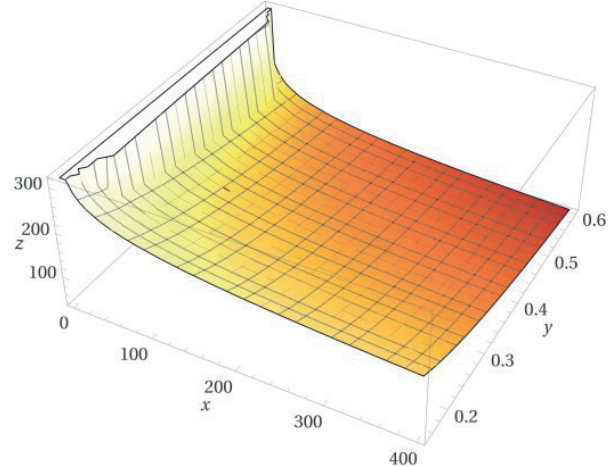

b

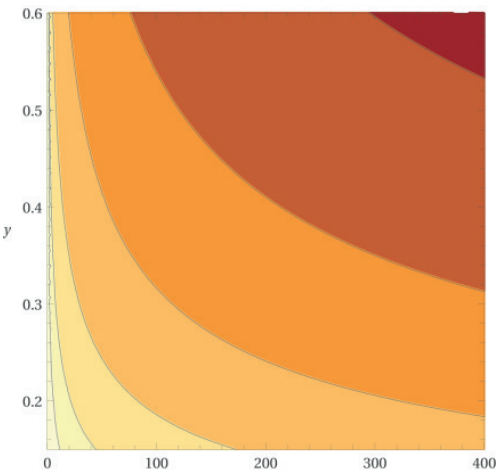

Figure 3.6 Influence of platelet count and haematocrit levels on PFA-100 (COL/EPI) testing. 3Dgraph for COL/EPI (6a) and top-down view (6b). The darker the colour, the shorter the CT on PFA100. $x=P L T\left(x 10^{9} / L\right), y=H T(L / L), z=C T(s)$. 
low platelet count together with low haematocrit results in either a low AUC for MEA (figure 3.4) or a prolonged CT for PFA-100 (figures 3.5 and 3.6). A high platelet count combined with high haematocrit results in either a high AUC for MEA (figure 3.4) or a shortened CT for PFA-100 (figures 3.5 and 3.6).

\section{Discussion}

In this pilot study, we defined reference ranges for platelet function testing on MEA and PFA-100 at low platelet counts and haematocrit levels using regression analysis and defined formulas for these reference intervals. In contrast to previous research, we tried to approach the clinical setting as much as possible, aiming for a condition of "pure" thrombocytopenia and anaemia, of variable degree, respectively. Stissing et al researched the effects of low platelet counts on MEA by diluting whole blood samples with PRP and PPP, hereby effectively also lowering haematocrit levels. ${ }^{5}$ Furthermore, they made grouping variables for the platelet counts and did not show standard deviations of their actual platelet counts for each group. Because of this, one cannot judge individual results of platelet counts in comparison with the AUC. Hanke and colleagues did similar experiments in MEA testing using platelet adjusted whole blood while retaining original haematocrit. Again they showed non-continuous data and used categories of low platelet count instead. ${ }^{8}$ Another research group also assessed the relation between platelet counts on MEA results by dilution with a crystalloid solution instead of autologous components. Their work, unfortunately, had to be retracted. ${ }^{16}$ What these previous authors evaluated for MEA, Harrison et al did for the PFA-100. ${ }^{15}$ Blood of healthy donors was used to assess the influence of thrombocytopenia and anaemia. They came to similar results as we did, but again could not give reference intervals. Besides that, they used blood of only three volunteers. Prolongation of CT by lowering platelet counts or haematocrit in vitro, shown by work of Kundu et al, ${ }^{11}$ was consistent to our study and an in vivo thrombocytopenia study by Carcao and colleagues proved the same relation between platelet count and the CT. ${ }^{17}$ Cho et al, however, stated that they could not find a relation between platelet counts and haematocrit and the CT in their reference interval finding study among healthy volunteers. ${ }^{8}$ 


\section{Chapter 3}

In our search for reference intervals, we first assessed if our method of lowering platelet counts and haematocrit levels by centrifugation and autologous blood mixing itself would affect our measurement on both devices. This could not be found except for the TRAP reagent on the MEA. This $7.4 \mathrm{U}$ diminishment due to centrifugation seems irrelevant when comparing this to the reference range of 94-156 U. Nevertheless, all our TRAP results should be interpreted as having a slightly false-low value when a sample was centrifuged.

We found that the AUC in MEA testing changes in a linear or a log-linear fashion (depending on the reagent) with lowering platelet counts. The coefficients of determination for all four tests were good as all showed an $r^{2} \geq 0.75$. We think that this non-linear relation could be explained by a ceiling effect of the agonist. TRAP as a strong activator of thrombocytes might induce a maximum activation of thrombocytes, hereby completely covering both rods in MEA. ADP is a much weaker activator of platelets, which might not be maximal in low to normal ranges, but eventually in high platelet ranges, it could be maxed out. Reference intervals, according to the manufacturer's recommendations, also point us in this direction. The upper limit of normality is lower in ADP (122 U) than it is for TRAP (156 U). For the PFA-100, thrombocyte counts have an inverse log-linear relationship with the $\mathrm{CT}$ for both cartridges. The coefficient of determination was acceptable for COL/ADP, but with an $r^{2}<0.40$, it was poor for COL/EPI. When looking at haematocrit levels, variation in haematocrit does not seem to be of relevance for the AUC in MEA testing, except for the ASPI test. Though the coefficient of determination was really low for ASPI, the CT on the PFA-100 fluctuates heavily with an inverse log-linear relation at different haematocrit levels.

The combined models all showed lower coefficients of determination, than when a single regression analysis was done. For MEA, this changed the $r^{2}$ from 0.80 for platelet counts and 0.14 for haematocrit level to 0.49 when combined. As a result, if measurements are to be done on the MEA, one should only correct them for platelet counts. Haematocrit does not seem to have a big influence on the AUC either alone or in conjunction with platelet count when testing platelet function on MEA. This is also apparent on the top-down view of the 3D graph 
for the ASPI (figure 3.4b): The isolines are hardly affected by differences in haematocrit level as they are plotted almost parallel to the haematocrit axis. When viewing the top-down views of both the 3D-graphs for the PFA-100 (figures $3.5 \mathrm{~b}$ and 3.6b), one can appreciate that haematocrit does influence the CT together with platelet count. Thus, for the PFA-100, one should adjust the results accordingly to these pre-analytical variables.

Using our MEA and PFA-100 database, we found several patients that met the criteria of low haematocrit and thrombocyte counts (black dots in figures 3.2 and 3.3). The AUC of most of the patients with low thrombocyte counts was below the grey standard reference ranges, but some of the results were within our newly defined reference intervals. This would mean a normal platelet function in these cases. On the other hand, some patients are below both reference areas. These patients, besides being thrombocytopenic, might have platelet defects due to the nature of their disease or due to antiplatelet drugs. This could not be resolved from the data we had in our database. Similar results were found for the PFA-100 in the thrombocytopenic patients. In the patients with anaemia (haematocrit below $0.25 \mathrm{~L} / \mathrm{L}$ ), this was not so evidently. This is probably due to the fact that only three patients could be selected based on their haematocrit.

For both multiple regression models, however, there is still a lot of variance to be explained. It is known from literature that results on the PFA-100 are dependent on von Willebrand factor (VWF). ${ }^{4,9}$ In a study by Van Vliet et al, ${ }^{19}$ type-1 von Willebrand disease (VWD) patients were treated with DDAVP (desmopressin) or Haemate-P (a factor VIII/VWF concentrate) and the effects were measured with the PFA-100 COL/EPI cartridge. This study showed a marked effect of changes in VWF which were assessed by relatively high coefficients of determination for VWF:CB (VWF:collagen binding), VWF:Ag (vWF:antigen), VWF:RCo (VWF:ristocetin cofactor), and factor VIII (all above 0.5 except for VWF:Ag in the Haemate-P pre- and post-treatment group $\left.\left(r^{2}=0.42\right)\right)$. Ideally, we would like to have measured vWF activity or antigen in our diluted samples to see its effect. This effect of vWF could explain why in PFA-100 testing we have a non-linear regression. Due to a synergistic effect of vWF adhesion to 


\section{Chapter 3}

platelets and vice versa, they enhance each other in an exponential fashion. Unfortunately, we do not have plasma remaining and correction of the PFA-100 for vWF activity or antigen would nullify the use of the PFA-100 as a screening tool for VWD.

One drawback of this study is that because we lowered the platelet count and the haematocrit level separately, we did not mimic the situation of a combined thrombocytopenia and anaemia condition. Therefor contrast is lost in this combined thrombocytopenia and anaemia range. However, it is very unlikely that a low platelet count in combination with a low haematocrit level would promote improved platelet function.

In this pilot study, we have shown that MEA testing of platelet function is dependent on platelet count and that PFA-100 testing of platelet function is dependent on both platelet count as well as haematocrit levels. For both testing methods, we were able to define variable reference intervals using (multiple) regression analysis and proposed formulas to guide users in interpreting MEA and PFA-100 results in case of thrombocytopenic and anaemic patients. These data, however, need further validation in a new set of healthy volunteers and/or in more patients with anaemia or thrombocytopenia. 


\section{References}

1. Berkowitz SD, Frelinger AL 3rd, Hillman RS. Progress in point-of-care laboratory testing for assessing platelet function. Am Heart J. 1998 Oct;136(4 Pt 2 Su):S51-65.

2. Bochsen L, Johansson PI, Kristensen AT, Daugaard G, Ostrowski SR. The influence of platelets, plasma and red blood cells on functional haemostatic assays. Blood Coagul Fibrinolysis. 2011 Apr;22(3):167-75.

3. Rubak P, Villadsen K, Hvas AM. Reference intervals for platelet aggregation assessed by multiple electrode platelet aggregometry. Thromb Res. 2012 Sep;130(3):420-3.

4. Seyfert UT, Haubelt H, Vogt A, Hellstern P. Variables influencing Multiplate(TM) whole blood impedance platelet aggregometry and turbidimetric platelet aggregation in healthy individuals. Platelets. 2007 May;18(3):199-206.

5. Stissing T, Dridi NP, Ostrowski SR, Bochsen L, Johansson PI. The influence of low platelet count on whole blood aggregometry assessed by Multiplate. Clin Appl Thromb Hemost. 2011 Nov-Dec;17(6):E211-7.

6. Tóth O, Calatzis A, Penz S, Losonczy H, Siess W. Multiple electrode aggregometry: a new device to measure platelet aggregation in whole blood. Thromb Haemost. 2006 Dec;96(6):7818.

7. Würtz M, Hvas AM, Kristensen SD, Grove EL. Platelet aggregation is dependent on platelet count in patients with coronary artery disease. Thromb Res. 2012 Jan;129(1):56-61.

8. Hanke AA, Roberg K, Monaca E, Sellmann T, Weber CF, Rahe-Meyer N, Görlinger K. Impact of platelet count on results obtained from multiple electrode platelet aggregometry (Multiplate). Eur J Med Res. 2010 May 18;15(5):214-9.

9. Favaloro EJ. Clinical application of the PFA-100. Curr Opin Hematol. 2002 Sep;9(5):407-15.

10. Hayward CP, Harrison P, Cattaneo M, Ortel TL, Rao AK. Platelet function analyzer (PFA)-100 closure time in the evaluation of platelet disorders and platelet function. J Thromb Haemost. 2006 Feb;4(2):312-9.

11. Kundu SK, Heilmann EJ, Sio R, Garcia C, Davidson RM, Ostgaard RA. Characterization of an In Vitro Platelet Function Analyzer, PFA-100'TM. Clin Appl Thromb Hemost. 1996 Oct;2(4):241249.

12. Likosky DS, FitzGerald DC, Groom RC, Jones DK, Baker RA, Shann KG, Mazer CD, Spiess $\mathrm{BD}$, Body SC. The effect of the perioperative blood transfusion and blood conservation in cardiac surgery Clinical Practice Guidelines of the Society of Thoracic Surgeons and the Society of Cardiovascular Anesthesiologists upon clinical practices. J Extra Corpor Technol. 2010 Jun;42(2):114-21.

13. Cattaneo M, Cerletti C, Harrison P, Hayward CP, Kenny D, Nugent D, Nurden P, Rao AK, Schmaier AH, Watson SP, Lussana F, Pugliano MT, Michelson AD. Recommendations for the Standardization of Light Transmission Aggregometry: A Consensus of the Working Party from the Platelet Physiology Subcommittee of SSC/ISTH. J Thromb Haemost. 2013 Apr;11(6):11839. 


\section{Chapter 3}

14. Hayward CP, Moffat KA, Pai M, Liu Y, Seecharan J, McKay H, Webert KE, Cook RJ, Heddle NM. An evaluation of methods for determining reference intervals for light transmission platelet aggregation tests on samples with normal or reduced platelet counts. Thromb Haemost. 2008 Jul;100(1):134-45.

15. Harrison P, Robinson MS, Mackie IJ, Joseph J, McDonald SJ, Liesner R, Savidge GF, Pasi J, Machin SJ. Performance of the platelet function analyser PFA-100 in testing abnormalities of primary haemostasis. Blood Coagul Fibrinolysis. 1999 Jan;10(1):25-31.

16. Retraction: Whole-blood aggregometry: are there any limits with regard to platelet counts? Acta Anaesthesiol Scand. 2011 Aug;55(7):903.

17. Carcao MD, Blanchette VS, Stephens D, He L, Wakefield CD, Butchart S, Christie DJ, Rand ML. Assessment of thrombocytopenic disorders using the Platelet Function Analyzer (PFA-100). Br J Haematol. 2002 Jun;117(4):961-4.

18. Cho YU, Jang S, Park CJ, Chi HS. Variables that affect platelet function analyzer-100 (PFA100) closure times and establishment of reference intervals in Korean adults. Ann Clin Lab Sci. 2008 Summer;38(3):247-53.

19. Van Vliet HH, Kappers-Klunne MC, Leebeek FW, Michiels JJ. PFA-100 monitoring of von Willebrand factor (VWF) responses to desmopressin (DDAVP) and factor VIIINWF concentrate substitution in von Willebrand disease type 1 and 2. Thromb Haemost. 2008 Sep;100(3):462-8. 


\section{Chapter 4}

\section{Routine haemostasis testing} before transplanted kidney

\section{biopsy: a cohort study}

Kuiper GJ, Christiaans MH, Mullens HJ, ten Cate H, Hamulýak K, Henskens YM

Transpl Int. 2017 Nov 6. 


\section{Abstract}

\section{Background}

Kidney biopsy can result in bleeding complications. Pre-biopsy testing using bleeding time (BT) is controversial. New whole blood haemostasis tests, like platelet function analyser 100 (PFA-100) and multiple electrode aggregometry (MEA) might perform better. We postulated that PFA-100 would be suitable to replace BT pre-biopsy.

\section{Methods}

In 154 patients, transplanted kidney biopsies were performed after measurement of bleeding time, PFA-100, MEA, and mean platelet volume (MPV). Bleeding outcome (haemoglobin $(\mathrm{Hb})$ drop, haematuria ( \pm bladder catherization), ultrasound finding of a bleeding, need for (non-)surgical intervention, and/or transfusion) after the biopsy was correlated to each test.

\section{Results}

Male-female ratio was 2:1. 50\% had a surveillance biopsy at either three or 12 months. Around $17 \%$ (had) used acetylsalicylic acid (ASA) pre-biopsy. Of 17 bleeding events one subject needed a transfusion. Most bleeding events were $\mathrm{Hb}$ reductions over $1 \mathrm{mmol} / \mathrm{L}$ and all resolved uneventful. BT, PFA-100, MEA, and MPV did not predict a bleeding outcome; prior ASA use however could (odds ratio $3.19 ; 95 \%-\mathrm{Cl} 1.06$ to 9.61). Diagnostic performance data and Bland-Altman analysis showed that BT could not be substituted by PFA-100.

\section{Conclusions}

ASA use was the best determinant of bleeding after kidney biopsy. Routine haemostasis testing pre-biopsy has no added value. 


\section{Chapter 4}

\section{Introduction}

In up to $90 \%$ of all kidney biopsies the procedure is uneventful. Incidences of major bleeding complications requiring surgical intervention or resulting in death are low $(<0.5 \%)$, making it a safe diagnostic procedure. ${ }^{1}$ Biopsies of transplanted kidneys have a lower overall complication risk (6\% has only minor complications) compared to native kidneys, a finding that so far remains unexplained. ${ }^{2}$ In a large registry of over 8,500 biopsies, macroscopic haematuria was seen most frequently, in around $2 \%$ of all native kidney biopsies, while the need for transfusion was seen in less than $1 \%{ }^{3}$ Risk factors contributing to these bleeding complications are well described..$^{3-9}$ In spite of the apparent overall safety, many nephrologists are reluctant to proceed to biopsy without any laboratory testing. At the same time controversy remains about the added value of laboratory testing and in particular about the utility of the bleeding time, ${ }^{10-14}$ a rather poor performing test that remains persistently in use, despite its accepted shortcomings. From a traditional perspective, it is still often routine practice ${ }^{15}$ to assess a bleeding time prior to kidney biopsy as an overall haemostasis test. The common rationale is that in chronic kidney disease uremic patients have an acquired platelet dysfunction as well as deranged coagulation system. ${ }^{12,16}$ Prolonged bleeding times trigger physicians to administer 1-desamino-8-D-arginine vasopressin (DDAVP) in order to enhance platelet function during kidney biop$\mathrm{sy} ;{ }^{1,17}$ it has been proven to reduce haematoma formation after this procedure. ${ }^{1,16,18}$ In the Netherlands consensus on the use of specific laboratory tests is lacking, in spite of a previous study from this country comparing a historical cohort using the bleeding time with a prospective cohort of the platelet function analyzer 100 (PFA-100). ${ }^{19}$

Nowadays an array of in vitro platelet function tests is available on the market that could at least supersede the in vivo bleeding time. Multiple electrode aggregometry (MEA, Multiplate) is an in vitro whole blood point-of-care test device of platelet function in which platelet activators (i.e. COL (collagen), ADP (aden- 


\section{Chapter 4}

osine diphosphate), ASPI (arachidonic acid), or TRAP (thrombin receptor activating peptide-6)) are added and platelet aggregation is measured using an impedance method. PFA-100 also measures activated platelets but it incorporates flow, making it more sensitive to the effects of von Willebrand factor. Both tests depend on platelet count, while PFA-100 results need to be corrected for haematocrit as well. ${ }^{20}$ PFA-100 is more sensitive in comparison to the bleeding time for bleeding disorders, however could still produce false-negatives in mild bleeding disorders, ${ }^{21,22}$ for which MEA might perform better. Additionally, mean platelet volume (MPV) is an indirect measure of platelet turnover and is related to platelet reactivity. ${ }^{23} \mathrm{~A}$ high MPV is seen in states of high megakaryocyte stimulation due to thrombocytopenia. ${ }^{24}$ MPV could be helpful in predicting bleeding.

Therefore, in this study we have investigated which of these tests (Ivy bleeding time, MEA, PFA-100, or MPV), if any, could predict bleeding after kidney biopsy, assuming that the PFA-100 would be superior to the bleeding time, due to its potential higher sensitivity. ${ }^{22}$

\section{Materials and methods}

\section{Study design and in- and exclusion criteria}

We organized a prospective observational study in chronic kidney disease patients, who were scheduled to undergo a biopsy of a previously retroperitoneal transplanted kidney in the iliac fossa. Subjects were included at three or 12 months check-up for surveillance kidney biopsy, or outside these scheduled check-ups for other various reasons (non-surveillance biopsies). Minors (age $<18$ years) were excluded from this study. Other exclusion criteria were known hereditary bleeding disorders, platelet count $<80 \times 10^{9} / \mathrm{L}$, or haematocrit $<0.25$ L/L. Subjects on antithrombotic medication use were managed as follows. Acetylsalicylic acid (ASA) was stopped at least five days pre-biopsy. All low molecular weight heparins (LMWH) were stopped and anti-Xa testing was done to rule out residual heparin activity before biopsy. The use of vitamin $\mathrm{K}$ antagonists was stopped and INR had to be $<1.6$ pre-biopsy. All subjects gave informed consent prior to enrolment in this study for extra blood sampling and analysis. 
This study was carried out according to The Code of Ethics of the World Medical Association (Declaration of Helsinki). Our local institutional review board approved the conductance of this study.

\section{Routine tests and reference intervals}

According to routine care the following tests were done prior to the biopsy: haemoglobin level $(\mathrm{Hb})$, thrombocyte count (PLT), and mean platelet volume (MPV) in ethylenediaminetetraacetic acid (EDTA) anticoagulated blood (collected in $5 \mathrm{~mL}$ K2-EDTA tubes, Becton Dickinson BV, Breda, The Netherlands) on a Sysmex XE-5000 machine (Sysmex Nederland B.V., Etten-Leur, The Netherlands). Anaemia was defined as an $\mathrm{Hb}$ below $8.2 \mathrm{mmol} / \mathrm{L}$ for men and below 7.3 $\mathrm{mmol} / \mathrm{L}$ for women. Thrombocytopenia was defined by a PLT lower than 130 $x 10^{9} / \mathrm{L}$. For MPV, results were interpreted according to our previously established reference intervals of 9.2 to $12.7 \mathrm{fL} .{ }^{23}$ The activated partial thromboplastin time (aPTT) and prothrombin time corrected by the international normalized ratio (PT-INR) were determined in citrate anticoagulated plasma (collected in $4.5 \mathrm{~mL} 3.2 \%(\mathrm{w} / \mathrm{v})$ citrate tubes, Becton Dickinson BV, Breda, The Netherlands). An aPTT over 32 seconds and a PT-INR higher than 1.2 were both considered abnormal. Urea and creatinine levels were measured in serum plasma tubes (Becton Dickinson BV, Breda, The Netherlands). Estimated glomerular filtration rate (eGFR) was calculated according to the MDRD-formula. ${ }^{25}$ Subjects were grouped by disease state according to the kidney disease outcomes quality initiative (K/DOQI) guidelines. ${ }^{26}$ Elevated urea was defined as a urea greater than the upper limit of normality (>8 $\mathrm{mmol} / \mathrm{L}$ ). High urea was defined as a urea greater than twice the upper limit of normality (>16 mmol/L).

Index tests and reference intervals

Bleeding time was performed according to the Ivy method. ${ }^{27}$ Three consecutive cuts were made by a trained technician and the average bleeding time was noted. Bleeding times over 4 minutes were considered to be abnormal.

Multiplate was performed in hirudin anticoagulated whole blood according to the manufacturer's guidelines. Platelet aggregation was initiated by adding one of 


\section{Chapter 4}

the following reagents: ADP (final concentration, f.c. $6.5 \mu \mathrm{M}$ ), ASPI (f.c. 0.5 $\mathrm{mM}$ ), $\mathrm{COL}$ (f.c. $3.2 \mu \mathrm{g} / \mathrm{ml}$ ), and TRAP (f.c. $32 \mu \mathrm{M}$ ). Area under the curve (AUC in $\mathrm{U} ; 1 \mathrm{U}$ equals 10 Arbitrary Units $\mathrm{x} \mathrm{min}$ )) of the Multiplate results was calculated. AUC was corrected for thrombocyte count according to Kuiper et $\mathrm{al}^{20}$ in order to determine whether an AUC was within normal range or reduced.

PFA-100 testing was done in citrate anticoagulated whole blood using both the COL/ADP as well as the COL/EPI cartridge according the manufacturer's instructions. Closure time (CT) in seconds was used for analysis. CT was corrected for thrombocyte count and haematocrit level according to Kuiper et $\mathrm{al}^{20}$ in order to determine whether a CT was within normal range or prolonged.

\section{Procedure}

Although DDAVP could be given at $0.3 \mu \mathrm{g} / \mathrm{kg}$ iv prior to the biopsy, if deemed necessary, none of the subjects actually received DDAVP during this study. For all biopsies a 16-gauge ultrasound guided spring-loaded biopsy gun was used, after administration of local anaesthetics at the intended puncture site. Biopsies were performed by radiologists experienced in doing so or radiologists in training under supervision of an experienced radiologist. A minimum of three samples were taken and investigated directly after biopsy for adequacy with the aid of a magnifying glass for the presence of glomeruli. Following biopsy all subjects were kept bedridden for six hours with a one $\mathrm{kg}$ bag of sand resting on the puncture site. Subjects were monitored with a follow up of the puncture site for the first two hours and a urine analysis with complete blood count at four hours post biopsy. After the six hours bed rest, subjects were allowed to mobilize and were discharged if all checks were normal. A control ultrasound was performed within 24 hours after biopsy in case of clinical suspicion of a bleeding complication ( $\mathrm{Hb}$ drop, pain, macroscopic haematuria, and/or hypotension).

\section{Endpoint definitions}

A haemoglobin drop of one $\mathrm{mmol} / \mathrm{L}$ or more (equal to $1.61 \mathrm{~g} / \mathrm{dL}$ ) was considered to have clinical consequences and thus be a bleeding outcome. A positive ultrasound finding of a perinephric and/or subcapsular haematoma after biopsy 
was also deemed a bleeding endpoint. Haematuria detected with microscopic urine analysis (defined in our study as $>200$ red blood cells per high power field (RBC/HPF)), which was not present pre-biopsy and lasted longer than 24 hours was also defined as a bleeding outcome. Only subjects needing a post-biopsy (surgical) intervention (e.g. embolization, coiling, explantation, transfusion of packed cells, bladder catheterization, or other) were marked having a major bleeding complication. All bleeding complications without need for an intervention were considered to be non-major (i.e. minor) bleeding endpoints.

\section{Statistical analysis}

All the statistic testing was performed using SPSS v23 (IBM Corp, Armonk NY USA). Categorical and ordinal data are expressed as number with percentage. Interval data is expressed as median with interquartile range (IQR). One-Way ANOVA with post-hoc correction for multiple comparisons was performed for the occurrence of differences between variables among the different chronic kidney disease (CKD) stadia. For equal variances a Hochberg's GT2 and for non-equal variance a Games-Howell correction was used. Odds ratios (OR) of binominal data were calculated for relevant (risk) factors. PFA-100 and MEA results of all subjects were plotted in GraphPad Prism (GraphPad Prism version 5.0a for Windows, GraphPad Software, San Diego California USA) with the previously researched reference intervals. ${ }^{20}$ Diagnostic performance data (sensitivity, specificity, area under the receiver operating characteristic curve (ROCAUC), positive predictive value (PPV), negative predictive value (NPV), positive likelihood ratio (PLHR), and negative likelihood ratio (NLHR) for each test and an endpoint; kappa between BT and each test in correctly predicting a bleeding endpoint) was computed using the pooled data after a multiple imputation procedure $(\mathrm{N}=5)$ because of data missing at random. In order to see if the bleeding time could be substituted by PFA-100, Bland-Altman analysis reporting bias and 95\% limits of agreement was performed.

For all analyses a p-value below 0.05 was considered statistically significant. 


\section{Chapter 4}

\section{Results}

\section{Baseline characteristics}

A total of 154 subjects were included. $64 \%$ were of male gender and the overall median age was 59 years (interquartile range (IQR) was 48.8 to 66). Around half of the subjects had a surveillance biopsy at either three or 12 months posttransplantation. ASA use was reported in 26 subjects and stopped adequately

Table 4.1 Baseline patient, clinical, and test characteristics

\begin{tabular}{|c|c|}
\hline Baseline Demographics & All biopsies $(n=154)$ \\
\hline \multicolumn{2}{|l|}{ Patient characteristics } \\
\hline Male gender - no. (\%) & $98(63.6)$ \\
\hline Age - years (IQR) & $59.0(48.8-66)$ \\
\hline Surveillance post-transplant biopsy - no. (\%) & $83(53.9)$ \\
\hline After 3 months - no. (\%) & $48(31.2)$ \\
\hline After 12 months - no. (\%) & $35(22.7)$ \\
\hline Had previous biopsy in study - no. (\%) & $21(13.6)$ \\
\hline Acetylsalicylic acid use - no. (\%) & $26(16.9)$ \\
\hline \multicolumn{2}{|l|}{ Clinical characteristics } \\
\hline Systolic blood pressure - mmHg (IQR) & $140(127-150)$ \\
\hline Diastolic blood pressure - mmHg (IQR) & $77(70-85)$ \\
\hline Haemoglobin - mmol/L (IQR) & $7.0(6.2-8.3)$ \\
\hline Anaemia (male: $<8.2$; female: $<7.3 \mathrm{mmol} / \mathrm{L})$ - no. $(\%)$ & $97(63.0)$ \\
\hline Haematocrit - L/L (IQR) & $0.34(0.30-0.40)$ \\
\hline Thrombocyte count - x10 $1 / \mathrm{L}(\mathrm{IQR})$ & $222(177-284)$ \\
\hline Thrombocytopenia (<130 x10\%/L) - no. (\%) & $9(5.8)$ \\
\hline Mean platelet volume - $\mathrm{fL}(\mathrm{IQR})^{*}$ & $8.2(7.5-9.0)$ \\
\hline Low $(<9.2 \mathrm{fL})-$ no. $(\%)$ & $106(79.1)$ \\
\hline Normal - no. (\%) & $27(20.1)$ \\
\hline High (>12.7 fL) - no. (\%) & $1(0.7)$ \\
\hline PT-INR - s/s (IQR) & $1.0(1.0-1.1)$ \\
\hline PT-INR normal - no. (\%) & $145(96.7)$ \\
\hline PT-INR prolonged (>1.2) - no. (\%) & $5(3.3)$ \\
\hline aPTT - s (IQR) & $28(27-30)$ \\
\hline aPTT normal - no. (\%) & $129(86.6)$ \\
\hline aPTT prolonged (>32 s) - no. (\%) & $20(13.4)$ \\
\hline Urea - mmol/L (IQR) & $11.1(7.8-17.4)$ \\
\hline Normal - no. (\%) & $41(27.0)$ \\
\hline Elevated (>8 to $16 \mathrm{mmol} / \mathrm{L})-$ no. $(\%)$ & $67(44.1)$ \\
\hline High (>16 mmol/L) - no. (\%) & $44(28.9)$ \\
\hline Creatinine $-\mu \mathrm{mol} / \mathrm{L}(\mathrm{IQR})^{\star \star \star *}$ & $212(132-434)$ \\
\hline MDRD-eGFR - mL/min/1.73m² (IQR) ${ }^{* * * *}$ & $29.0(12.3-46.0)$ \\
\hline CKD Stage I-II - no. (\%) & $13(8.4)$ \\
\hline CKD Stage III - no. (\%) & $61(39.6)$ \\
\hline CKD Stage IV - no. (\%) & $32(20.8)$ \\
\hline CKD Stage V - no. (\%) & $46(29.9)$ \\
\hline \multicolumn{2}{|l|}{ Test characteristics } \\
\hline Performed Ivy bleeding time - no. (\%) & $150(97.4)$ \\
\hline Performed PFA-100 - no. (\%) & $151(98.1)$ \\
\hline Performed MEA - no. (\%) & $148(96.1)$ \\
\hline
\end{tabular}

Measured in amount of biopsies: ${ }^{*} n=134 ;{ }^{* *} n=150 ;{ }^{* * *} n=149 ;{ }^{* * * *} n=152$

Abbreviations: aPTT, activated partial thromboplastin time; CKD, chronic kidney disease; IQR, interquartile range; MEA, multiple electrode aggregometry; PFA-100, Platelet Function Analyser 100; PT-INR, prothrombin time correct for international normalized ratio 
five days pre-biopsy in 25 of the subjects. One subject had a protocol violation by not having stopped its use five days pre-biopsy. Anaemia was present in $63 \%$ of all subjects, while $5.8 \%$ had a thrombocytopenia (thrombocyte counts $\left.<130 \times 10^{9} / \mathrm{L}\right)$. MPV data were available in 134 of all studied subjects, which revealed an abnormally low MPV in around $79 \%$ of all subjects. Other baseline characteristics can be found in table 4.1.

\section{Endpoint characteristics}

17 subjects $(11 \%)$ had a clinically relevant bleeding endpoint (table 4.2 ). In three subjects a positive ultrasound for haematoma formation was found. Two of these subjects had a non-relevant drop in haemoglobin $(<1 \mathrm{mmol} / \mathrm{L})$, while the other subject had an accompanying haemoglobin drop of $1.8 \mathrm{mmol} / \mathrm{L}$ and needed transfusion of packed cells at a haemoglobin of $3.9 \mathrm{mmol} / \mathrm{L}$; otherwise, the patient recovered uneventful. 13 subjects with a relevant $\mathrm{Hb}$ drop of $\geq 1$ $\mathrm{mmol} / \mathrm{L}$, had a post-biopsy haemoglobin level of $5.6 \mathrm{mmol} / \mathrm{L}$ or greater and needed no interventions and were managed by observation only.

Table 4.2 Bleeding endpoint characteristics

\begin{tabular}{cc}
\hline Variable - no. (\%) & Biopsies with endpoint \\
\hline Composite clinically relevant bleeding complication & $17(11.0)^{\mathrm{a}}$ \\
Stadium I-II CKD & $1(7.7)^{\mathrm{b}}$ \\
Stadium III CKD & $8(13.1)^{\mathrm{b}}$ \\
Stadium IV CKD & $4(12.5)^{\mathrm{b}}$ \\
Stadium V CKD & $4(8.7)^{\mathrm{b}}$ \\
Major complication & $1(0.6)^{\mathrm{a}}$ \\
Intervention needed & $1(100)^{\mathrm{c}}$ \\
Hb drop $\geq 1$ mmol/L & $1(100)^{\mathrm{c}}$ \\
Ultrasound finding & $1(100)^{\mathrm{c}}$ \\
New haematuria & $0(0)^{\mathrm{c}}$ \\
Minor complication & $16(10.4)^{\mathrm{a}}$ \\
Intervention neeeded & $0(0)^{\mathrm{c}}$ \\
Hb drop $\geq 1$ mmol/L & $13(81.3)^{\mathrm{c}}$ \\
Ultrasound finding & $2(12.5)^{\mathrm{c}}$ \\
New haematuria & $4(25)^{\mathrm{c}}$ \\
\hline
\end{tabular}

*Proportions are not significantly different among the CKD stadia $(p>0.05)$

a $\%$ of all biopsies, ${ }^{b} \%$ of subjects within CKD stadium, ${ }^{c} \%$ of complication group

Table 4.2 gives an overview of bleeding endpoint characteristics. Composite endpoint of clinical relevant bleeding complication was defined as haemoglobin drop $\geq 1 \mathrm{mmol} / \mathrm{L}$, need for transfusion or intervention, positive ultrasound post-biopsy, or post-biopsy haematuria (>200RBC/HPF on microscopy) without pre-biopsy haematuria on microscopy. A major complication was defined as subjects needing a intervention (e.g. embolization, coiling, explantation, transfusion, bladder catherization). A minor complication was defined as all clinical relevant non-major bleeding complications.

Abbreviations: $\mathrm{CKD}$, chronic kidney disease; $\mathrm{Hb}$, hemoglobin; RBC/HPF, red blood cells per high power field 
Of all subjects, $35.1 \%$ had haematuria pre-biopsy. The majority of those $(87.0 \%)$ were scheduled for a non-surveillance biopsy. Four subjects had newly developed haematuria of $>200 \mathrm{RBC} / \mathrm{HPF}$ on urine analysis, but needed no bladder catheterization.

One of all subjects had a positive bleeding endpoint (haemoglobin drop of 1.0 $\mathrm{mmol} / \mathrm{L}$ ) after a previously uneventful biopsy in this study.

Besides the one transfusion, none of the subjects needed an intervention (e.g. embolization, coiling, or explantation of the kidney), had graft loss, or died within 30 days.

Table 4.3 Study parameters classified according to CKD stadium

\begin{tabular}{|c|c|c|c|c|c|}
\hline Variable - no. (\% of stadium) & Overall & $\begin{array}{l}\text { Stadium } \\
\text { I-II CKD }\end{array}$ & $\begin{array}{l}\text { Stadium } \\
\text { III CKD }\end{array}$ & $\begin{array}{l}\text { Stadium } \\
\text { IV CKD }\end{array}$ & $\begin{array}{l}\text { Stadium } \\
\text { V CKD }\end{array}$ \\
\hline Low MPV $(<9.2 \mathrm{fL})^{\mathrm{b}}$ & $105(78.9)$ & $9(90.0)$ & $50(89.3)^{\dagger}$ & $23(85.2)$ & $23(57.5)^{\dagger}$ \\
\hline Anaemia $\left(o^{-}<8.2 ; \circ<7.3 \mathrm{mmol} / \mathrm{L}\right)^{\mathrm{C}}$ & $96(63.2)$ & $2(15.4)^{*}$ & $23(37.7)^{*}$ & $25(78.1)^{\#}$ & $46(100)^{\#}$ \\
\hline Thrombocytopenia $\left(<130 \times 10^{9} / \mathrm{L}\right)^{a}$ & $8(5.3)$ & $0(0)$ & $2(3.3)$ & $1(3.1)$ & $5(10.9)$ \\
\hline Elevated urea $(>8 \text { to } 16 \mathrm{mmol} / \mathrm{L})^{b}$ & $67(44.4)$ & $1(7.7)^{\dagger, \pm}$ & $37(60.7)^{\dagger, \#}$ & $14(45.2)^{\ddagger}$ & $15(32.6)^{\#}$ \\
\hline High urea $(>16 \mathrm{mmol} / \mathrm{L})^{\mathrm{c}}$ & $43(28.5)$ & $0(0)^{*}$ & $0(0)^{*}$ & $14(45.2)^{* *}$ & $29(63.0)^{x+m}$ \\
\hline d Ivy BT (>4 min) $)^{a}$ & $8(5.4)$ & $0(0)$ & $2(3.3)$ & $1(3.2)$ & $5(11.4)$ \\
\hline d PFA-100 (at least one & $10(6$ & $0(0)$ & $4(6.9)$ & $0(0)$ & $6(13.6)$ \\
\hline Prolonged COL/ADP ${ }^{\mathrm{a}}$ & $6(4.0)$ & $0(0)$ & $1(1.7)$ & $0(0)$ & $5(10.9)$ \\
\hline גוסזו & 48 & $0(0)$ & $3(5.1)$ & $0(0)$ & $1(2.4)$ \\
\hline Reduced MEA (at least one) ${ }^{a}$ & $72(49.3)$ & $6(50.0)$ & $29(48.3)$ & $13(41.9)$ & $24(55.8)$ \\
\hline $\mathrm{ADP}^{\mathrm{a}}$ & $34(23$. & $4(33.3)$ & $9(15.0)$ & $7(22.6)$ & $14(32.6)$ \\
\hline Reduced ASPI ${ }^{\mathrm{a}}$ & $44(30.1)$ & $4(33.3)$ & $16(26.7)$ & $8(25.8)$ & $16(37.2)$ \\
\hline $\mathrm{COL}^{\mathrm{a}}$ & $44(30.1)$ & $4(33.3)$ & $21(35.0)$ & $8(25.8)$ & $11(25.6)$ \\
\hline Reduced TRAP ${ }^{a}$ & $20(13.7)$ & $2(16.7)$ & $5(8.3)$ & $7(22.6)$ & $6(14.0)$ \\
\hline
\end{tabular}

${ }^{a}$ Proportions are not significantly different among groups $(p>0.05)$

${ }^{\mathrm{b}}$ Significance levels for coupled proportions between CKD stadia: ${ }^{\dagger} p<0.01,{ }^{\ddagger}$ or ${ }^{\#} p<0.05$

${ }^{\mathrm{c}}$ All significantly different at $p<0.01$ (except coupled proportions ${ }^{*} p<0.05$, ${ }^{*}$ or ${ }^{* *} p>0.05$ )

Table 4.3 shows the study parameters classified according to CKD stadium before multiple imputation for missing data. Prolonged PFA-100 and reduced MEA were classified according to Kuiper et al. $^{20}$

Abbreviations: ADP, adenosine diphosphate; ASPI, arachidonic acid; BT, bleeding time; COL, collagen; MEA, multiple electrode aggregometry; CKD, chronic kidney disease; COL/ADP, collagen/adenosine diphosphate cartridge; COL/EPI, collagen/epinephrine cartridge; CT, closure time; $\mathrm{HT}$, haematocrit; L, litre; MEA, multiple electrode aggregometry; MPV, mean platelet volume; PFA100, Platelet Function Analyser 100; TRAP, thrombin receptor activating peptide-6

\section{Medication effects}

ASA use was reported in six of the subjects with a bleeding endpoint. The patient not having stopped ASA did not have a bleeding endpoint. Subjects reporting the use of ASA had a higher risk of at least one bleeding endpoint compared to those not using ASA (OR, 3.19; 95\% confidence interval (95\% Cl), 1.06 to 9.61). 

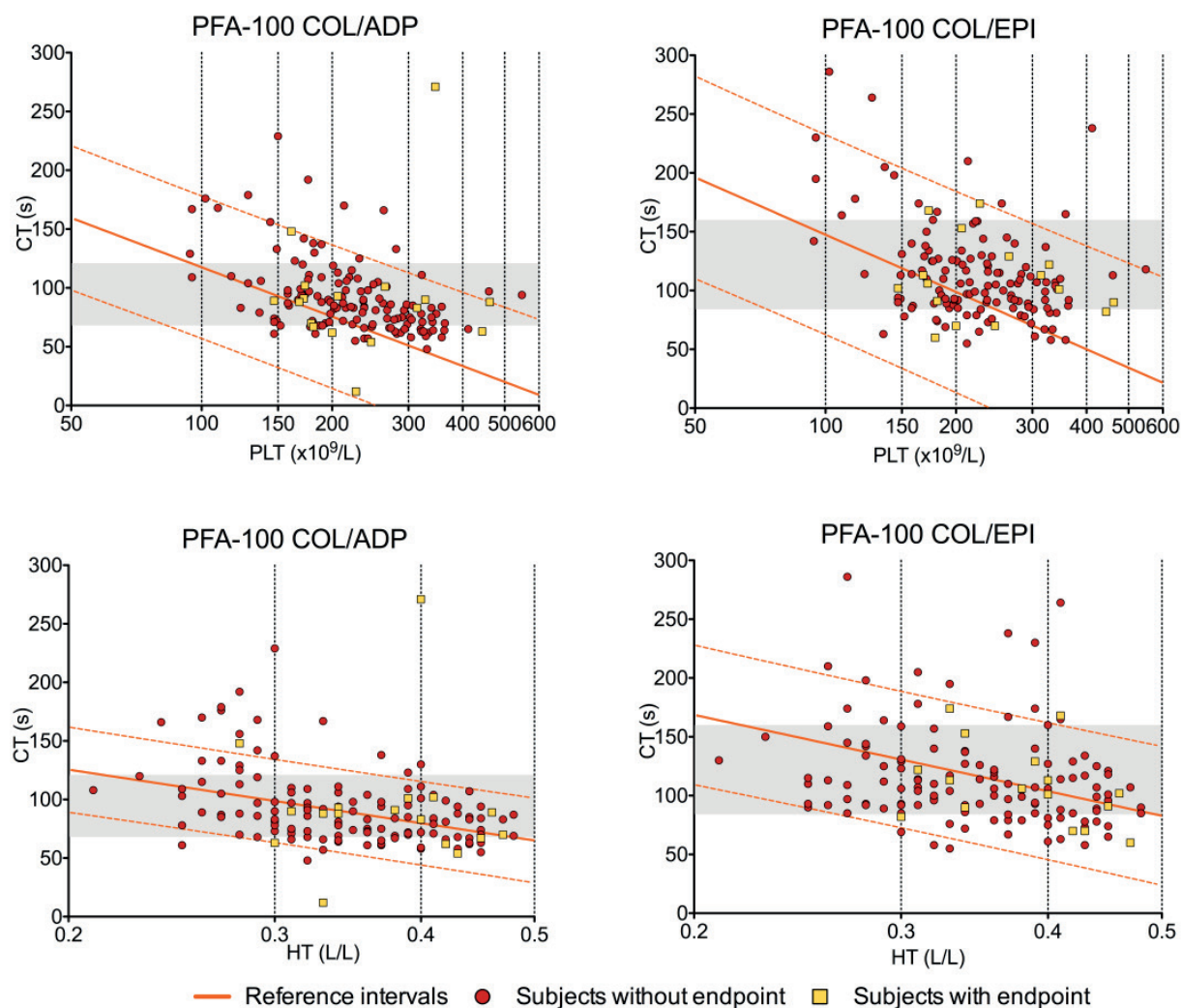

Figure 4.1 Pre-biopsy PFA-100 data corrected for thrombocyte count and haematocrit levels The grey areas are the originally defined reference intervals as advised by the manufacturer of PFA-100. The orange dotted lines are the newly defined reference intervals by Kuiper et al. ${ }^{20}$ Red dots depict results of the subjects without a bleeding endpoint. Yellow squares represent subjects with a bleeding endpoint.

Abbreviations: COL/ADP, collagen adenosine diphosphate cartridge; COL/EPI, collagen epinephrine cartridge; CT, closure time; HT, haematocrit; PFA-100, platelet function analyser 100; PLT, thrombocyte count

ASA use had an OR of $8.11(95 \% \mathrm{Cl}, 1.28$ to 51.4$)$ for prolonged PFA-100 COL/EPI using the newly defined reference intervals ${ }^{20}$ and an OR of 4.84 (95\% $\mathrm{Cl}, 1.60$ to 14.63$)$ using the original reference intervals.

ASA users did have a 2.41 OR for a reduced collagen activated on newly corrected $\mathrm{MEA}(95 \% \mathrm{Cl}, 1.00$ to 5.82$)$ and a $4.05 \mathrm{OR}$ for a reduced $\mathrm{ASPI}$ activated AUC on MEA when using the original reference intervals $(95 \% \mathrm{Cl}, 1.51$ to 10.83). 

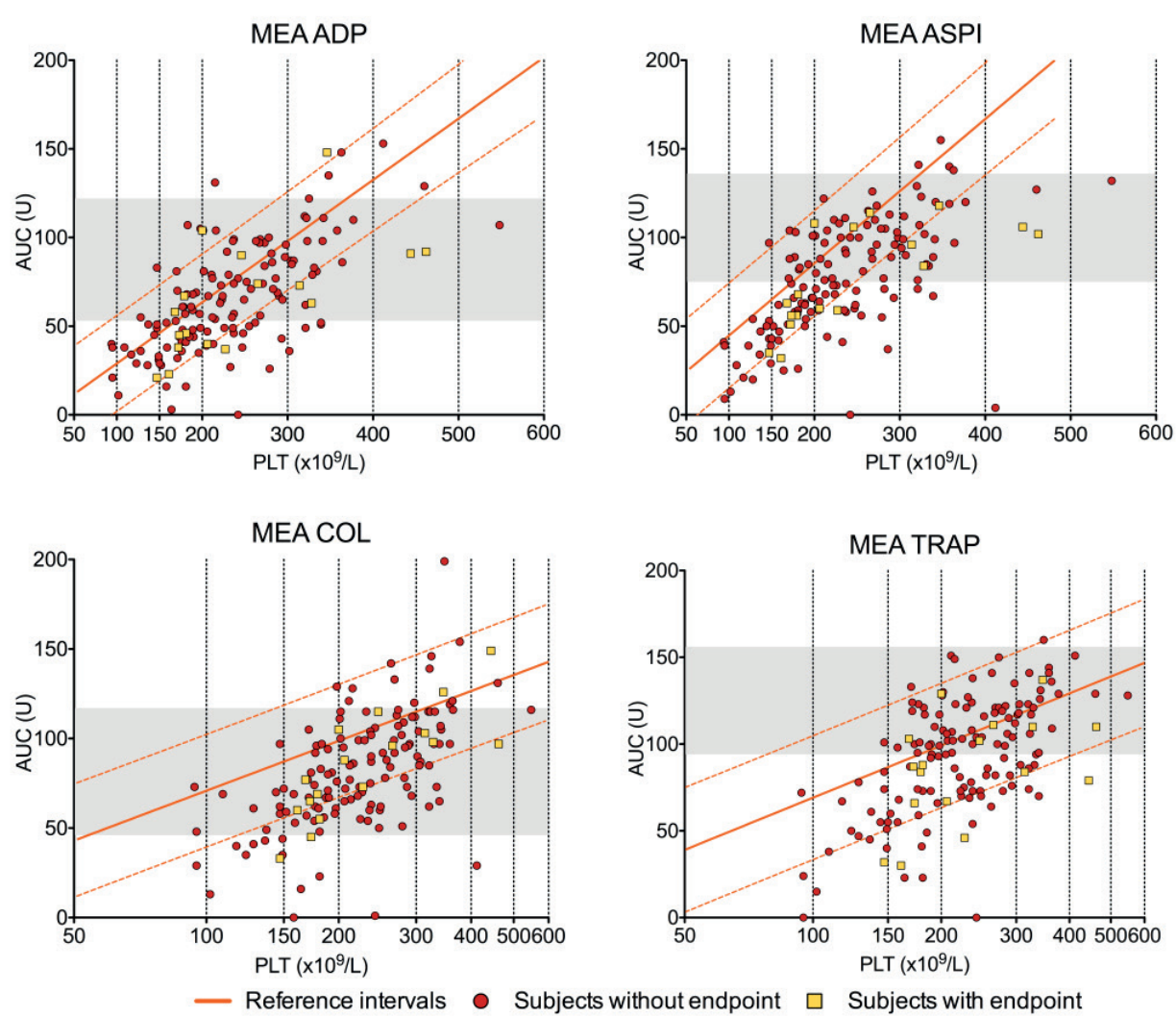

Figure 4.2 Pre-biopsy MEA data corrected for thrombocyte count

The grey areas are the originally defined reference intervals as advised by the manufacturer of Multiplate. The orange dotted lines are the newly defined reference intervals by Kuiper et al. ${ }^{20}$ Red dots depict results of subjects without a bleeding endpoint. Yellow squares represent subjects with a bleeding endpoint.

Abbreviations: ADP, adenosine diphosphate; ASPI, arachidonic acid; AUC, area under the curve; $\mathrm{COL}$, collagen; MEA, multiple electrode aggregometry; PLT, thrombocyte count; TRAP, thrombin receptor activating peptide-6

In five subjects PT-INR was elevated (>1.2). In these subjects median PT-INR was 1.4 (IQR 1.28-1.60). None of these subjects experienced a bleeding endpoint.

\section{Point-of-care testing of thrombocyte function and bleeding prediction}

The bleeding time was prolonged in eight subjects (bleeding time range: 243-

476 s), while in only one of these subjects a positive bleeding endpoint occurred. Bleeding time did not differ among different CKD stadia (table 4.3). 
For the PFA-100, six subjects had a prolonged COL/ADP test and four a prolonged COL/EPI test. Figure 4.1 has the PFA-100 results plotted for either correction of thrombocyte count or of haematocrit. One bleeding subject had a prolonged COL/ADP test, while none of the bleeding subjects had a prolonged COL/EPI test.

MEA was reduced in $23 \%$ of the subjects for ADP, $30 \%$ of the subjects for AS$\mathrm{PI}, 30 \%$ of the subjects for COL, and $14 \%$ of the subjects for TRAP (figure 4.2) for the newly defined reference intervals. ${ }^{20}$ The distribution among the CKD stadia did not differ for the number of subjects with reduced MEA results (table 4.3). Of all subjects almost half had a low AUC on at least one of the four MEA tests. Eight bleeding subjects had no test on MEA reduced, two had one test reduced, three had two tests reduced, four had three tests reduced, and none had all four tests reduced. This distribution was not significantly different among the five combinations possible. None of the four MEA tests had a significant OR for the prediction of a bleeding endpoint.

\section{Other predictors of bleeding complications}

Non-surveillance kidney biopsy had similar odds for a bleeding endpoint compared to surveillance biopsies (OR, 0.46; $95 \% \mathrm{Cl}, 0.15$ to 1.38).

Thrombocytopenia was evenly distributed among the CKD stadia (table 4.3). None of the bleeding subjects had thrombocytopenia.

A low MPV was seen less frequent in stadium V CKD in comparison to the other stadia (table 4.3). Reduced MPV did not give higher odds for a bleeding endpoint (OR, 1.82; 95\% Cl, 0.39 to 8.60).

Bleeding complications did not differ significantly among the different CKD stadia (table 4.3). As could be expected, urea levels were inversely related with eGFR (Pearson correlation coefficient, $r=-0.69 ; p<0.001$ ). Elevated (OR, $0.66 ; 95 \% \mathrm{Cl}, 0.23$ to 1.89 ) or high urea (OR, $0.93 ; 95 \% \mathrm{Cl}, 0.28$ to 3.10 ) did not prove to give higher odds ratio for a positive bleeding endpoint (OR, 0.87; $95 \% \mathrm{Cl}, 0.29$ to 2.65 when elevated and high urea were combined in comparison to normal urea level). 


\section{Chapter 4}

\section{Diagnostic performance of endpoint prediction}

Table 4.4 shows the data after multiple imputation on diagnostic performance of bleeding endpoint prediction when using bleeding time, the two PFA-100 cartridges (comparing the standard and newly defined reference intervals), all four MEA agonists (comparing the standard and newly defined reference intervals), and the MPV. PPV is low for all tests in our population, ranging from 0 to $17 \%$. NPV is between 88 and $93 \%$ for all tests.

Table 4.4 Diagnostic performance of all tests in predicting a bleeding endpoint

\begin{tabular}{|c|c|c|c|c|c|c|c|c|}
\hline Investigated test & Sensitivity & Specificity & AUC & PPV & NPV & PLHR & NLHR & $\mathrm{K}^{*}$ \\
\hline $\begin{array}{l}\text { BT } \\
\text { PFA-100 standard RI }\end{array}$ & 0.07 & 0.95 & 0.496 & 0.14 & 0.89 & 1.35 & 0.98 & NA \\
\hline COL/ADP & 0.12 & 0.87 & 0.490 & 0.10 & 0.89 & 0.89 & 1.02 & 0.04 \\
\hline COL/EPI & 0.15 & 0.88 & 0.474 & 0.13 & 0.89 & 1.25 & 0.97 & 0.48 \\
\hline \multicolumn{9}{|l|}{ PFA-100 new RI } \\
\hline COL/ADP & 0.06 & 0.96 & 0.494 & 0.16 & 0.89 & 1.55 & 0.98 & -0.07 \\
\hline COL/EPI & 0.00 & 0.95 & 0.529 & 0.00 & 0.88 & 0.00 & 1.05 & 0.00 \\
\hline \multicolumn{9}{|l|}{ MEA standard RI } \\
\hline ADP & 0.41 & 0.61 & 0.507 & 0.11 & 0.89 & 1.04 & 0.97 & 0.20 \\
\hline ASPI & 0.53 & 0.51 & 0.504 & 0.12 & 0.90 & 1.07 & 0.93 & 0.13 \\
\hline $\mathrm{COL}$ & 0.12 & 0.90 & 0.501 & 0.13 & 0.89 & 1.17 & 0.98 & -0.10 \\
\hline TRAP & 0.59 & 0.56 & 0.552 & 0.14 & 0.92 & 1.33 & 0.74 & 0.10 \\
\hline \multicolumn{9}{|l|}{ MEA new RI } \\
\hline ADP & 0.35 & 0.78 & 0.436 & 0.17 & 0.91 & 1.60 & 0.83 & 0.24 \\
\hline ASPI & 0.35 & 0.70 & 0.480 & 0.13 & 0.90 & 1.17 & 0.93 & 0.24 \\
\hline $\mathrm{COL}$ & 0.24 & 0.68 & 0.531 & 0.08 & 0.88 & 0.74 & 1.12 & -0.12 \\
\hline TRAP & 0.24 & 0.86 & 0.440 & 0.17 & 0.90 & 1.68 & 0.89 & 0.40 \\
\hline MPV & 0.87 & 0.22 & 0.532 & 0.12 & 0.93 & 1.12 & 0.59 & 0.02 \\
\hline
\end{tabular}

*Between BT and study tests in correctly predicting a positive endpoint

Table 4.4 portraits the diagnostic performance of all tests in predicting a bleeding endpoint after multiple imputation of missing data. New reference intervals were used from Kuiper et al. ${ }^{20}$

Abbreviations: ADP, adenosine diphosphate; ASPI, arachidonic acid; AUC, area under the receiver operating characteristic curve; BT, bleeding time; COL, collagen; COL/ADP, collagen adenosine diphosphate cartridge; COL/EPI, collagen epinephrine cartridge; $\mathrm{\kappa}$, kappa; MEA, multiple electrode aggregometry; MPV, mean platelet volume; NLHR, negative likelihood ratio; NPV, negative predictive value; PFA-100, platelet function analyser 100; PLHR, positive likelihood ratio; PPV, positive predictive value; RI, reference intervals; TRAP, thrombin receptor activating peptide-6

\section{Substitution of bleeding time by PFA-100}

Bland-Altman analysis between bleeding time and PFA-100 COL/ADP revealed a mean bias of 35.8 seconds (95\% limits of agreement, -95.7 to 167.3 seconds) with more dispersion at higher means. The same was apparent for the bleeding time and the PFA-100 COL/EPI cartridge (bias, 11.3 seconds; 95\% limits of agreement, -122.7 to 145.3 seconds). 
Because of higher dispersion at larger means, bias and 95\% limits of agreement were corrected for the mean. This gave for ADP/COL a bias of $27 \%(95 \%$ limits of agreement, $-71 \%$ to $125 \%$ ) and for ADP/EPI a bias of 5\% (95\% limits of agreement, $-92 \%$ to $102 \%$ ). Kappa values between bleeding time and both PFA-100 tests showed no agreement in correctly predicting a combined endpoint, except for the COL/EPI cartridge with the original reference intervals, which has moderate agreement (table 4.4).

\section{Discussion}

In this study we have investigated if bleeding complications after transplanted kidney biopsy could be predicted using Ivy bleeding time, MEA, PFA-100, and/or MPV. Furthermore we assumed that the PFA-100 would be superior than the bleeding time in predicting bleeding. None of our assumptions were true. We could not reliably predict bleeding complications after transplanted kidney biopsies by any of the tests. Prior ASA use, even after having stopped it for five days, was the only determinant of a bleeding complication.

Post-biopsy major bleeding complications with severe consequences are infrequent in patients after kidney transplantation. ${ }^{1-3}$ In our study 17 out of 154 subjects $(11.0 \%)$ had a clinically relevant bleeding event of which only one subject $(0.65 \%)$ had a severe haemoglobin drop and needed a transfusion. Most of the subjects $(9.1 \%)$ had a haemoglobin drop of more than $1 \mathrm{mmol} / \mathrm{L}$ without clinical consequences. Previously, 'macrohaematuria' was defined as more than 50 $\mathrm{RBC} / \mathrm{HPF},{ }^{28,29}$ but in our laboratory this cut off value is not reported and more than 200 RBC/HPF was used instead. New haematuria was seen in $2.6 \%$ of our subjects, which is slightly higher in comparison to other studies. ${ }^{1,3}$ Our conclusion is, that these bleeding endpoint data are coherent with previous studies. ${ }^{1-}$ $3,7,9,30$

Previous research has shown that antiplatelet and/or anticoagulant drugs should be stopped pre-biopsy, ${ }^{31}$ and that a thinner needle (i.e. a larger gauge) is important in reduction of complications. ${ }^{7}$ This and more research has led to evidence based recommendations for kidney biopsies in order to minimize bleeding complications. ${ }^{1}$ In our study, previously reported use of ASA was a risk 


\section{Chapter 4}

factor (three times higher odds) for having a bleeding endpoint. An ASA effect could still be identified using MEA (reduced AUC for the COL reagent) and PFA-100 (prolonged CT in the COL/EPI cartridge); stopping ASA five days before biopsy might be too short, however the one patient not having stopped its use did not have a bleeding event. Similarly, Mackinnon et al found that, if antiplatelet medication was continued, patients have a bigger drop in haemoglobin than when it was stopped five days before biopsy. ${ }^{31}$ Rates of major complications were not statistically different in their study. Interestingly, in the systematic review and meta-analysis of Corapi et al, the withdrawal of antiplatelet medication shorter than seven days did not result in more erythrocyte transfusion or macroscopic haematuria rates. ${ }^{7}$ As is discussed by Nayak-Rao the cessation of antiplatelet medication should be balanced between bleeding complications if continued and thrombotic complications when stopped. ${ }^{32}$ And it may be the case that the bleeding risk outweighs the thrombotic risk in our study.

When looking at PFA-100 results, only few subjects had a prolonged result when comparing to our newly defined reference intervals. This would indicate that hardly any of the subjects have a bleeding tendency, due to a platelet dysfunction. On the contrary, many subjects showed reduced MEA results (figure 4.2), which would indicate the presence of platelet dysfunction. Indeed, the stadium V CKD patients seem to be more prone to bleeding (table 4.3). They have significantly more anaemia due to renal dysfunction, higher grades of uraemia, and, although not significantly, more thrombocytopenia and more prolonged bleeding times. Renal capillary wall incompetence due to renal fibrosis is thought to be the main culprit causing haematuria in deteriorating CKD. ${ }^{16,33}$ Similarly is bleeding caused by anaemia, uremic toxins, or higher fibrinolytic activity in the urinary tract due to urokinase. ${ }^{16,33}$ However, in our study stadium V CKD did not result in higher rates of bleeding complications. Importantly, this is the first large study to date to report platelet dysfunction in CKD patients using a whole blood impedance aggregometer like MEA. Other researchers have drawn similar conclusions using a far less number of subjects and different measuring devices. ${ }^{34}$ 
No test in our study arsenal could adequately predict post-biopsy bleeding. The low PPV of all tests we found reflect that no test seems suitable for screening purposes. PFA-100 has properties similar to the bleeding time by incorporating flow into the analysis. However, Bland-Altman analysis showed that PFA-100 had bias in comparison to the bleeding time and could not give a meaningful verdict of the bleeding time because of large $95 \%$ limits of agreement in our population. Valeri and Ragno reported a similar finding, calculating correlation coefficients between the two tests. ${ }^{35}$ Prediction of bleeding complications seems difficult for kidney biopsies. Bleeding time (having a high specificity) could help in excluding bleeding complications. The PFA-100 has a similar high specificity of $96 \%$ and $95 \%$ for the COL/ADP and COL/EPI cartridge, respectively. The kappa value of 0.48 showed moderate agreement between the two tests in correctly predicting a bleeding outcome (COL/EPI original reference intervals versus the bleeding time). However, Islam and colleagues stated based on their results that PFA-100 is unlikely to be of benefit to patient care in routine kidney biopsies. ${ }^{36}$ Although similarities can be drawn between the bleeding time and the PFA-100, they are essentially different. ${ }^{37}$ The PFA-100 cannot be entitled as an in vitro bleeding time.

As a final conclusion we like to state that severe bleeding complications after kidney biopsy on transplanted kidneys are low; that laboratory tests (especially MEA) show that CKD patients have an in vitro platelet dysfunction on top of ASA use; that this acquired in vitro platelet dysfunction does not result in an increased bleeding risk after kidney biopsy, except possibly in ASA use; that the bleeding time could not be substituted by any other test we used; and that no test seems to able to adequately predict bleeding after kidney biopsy.

We recommend that routine haemostasis assessment before performing a transplanted kidney biopsy be abandoned after exclusion of patients with contra-indications. 


\section{Chapter 4}

\section{References}

1. Brachemi S, Bollée G. Renal biopsy practice: What is the gold standard? World J Nephrol. 2014 Nov 6;3(4):287-94.

2. Laute M, Vanholder R, Voet D, Peeters P, Cokelaere K, Deloose S, Terryn W. Safety and sample adequacy of renal transplant surveillance biopsies. Acta Clin Belg. 2013 May-Jun;68(3):1615.

3. Tøndel C, Vikse BE, Bostad L, Svarstad E. Safety and complications of percutaneous kidney biopsies in 715 children and 8573 adults in Norway 1988-2010. Clin J Am Soc Nephrol. 2012 Oct;7(10):1591-7.

4. Eiro M, Katoh T, Watanabe T. Risk factors for bleeding complications in percutaneous renal biopsy. Clin Exp Nephrol. 2005 Mar;9(1):40-5.

5. Shidham GB, Siddiqi N, Beres JA, Logan B, Nagaraja HN, Shidham SG, Piering WF. Clinical risk factors associated with bleeding after native kidney biopsy. Nephrology. 2005 Jun;10(3):305-10.

6. Ishikawa E, Nomura S, Hamaguchi T, Obe T, Inoue-Kiyohara M, Oosugi K, Katayama K, Ito M. Ultrasonography as a predictor of overt bleeding after renal biopsy. Clin Exp Nephrol. 2009 Aug;13(4):325-31.

7. Corapi KM, Chen JL, Balk EM, Gordon CE. Bleeding complications of native kidney biopsy: a systematic review and meta-analysis. Am J Kidney Dis. 2012 Jul;60(1):62-73.

8. Torres Muñoz A, Valdez-Ortiz R, González-Parra C, Espinoza-Dávila E, Morales-Buenrostro LE, Correa-Rotter R. Percutaneous renal biopsy of native kidneys: efficiency, safety and risk factors associated with major complications. Arch Med Sci. 2011 Oct;7(5):823-31.

9. Whittier WL, Korbet SM. Timing of complications in percutaneous renal biopsy. J Am Soc Nephrol. 2004 Jan;15(1):142-7.

10. Lehman CM, Blaylock RC, Alexander DP, Rodgers GM. Discontinuation of the bleeding time test without detectable adverse clinical impact. Clin Chem. 2001;47(7):1204-11.

11. Soyoral YU, Demir C, Begenik H, Esen R, Kucukoglu ME, Aldemir MN, Demirkiran D, Erkoc R. Skin bleeding time for the evaluation of uremic platelet dysfunction and effect of dialysis. Clin Appl Thromb Hemost. 2012 Mar-Apr;18(2):185-8.

12. Lutz J, Menke J, Sollinger D, Schinzel H, Thürmel K. Haemostasis in chronic kidney disease. Nephrol Dial Transplant. 2014 Jan;29(1):29-40.

13. Korbet SM, Volpini KC, Whittier WL. Percutaneous renal biopsy of native kidneys: a singlecenter experience of 1,055 biopsies. Am J Nephrol. 2014;39(2):153-62.

14. Peterson P, Hayes TE, Arkin CF, Bovill EG, Fairweather RB, Rock WA Jr, Triplett DA, Brandt JT. The preoperative bleeding time test lacks clinical benefit: College of American Pathologists' and American Society of Clinical Pathologists' position article. Arch Surg. 1998 Feb;133(2):1349.

15. Steiner RW, Coggins C, Carvalho AC. Bleeding time in uremia: a useful test to assess clinical bleeding. Am J Hematol. 1979;7(2):107-17. 
16. Mannucci PM, Tripodi A. Hemostatic defects in liver and renal dysfunction. Hematology Am Soc Hematol Educ Program. 2012;2012:168-73.

17. Gangji AS, Sohal AS, Treleaven D, Crowther MA. Bleeding in patients with renal insufficiency: a practical guide to clinical management. Thromb Res. 2006;118(3):423-8.

18. Manno C, Bonifati C, Torres DD, Campobasso N, Schena FP. Desmopressin acetate in percutaneous ultrasound-guided kidney biopsy: a randomized controlled trial. Am J Kidney Dis. 2011 Jun;57(6):850-5.

19. Van den Hoogen MW, Verbruggen BW, Polenewen R, Hilbrands LB, Nováková IR. Use of the platelet function analyzer to minimize bleeding complications after renal biopsy. Thromb Res. 2009;123(3):515-22.

20. Kuiper GJ, Houben R, Wetzels RJ, Verhezen PW, van Oerle R, ten Cate H, Henskens YM, Lancé MD. The use of regression analysis in determining reference intervals for low hematocrit and thrombocyte count in multiple electrode aggregometry and platelet function analyzer 100 testing of platelet function. Platelets. 2017 Jan 9:1-8.

21. Quiroga T, Mezzano D. Is my patient a bleeder? A diagnostic framework for mild bleeding disorders. Hematology Am Soc Hematol Educ Program. 2012;2012:466-74.

22. Harrison P. The role of PFA-100 testing in the investigation and management of haemostatic defects in children and adults. Br J Haematol. 2005 Jul;130(1):3-10.

23. Lancé MD, Sloep M, Henskens YM, Marcus MA. Mean platelet volume as a diagnostic marker for cardiovascular disease: drawbacks of preanalytical conditions and measuring techniques. Clin Appl Thromb Hemost. 2012 Nov; 18(6):561-8.

24. Bessman JD, Williams LJ, Gilmer PR Jr. Mean platelet volume. The inverse relation of platelet size and count in normal subjects, and an artifact of other particles. Am J Clin Pathol. 1981 Sep;76(3):289-93.

25. Levey AS, Bosch JP, Lewis JB, Greene T, Rogers N, Roth D. A more accurate method to estimate glomerular filtration rate from serum creatinine: a new prediction equation. Modification of Diet in Renal Disease Study Group. Ann Intern Med. 1999 Mar 16;130(6):461-70.

26. National Kidney Foundation. K/DOQI clinical practice guidelines for chronic kidney disease: evaluation, classification, and stratification. Am J Kidney Dis. 2002 Feb;39(2 Suppl 1):S1-266.

27. Mielke CH Jr, Kaneshiro MM, Maher IA, Weiner JM, Rapaport SI. The standardized normal Ivy bleeding time and its prolongation by aspirin. Blood. 1969 Aug;34(2):204-15.

28. Loo RK, Lieberman SF, Slezak JM, Landa HM, Mariani AJ, Nicolaisen G, Aspera AM, Jacobsen SJ. Stratifying risk of urinary tract malignant tumors in patients with asymptomatic microscopic hematuria. Mayo Clin Proc. 2013 Feb;88(2):129-38.

29. Hageman N, Aronsen T, Tiselius HG. A simple device (Hemostick) for the standardized description of macroscopic haematuria: our initial experience. Scand J Urol Nephrol. 2006;40(2):14954.

30. Schwarz A, Gwinner W, Hiss M, Radermacher J, Mengel M, Haller H. Safety and adequacy of renal transplant protocol biopsies. Am J Transplant. 2005 Aug;5(8):1992-6. 


\section{Chapter 4}

31. Mackinnon B, Fraser E, Simpson K, Fox JG, Geddes C. Is it necessary to stop antiplatelet agents before a native renal biopsy? Nephrol Dial Transplant. 2008 Nov;23(11):3566-70.

32. Nayak-Rao S. Percutaneous native kidney biopsy in patients receiving antiplatelet agents- is it necessary to stop them routinely? Indian J Nephrol. 2015 May-Jun;25(3):129-32.

33. Suárez-Álvarez B, Liapis $\mathrm{H}$, Anders HJ. Links between coagulation, inflammation, regeneration, and fibrosis in kidney pathology. Lab Invest. 2016 Apr;96(4):378-90.

34. Zeck J, Schallheim J, Lew SQ, DePalma L. Whole blood platelet aggregation and release reaction testing in uremic patients. Biomed Res Int. 2013;2013:486290.

35. Valeri CR, Ragno G. In vitro testing of platelets using the thromboelastogram, platelet function analyzer, and the clot signature analyzer to predict the bleeding time. Transfus Apher Sci. 2006 Aug;35(1):33-41.

36. Islam N, Fulop T, Zsom L, Miller E, Mire CD, Lebrun CJ, Schmidt DW. Do platelet function analyzer-100 testing results correlate with bleeding events after percutaneous renal biopsy? Clin Nephrol. 2010 Mar;73(3):229-37.

37. Ho SJ, Gemmell R, Brighton TA. Platelet function testing in uraemic patients. Hematology. 2008 Feb;13(1):49-58. 


\section{Chapter 5}

\section{Validation of a modified}

thromboelastometry approach

to detect changes in

fibrinolytic activity

Kuiper GJ, Kleinegris MC, van Oerle R, Spronk HM, Lancé MD, ten Cate $\mathrm{H}$, Henskens YM

Thromb J. 2016 Jan 14;14:1. 


\section{Abstract}

\section{Background}

Thus far, validated whole blood assays used in in vitro fibrinolysis experiments using thromboelastometry (ROTEM) are lacking or have yet to be tested in humans. The objective was first, to establish a standardized modified ROTEM approach to detect both hypo- and hyperfibrinolysis. And second, to perform a technical and clinical validation of the assay.

\section{Methods}

Blood was used of healthy volunteers, patients with sepsis, patients after cardiothoracic surgery, pregnant women, and cirrhotic liver disease patients. A whole blood tissue factor (TF) activated ROTEM assay with and without the addition of recombinant tissue plasminogen activator (rTPA) was developed. Plasma fibrinolysis determinants were measured in all volunteers and patients.

\section{Results}

Thirty five pM TF and additions of 125 and $175 \mathrm{ng} / \mathrm{ml} \mathrm{rTPA}$ resulted in full lysis within 60 min in healthy volunteers. Coefficients of variation were below $10 \%$ without and below $20 \%$ with rTPA addition. In sepsis the hypofibrinolytic ROTEM profiles with $175 \mathrm{ng} / \mathrm{ml}$ rTPA were in line with the plasma determinants (high PAI-1, high fibrinogen, low tPA activity, and high D-dimers). After cardiothoracic surgery, reduced fibrinogen and platelet levels accounted for the reduced maximum clot firmness. The hypofibrinolytic profile is attributed to tranexamic acid use and elevated PAI-1 levels. The lowest rTPA concentration in cirrhosis resulted in hyperfibrinolysis in only few of the patients. In pregnancy normal profiles were found.

\section{Discussion}

Our high rTPA concentration demonstrates hypofibrinolytic profiles adequately in sepsis and after cardiothoracic surgery. Our low rTPA concentration of $125 \mathrm{ng} / \mathrm{ml}$ seems too high for demonstrating hyperfibrinolysis in cirrhotic liver disease.

\section{Conclusions}

We were able to present a validated whole blood ROTEM approach to fibrinolysis testing using added rTPA, which can be of added value next to classical plasma based fibrinolysis assays. 


\section{Chapter 5}

\section{Background}

Fibrinolysis is the process of degradation of a fibrin clot into so-called fibrin degradation products (FDPs) of which the D-dimers are best known. Following coagulation, tissue-type plasminogen activator (tPA) induces fibrinolysis by activation of plasminogen into plasmin which enzymatically degrades fibrin into FDPs. To avoid excess clot lysis activity, the fibrinolytic system is under tight control of alpha 2-antiplasmin, plasminogen activator inhibitor-1 (PAl-1), and thrombin activated fibrinolysis inhibitor (TAFI). However, a disbalance in pro- and antifibrinolytic factors can result in either hyper- or hypofibrinolysis, which may contribute to the risk for bleeding or thrombosis, respectively. In trauma and cirrhosis the increased bleeding tendency is attributed to a hyperfibrinolytic state in combination with a rebalanced haemostatic system. ${ }^{1,2}$ In pregnancy, a hypercoaguable state may occur in conjunction with reduced fibrinolysis. ${ }^{3}$ In sepsis, the initial procoagulant state is associated with attenuated fibrinolysis due to elevated PAl-1, which may advance towards an uncontrolled syndrome of disseminated intravascular coagulation (DIC), with a clinical bleeding tendency. ${ }^{4,5}$ Factor XIII (FXIII) activated by thrombin crosslinks fibrin strands in order to strengthen the fibrin clot. Low FXIII activity levels are seen in sepsis and could contribute to an enhanced bleeding diathesis in severe septic shock. ${ }^{6}$

One of the therapeutic approaches to diminish overt fibrinolysis is treatment with tranexamic acid, which directly inhibits the action of plasmin. For instance, in cardiothoracic surgery this antifibrinolytic drug is being used to prevent and counteract extracorporeal circulation (ECC) induced hyperfibrinolysis, ${ }^{7}$ whereas in trauma the drug is used to diminish the bleeding tendency. ${ }^{8}$

Both the diagnosis of the fibrinolytic state and the management of therapeutic interventions aimed at the fibrinolytic system could benefit from a sensitive but also robust laboratory assay. Although most proteins and enzymes involved in fibrinolysis can be quantified, these do not properly reflect the dynamic status of the fibrinolytic system. Assays developed to detect hypo- or hyperfibrinolysis 


\section{Chapter 5}

are not commonly used in daily practice because they are cumbersome, plasma-based and/or not suitable for bedside assessment. ${ }^{9}$ Several groups have modified overall haemostatic assays such that they would become more sensitive to changes in the fibrinolytic system. This was achieved by modifying whole blood thrombelastography $(T E G)^{10}$ and rotational thromboelastometry (ROTEM) ${ }^{11}$ by addition of recombinant TPA (rTPA) to induce fibrinolysis ex vivo. More research has been done using other methods or in specific patient groups. ${ }^{12-21}$ Overall, however, these studies lacked clinical validation in healthy volunteers as well as defined patient subsets with known abnormalities in coagulation and/or fibrinolysis.

The goal for our modified ROTEM test was to use whole blood, be tissue factor activated, have one application with and one without the addition of rTPA, and would have similar reproducibility in comparison to the commercial non-modified ROTEM tissue factor activated test (EXTEM). Ideally, it would have short(er) runtimes in comparison to plasma based fibrinolysis assays or markers, have good distinction between healthy volunteers and patient groups, be easy to interpret, and have results in line with plasma based markers for fibrinolysis. In this report we describe the development, the technical, and the clinical validation of a novel whole blood ROTEM-based assay application including rTPA to induce fibrinolysis.

\section{Methods}

\section{Group selection}

The institutional Ethics Committee of the Maastricht UMC+ approved this study and of all subjects informed consent was obtained. No compensation was given for participation in this study. Blood of healthy volunteers was used to validate the assay and to define reference intervals, while the blood of 4 patient groups (sepsis, cardiothoracic surgery (CTS), $3^{\text {rd }}$ trimester pregnancy, and cirrhotic liver disease Child-Pugh score B or $\mathrm{C}$ ) was used for clinical validation. Any notion of anticoagulation use in a subject was reason to exclude this subject from the study. Septic patients were recruited within 1 hour after the onset of sepsis as judged by the intensive care doctor in charge according to current guidelines ${ }^{22}$ 
after which prompt blood withdrawal was executed. Only septic patients without signs of (non-)overt DIC were included. ${ }^{23}$ Cardiothoracic surgery patients were recruited before surgery. Directly after the surgery upon arrival at the ICU blood withdrawal was done promptly. Patients receiving heparin were antagonized with protamine in a 1:1 ratio before arrival on the ICU according to local protocol. Females in their $3^{\text {rd }}$ trimester of pregnancy were recruited at the policlinic during regular check-ups or at the ward before having elective caesarean section the next day. Child-Pugh score $\mathrm{B}$ and $\mathrm{C}$ cirrhosis patients were recruited via the policlinic or at the ward after which blood was withdrawn.

\section{Preparation}

Human recombinant tissue factor (Innovin, Dade Behring Marburg GmbH, Marburg, Germany) was reconstituted according to the manufacturer's instructions obtaining a $1 \mathrm{IU} / \mathrm{mL}$ concentration. This TF solution together with $\mathrm{CaCl}_{2}$ at $1 \mathrm{M}$ and HN-Buffer (20 mM HEPES, $150 \mathrm{mM} \mathrm{NaCl}, \mathrm{pH}$ 7.7) was prepared in advance and kept at $4-7^{\circ} \mathrm{C}$ till further use. Recombinant tPA (Actilyse, Boehringer Ingelheim, Alkmaar, the Netherlands) was reconstituted according to the manufacturer's instructions ( $1 \mathrm{mg} / \mathrm{mL}$ or $580,000 \mathrm{IU} / \mathrm{mL}$ alteplase), immediately aliquoted, frozen, and kept at $-80{ }^{\circ} \mathrm{C}$ till further use. The blood withdrawal was performed by venepuncture discarding the first $3 \mathrm{~mL}$ or, if a radial artery catheter was in situ, by flushing the first $5 \mathrm{~mL}$ before the blood was collected in vacuum tubes containing 3.2\% (w/v) citrate (Vacutainer, Becton Dickinson BV, Breda, the Netherlands), $11 \mathrm{mM}$ citrate with $50 \mu \mathrm{g} / \mathrm{mL}$ corn trypsin inhibitor (CTI, Haematologic Technologies Inc., Essex Junction, VT, USA) and 0.5 M citrate at pH 4.3 (TriniLIZE Stabilyte, Tcoag Ireland Limited, Bray, Ireland). Tubes were gently mixed directly after blood withdrawal.

\section{ROTEM analysis}

The ROTEM device and cups and pins were from TEM International GmbH (Munich, Germany). The device temperature was set to $37{ }^{\circ} \mathrm{C}$ and the maximum runtime to $120 \mathrm{~min}$. TF, $\mathrm{CaCl}_{2}$, and rTPA (if applicable) were added to 300 $\mu \mathrm{L}$ whole blood to obtain final concentrations of $35 \mathrm{pM} \mathrm{TF}, 10 \mathrm{mM} \mathrm{CaCl}_{2}$, and 0 , 


\section{Chapter 5}

125 , and $175 \mathrm{ng} / \mathrm{mL}$ rTPA (which equals $0,72.5$, and $101.5 \mathrm{IU} / \mathrm{mL}$ alteplase). Total volume of the reagents was maintained at $50 \mu \mathrm{L}$ per cup by applying adequate volumes of $\mathrm{HN}$-buffer. The following standard ROTEM parameters were analysed per channel: CT (clotting time in seconds), MCF (maximum clot firmness in millimetres), LOT (lysis onset time in minutes), and LT (lysis time in minutes). The following derived parameters were calculated using the ROTEM data: the FS (fibrinolysis speed, defined as the decline in $\% / m i n$ between the LOT and LT points; thus: $F S=75 /\left(\mathrm{LT}_{\min }-\mathrm{LOT}_{\min }\right)$ ) and the $\mathrm{FS}_{\mathrm{c}}$ (the corrected fibrinolysis speed in $\mathrm{mm} / \mathrm{min}$ between the LOT and LT points; thus: $\mathrm{FS}_{\mathrm{c}}=(\mathrm{am}-$ plitude $_{\mathrm{LOT}}$ - amplitude $\left.\left.\mathrm{LT}_{\mathrm{T}}\right) /\left(\mathrm{LT} \mathrm{T}_{\min }-\mathrm{LOT}_{\min }\right)\right)$.

\section{Euglobulin clot lysis time analysis (ECLT)}

The euglobulin clot lysis time was performed similar to Kowalski. ${ }^{24}$ Differently, the precipitate was dissolved in $1 \mathrm{ml}$ Veronal buffer and the reaction was started by adding $25 \mu \mathrm{l}$ of thrombin $100 \mathrm{U} / \mathrm{ml}$. Every $10 \mathrm{~min}$ resolution of the clot was assessed visually for the first two hours. Afterwards it was observed every 15 min up to two hours. If a clot was still present at two hours, a last observation was made at four hours.

\section{Complete blood count}

Haemoglobin, haematocrit, platelet, and white blood cell counts were performed on a Sysmex XE-5000 (Sysmex Nederland B.V., Etten-Leur, The Netherlands) using citrated whole blood. Final values were adjusted for the extra $1 / 9^{\text {th }}$ volume of citrate present during the measurements.

\section{Preparation of platelet poor plasma (PPP)}

Blood was spun twice to gather PPP: a first step at $2,500 \mathrm{~g}$ for $5 \mathrm{~min}$, followed by a second step at $10,000 \mathrm{~g}$ for $10 \mathrm{~min}$. The PPP was directly aliquot, frozen, and kept at $-80^{\circ} \mathrm{C}$ till further use.

PT (prothrombin time), aPTT (activated partial thromboplastin time), alpha 2antiplasmin activity, plasminogen activity, Factor XIII activity, D-dimer level, and 
fibrinogen level

A BCS XP System (Siemens Healthcare Diagnostic B.V., The Hague, The Netherlands) was used for determining PT (Dade Innovin), aPTT (Dade actin), alpha 2-antiplasmin activity (Berichrom $\alpha 2$-Antiplasmin), plasminogen activity (Berichrom Plasminogen), factor XIII activity (Berichrom Factor XIII), D-dimer levels (Innovance D-Dimer), and fibrinogen levels (Multifibrin $U$ ) in PPP according to the manufacturer's instructions in batches.

tPA activity, PAI-1 activity, and TAFI antigen

ELISA kits for measuring tPA activity (ZYMUPHEN tPA Activity), PAl-1 activity (ZYMUTEST PAI-1 Activity), and TAFI antigen (ZYMUTEST proTAFI Ag) were acquired from Hyphen Biomed (Neuville-Sur-Oise, France) and measured in PPP according to the accompanied instructions in batches.

\section{Statistical analysis}

Data were collected and analysed with IBM SPSS Statistics v19.0.0 (IBM Corporation, Armonk, NY, USA). Categorical data are presented as $\mathrm{n}(\%)$, continuous data as median and inter quartile range (IQR). For reproducibility, coefficients of variation (CV's) are expressed as the ratio of the standard deviation to the mean. Between run CV's were calculated using 16 repeated measurements with blood of one withdrawal of one healthy volunteer. For $0 \mathrm{ng} / \mathrm{mL} \mathrm{rTPA} C \mathrm{~T}$ and MCF values were analysed and for 125 and $175 \mathrm{ng} / \mathrm{mL}$ rTPA LOT and LT values besides the CT and MCF. Furthermore within run CV's were calculated from 11 different blood withdrawals in one person over a time span of six months. In this case CT, MCF, LOT, and LT were assessed at $175 \mathrm{ng} / \mathrm{ml} \mathrm{rTPA}$. Optimal time from blood withdrawal to measurement was assessed by using blood of three healthy volunteers and keeping it at $37^{\circ} \mathrm{C}$ till further use. CT, MCF, LOT, and LT were measured using the third condition (i.e. $175 \mathrm{ng} / \mathrm{mL}$ rTPA) at $30 \mathrm{~min}, 1 \mathrm{~h}, 2 \mathrm{~h}, 3 \mathrm{~h}$, and $4 \mathrm{~h}$ after blood withdrawal and assessed for stability. For each time point a new vial of $\mathrm{CaCl}_{2}$, TF, and rTPA was used. Ninety five per cent reference ranges were determined using MedCalc v12.0 (MedCalc Software, Mariakerke, Belgium) using data from healthy volunteers. 


\section{Chapter 5}

Skewed data were normalized first and afterwards transformed back when calculating the $95 \%$ reference ranges. Mann-Whitney-U was used for comparison testing. Determinants of the ROTEM parameters and their 95\% confidence interval $(95 \% \mathrm{Cl})$ were calculated by multiple linear regression analysis assuming $p<0.05$ as statistically significant. Figures were constructed using Graphpad Prism v5.0a (GraphPad Software, San Diego, CA, USA).

\section{Results}

\section{Technical validation}

Before establishing the optimal concentrations for the rTPA induced fibrinolysis ROTEM assay, various configurations of TF and ITPA concentrations were investigated. This revealed that TF concentrations below $35 \mathrm{pM}$ resulted in prolonged CT values, whereas the MCF was hardly influenced (data not shown). Therefore, the final TF concentration was set at $35 \mathrm{pM}$. In blood from healthy volunteers, full clot lysis was obtained at a final rTPA concentration of 125 $\mathrm{ng} / \mathrm{mL}$ around $60 \mathrm{~min}$, while at a final concentration of $175 \mathrm{ng} / \mathrm{mL} \mathrm{rTPA}$ full lysis was observed around $45 \mathrm{~min}$ (data not shown). These concentrations were appropriate to maintain acceptable short runtimes. At both 125 and $175 \mathrm{ng} / \mathrm{mL}$ ITPA, ROTEM was characterized by a lower MCF compared to the assay without tPA (125 ng/mL rTPA: 2 mm (a 3.6\% decrease) and $175 \mathrm{ng} / \mathrm{mL}$ rTPA: $5 \mathrm{~mm}$ (an $8.4 \%$ decrease)), while the CT prolonged by $5 \mathrm{~s}$ (an 11\% increase) and $7 \mathrm{~s}$ (a 17\% increase) respectively in comparison to the assay without rTPA.

Assessment of reproducibility for the different ROTEM parameters showed between run CV's of $9.5 \%$ and $1.8 \%$ for the CT and MCF at the $0 \mathrm{ng} / \mathrm{mL}$ rTPA concentration. Between run CV's were $8.7 \%, 4.1 \%, 12.1 \%$ and $18.8 \%$ for CT, MCF, LOT, and LT at the $125 \mathrm{ng} / \mathrm{mL}$ rTPA concentration and for $175 \mathrm{ng} / \mathrm{mL}$ rTPA CV's were $12.1 \%, 4.9 \%, 16.6 \%$ and $17.1 \%$ for each parameter, respectively. Within run CV's showed $25 \%$ for CT, $8.4 \%$ for MCF, $16.0 \%$ for LOT, and $27.2 \%$ for LT at $175 \mathrm{ng} / \mathrm{ml}$ rTPA. To determine whole blood stability in the assay, ROTEM analysis was started at 30, 60, 120, 180, and 240 min after blood drawing. At $175 \mathrm{ng} / \mathrm{mL}$ rTPA, all ROTEM derived parameters remained stable 
up to $4 \mathrm{~h}$ after sample collection (figure 5.1). Comparable results were obtained for the assay in the presence of $125 \mathrm{ng} / \mathrm{mL}$ rTPA (data not shown).

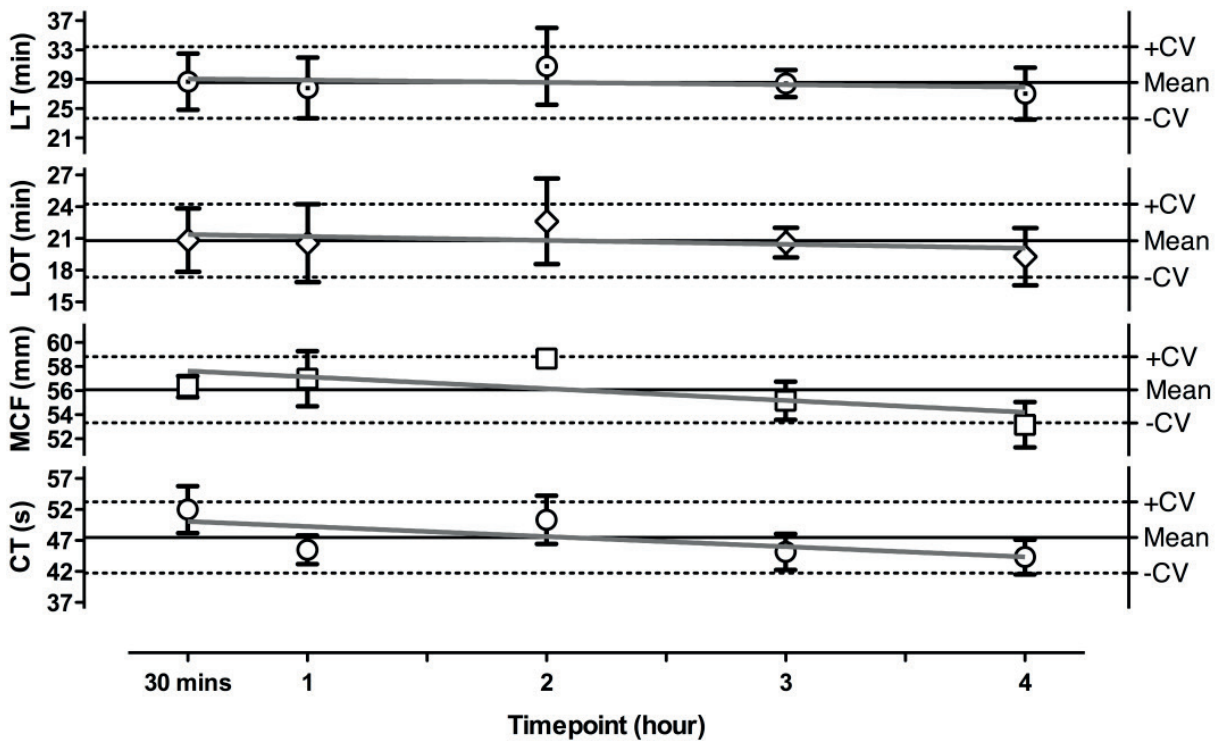

Figure 5.1 Whole blood stability when performing rTPA induced fibrinolysis on a ROTEM. Whole blood stability at room temperature (at 0.5 to $4 \mathrm{~h}$ after blood drawing) of 3 healthy volunteers for ROTEM parameters at $175 \mathrm{ng} / \mathrm{mL}$ rTPA; mean per time point ( \pm SEM); overall mean (solid horizontal line on $\mathrm{Y}$-axis) \pm error margin based on $\mathrm{CV}$ of test (dotted horizontal lines on $\mathrm{Y}$-axis); fitted line through all value points (grey declining line)

Abbreviations: CT, clotting time; CV, correlation of variation; MCF, maximum clot firmness; LOT, lysis onset time; LT, lysis time

\section{Reference intervals}

ROTEM analysis data from 40 healthy volunteers (male to female ratio of $1: 1$ ) was used to calculate the 95\% reference intervals (table 5.1). Age, height, body weight, haemoglobin, and haematocrit level were higher in males compared to females (mean difference: 25 years, $14 \mathrm{~cm}, 27.5 \mathrm{~kg}, 1.1 \mathrm{mmol} / \mathrm{L}$, and $0.04 \mathrm{~L} / \mathrm{L}$, respectively: all $p<0.05)$ while platelet count, white blood cell count, and fibrinogen levels were not different between the two sexes. Regarding the ROTEM parameters, CT values were significantly different between the two sexes with males having shorter CTs for all rTPA concentrations $(6.5,12.8$, and $14.5 \mathrm{~s}$ at 0 , 125, and $175 \mathrm{ng} / \mathrm{mL}$ rTPA respectively: all $p<0.05)$. Differences between males and females were not observed for the other ROTEM parameters: i.e. MCF, LOT, and LT for any of the three conditions. 
Table 5.1 95\% reference intervals for tissue factor stimulated ROTEM parameters in whole blood of 40 healthy volunteers using different concentrations of rTPA.

\begin{tabular}{lccc}
\hline & $0 \mathrm{ng} / \mathrm{mL} \mathrm{rTPA}$ & $125 \mathrm{ng} / \mathrm{mL} \mathrm{rTPA}$ & $175 \mathrm{ng} / \mathrm{mL} \mathrm{rTPA}$ \\
\hline CT $(\mathrm{s})$ & $36-72^{*}$ & $33-75^{*}$ & $29-73^{*}$ \\
MCF $(\mathrm{mm})$ & $52-70^{*}$ & $47-68^{*}$ & $39-66^{*}$ \\
LOT $(\mathrm{min})$ & $73-120$ & $26-49^{*}$ & $18-40^{*}$ \\
LT $(\mathrm{min})$ & $>120$ & $35-77^{*}$ & $25-57^{*}$ \\
FS $(\% / \mathrm{min})$ & NA & $2.5-9.8^{*}$ & $4.1-12.0$ \\
FS $_{\mathrm{c}}(\mathrm{mm} / \mathrm{min})$ & $\mathrm{NA}$ & $1.4-5.7^{*}$ & $2.4-6.6^{*}$ \\
\hline
\end{tabular}

*after normalization and transformation

Abbreviations: rTPA, recombinant tissue plasminogen activator; CT, clotting time; MCF, maximum clot firmness; LOT, lysis onset time; LT, lysis time; FS, fibrinolysis speed, defined as the decline in $\% /$ minute between the LOT and LT points thus: $F S=75 /\left(L T_{\min }-L_{\text {OOT }}\right)$; $F S_{c}$, corrected fibrinolysis speed in $\mathrm{mm} /$ minute between the LOT and LT points thus: $F S_{c}=\left(A_{L o T}-A_{L T}\right) /\left(L T_{\min }-L O T_{\min }\right)$.

\section{Clinical validation}

In total we included 21 sepsis patients, 15 cirrhosis patients, 7 females in their $3^{\text {rd }}$ trimester of pregnancy, and 20 cardiothoracic surgery (CTS) patients of which one patient did not receive tranexamic acid (this patient was omitted from all statistical analyses).

Complete blood count $(\mathrm{Hb}, \mathrm{Ht}, \mathrm{WBC}$, and PLT) and measurement of PT and aPTT was performed revealing results that are compatible for these patient groups (table 5.2). In figure 5.2 a comparison of the ROTEM profiles for each patient group to the group of healthy volunteers is displayed. As an example, the cardiothoracic surgery patient without having had tranexamic acid was added to this graph only. In the absence of rTPA, the CT was shorter for cirrhosis ( $p$ $=0.0121)$, equal for pregnancy $(p=0.5289)$, and longer for sepsis $(p<0.0001)$ and CTS $(p=0.0013)$ in comparison to healthy volunteers.

Table 5.2 Complete blood count of the healthy volunteers and patient groups

\begin{tabular}{|c|c|c|c|c|c|}
\hline & $\begin{array}{c}\text { Healthy } \\
\text { volunteers }\end{array}$ & Sepsis & CTS-TXA & $\begin{array}{c}\text { Cirrhotic liver } \\
\text { disease }\end{array}$ & Pregnancy \\
\hline $\mathrm{Hb}(\mathrm{mmol} / \mathrm{L})$ & $\begin{array}{c}9.0 \\
(8.4-9.6)\end{array}$ & $\begin{array}{c}6.0^{*} \\
(5.6-6.3)\end{array}$ & $\begin{array}{c}6.2^{*} \\
(5.7-6.7)\end{array}$ & $\begin{array}{c}7.7^{*} \\
(7.2-8.4)\end{array}$ & $\begin{array}{c}7.8^{*} \\
(7.0-8.4)\end{array}$ \\
\hline $\mathrm{Ht}(\mathrm{L} / \mathrm{L})$ & $\begin{array}{c}0.43 \\
(0.40-0.45)\end{array}$ & $\begin{array}{c}0.29^{*} \\
(0.27-0.31)\end{array}$ & $\begin{array}{l}0.29^{*} \\
(0.26- \\
0.32)\end{array}$ & $\begin{array}{c}0.38^{*} \\
(0.34-0.39)\end{array}$ & $\begin{array}{c}0.36^{*} \\
(0.34-0.39)\end{array}$ \\
\hline $\operatorname{PLT}\left(x 10^{9} / L\right)$ & $\begin{array}{c}178 \\
(139-212)\end{array}$ & $\begin{array}{c}141 \\
(95-213)\end{array}$ & $\begin{array}{c}101 \\
(91-140)\end{array}$ & $\begin{array}{c}99^{*} \\
(62-136)\end{array}$ & $\begin{array}{c}202 \\
(127-234)\end{array}$ \\
\hline WBC $\left(\times 10^{9} / L\right)$ & $\begin{array}{c}6.4 \\
(5.4-7.9)\end{array}$ & $\begin{array}{c}20.1^{*} \\
(11.5-25.3)\end{array}$ & $\begin{array}{c}10.7^{*} \\
(8.3-12.4)\end{array}$ & $\begin{array}{c}5.0 \\
(4.0-7.5)\end{array}$ & $\begin{array}{c}10.7^{*} \\
(9.3-11.7)\end{array}$ \\
\hline
\end{tabular}

${ }^{*} p<0.05$ in comparison to healthy volunteers

Results are median (IQR)

Abbreviations: Hb, haemoglobin; Ht, haematocrit; PLT, platelet count; WBC, white blood cell count; IQR, interquartile ranges; CTS-TXA; cardiothoracic surgery patients using tranexamic acid 

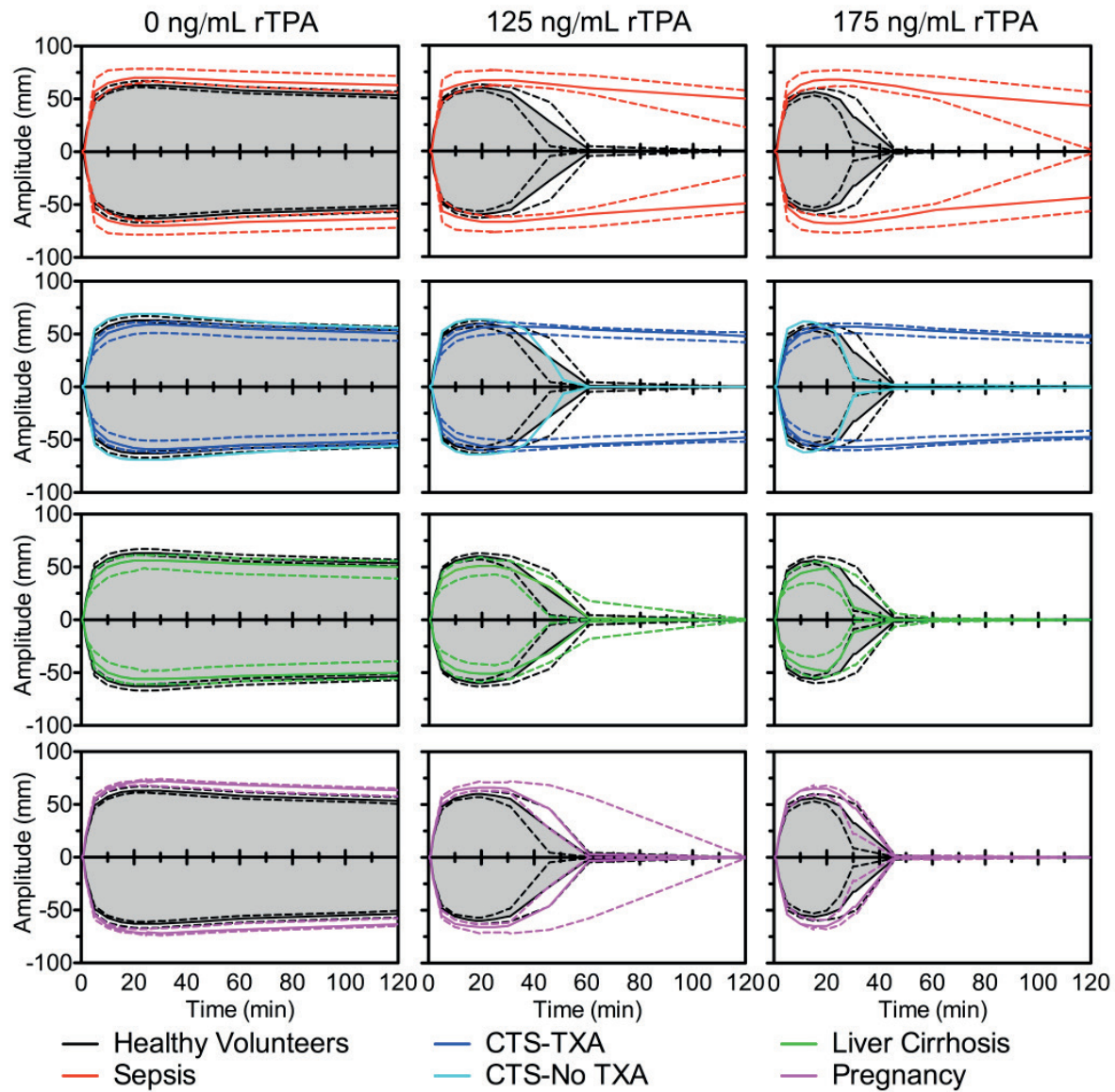

- CTS-TXA
CTS-No TXA

- Liver Cirrhosis

- Pregnancy

Figure 5.2 Normal and rTPA induced fibrinolysis profiles on a ROTEM device. Median tissue factor stimulated ROTEM traces (coloured solid lines) with different rTPA concentrations expressed as interquartile ranges (coloured dotted lines) of four patient groups compared to healthy volunteers (black and grey).

Abbreviations: CTS-TXA, cardiothoracic surgery patients using tranexamic acid; CTS-No TXA, cardiothoracic surgery patient not using tranexamic acid; rTPA, recombinant tissue plasminogen activator

Although all statistically significant, none of the median clotting times of the groups was outside the normal range established in healthy volunteers. The MCF was lower for CTS $(p=0.0001)$ and cirrhosis $(p=0.0014)$, while it was higher in sepsis $(p=0.0003)$ and pregnancy $(p=0.0008)$. The MCF in sepsis and pregnancy were both statistically as well as clinically significant in comparison to the healthy volunteers. LOT was prolonged in sepsis $(p=0.0041)$ and CTS $(p=0.0206)$, but was equal in cirrhosis $(p=0.1624)$ and pregnancy $(p=$ 
0.1279). The LT could not be determined in any of the groups within the runtime of $120 \mathrm{~min}$. At $125 \mathrm{ng} / \mathrm{mL}$ rTPA the LOT was prolonged in sepsis and CTS ( $p<$ 0.001 ), whereas the LOT was equal in cirrhosis and pregnancy. The same was seen for the $L T, F S$, and $F S_{c}$ results, noting that $L T$ could not be reached in any of the CTS patients as can be appreciated from figure 5.3.

In figure 5.4, boxplots are shown for $175 \mathrm{ng} / \mathrm{mL}$ rTPA for which the results were similar to the $125 \mathrm{ng} / \mathrm{mL}$ condition with LOT, LT, FS, and $\mathrm{FS}_{\mathrm{C}}$ prolonged in sepsis and CTS $(p<0.001)$ and equal in cirrhosis and pregnancy.

Lysis Onset Time $125 \mathrm{ng} / \mathrm{mL}$ rTPA

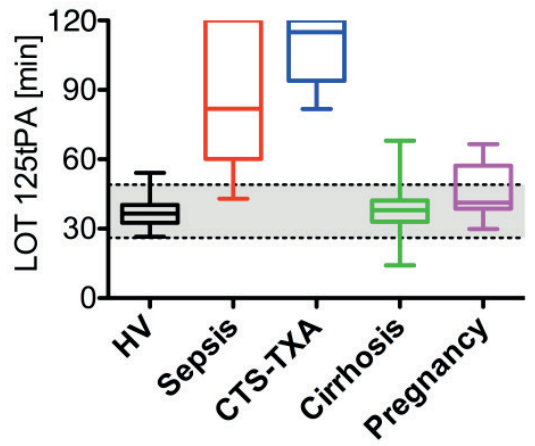

Fibrinolysis Speed $125 \mathrm{ng} / \mathrm{mL}$ rTPA

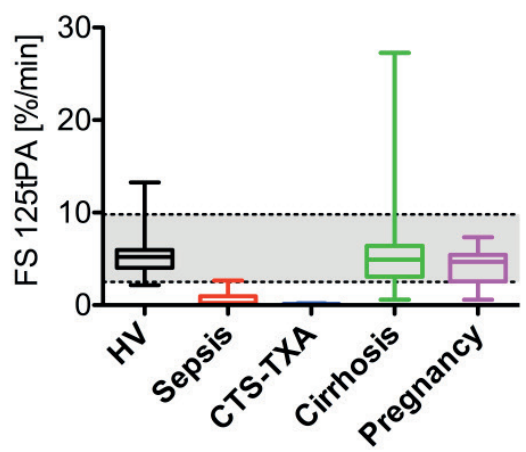

Lysis Time $125 \mathrm{ng} / \mathrm{mL}$ rTPA

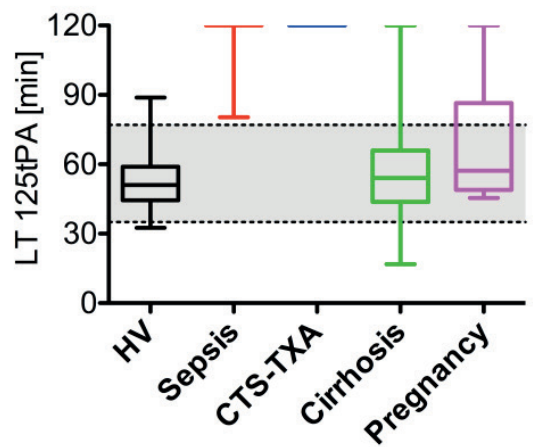

Corrected Fibrinolysis Speed $125 \mathrm{ng} / \mathrm{mL}$ rTPA

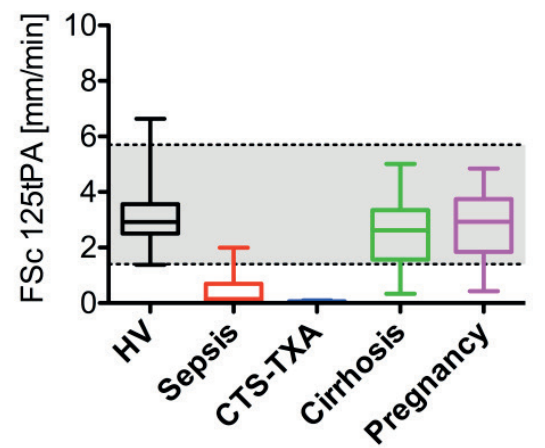

Figure 5.3 Boxplots of different ROTEM parameters at $125 \mathrm{ng} / \mathrm{mL}$ rTPA in healthy volunteers and patient groups. Sepsis and CTS are significantly different to HV $(p<0.001)$ for all four parameters. The grey area depicts the $95 \%$ reference ranges as was calculated using the healthy volunteer data from table 5.1.

Abbreviations: CTS-TXA, cardiothoracic surgery patients using tranexamic acid; $\mathrm{HV}$, healthy volunteers; rTPA, recombinant tissue plasminogen activator; CT, clotting time; MCF, maximum clot firmness; LOT, lysis onset time; LT, lysis time; FS, fibrinolysis speed, defined as the decline in \%/min between the LOT and LT points thus: $F S=75 /\left(\mathrm{LT}_{\min }-\mathrm{LOT} \min \right) ; \mathrm{FS}_{c}$, corrected fibrinolysis speed in $\mathrm{mm} / \mathrm{min}$ between the LOT and LT points thus: $\mathrm{FS}_{\mathrm{c}}=\left(\right.$ amplitude $\mathrm{LOT}_{\mathrm{LT}}-$ amplitude $\left._{\mathrm{LT}}\right) /\left(\mathrm{LT}_{\min }-\mathrm{LOT}_{\min }\right)$ 
Determinants of the cloth strength and fibrinolytic system were assessed for each patient group and the healthy volunteers and are presented in figure 5.5. Median D-dimer levels were elevated (>500 $\mathrm{ng} / \mathrm{L}$ ) in all patient groups, while median FXIII activity levels were below the reference ranges in sepsis (68.1\%) and CTS (62.3\%) only. Median plasminogen activity levels were reduced in sepsis $(81 \%)$ and especially in CTS (54\%) and cirrhosis (68\%), while being elevated in a pregnant state $(178 \%)$ when compared to healthy volunteers $(119 \%)$. The alpha 2-antiplasmin levels were equal in pregnancy $(117 \%)$ and

Lysis Onset Time $175 \mathrm{ng} / \mathrm{mL}$ rTPA

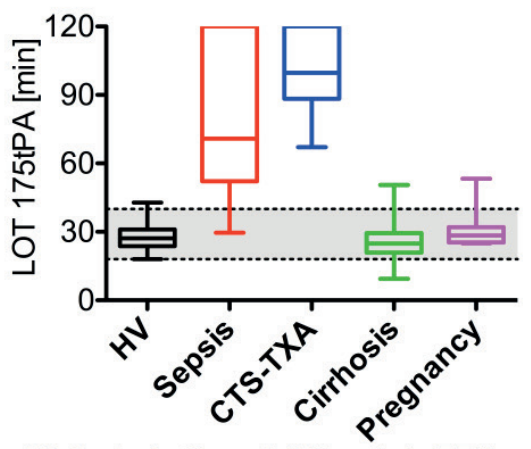

Fibrinolysis Speed $175 \mathrm{ng} / \mathrm{mL}$ rTPA

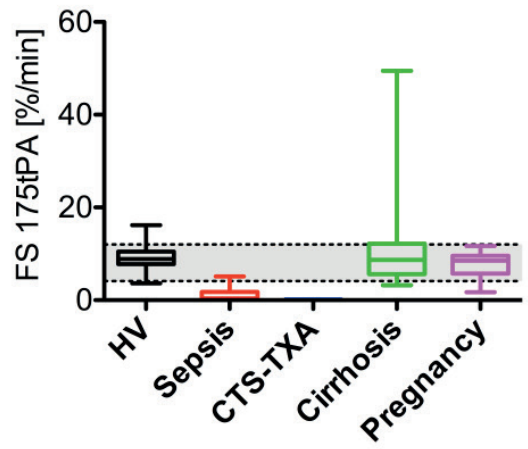

Lysis Time $175 \mathrm{ng} / \mathrm{mL}$ rTPA

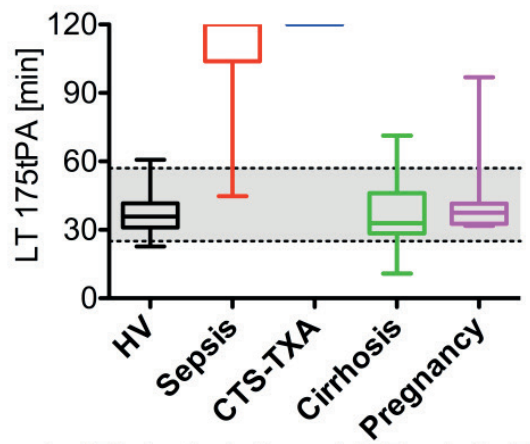

Corrected Fibrinolysis Speed $175 \mathrm{ng} / \mathrm{mL}$ rTPA

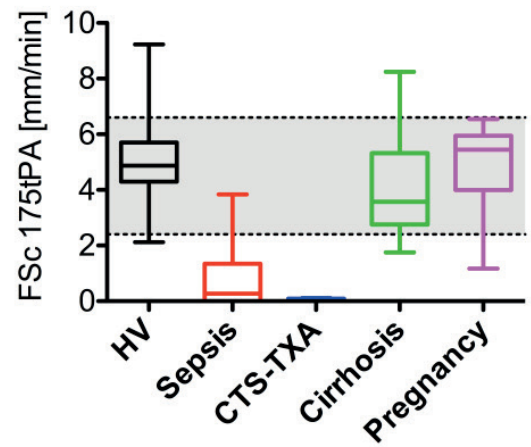

Figure 5.4 Boxplots of different ROTEM parameters at $175 \mathrm{ng} / \mathrm{mL}$ rTPA in healthy volunteers and patient groups. Sepsis and CTS are significantly different to HV $(p<0.001)$ for all four parameters. The grey area depicts the $95 \%$ reference ranges as was calculated using the healthy volunteer data from table 5.1 .

Abbreviations: CTS-TXA, cardiothoracic surgery patients using tranexamic acid; $\mathrm{HV}$, healthy volunteers; rTPA, recombinant tissue plasminogen activator; CT, clotting time; MCF, maximum clot firmness; LOT, lysis onset time; LT, lysis time; FS, fibrinolysis speed, defined as the decline in $\% / m i n$ between the LOT and LT points; thus: $F S=75 /\left(\mathrm{LT}_{\min }-\mathrm{LOT}_{\min }\right) ; \mathrm{FS}_{\mathrm{c}}$, corrected fibrinolysis speed in $\mathrm{mm} / \mathrm{min}$ between the LOT and LT points; thus: $\mathrm{FS}_{\mathrm{c}}=\left(\right.$ amplitude ${ }_{\mathrm{LOT}}-$ amplitude $\left._{\mathrm{LT}}\right) /\left(\mathrm{LT}_{\min }-\mathrm{LOT}_{\min }\right)$ 

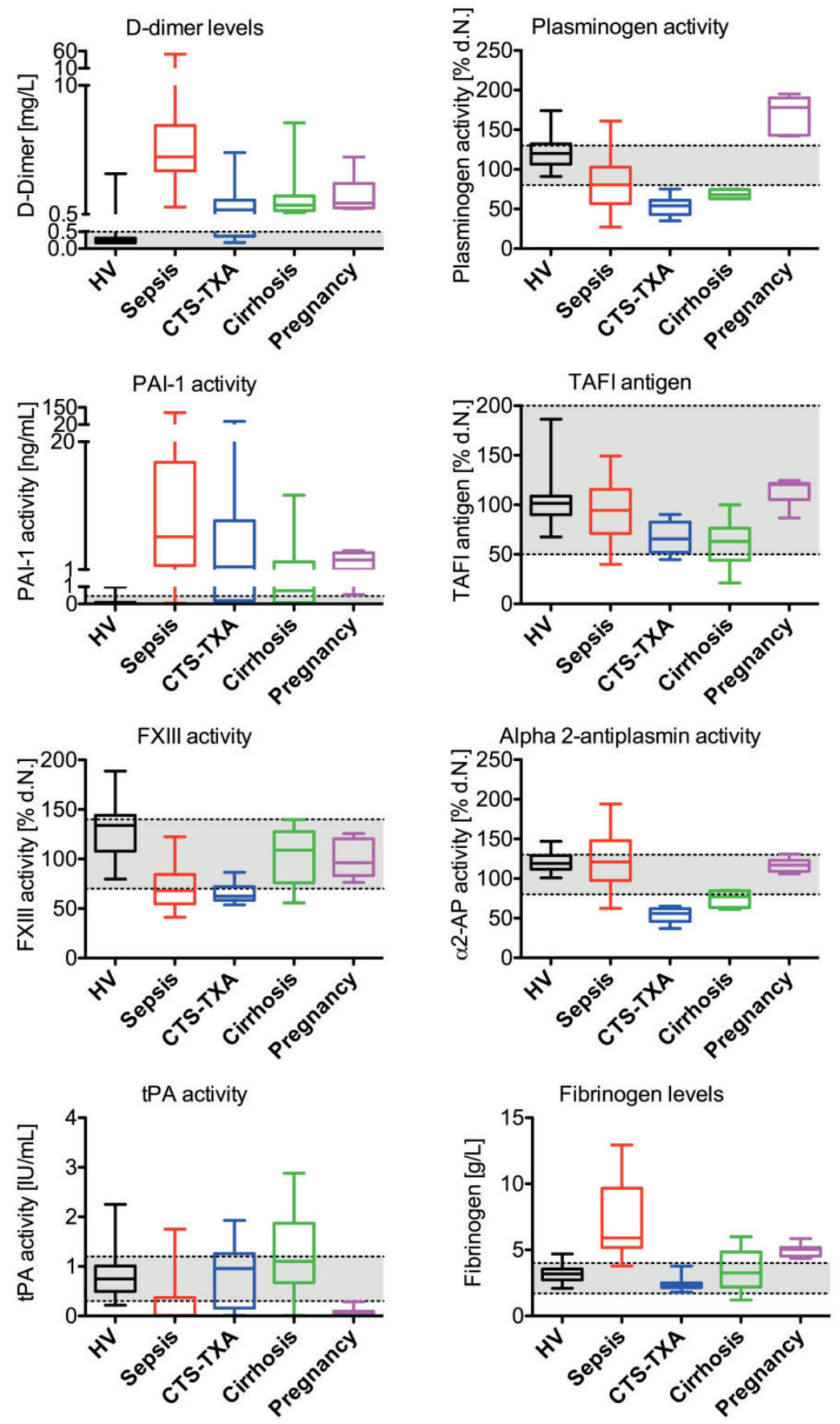

Figure 5.5 (See legend on next page.) 
Figure 5.5 (See figure on previous page.) Boxplots of the fibrinolysis determinants and net effect (D-dimer) respectively, in healthy volunteers and patient groups. The grey areas depict the in house established reference range of fibrinogen and D-dimer levels, while they represent the reference ranges according to the insert of the manufacturer in the four other parameters.

Abbreviations: CTS-TXA, cardiothoracic surgery patients using tranexamic acid; $\mathrm{HV}$, healthy volunteers; rTPA, recombinant tissue plasminogen activator; PAI-1, plasminogen activator inhibitor; TAFI, thrombin activated fibrinolysis inhibitor

sepsis $(121 \%)$ compared to healthy volunteers $(119 \%)$, but reduced in CTS $(56 \%)$ and cirrhosis (77\%). PAl-1 activity levels were high in all patient groups. In those with sepsis the median PAI-1 level was $6.0 \mathrm{ng} / \mathrm{mL}$ (IQR 1.7-17.0) and in those with pregnancy the median level was $2.5 \mathrm{ng} / \mathrm{mL}$ (IQR 1.1-3.6). tPA activity was low in sepsis $(0.01 \mathrm{IU} / \mathrm{mL})$ and pregnancy $(0.07 \mathrm{IU} / \mathrm{mL})$ and normal in CTS $(0.96 \mathrm{IU} / \mathrm{mL})$ and cirrhosis $(1.10 \mathrm{IU} / \mathrm{mL})$ patients. Median TAFI antigen levels were within normal range (50-200\%) in all groups. Fibrinogen, as more a procoagulant marker, together with platelet levels both account for the MCF in the $0 \mathrm{ng} / \mathrm{mL}$ rTPA condition with a significant Pearson correlation $r$ of 0.71 and 0.57 , respectively.

ECLT's (shown in figure 5.6) in healthy volunteers revealed a median ECLT of $95 \mathrm{~min}$ (IQR 76-114 min). In sepsis 67\% had ECLT's $\geq 4 \mathrm{~h}, 19 \% \geq 2 \mathrm{~h}$ and $14 \%$ $\leq 2 \mathrm{~h}$. All ECLT's in the pregnant group were $\geq 2 \mathrm{~h}$. In cardiothoracic surgery $80 \%$ had ECLT shorter than $2 \mathrm{~h}$ with a median of 85 min (IQR 71-117). In cirrhosis, only $13 \%$ had $E C L T \geq 2 h$. All the others had a median of 50 min (IQR 30-95).

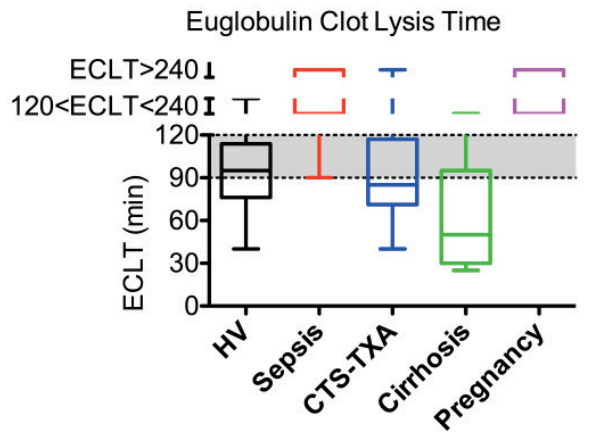

Figure 5.6 Boxplots of euglobulin clot lysis times in healthy volunteers and patient groups. The grey areas depict the reference range of euglobulin clot lysis time.

Abbreviations: CTS-TXA, cardiothoracic surgery patients using tranexamic acid; ECLT, euglobulin clot lysis time; HV, healthy volunteers 


\section{Discussion}

We established a protocol for the ROTEM-based rTPA induced fibrinolysis assay by defining the final concentrations for $\mathrm{TF}, \mathrm{CaCl}_{2}$ and rTPA using whole blood of healthy volunteers and validated this assay, to investigate, in patient cohorts their different fibrinolytic states. The baseline assay, applying only TF and $\mathrm{CaCl}_{2}$ with no rTPA in whole blood, showed good reproducibility with low $\mathrm{CV}$ percentages similar to those reported for the commercial EXTEM test, ${ }^{25}$ with the exception of higher $\mathrm{CV}$ percentages for $\mathrm{CT}$ values, which can be attributed to the manual pipetting process used in our protocol. Addition of rTPA resulted in higher CV percentages for lysis parameters, but these were acceptable at values below $20 \%$. Besides, the final concentrations of rTPA of 125 and 175 $\mathrm{ng} / \mathrm{mL}$ gave practical runtimes below $60 \mathrm{~min}$ in healthy volunteers, which is advantageous over plasma based fibrinolysis tests which include centrifugation steps. One other finding is the prolongation of the CT and attenuation of the MCF by the addition of rTPA, which is possibly due to the earlier start of fibrinolysis while the clot is being still formed. The need for manual pipetting introduced some possible artefacts in the CT results for this study. These results are therefore operator dependable. For example, we found that the CT results in male were shorter in comparison to female volunteers. This is in contrast to other research, ${ }^{26}$ but could be explained by the fact that manual pipetting was used.

When comparing to previous research, different concentrations of rTPA were chosen ranging from $50 \mathrm{ng} / \mathrm{ml}^{10}$ to $100 \mathrm{ng} / \mathrm{ml}^{11}$ and from $100 \mathrm{U} / \mathrm{ml}^{21}$ to 1000 $\mathrm{U} / \mathrm{ml}^{20}$ depending on the needs for the test. The combination of a "low" and a "high" rTPA concentration would provide suitable test conditions to reflect changes in a fibrinolysis balance that might be tilted towards hypo- or hyperfibrinolysis. One could argue that the $125 \mathrm{ng} / \mathrm{mL}$ rTPA concentration may be too high already for diagnosing a hyperfibrinolytical state and that too much contrast is lost. Choosing a lower concentration of rTPA, however, resulted in longer runtimes (data not shown), hence $125 \mathrm{ng} / \mathrm{mL}$ rTPA together with a runtime of 60 min seemed optimal in normal individuals. The $175 \mathrm{ng} / \mathrm{ml}$ rTPA concentration 
was chosen as it diminished the lysis time by $25 \%$ in normal healthy individuals. However, in our patient subsets still little effects were seen in sepsis and after cardiothoracic surgery at $175 \mathrm{ng} / \mathrm{ml} \mathrm{rTPA}$, however this concentration seems reasonable for diagnosing hypofibrinolysis. The $125 \mathrm{ng} / \mathrm{ml}$ concentration is proven to be too high for hyperfibrinolytical states. A lower concentration could improve hyperfibrinolysis testing by sacrificing short runtimes. One point which needs to be made is that for TEG (trombelastography) analysis, the use of a native TEG (non-activated) versus a RapidTEG (kaolin activated) seems to influence fibrinolysis results. ${ }^{27}$ Whether this is also the case for our rTPA induced tissue factor activated ROTEM analysis is unclear.

When determining the effects of processing time from blood sampling to ROTEM analysis, there appears to be little effect on test outcomes within a $4 \mathrm{~h}$ period, which is reasonable for clinical purposes. The 95\% reference intervals were assessed using whole blood from 40 healthy volunteers. We decided however to normalize the results and calculate 95\% intervals and then transforming them back, making the 95\% reference intervals smaller than advised in order to correct for possible outliers. ${ }^{28}$ The reference intervals in our $0 \mathrm{ng} / \mathrm{mL}$ rTPA for CT and MCF are in line with the commercial reagents for the EXTEM test. The reference intervals for the commercial EXTEM CT and MCF are 36-60 $\mathrm{s}$ and 48-72 $\mathrm{mm}$ respectively, as were established in our hospital healthy volunteer population. Reference intervals for LOT and LT using the EXTEM test were never established, as these seem irrelevant in a healthy population. LOT, LT, and other lysis index parameters (LI30, LI45, LI60) are used in clinical practice in overt hyperfibrinolysis. For the clinical validation we selected patients, which according to literature could have a hyper- or hypofibrinolytic potential and compared them to healthy volunteers. The need for an rTPA induced method of assessing fibrinolysis in the ROTEM assay becomes obvious when comparing test conditions with rTPA versus those without rTPA addition. Within a time span of $2 \mathrm{~h}$ no distinction can be made between any of the groups and healthy population with regard to fibrinolytic potential, either visually or by using CT, MCF, LOT, or LT parameters in the absence of added rTPA. As illustrated in figure 5.2, marked differences between healthy persons on the one hand and 


\section{Chapter 5}

different patient populations on the other hand are detectable with added rTPA. In subjects with sepsis but without an overt state of DIC (according to ISTH criteria) a hypofibrinolytic state (long LOT and LT combined with reduced FS and $\mathrm{FS}_{\mathrm{c}}$ ) in conjunction with a marked procoagulant state (high MCF) is evident, despite the fact that high D-dimer levels suggest a high fibrinolytic activity. ECLT testing showed a hypofibrinolytic state in sepsis, which is in agreement with our rTPA induced ROTEM assay. D-dimer levels are determinants of different processes, ${ }^{29}$ including the generation of fibrin as well as the rather nonspecific cleavage of polymerized fibrin molecules by various enzymes including plasmin but also elastase, trypsin and others that are released during sepsis by neutrophils and other inflammatory cells. The high PAI-1 activity is thought to be primarily responsible for the attenuated fibrinolysis in patients with sepsis. Transient elevation of TPA and PAI-1 exists due to endothelial damage, but soon after, IPA antigen and activity is lowered through inactivation by increasing levels of PAI- $1 .^{30}$ Theoretically, upon progression of sepsis and occurrence of overt DIC alpha 2-antiplasmin would be consumed and the ROTEM pattern may change. This was not found in the early sepsis group and we did not address conversion to DIC in the present investigation. In patients with CTS being administered tranexamic acid a hypofibrinolytic state is observed (reduced FS and $\mathrm{FS}_{\mathrm{c}}$ ), although an overall reduced maximum clot firmness to start with is present in this group. The ECLT in this group did not show a hypofibrinolytic state, but rather a normal state when compared to healthy volunteers. The difference between the ECLT and our ROTEM assay appears to be due to the fact that tranexamic acid does not precipitate in the ECLT test. ${ }^{31}$ Reduced MCF is seen following dilutional effects during ECC by lowering fibrinogen levels and platelet numbers. Furthermore, consumption of fibrinogen during cardiac surgery and the subsequent raised D-dimer levels are seen in our study. PAI-1 levels are high in our study for CTS, which, next to tranexamic acid, could strengthen clot firmness and prevent excessive breakdown by fibrinolytic enzymes (which could be reflected by the reduced activity of plasminogen) and could counteract the reduced antifibrinolytic activity of alpha 2-antiplasmin. Reduced activity of FXIIla together with a hypofibrinolytic state is seen in CTS, ${ }^{32}$ which could be the result 
of FXIII consumption and dilution. The additional effect of tranexamic acid resulted in a hypofibrinolytical profile in our ROTEM assay, while in the one patient not having had tranexamic acid it showed a normal fibrinolytic profile compared to healthy volunteers. Tranexamic acid counteracts the effects of ECC induced endogenous tPA release, caused by shear stress on the vessel wall ${ }^{33}$ and haemodilutional effects of cardiothoracic surgery, ${ }^{34}$ and may stabilize clots even more than needed. Cirrhotic liver disease patients do not seem to differ from healthy volunteers regarding their global fibrinolytic rTPA-ROTEM profile with equal LOT, LT, FS, and $\mathrm{FS}_{\mathrm{C}}$ in both rTPA induced conditions together with a globally reduced procoagulant state. The ECLT was markedly reduced. Both findings are coherent with other studies as was researched and discussed previously by our group. ${ }^{35}$ This reset balance in haemostasis is characterized by a reduced level of both the procoagulant (e.g. fibrinogen) and the antifibrinolytic factors (e.g. low alpha 2-antiplasmin). ${ }^{36}$ tPA is thought to be increasing over time, while PAI-1 activity levels are decreasing. ${ }^{37}$ This could theoretically result in more pronounced hyperfibrinolysis. High D-dimer levels indicate ongoing lysis. Again, high D-dimer levels are seen in almost any thrombotic disease state. Some individuals with cirrhosis showed very high fibrinolysis speeds (FS), but when these are corrected for their already reduced MCF, the corrected fibrinolysis speed $\left(F S_{c}\right)$ might give a more accurate view on fibrinolysis potential. The use of the $\mathrm{FS}_{\mathrm{c}}$ seem to be more helpful when interpreting fibrinolysis potentials instead of the FS in assessing the haemostatic balance by correcting for fibrinogen and platelet counts which are the main correlates for MCF. Again the $125 \mathrm{ng} / \mathrm{ml}$ rTPA concentration might be too high in patients with hyperfibrinolysis activity and a lower concentration of rTPA could reveal more pronounced hyperfibrinolysis in cirrhosis. Pregnancy is associated with a gradual increase in PAl-1 over time, ${ }^{38}$ as confirmed in our study. TAFI and fibrinogen levels are also higher in comparison to healthy volunteers. ${ }^{39-41}$ tPA activity levels were lower in comparison to the healthy volunteers, which is comparable to previous studies. ${ }^{41}$ An incline in tPA levels was shown followed by a drop in tPA levels postpartum. In our study, reduced tPA levels could result in higher than normal plasminogen activity. ROTEM reference values in pregnancy were established 


\section{Chapter 5}

previously in a study from our clinics and are in line with the present findings. ${ }^{42}$ Hypofibrinolysis on our ROTEM assay could not be detected, but was clearly present in the ECLT test. Fibrinogen levels and also platelet levels seem to correlate well with MCF for any given group, an all-by-all no new finding. ${ }^{43}$ Thus far the test is able to pick up hypofibrinolysis accurately in groups of patients with the $175 \mathrm{ng} / \mathrm{mL}$ rTPA concentration and could guide therapy in CTS and sepsis. One could argue that the amount of tranexamic acid given to CTS patients is too much if even at the high concentration of rTPA no fibrinolysis is seen within $2 \mathrm{~h}$. This assay could be used to titrate the drug following the ROTEM graphs and results in conjunction with clinical relevant outcomes. Trauma induced coagulopathy (TIC) is a very interesting syndrome in which overt hyperfbrinolysis is sometimes seen using ROTEM without the need for rTPA addition. As is shown by the CRASH-2 trial $^{8}$ the use of tranexamic acid reduces mortality when given within $3 \mathrm{~h}$ of the trauma. In our study a trauma population could not be assessed because of low numbers of severe trauma patients seen in our hospital during normal operating hours. Much research is done in this study population using TEG technology. ${ }^{12}$ ROTEM and TEG are essentially the same techniques, but differ slightly in measuring principle, reagents used, and naming conventions. ${ }^{43}$ In a recent paper by Moore et al ${ }^{44}$ hyperfibrinolysis seen on non-rTPA induced TEG analysis was associated with higher mortality in comparison to a physiological subgroup, while shutdown of hyperfibrinolysis was associated with lower mortality rates in comparison to hyperfibrinolysis, but these still being higher than the physiological subgroup. This resulted in a U-shaped optimum curve in which careful titration of tranexamic acid could result in better survival rates. High mortality rate due to fibrinolysis shutdown was also found by Raza et al. ${ }^{1}$ A recent review by Dobson et al ${ }^{45}$ discusses the timeline in trauma induced coagulopathy and the review by Napolitano et $\mathrm{al}^{46}$ extensively researched the literature to make recommendations regarding tranexamic acid use in trauma. For this purpose implementation of our rTPA-ROTEM assay may shed more light on this subject matter. As a limitation, hyperfibrinolysis could not be shown in a group of patients with cirrhosis when averaging their results. However, some individual patients do show a 
hyperfibrinolytic potential, while others do not. Still, this assay might be useful for individual patients with cirrhotic liver disease to diagnose (and treat) hyperfibrinolysis, although the rTPA addition needs titrating to a lower concentration. Another limitation is the manual pipetting that is required up till now. Having ready to use solutions and implementing them together with the automatic pipetting function on the ROTEM device could greatly reduce the inter-observer variability, especially for the clotting times. Our rTPA induced fibrinolysis ROTEM assay showed different results in comparison to the ECLT test. This is because there are essential differences between both tests. The ECLT is plasma based and due to acidification of the plasma only few factors are precipitated, the euglobulin fraction. As such, the role of red blood cells and thrombocytes is excluded in the ECLT on fibrinolysis regulation. This makes our test a valuable addition to the arsenal of fibrinolysis testing.

\section{Conclusions}

In conclusion, we developed a reproducible whole blood rTPA ROTEM assay for diagnosing hypofibrinolytic potential with short turnaround times, which in healthy volunteers seems to produce acceptable variations resulting in narrow reference intervals. The $175 \mathrm{ng} / \mathrm{ml}$ rTPA concentration appears to be sensitive for the medication effect of tranexamic acid on fibrinolysis, which is in contrast to the ECLT for tranexamic acid. This $175 \mathrm{ng} / \mathrm{ml}$ concentration may distinguish a hypo- from a normofibrinolytic potential. The implementation of this test needs to be evaluated in more prominent hyperfibrinolytic disease states, like trauma or thrombolysis therapy, in order to address the potential utility in the diagnostic workup of patients with fibrinolytic dysfunction, as well as in guiding antifibrinolytic management. 


\section{Chapter 5}

\section{References}

1. Raza I, Davenport R, Rourke C, Platton S, Manson J, Spoors C, Khan S, De'Ath HD, Allard S, Hart DP, Pasi KJ, Hunt BJ, Stanworth S, MacCallum PK, Brohi K. The incidence and magnitude of fibrinolytic activation in trauma patients. J Thromb Haemost. 2013 Feb;11(2):307-14.

2. Rijken DC, Kock EL, Guimarães AH, Talens S, Darwish Murad S, Janssen HL, Leebeek FW. Evidence for an enhanced fibrinolytic capacity in cirrhosis as measured with two different global fibrinolysis tests. J Thromb Haemost. 2012 Oct;10(10):2116-22.

3. Smith AA, Jacobson LJ, Miller BI, Hathaway WE, Manco-Johnson MJ. A new euglobulin clot lysis assay for global fibrinolysis. Thromb Res. 2003;112(5-6):329-37.

4. Wada H. Disseminated intravascular coagulation. Clin Chim Acta. 2004 Jun;344(1-2):13-21.

5. Ten Cate H. Pathophysiology of disseminated intravascular coagulation in sepsis. Crit Care Med. 2000 Sep;28(9 Suppl):S9-11.

6. Zeerleder S, Schroeder V, Lämmle B, Wuillemin WA, Hack CE, Kohler HP. Factor XIII in severe sepsis and septic shock. Thromb Res. 2007;119(3):311-8.

7. McCormack PL. Tranexamic acid: a review of its use in the treatment of hyperfibrinolysis. Drugs. 2012 Mar 26;72(5):585-617.

8. The CRASH-2 collaborators. The importance of early treatment with tranexamic acid in bleeding trauma patients: an exploratory analysis of the CRASH-2 randomised controlled trial. Lancet. 2011 Mar 26;377(9771):1096-101, 1101.e1-2.

9. Chitlur M. Challenges in the laboratory analyses of bleeding disorders. Thromb Res. 2012 Jul;130(1):1-6.

10. Kupesiz A, Rajpurkar M, Warrier I, Hollon W, Tosun O, Lusher J, Chitlur M. Tissue plasminogen activator induced fibrinolysis: standardization of method using thromboelastography. Blood Coagul Fibrinolysis. 2010 Jun;21(4):320-4.

11. Dirkmann D, Radü-Berlemann J, Görlinger K, Peters J. Recombinant tissue-type plasminogen activator-evoked hyperfibrinolysis is enhanced by acidosis and inhibited by hypothermia but still can be blocked by tranexamic acid. J Trauma Acute Care Surg. 2013 Feb;74(2):482-8.

12. Moore HB, Moore EE, Chapman MP, Gonzalez E, Slaughter AL, Morton AP, D'Alessandro A, Hansen KC, Sauaia A, Banerjee A, Silliman CC. Viscoelastic measurements of platelet function, not fibrinogen function, predicts sensitivity to tissue-type plasminogen activator in trauma patients. J Thromb Haemost. 2015 Oct;13(10):1878-87.

13. Gallimore MJ, Harris SL, Tappenden KA, Winter M, Jones DW. Urokinase induced fibrinolysis in thromboelastography: a model for studying fibrinolysis and coagulation in whole blood. J Thromb Haemost. 2005 Nov;3(11):2506-13.

14. Dargaud Y, Prevost C, Lienhart A, Claude Bordet J, Negrier C. Evaluation of the overall haemostatic effect of recombinant factor VIla by measuring thrombin generation and stability of fibrin clots. Haemophilia. 2011 Nov;17(6):957-61.

15. Franz RC. ROTEM analysis: a significant advance in the field of rotational thrombelastography. S Afr J Surg. 2009 Feb;47(1):2-6. 
16. Mittermayr M, Streif W, Haas T, Fries D, Velik-Salchner C, Klingler A, Innerhofer P. Effects of colloid and crystalloid solutions on endogenous activation of fibrinolysis and resistance of polymerized fibrin to recombinant tissue plasminogen activator added ex vivo. $\mathrm{Br} \mathrm{J}$ Anaesth. 2008 Mar;100(3):307-14.

17. Katori N, Tanaka KA, Szlam F, Levy JH. The effects of platelet count on clot retraction and tissue plasminogen activator-induced fibrinolysis on thrombelastography. Anesth Analg. 2005 Jun;100(6):1781-5.

18. Shenkman B, Livnat T, Budnik I, Tamarin I, Einav Y, Martinowitz U. Plasma tissue-type plasminogen activator increases fibrinolytic activity of exogenous urokinase-type plasminogen activator. Blood Coagul Fibrinolysis. 2012 Dec;23(8):729-33.

19. Viuff D, Andersen S, Sørensen BB, Lethagen S. Optimizing thrombelastography (TEG) assay conditions to monitor rFVIIa (NovoSeven) therapy in haemophilia A patients. Thromb Res. 2010 Aug;126(2):144-9.

20. Nielsen VG, Cankovic L, Steenwyk BL. Epsilon-aminocaproic acid inhibition of fibrinolysis in vitro: should the 'therapeutic' concentration be reconsidered? Blood Coagul Fibrinolysis. 2007 Jan;18(1):35-9.

21. Nielsen VG, Cohen BM, Cohen E. Elastic modulus-based thrombelastographic quantification of plasma clot fibrinolysis with progressive plasminogen activation. Blood Coagul Fibrinolysis. 2006 Jan;17(1):75-81.

22. Annane D, Bellissant E, Cavaillon JM. Septic shock. Lancet. 2005 Jan 1-7;365(9453):63-78.

23. Toh $\mathrm{CH}$, Downey C. Performance and prognostic importance of a new clinical and laboratory scoring system for identifying non-overt disseminated intravascular coagulation. Blood Coagul Fibrinolysis. 2005 Jan;16(1):69-74.

24. Kowalski E, Kopeć M. Niewiarowski S. An evaluation of the euglobulin method for the determination of fibrinolysis. J Clin Pathol. 1959 May;12(3):215-8.

25. Kitchen DP, Kitchen S, Jennings I, Woods T, Walker I. Quality assurance and quality control of thrombelastography and rotational Thromboelastometry: the UK NEQAS for blood coagulation experience. Semin Thromb Hemost. 2010 Oct;36(7):757-63.

26. Sucker C, Tharra K, Litmathe J, Scharf RE, Zotz RB. Rotation thromboelastography (ROTEM) parameters are influenced by age, gender, and oral contraception. Perfusion. 2011 Jul;26(4):334-40.

27. Thai J, Reynolds EJ, Natalia N, Cornelissen C, Lemmens HJ, Hill CC, van der Starre PJ. Comparison between RapidTEG $\AA$ and conventional thromboelastography in cardiac surgery patients. Br J Anaesth. 2011 Apr;106(4):605-6.

28. Horn PS, Pesce AJ. Reference intervals: an update. Clin Chim Acta. 2003 Aug;334(1-2):5-23.

29. Kabrhel C, Mark Courtney D, Camargo CA Jr, Plewa MC, Nordenholz KE, Moore CL, Richman PB, Smithline HA, Beam DM, Kline JA. Factors associated with positive D-dimer results in patients evaluated for pulmonary embolism. Acad Emerg Med. 2010 Jun;17(6):589-97.

30. Gando S. Role of fibrinolysis in sepsis. Semin Thromb Hemost. 2013 Jun;39(4):392-9. 


\section{Chapter 5}

31. Katz J, Lurie A, Becker D, Metz J. The euglobulin lysis time test: an ineffectual monitor of the therapeutic inhibition of fibrinolysis. J Clin Pathol. 1970 Sep;23(6):529-32.

32. Chandler WL, Patel MA, Gravelle L, Soltow LO, Lewis K, Bishop PD, Spiess BD. Factor XIIIA and clot strength after cardiopulmonary bypass. Blood Coagul Fibrinolysis. 2001 Mar;12(2):1018.

33. Yavari M, Becker RC. Coagulation and fibrinolytic protein kinetics in cardiopulmonary bypass. J Thromb Thrombolysis. 2009 Jan;27(1):95-104.

34. Woodman RC, Harker LA. Bleeding complications associated with cardiopulmonary bypass. Blood. 1990 Nov 1;76(9):1680-97.

35. Kleinegris MC, Bos MH, Roest M, Henskens Y, Ten Cate-Hoek A, Van Deursen C, Spronk HM, Reitsma PH, De Groot PG, Ten Cate H, Koek G. Cirrhosis patients have a coagulopathy that is associated with decreased clot formation capacity. J Thromb Haemost. 2014 Oct;12(10):164757.

36. Tripodi A, Mannucci PM. The coagulopathy of chronic liver disease. N Engl J Med. 2011 Jul 14;365(2):147-56.

37. Ferguson JW, Helmy A, Ludlam C, Webb DJ, Hayes PC, Newby DC. Hyperfibrinolysis in alcoholic cirrhosis: relative plasminogen activator inhibitor type 1 deficiency. Thromb Res. 2008;121(5):675-80.

38. Robb AO, Mills NL, Din JN, Cameron S, Ludlam CA, Newby DE, Denison FC. Acute endothelial tissue plasminogen activator release in pregnancy. J Thromb Haemost. 2009 Jan;7(1):138-42.

39. Knol HM, Veeger NJ, Middeldorp S, Hamulyák K, Van Der Meer J. High thrombin-activatable fibrinolysis inhibitor levels may protect against recurrent fetal loss. J Thromb Haemost. 2009 May;7(5):903-6.

40. Mousa HA, Downey C, Alfirevic Z, Toh CH. Thrombin activatable fibrinolysis inhibitor and its fibrinolytic effect in normal pregnancy. Thromb Haemost. 2004 Nov;92(5):1025-31.

41. Cerneca F, Ricci G, Simeone R, Malisano M, Alberico S, Guaschino S. Coagulation and fibrinolysis changes in normal pregnancy. Increased levels of procoagulants and reduced levels of inhibitors during pregnancy induce a hypercoagulable state, combined with a reactive fibrinolysis. Eur J Obstet Gynecol Reprod Biol. 1997 May;73(1):31-6.

42. De Lange NM, van Rheenen-Flach LE, Lancé MD, Mooyman L, Woiski M, van Pampus EC, Porath M, Bolte AC, Smits L, Henskens YM, Scheepers HC. Peri-partum reference ranges for ROTEM(R) thromboelastometry. Br J Anaesth. 2014 May;112(5):852-9.

43. Luddington RJ. Thrombelastography/thromboelastometry. Clin Lab Haematol. 2005 Apr;27(2):81-90.

44. Moore HB, Moore EE, Gonzalez E, Chapman MP, Chin TL, Silliman CC, Banerjee A, Sauaia A. Hyperfibrinolysis, physiologic fibrinolysis, and fibrinolysis shutdown: the spectrum of postinjury fibrinolysis and relevance to antifibrinolytic therapy. J Trauma Acute Care Surg. 2014 Dec;77(6):811-7. 
45. Dobson GP, Letson HL, Sharma R, Sheppard FR, Cap AP. Mechanisms of early traumainduced coagulopathy: The clot thickens or not? J Trauma Acute Care Surg. 2015 Aug;79(2):301-9.

46. Napolitano LM, Cohen MJ, Cotton BA, Schreiber MA, Moore EE. Tranexamic acid in trauma: how should we use it? J Trauma Acute Care Surg. 2013 Jun;74(6):1575-86. 



\section{Chapter 6}

\section{Rapid and correct prediction}

\section{of thrombocytopenia and} hypofibrinogenemia with

\section{rotational thromboelastometry}

in cardiac surgery

Olde Engberink RH, Kuiper GJ, Wetzels RJ, Nelemans PJ, Lancé MD, Beckers EA, Henskens YM

J Cardiothorac Vasc Anesth. 2014 Apr;28(2):210-6. 


\section{Abstract}

\section{Objectives}

In the present study, the authors have investigated whether rotational thromboelastometry (ROTEM) could predict thrombocytopenia and hypofibrinogenemia in cardiac surgery using the clot amplitude after 5 minutes (A5). Another parameter, PLTEM, in which the contribution of fibrinogen is eliminated by subtracting a fibrin-specific ROTEM test (FIBTEM) from an extrinsically-activated ROTEM test (EXTEM), was investigated. Furthermore, the turn-around time of ROTEM was compared to conventional laboratory tests.

\section{Design}

Prospective cohort study.

\section{Setting}

Single academic medical centre.

\section{Participants}

Ninety-seven patients undergoing cardiac surgery between July 2011 until August 2012.

\section{Interventions}

The correlations between EXTEM/FIBTEM A5, A10, and maximal clot formation (MCF), EXTEM/PLTEM (A5, A10, and MCF) and platelet count, and FIBTEM (A5, A10, and MCF) and fibrinogen were evaluated using the Pearson's correlation coefficient and receiver-operating characteristic curves. Turnaround times of ROTEM tests and conventional laboratory tests were assessed in the central laboratory.

\section{Measurements and Main Results}

EXTEM A5 and FIBTEM A5 showed an excellent correlation with A10 $(r=0.99 / 1.00)$ and MCF $(r=$ 0.97/0.99). The correlation between EXTEM A5 and platelet count $(r=0.74)$ was comparable with the correlation of A10 $(r=0.73)$ and MCF $(r=0.70)$ with platelet count. FIBTEM A5 predicted fibrinogen levels $(r=0.87)$ as well as A10 $(r=0.86)$ and MCF $(r=0.87)$. PLTEM A5 $(r=0.85)$ correlated better with platelet count than EXTEM A5 $(r=0.74 ; p=0.04)$ and showed significantly better area under the curve values than EXTEM for predicting thrombocytopenia (A5 $p=0.012$, A10 $p=0.019$ ). Turnaround time for ROTEM tests, 12 minutes, was comparable with emergency requests for platelet count, 13 minutes, and shorter than emergency requests for fibrinogen levels, 37 minutes.

\section{Conclusions}

Implementation of PLTEM and FIBTEM A5 in ROTEM-guided transfusion protocols may improve transfusion management. 


\section{Chapter 6}

\section{Introduction}

During cardiac surgery blood coagulation is subjected to many unfavourable changes due to haemodilution, loss of platelets, and deficiencies of both coagulation factors and inhibitors. ${ }^{1,2}$ Under such circumstances, rapid assessment of haemostatic function is necessary to guide decisions on optimal transfusion management. Conventional laboratory tests, although often used, are not very helpful to direct transfusion management in these urgent situations due to their long turnaround time. In emergency cases, blood transfusions or therapy with isolated coagulation factor concentrates (e.g. fibrinogen) are, therefore, often performed empirically before laboratory results are available. This could result in suboptimal treatment.

Because of its short turnaround time, rotational thromboelastometry (ROTEM) has proven to be an important diagnostic tool in situations of massive blood loss, such as cardiac and hepatic surgery and major trauma. ${ }^{3-8}$ In both cardiac and non-cardiac surgery, an extrinsically-activated ROTEM test (EXTEM) and a fibrin-specific ROTEM test (FIBTEM) have been shown to provide information about platelet count and fibrinogen levels within 10 to 20 minutes. ${ }^{5}$ The use of these ROTEM parameters in transfusion protocols has been reported to reduce the amount of blood loss and the incidence of blood transfusion. ${ }^{9-11}$ Although multiple guidelines recommend the use of conventional laboratory tests in situations of major blood loss, it generally is agreed that thromboelastometry could be an important diagnostic tool to assist in guiding haemostatic therapy and characterizing the coagulopathy. ${ }^{12,13}$

In this study, the authors have prospectively collected data to determine whether ROTEM could predict thrombocytopenia and hypofibrinogenemia in cardiac surgery using the amplitude after 5 minutes (A5). They hypothesized that A5 could estimate platelet count and fibrinogen levels as well as A10 and the maximal clot formation (MCF), which currently are used in ROTEM-guided transfusion protocols. Since limited information about turnaround times for convention- 


\section{Chapter 6}

al tests for platelet count and fibrinogen levels have been reported in the literature,$^{14}$ the turnaround times of conventional tests were compared with ROTEM tests to quantify the time advantage of A5 in the clinical setting of cardiac surgery. In addition, the authors calculated another ROTEM variable (PLTEM) by subtracting FIBTEM from EXTEM. They hypothesized that PLTEM could estimate platelet count more accurately than EXTEM.

\section{Materials and methods}

This study was part of a planned evaluation after the introduction of ROTEM as a diagnostic tool during cardiac surgery in a single university hospital. From July 2011 until August 2012, all patients with ROTEM results, both during and after cardiopulmonary bypass (CPB), were included. ROTEM tests were not performed on fixed time points. Indications for ROTEM tests were an increased preoperative bleeding risk, rethoracotomy, combined surgery, aorta surgery, active endocarditis, deep hypothermic circulatory arrest, emergency surgery, CPB time more than 180 minutes, excessive blood loss after protamine administration, more than $200 \mathrm{~mL}$ of blood loss in the first hour in the intensive care unit, and more than $100 \mathrm{~mL} /$ hour during further ICU stay. The final decision whether or not a ROTEM test should be performed was made by the anaesthesiologist or intensivist in charge. ROTEM-based transfusion algorithms were adopted from literature. ${ }^{10}$ Data acquisition and analyses were performed anonymously in accordance with the Dutch law for approving medical research.

Blood was collected in $4.0 \mathrm{~mL}$ tubes with $7.2 \mathrm{mg}$ spray dried K2-EDTA for analysis of platelet count, and in $4.5 \mathrm{~mL}, 3.2 \%$ citrate tubes for ROTEM tests and analysis of fibrinogen (BD Vacutainer, Becton Dickinson, Franklin Lakes, NJ). Platelet measurements were performed on a Sysmex XE-5000 analyzer (Sysmex Corporation, Kobe, Japan), and fibrinogen measurements were performed on a Sysmex CA-7000 analyzer after centrifugation for 10 minutes at 2,000 g. Fibrinogen levels were determined using the Clauss coagulation method ${ }^{15}$ (Dade Thrombin Reagent, Siemens Healthcare Diagnostics Products, Marburg, Germany). 
Thromboelastometric measurements were performed with the ROTEM device (TEM International $\mathrm{GmbH}$, Munich, Germany). Measurements were performed at $37^{\circ} \mathrm{C}$ according to the instructions of the manufacturer. Analysed ROTEM parameters were EXTEM A5/A10/MCF, FIBTEM A5/A10/MCF, and PLTEM A5/A10/MCF, the last of which were calculated by the investigator by subtracting FIBTEM from EXTEM. In EXTEM and FIBTEM tests, coagulation is extrinsically activated by tissue factor. In the FIBTEM tests a platelet inhibitor is added (cytochalasin D) to correct for the contribution of platelets to the clot strength. ROTEM tests and conventional laboratory tests were performed simultaneously in the central laboratory by trained laboratory staff. Both conventional laboratory tests and ROTEM tests were part of the standard diagnostic procedure during cardiac surgery and were therefore, performed, instantly.

From October 2010 until October 2012, the turnaround time of conventional laboratory tests was retrospectively analysed in the central laboratory for emergency requests (<30 minutes). Turn-around time was defined as the time that passed between arrival of the sample at the laboratory, after pneumatic tube transport, and the moment results were reported in the laboratory information system. Handling time of 1 single ROTEM request was analysed perspectively. Time to perform 1 ROTEM test was calculated by collecting data of 264 ROTEM tests. Pneumatic tube transport time from the operating room to the laboratory was not included in this study but has been reported to be equal for all sample transports (between 83 and 100 seconds). ${ }^{16}$

Continuous data are shown as mean and standard deviation (SD) when data followed normal distribution and median plus interquartile range (IQR) when skewed distribution was apparent. Categorical data are shown in absolute numbers and percentages unless otherwise stated. Pearson's correlation coefficient ( $r$ ) was calculated to determine the correlation between ROTEM variables and conventional laboratory tests and for comparison of A5, A10, and MCF values in EXTEM, FIBTEM, and PLTEM. When multiple ROTEM tests were performed in the same patient, the first ROTEM test was used for statistical analysis. A Fisher r-to-z transformation was performed to statistically compare correlation coefficients. Areas under the curves of receiver operating characteristic (ROC) 


\section{Chapter 6}

curves were calculated to compare the ability of FIBTEM A5, A10, and MCF to predict hypofibrinogenemia and for the ability of EXTEM A5, A10, and MCF and PLTEM A5, A10, and MCF to predict thrombocytopenia. Areas under the curves were compared with each other by using a method described by Hanley et al, which accounts for the fact that areas under the curves are derived from the same sample of patients. ${ }^{17}$ To evaluate the clinical significance of ROTEM tests and determine the value of ROTEM thresholds for detecting thrombocytopenia and hypofibrinogenemia, sensitivity, specificity and positive and negative predictive value (PPV, NPV) were calculated. All statistical analyses were performed using computer software (SPSS, Version 20.0, SPSS, Inc., Chicago, IL).

\section{Results}

ROTEM results were obtained from 97 patients who underwent cardiac surgery. A total of 140 ROTEM results were collected from these patients of which 38 results were obtained during $\mathrm{CPB}$ and 102 results were obtained after CPB.

Table 6.1 Patient characteristics, laboratory results, and ROTEM results ${ }^{\pi}$

\begin{tabular}{|c|c|c|}
\hline Patient characteristics & $\begin{array}{c}\text { Sex (male/female) } \\
\text { Age (years) }\end{array}$ & $\begin{array}{c}70 / 27 \\
62(14)\end{array}$ \\
\hline Laboratory results & $\begin{array}{c}\text { Haemoglobin }(\mathrm{mmol} / \mathrm{L}) \\
\text { Haematocrit }(\mathrm{L} / \mathrm{L}) \\
\text { Platelet count }\left(\times 10^{9} / \mathrm{L}\right) \\
\text { Fibrinogen }(\mathrm{g} / \mathrm{L})\end{array}$ & $\begin{array}{c}5.4(0.9) \\
0.26(0.04) \\
123(50) \\
2.0(0.9)\end{array}$ \\
\hline ROTEM results & $\begin{array}{l}\text { EXTEM A5 }(\mathrm{mm}) \\
\text { EXTEM A10 }(\mathrm{mm}) \\
\text { EXTEM MCF }(\mathrm{mm}) \\
\text { FIBTEM A5 }(\mathrm{mm}) \\
\text { FIBTEM A10 }(\mathrm{mm}) \\
\text { FIBTEM MCF }(\mathrm{mm}) \\
\text { PLTEM A5 }(\mathrm{mm}) \\
\text { PLTEM A10 }(\mathrm{mm}) \\
\text { PLTEM MCF }(\mathrm{mm})\end{array}$ & $\begin{array}{l}34(9) \\
45(9) \\
55(8) \\
9(5) \\
10(6) \\
11(6) \\
25(5) \\
35(6) \\
43(5)\end{array}$ \\
\hline $\begin{array}{l}\text { Total number of transfusions during the } 24 \text { hours } \\
\text { before/after the ROTEM test (EC/FFP/PC) }\end{array}$ & $\begin{array}{c}\text { Before } \\
\text { After } \\
\end{array}$ & $\begin{array}{l}601(399 / 134 / 68) \\
349(144 / 140 / 65) \\
\end{array}$ \\
\hline $\begin{array}{l}\text { Patients who received at least one transfusion } \\
\text { before performing a ROTEM test }\end{array}$ & $\begin{array}{l}\text { EC } \\
\text { FFP } \\
\text { PC }\end{array}$ & $\begin{array}{l}78 / 97 \\
26 / 97 \\
39 / 97\end{array}$ \\
\hline
\end{tabular}

Data are expressed as mean (SD) or number as appropriate.

Abbreviations: $\mathrm{A}(\mathrm{x})$, maximal clot formation after $(\mathrm{x})$ minutes; $\mathrm{CPB}$, cardiopulmonary bypass; $\mathrm{EC}$, erythrocyte concentrate; EXTEM, extrinsically-activated ROTEM test; FIBTEM, fibrin-specific ROTEM test; FFP, fresh frozen plasma; MCF, maximal clot formation; PC, platelet concentrate; $\operatorname{PLTEM}(x), \operatorname{EXTEM}(x)$ - FIBTEM(x); ROTEM, rotational thromboelastometry; SD, standard deviation 
Mean laboratory values revealed low haemoglobin, haematocrit, and platelet count (table 6.1). Mean ROTEM results were within normal range. In the 48hour period around the first ROTEM test, 97 patients received 950 blood products: 543 red blood cell (RBC) concentrates, 274 fresh frozen plasma (FFP), and 133 platelet concentrates. Eighty-one (84\%) patients already were transfused prior to performing a ROTEM test, when 39 (40\%) patients received at least 1 platelet concentrate and $26(27 \%)$ patients received at least 1 FFP (table 6.1 ). Thirty-seven patients $(38 \%)$ received $\geq 10$ transfusions, and 9 patients received $\geq 25$ transfusions during this period.

Table 6.2 Overview of Pearson's correlation coefficients describing the correlation among ROTEM tests and between ROTEM tests and conventional laboratory tests

\begin{tabular}{|c|c|c|c|c|c|c|c|c|}
\hline & \multicolumn{6}{|c|}{ ROTEM tests } & \multicolumn{2}{|c|}{ Conventional tests } \\
\hline & \multicolumn{2}{|c|}{ EXTEM } & \multicolumn{2}{|c|}{ FIBTEM } & \multicolumn{2}{|c|}{ PLTEM } & \multirow{2}{*}{ PLT } & \multirow{2}{*}{ Fibrinogen } \\
\hline & A10 & MCF & A10 & MCF & A10 & MCF & & \\
\hline \multicolumn{9}{|l|}{ EXTEM } \\
\hline A5 & 0.99 & 0.97 & - & - & - & - & 0.74 & - \\
\hline A10 & - & 0.98 & - & - & - & - & 0.73 & - \\
\hline MCF & 0.98 & - & - & - & - & - & 0.70 & - \\
\hline \multicolumn{9}{|l|}{ FIBTEM } \\
\hline A5 & - & - & 1.00 & 0.99 & - & - & - & 0.87 \\
\hline A10 & - & - & - & 0.99 & - & - & - & 0.86 \\
\hline MCF & - & - & 0.99 & - & - & - & - & 0.87 \\
\hline \multicolumn{9}{|l|}{ PLTEM } \\
\hline A5 & - & - & - & - & 0.98 & 0.88 & 0.85 & - \\
\hline A10 & - & - & - & - & - & 0.92 & 0.82 & - \\
\hline MCF & - & - & - & - & 0.92 & - & 0.71 & - \\
\hline
\end{tabular}

Abbreviations: $\mathrm{A}(\mathrm{x})$, amplitude at $(\mathrm{x})$ minutes; EXTEM, extrinsically-activated thromboelastometry; FIBTEM, fibrin-specific thromboelastometry; MCF, maximal clot formation; PLTEM(x), EXTEM(x) FIBTEM(x); PLT, platelet count; ROTEM, rotational thromboelastometry; (-), data not calculated.

Both EXTEM A5 and FIBTEM A5 had an excellent linear correlation with A10 ( $r$ $=0.99 / 1.00 ; p<0.001)$ and MCF $(r=0.97 / 0.99 ; p<0.001)$. EXTEM A5 and FIBTEM A5 also were well correlated with platelet count $(r=0.74 ; p<0.001)$ and fibrinogen level $(r=0.87 ; p<0.001)$, respectively. Correlation of EXTEM A5 and FIBTEM A5 was comparable with A10 (EXTEM, $p=0.90$; FIBTEM, $p=$ 1.00 ) and MCF (EXTEM, $p=0.64$; FIBTEM, $p=0.83$ ) (figure 6.1). The calculated parameter PLTEM A5 $(r=0.85)$ showed a better correlation with platelet count than EXTEM A5 ( $r=0.74 ; p=0.04)$, in which the contribution of fibrinogen is not eliminated (table 6.2). Correlation among all of these parameters did 
A

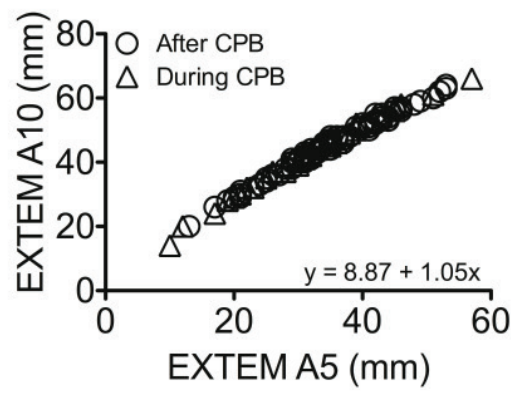

C

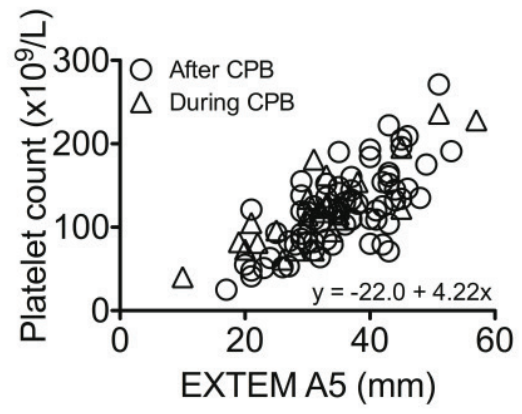

E

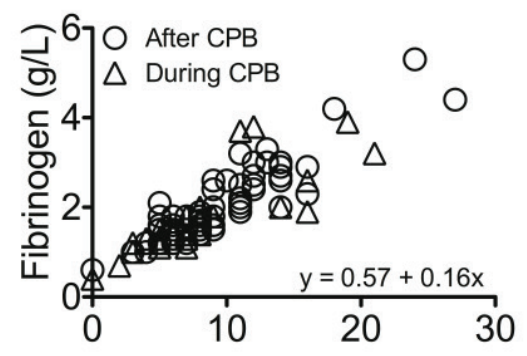

FIBTEM A5 (mm)
B
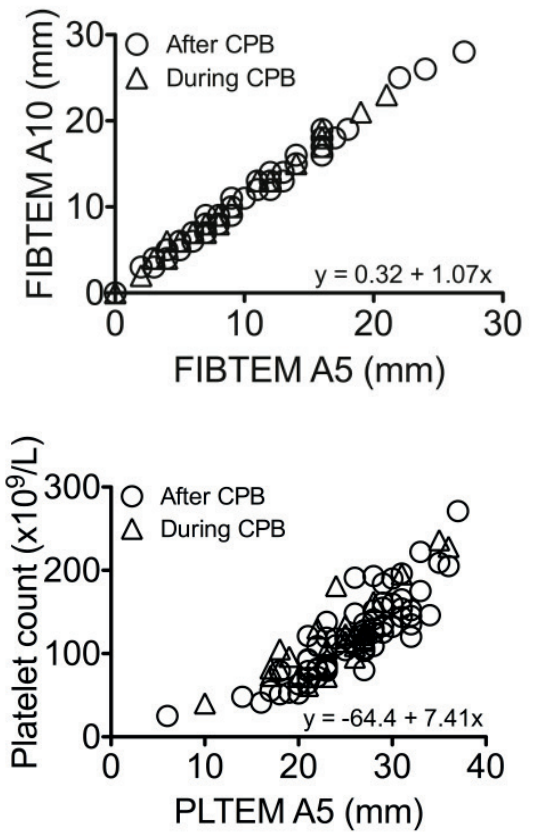

Figure 6.1 Correlation among ROTEM parameters (A, B) and between ROTEM parameters and conventional laboratory tests (C, D and $\mathrm{E})$.

$\triangle$, during $\mathrm{CPB}$; $\mathrm{O}$, after $\mathrm{CPB} ; \mathrm{A}(\mathrm{x})$, amplitude at $(\mathrm{x})$ minutes; $\mathrm{CPB}$, cardiopulmonary bypass; EXTEM, extrinsically-activated thromboelasticity; FIBTEM, fibrin-specific thromboelasticity; PLTEM(x), EXTEM(x) $\operatorname{FIBTEM}(x)$.

not differ in the subgroup of massively transfused patients or in tests performed during CPB.

Areas under the curves of ROC analyses were calculated to evaluate the predictive value of ROTEM tests for thrombocytopenia and hypofibrinogenemia (table 6.3). Area under the curve of PLTEM A5/A10 was superior to EXTEM A5/A10 for the threshold of $80 \times 10^{9} / L(A 5 p=0.025, A 10 p=0.019)$ and 100 $\mathrm{x} 10^{9} / \mathrm{L}$ (A5 $p=0.012, \mathrm{~A} 10 p=0.019$ ) (figure 6.2). For FIBTEM A5/A10/MCF, area under the curve was calculated for fibrinogen levels of $<1.2 \mathrm{~g} / \mathrm{L},<1.5 \mathrm{~g} / \mathrm{L}$, 
and $<2.0 \mathrm{~g} / \mathrm{L}$ and appeared to be comparable for A5 $(0.93,0.90,0.95)$, A10 $(0.93,0.89,0.95)$, and $\operatorname{MCF}(0.93,0.89,0.96)$.

Median turnaround times (IQR) of emergency laboratory requests were 13 minutes (9-22) for platelet count and 37 minutes (31-54) for fibrinogen levels. Median ROTEM handling time in the central laboratory was 4.5 minutes (4-7) and median time to perform 1 ROTEM test was 76 seconds (57-84). When the ROTEM test has started, it takes another 6 minutes, (clotting time around 1 minute and another 5 minutes until A5), before A5 results are known. Total turnaround time of EXTEM A5 or FIBTEM A5 was therefore approximately 12 minutes.

Table 6.3 Areas under the curves $(95 \% \mathrm{Cl})$ of receiver-operating characteristic curves for different cut-off values of platelet count and fibrinogen level for EXTEM (A), PLTEM (A) and FIBTEM (B)

\begin{tabular}{|c|c|c|}
\hline \multirow{2}{*}{ A } & \multicolumn{2}{|c|}{ Platelet count $\left(\times 10^{9} / \mathrm{L}\right)$} \\
\hline & $<80$ & $<100$ \\
\hline \multicolumn{3}{|l|}{ EXTEM } \\
\hline A5 & $0.83(0.71-0.94)$ & $0.87(0.79-0.95)$ \\
\hline A10 & $0.82(0.70-0.90)$ & $0.85(0.77-0.94)$ \\
\hline MCF & $0.82(0.71-0.94)$ & $0.85(0.77-0.94)$ \\
\hline \multicolumn{3}{|l|}{ PLTEM } \\
\hline A5 & $0.95(0.90-0.99)^{*}$ & $0.95(0.91-0.99)^{\dagger}$ \\
\hline A10 & $0.94(0.90-0.99)^{\ddagger}$ & $0.93(0.88-0.99)^{\S}$ \\
\hline MCF & $0.94(0.90-0.99)$ & $0.92(0.86-0.98)$ \\
\hline \multirow{2}{*}{ B } & \multicolumn{2}{|c|}{ Fibrinogen level (g/L) } \\
\hline & $<1.2$ & $<2.0$ \\
\hline \multicolumn{3}{|l|}{ FIBTEM } \\
\hline A5 & $0.93(0.81-1.00)$ & $0.95(0.90-1.00)$ \\
\hline A10 & $0.93(0.86-1.00)$ & $0.95(0.90-1.00)$ \\
\hline MCF & $0.93(0.85-1.00)$ & $0.96(0.91-1.00)$ \\
\hline
\end{tabular}

${ }^{*} p=0.025$ for comparable EXTEM values

${ }^{\dagger} p=0.019$ for comparable EXTEM values

$\ddagger p=0.012$ for comparable EXTEM values

$\S p=0.019$ for comparable EXTEM values

Abbreviations: $\mathrm{A}(\mathrm{x})$, amplitude at $(\mathrm{x})$ minutes; EXTEM, extrinsically-activated thromboelastometry; FIBTEM, fibrin-specific thromboelastometry; MCF, maximal clot formation; PLTEM(x), EXTEM(x) $\operatorname{FIBTEM}(\mathrm{x})$.

In this study, a previously reported EXTEM threshold for determining acute coagulopathy $(35 \mathrm{~mm})$ missed $5 \%$ of patients with platelet counts $<80 \times 10^{9} / \mathrm{L} .{ }^{4}$ However, only $30 \%$ (PPV) of patients with EXTEM A5 $<35 \mathrm{~mm}$ actually had a platelet count $<80 \times 10^{9} / \mathrm{L}$. A calculated threshold of PLTEM $(22 \mathrm{~mm})$ missed a comparable number of patients with platelet counts $<80 \times 10^{9} / \mathrm{L}(6 \%)$ but showed a much better PPV of $70 \%$ (table 6.4). A $9.6 \mathrm{~mm}$ FIBTEM threshold, which was 
A

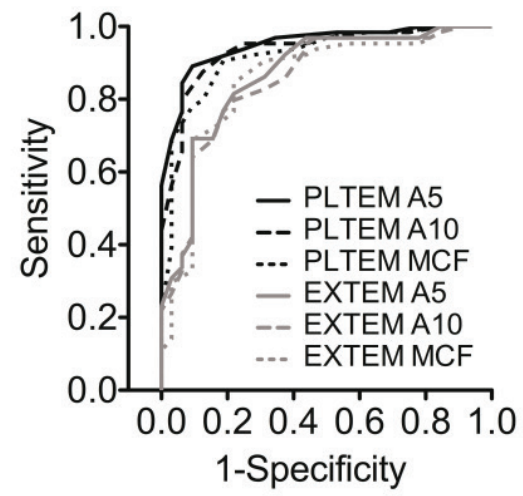

B

Fibrinogen level $<1.5 \mathrm{~g} / \mathrm{L}$

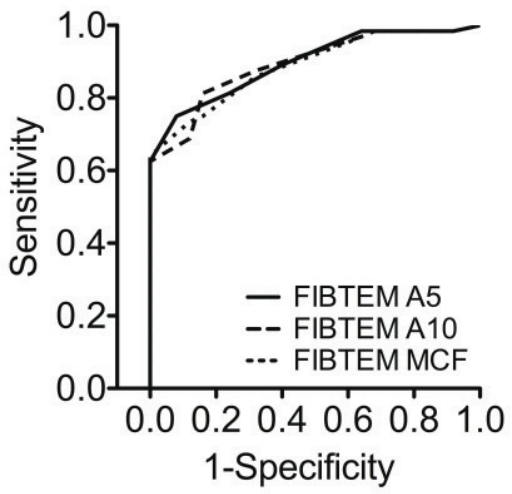

Figure 6.2 Receiver-operating characteristic curves of the ability of EXTEM and PLTEM to predict thrombocytopenia $\left(<100 \times 10^{9} / \mathrm{L}\right)$ and FIBTEM to predict hypofibrinogenemia $(<1.5 \mathrm{~g} / \mathrm{L})$. PLTEM A5 and $\mathrm{A} 10$ are superior in predicting thrombocytopenia compared with EXTEM A5 $(p=0.012)$ and A10 $(p=0.019)$. A $(x)$, amplitude at $(x)$ minutes; EXTEM, extrinsically-activated thromboelasticity; FIBTEM, fibrin-specific thromboelasticity; MCF, maximal clot formation; $\operatorname{PLTEM}(x), \operatorname{EXTEM}(x)$ $\operatorname{FIBTEM}(\mathrm{x})$.

Table 6.4 Sensitivity, specificity, PPV and NPV (\%) of ROTEM parameters for hypofibrinogenemia and thrombocytopenia

\begin{tabular}{|c|c|c|c|c|c|c|}
\hline & \multicolumn{3}{|c|}{ Fibrinogen $(\mathrm{g} / \mathrm{L})$} & \multicolumn{3}{|c|}{ Platelet count $\left(\times 10^{9} / \mathrm{L}\right)$} \\
\hline & $\begin{array}{l}<1.2 \\
(\mathrm{n}=8)\end{array}$ & $\begin{array}{c}<1.5 \\
(n=26)\end{array}$ & $\begin{array}{c}<2.0 \\
(n=85)\end{array}$ & $\begin{array}{c}<80 \\
(n=19)\end{array}$ & $\begin{array}{c}<100 \\
(n=32)\end{array}$ & $\begin{array}{c}<150 \\
(n=74)\end{array}$ \\
\hline \multicolumn{7}{|l|}{$\begin{array}{l}\text { FIBTEM A5 } \\
<9.5 \mathrm{~mm}^{27}\end{array}$} \\
\hline Sensitivity & 100 & 100 & 94 & - & - & - \\
\hline Specificity & 42 & 53 & 86 & - & - & - \\
\hline PPV & 15 & 46 & 91 & - & - & - \\
\hline NPV & 100 & 100 & 91 & - & - & - \\
\hline \multicolumn{7}{|l|}{$\begin{array}{l}\text { EXTEM A5 } \\
<36 \mathrm{~mm}^{26}\end{array}$} \\
\hline Sensitivity & 100 & 92 & 84 & 89 & 91 & 69 \\
\hline Specificity & 47 & 57 & 83 & 50 & 58 & 78 \\
\hline PPV & 15 & 46 & 88 & 30 & 52 & 91 \\
\hline NPV & 100 & 95 & 77 & 95 & 93 & 44 \\
\hline \multicolumn{7}{|l|}{ PLTEM A5 } \\
\hline Sensitivity & - & _ & ـ & 84 & 66 & 31 \\
\hline Specificity & - & - & - & 91 & 97 & 100 \\
\hline PPV & - & - & - & 70 & 91 & 100 \\
\hline NPV & - & - & - & 96 & 85 & 30 \\
\hline
\end{tabular}


shown to identify acute traumatic haemorrhage in a previous study, identified all patients with fibrinogen levels $>1.5 \mathrm{~g} / \mathrm{L} .{ }^{26}$ However, only $54 \%$ of patients with FIBTEM A5 $>9.5 \mathrm{~mm}$ had fibrinogen levels $<1.5 \mathrm{~g} / \mathrm{L}$, and $9 \%$ of patients even had fibrinogen levels $>2.0 \mathrm{~g} / \mathrm{L}$ (table 6.4).

\section{Discussion}

In the present study, EXTEM A5 and FIBTEM A5 showed an excellent correlation with $\mathrm{A} 10$ and MCF. Consequently, EXTEM A5, A10, and MCF showed a comparable correlation with platelet count, and FIBTEM A5, A10, and MCF were similarly correlated with fibrinogen levels. In addition, A5, A10, and MCF had the same ability to predict clinically relevant thrombocytopenia and hypofibrinogenemia during cardiac surgery.

ROTEM increasingly is used to assess haemostasis both during situations of acute blood loss as well as situations of controlled blood loss such as elective surgery. A major advantage of ROTEM is that tests are performed in whole blood which allows for point-of-care testing immediately after blood collection. In contrast to conventional laboratory tests, the contribution of blood cells to clot formation also is taken into account in ROTEM tests. In addition, ROTEM provides valuable information about fibrinolysis. ROTEM-guided transfusion protocols have been reported to reduce the amount of blood loss and the incidence of blood transfusion when compared with protocols guided by conventional laboratory tests. ${ }^{9-11}$ At this moment, ROTEM-guided transfusion protocols use EXTEM A10 and FIBTEM A10 to guide transfusion of platelet and fibrinogen concentrates. ${ }^{6,10}$ This study, however, showed that the turnaround time could be reduced safely by 5 minutes by replacing A10 with A5 without losing any accuracy in detecting thrombocytopenia and hypofibrinogenemia. The results of this study were in line with data from other studies that described that A5 and A10 were reliable predictors of MCF in non-cardiac surgery. ${ }^{18}$ Another recent study showed that these early values of clot firmness reliably predicted MCF in patients undergoing CPB surgery both during CPB and after CPB. ${ }^{19}$ The authors' results independently supported the use of early ROTEM parameters to guide transfusion management. Since ROTEM has been reported to be a reliable test 


\section{Chapter 6}

when operated by trained non-laboratory staff, the turnaround time in this study could have been reduced further by another 83-100 seconds if tests had been performed point-of-care. ${ }^{16,20}$

Another important observation of this study concerned a calculated parameter, PLTEM. The EXTEM test, which has been implemented in ROTEM-guided transfusion protocols to guide transfusion of platelet concentrates, is influenced by at least 2 parameters, namely platelet count and fibrinogen level. ${ }^{6,10}$ By subtracting FIBTEM from EXTEM, the authors eliminated the influence of fibrinogen on EXTEM. This platelet component in the EXTEM test had been mentioned before but had not yet been related to conventional laboratory tests. ${ }^{21-23}$ PLTEM appeared to be more accurate in estimating platelet counts and thrombocytopenia than EXTEM. Implementation of PLTEM in ROTEM-guided transfusion protocols may, therefore, be an important new parameter in cardiac surgery.

Because platelets in EXTEM tests are activated via the plasmatic generation of thrombin, which is a very potent platelet activator, platelet dysfunction during cardiac surgery cannot be detected with ROTEM tests. Therefore, platelet function tests, using a panel of weak and strong agonists, still need to be performed next to ROTEM tests to obtain information about platelet function.

During this study in 97 cardiac surgical patients, only 4 patients with a platelet count $<50 \times 10^{9} / \mathrm{L}$ and 6 patients with a fibrinogen level $\leq 1.0 \mathrm{~g} / \mathrm{L}$ were included. Although laboratory results suggested that a severe coagulopathy was only apparent in these patients, the incidence may be higher since $38 \%$ received $\geq 10$ transfusions, and $9 \%$ were transfused with $\geq 25$ products. Moreover, these small numbers of patients with very low platelet counts and fibrinogen levels could be explained by the fact that $84 \%$ of patients received at least 1 transfusion before the first ROTEM test was performed, indicating the need for a more rapid test that may reduce the number of empirical transfusions. Correlations between ROTEM parameters and conventional laboratory tests were not affected by massive transfusion.

Transfusion thresholds of fibrinogen in cardiac surgery vary widely and depend on clinical parameters such as the presence of bleeding. ${ }^{24}$ The American Guideline of Perioperative Blood Transfusion suggests a fibrinogen concentra- 
tion of $<0.8$ to $1.0 \mathrm{~g} / \mathrm{L}$ before transfusion of cryoprecipitate; while the European Guideline of Bleeding Following Major Trauma raised the threshold for transfusion of fibrinogen from $<1.0 \mathrm{~g} / \mathrm{L}$ in 2007 to $<1.5$ to $2.0 \mathrm{~g} / \mathrm{L}$ in 2010 . $^{12,13}$ This is more in line with an in vitro model of haemodilution that showed that an optimal rate of clot formation was not achieved until fibrinogen levels $>2.0 \mathrm{~g} / \mathrm{L}$. ${ }^{25}$ Both guidelines agree about an absolute transfusion trigger of platelet concentrates when platelet counts fell below $50 \times 10^{9} / \mathrm{L}^{12,13}$ The decision to transfuse a patient with a platelet count between 50 and $100 \times 10^{9} / \mathrm{L}$ should be based on the potential for platelet dysfunction and on anticipated or on-going bleeding. ${ }^{12}$ Since there is no consensus about transfusion thresholds and since these thresholds also depend on clinical parameters, definite transfusion thresholds for ROTEM A5 parameters remain to be defined. Before ROTEM thresholds can be implemented in transfusion management strategies, they, therefore, have to be adjusted to the desired transfusion thresholds and the population of interest. Also, data on sensitivity and specificity of ROTEM thresholds have to be adjusted accordingly.

There were several limitations of this study. Since the indications for ROTEM tests in this study have selected high-risk patients, the authors' assumptions need to be validated in the general population of patients undergoing cardiac surgery. On the other hand, high-risk patients will be the most important subgroup because of the expected higher incidence of thrombocytopenia and hypofibrinogenemia. Secondly, the number of patients with a platelet count $<50$ $x 10^{9} / \mathrm{L}$, the absolute transfusion trigger in cardiac surgery, was too low to draw any conclusions about the ability of EXTEM A5 and PLTEM A5 to estimate platelet counts $<50 \times 10^{9} / \mathrm{L}$. This, however, represents daily clinical practice, in which patients already have received multiple transfusions before conventional laboratory results are reported. Thirdly, ROTEM results are not influenced by endothelial responses and the impact of blood flow, which also were considered important factors in haemostasis in vivo.

ROTEM-guided transfusion protocols have shown previously to decrease postoperative blood loss and transfusion requirements in cardiac surgery. ${ }^{9,10}$ Since transfusions during cardiac surgery are associated with increased short-term 


\section{Chapter 6}

and long-term mortality, unwarranted use of blood products should be avoided. ${ }^{27}$ Introduction of A5 values in these transfusion protocols allows clinicians to decide 5 minutes earlier whether transfusion of platelet concentrates or fibrinogen is necessary, without losing any sensitivity or specificity. ROTEM-guided transfusion protocols can be optimized further when EXTEM is replaced by PLTEM because of its more accurate estimation of platelet count and thrombocytopenia. Implementation of both PLTEM A5 and FIBTEM A5, therefore, might prevent suboptimal transfusions in cardiac surgery. Further studies are needed to evaluate whether implementation of FIBTEM A5 and PLTEM A5 in ROTEMguided transfusion protocols will result in improved transfusion management in cardiac surgery. 


\section{References}

1. Johansson PI, Sølbeck S, Genet G, Stensballe J, Ostrowski SR. Coagulopathy and hemostatic monitoring in cardiac surgery: an update. Scand Cardiovasc J. 2012 Aug;46(4):194-202

2. Bolliger D, Szlam F, Levy JH, Molinaro RJ, Tanaka KA. Haemodilution-induced profibrinolytic state is mitigated by fresh-frozen plasma: implications for early haemostatic intervention in massive haemorrhage. Br J Anaesth. 2010 Mar;104(3):318-25.

3. Tanaka KA, Bolliger D, Vadlamudi R, Nimmo A. Rotational thromboelastometry (ROTEM)based coagulation management in cardiac surgery and major trauma. J Cardiothorac Vasc Anesth. 2012 Dec;26(6):1083-93.

4. Davenport R, Manson J, De'Ath H, Platton S, Coates A, Allard S, Hart D, Pearse R, Pasi KJ, MacCallum P, Stanworth S, Brohi K. Functional definition and characterization of acute traumatic coagulopathy. Crit Care Med. 2011 Dec;39(12):2652-8.

5. Ogawa S, Szlam F, Chen EP, Nishimura T, Kim H, Roback JD, Levy JH, Tanaka KA. A comparative evaluation of rotation thromboelastometry and standard coagulation tests in hemodilution-induced coagulation changes after cardiac surgery. Transfusion. 2012 Jan;52(1):14-22.

6. Girdauskas E, Kempfert J, Kuntze T, Borger MA, Enders J, Fassl J, Falk V, Mohr FW. Thromboelastometrically guided transfusion protocol during aortic surgery with circulatory arrest: a prospective, randomized trial. J Thorac Cardiovasc Surg. 2010 Nov;140(5):1117-24.e2.

7. Roullet S, Pillot J, Freyburger G, Biais M, Quinart A, Rault A, Revel P, Sztark F. Rotation thromboelastometry detects thrombocytopenia and hypofibrinogenaemia during orthotopic liver transplantation. Br J Anaesth. 2010 Apr;104(4):422-8.

8. Blasi A, Beltran J, Pereira A, Martinez-Palli G, Torrents A, Balust J, Zavala E, Taura P, GarciaValdecasas JC. An assessment of thromboelastometry to monitor blood coagulation and guide transfusion support in liver transplantation. Transfusion. 2012 Sep;52(9):1989-98.

9. Afshari A, Wikkelsø A, Brok J, Møller AM, Wetterslev J. Thrombelastography (TEG) or thromboelastometry (ROTEM) to monitor haemotherapy versus usual care in patients with massive transfusion. Cochrane Database Syst Rev. 2011 Mar 16;(3):CD007871.

10. Görlinger K, Dirkmann D, Hanke AA, Kamler M, Kottenberg E, Thielmann M, Jakob H, Peters J. First-line therapy with coagulation factor concentrates combined with point-of-care coagulation testing is associated with decreased allogeneic blood transfusion in cardiovascular surgery: a retrospective, single-center cohort study. Anesthesiology. 2011 Dec;115(6):1179-91.

11. Weber CF, Görlinger K, Meininger D, Herrmann E, Bingold T, Moritz A, Cohn LH, Zacharowski K. Point-of-care testing: a prospective, randomized clinical trial of efficacy in coagulopathic cardiac surgery patients. Anesthesiology. 2012 Sep;117(3):531-47.

12. American Society of Anesthesiologists Task Force on Perioperative Blood Transfusion and Adjuvant Therapies. Practice guidelines for perioperative blood transfusion and adjuvant therapies: an updated report by the American Society of Anesthesiologists Task Force on Perioperative Blood Transfusion and Adjuvant Therapies. Anesthesiology. 2006 Jul;105(1):198-208. 


\section{Chapter 6}

13. Rossaint R, Bouillon B, Cerny V, Coats TJ, Duranteau J, Fernández-Mondéjar E, Hunt BJ, Komadina R, Nardi G, Neugebauer E, Ozier Y, Riddez L, Schultz A, Stahel PF, Vincent JL, Spahn DR. Management of bleeding following major trauma: an updated European guideline. Crit Care. 2010;14(2):R52.

14. Chandler WL, Ferrell C, Trimble S, Moody S. Development of a rapid emergency hemorrhage panel. Transfusion. 2010 Dec;50(12):2547-52.

15. Clauss A. [Rapid physiological coagulation method in determination of fibrinogen]. Acta Haematol. 1957 Apr;17(4):237-46.

16. Lancé MD, Kuiper GJ, Sloep M, Spronk HM, van Oerle R, ten Cate H, Marcus MA, Henskens YM. The effects of pneumatic tube system transport on ROTEM analysis and contact activation assessed by thrombin generation test. Thromb Res. 2012 Sep;130(3):e147-50.

17. Hanley JA, McNeil BJ. A method of comparing the areas under receiver operating characteristic curves derived from the same cases. Radiology. 1983 Sep;148(3):839-43.

18. Görlinger K, Dirkmann D, Solomon C, Hanke AA. Fast interpretation of thromboelastometry in non-cardiac surgery: reliability in patients with hypo-, normo-, and hypercoagulability. $\mathrm{Br} \mathrm{J}$ Anaesth. 2013 Feb;110(2):222-30.

19. Dirkmann D, Görlinger K, Dusse F, Kottenberg E, Peters J. Early thromboelastometric variables reliably predict maximum clot firmness in patients undergoing cardiac surgery: a step towards earlier decision making. Acta Anaesthesiol Scand. 2013 May;57(5):594-603.

20. Mauch J, Spielmann N, Hartnack S, Madjdpour C, Kutter AP, Bettschart-Wolfensberger R, Weiss M, Haas T. Intrarater and interrater variability of point of care coagulation testing using the ROTEM delta. Blood Coagul Fibrinolysis. 2011 Dec;22(8):662-6.

21. Lang T, Johanning K, Metzler H, Piepenbrock S, Solomon C, Rahe-Meyer N, Tanaka KA. The effects of fibrinogen levels on thromboelastometric variables in the presence of thrombocytopenia. Anesth Analg. 2009 Mar; 108(3):751-8.

22. Kettner SC, Panzer OP, Kozek SA, Seibt FA, Stoiser B, Kofler J, Locker GJ, Zimpfer M. Use of abciximab-modified thrombelastography in patients undergoing cardiac surgery. Anesth Analg. 1999 Sep;89(3):580-4.

23. Kuitunen AH, Suojaranta-Ylinen RT, Kukkonen SI, Niemi TT. Tranexamic acid does not correct the haemostatic impairment caused by hydroxyethyl starch (200 kDa/0.5) after cardiac surgery. Blood Coagul Fibrinolysis. 2006 Nov; 17(8):639-45

24. Likosky DS, FitzGerald DC, Groom RC, Jones DK, Baker RA, Shann KG, Mazer CD, Spiess BD, Body SC. Effect of the perioperative blood transfusion and blood conservation in cardiac surgery clinical practice guidelines of the Society of Thoracic Surgeons and the Society of Cardiovascular Anesthesiologists upon clinical practices. Anesth Analg. 2010 Aug;111(2):316-23.

25. Bolliger D, Szlam F, Molinaro RJ, Rahe-Meyer N, Levy JH, Tanaka KA. Finding the optimal concentration range for fibrinogen replacement after severe haemodilution: an in vitro model. $\mathrm{Br}$ J Anaesth. 2009 Jun;102(6):793-9. 
26. Rourke C, Curry N, Khan S, Taylor R, Raza I, Davenport R, Stanworth S, Brohi K. Fibrinogen levels during trauma hemorrhage, response to replacement therapy, and association with patient outcomes. J Thromb Haemost. 2012 Jul;10(7):1342-51.

27. Bhaskar B, Dulhunty J, Mullany DV, Fraser JF. Impact of blood product transfusion on short and long-term survival after cardiac surgery: more evidence. Ann Thorac Surg. 2012 Aug;94(2):4607. 



\section{Chapter 7}

\section{Shifts of transfusion demand in}

cardiac surgery after implementation

of rotational thromboelastometry

guided transfusion protocols: analysis of the HEROES-CS

(haemostasis registry of patients in cardiac surgery) observational prospective open cohort database

Kuiper GJ, van Egmond LT, Henskens YM, Roekaerts PM, Maessen JG, Ten Cate H, Buhre WF, Lance MD

Submitted. 


\section{Abstract}

\section{Background}

ROTEM-guided transfusion algorithms in cardiac surgery have proven to be successful in reducing blood loss in randomized controlled trials. Using HEROES-CS (haemostasis registry of patients in cardiac surgery) as the first prospective cohort study on this matter, we hope to show that ROTEMguided transfusion algorithms, save blood products, save lives, and overall costs in cardiac surgery in every day practice.

\section{Methods}

Two cohorts of cardiac surgery patients were prospectively followed in our academic hospital. In the first cohort a classical-guided algorithm and in the second a ROTEM-guided algorithm was used for patient blood management. Primary outcome was the use and amount of blood products and haemostatic medication. Secondary outcomes were amount of rethoracotomies, length of stay, and 30day mortality. Finally, costs and savings were calculated.

\section{Results}

Two hundred and four patients were included in the classical-guided and 151 in the ROTEM-guided cohort. Baseline characteristics showed excellent similarities. Blood loss was lower after ROTEMguidance $(p<0.005)$. Absolute risk reduction was $11 \%$ for red blood cell use $(p=0.041), 11 \%$ for fresh frozen plasma $(p=0.003)$, and $7 \%$ for thrombocyte concentrate $(p=0.121)$. More tranexamic acid and fibrinogen concentrate were given in expense, while desmopressin was given less often. Hospital length of stay was reduced by an overall median of 2 and a mean of 3 days $(p<0.001)$. Mortality and rethoracotomy rates were not affected. Potential savings were estimated to be around 3.4 thousand Euro per patient.

\section{Conclusions}

Implementation of a ROTEM-guided transfusion algorithm in cardiac surgery patients reduced use of blood products and haemostatic medication, hereby saving costs. Reductions in mortality and rethoracotomy rates could not be found. 


\section{Chapter 7}

\section{Introduction}

Transfusion of blood components is basically a form of tissue allotransplantation. The safety of transfusion is of utmost concern, because, despite strict protocols and surveillance in production and transfusion, adverse reactions still occur. ${ }^{1}$ Transfusion-associated circulatory overload (TACO) and transfusion related fever are more common than death by a medical error. ${ }^{2}$ The best-known indications for transfusion of packed red blood cells, fresh frozen, plasma, and thrombocyte concentrates is in significant blood loss due to trauma and/or major surgery.

Traditionally, the clinical decision for transfusion of blood products is guided by algorithms and guidelines based on classical blood tests. However, even before the publication of the 2013 guideline on the management of severe perioperative bleeding by the European Society of Anaesthesiology, ${ }^{3}$ a shift from using traditional laboratory tests to whole blood viscoelastic test was noticeable. In a landmark paper, the single-centre retrospective cohort study by Görlinger and colleagues, it was shown that rotational thromboelastometry guided transfusion was successfully implemented for patients undergoing cardiovascular surgery which led to reduced blood transfusion requirements and decreased thrombotic/thromboembolic adverse events. ${ }^{4}$ The main clinical advantage of ROTEMguided transfusion algorithms is that treatment can be initiated earlier in comparison to traditional laboratory tests. ${ }^{5}$ Besides, ROTEM-guided algorithms reduce the amount of transfusion and are associated with decreased mortality by managing blood loss in a goal-directed approach. ${ }^{6,7}$

Prospective studies of the implications of ROTEM implementation on transfusion needs and costs in cardiac surgery are scarce. Haas and colleagues published a systematic review, of which only four studies had a prospective design. ${ }^{8}$ Of these four prospective studies, one was in 100 paediatric patients and the three other studies were randomized controlled trials in 100, 56, and 61 patients, respectively. Since the publication of Haas, several other publications 


\section{Chapter 7}

were made available, ${ }^{9}$ but no large prospective cohort investigating the daily routine before and after implementation of ROTEM-guided transfusion algorithms has been published.

Our haemostasis registry of patients in cardiac surgery (HEROES-CS) addressed changes in outcome of surgery before and after ROTEM implementation for guiding transfusion. The study was designed to gather all relevant information on pre-, intra- and postoperative variables of cardiac surgery patients which could explain transfusion demand of allogeneic blood products and other procoagulant medication in a single center design. From the resulting database, we extracted patient and procedure characteristics and calculated transfusion needs, adverse bleeding events, and eventually potential costs and/or savings associated with the implementation of ROTEM diagnostics.

Based on previous studies, our hypothesis was that ROTEM-guided transfusion algorithms will reduce transfusion needs of allogeneic blood products, might increase the use of prohaemostatic medication, and will potentially reduce adverse bleeding events. Overall, we expected that ROTEM implementation would produce significant cost savings in cardiac surgery patients.

\section{Materials and Methods}

\section{Study setting and ethics}

The HEROES-CS database is a prospective observational non-management study of patients undergoing cardiac surgery. Patient, operation, laboratory, and transfusion characteristics were collected during two consecutive periods of three months in a single tertiary university hospital in Maastricht, The Netherlands. All data acquisition was done by members of the team, not directly involved in the management of the patients. Around one thousand cardiac surgery operations are performed yearly in our hospital. ${ }^{10}$ In the first period prospective data was collected from patients operated from the $10^{\text {th }}$ of October, 2011 to the $13^{\text {th }}$ of January, 2012. A second period recruited patients from the $25^{\text {th }}$ of February, 2014 up to the $8^{\text {th }}$ of July, 2014 having a one month break of inclusions in April 2014. Follow-up was 30 days from the day of operation or until discharge from the hospital. If a patient was discharged within 30 days of 
follow-up, a control visit was made by telephone, which is standard practice in our hospital. The control visit is merely done for gaining information about current functional status (e.g. revalidation, mortality, etc) and patient satisfaction about their stay in our hospital. This study was approved by the local medical ethics committee (METC azM/UM).

\section{Population and operating procedures}

All patients undergoing cardiac surgery in the respective periods form part of the study cohort. Patient data were collected prospectively if surgery took place during regular operating hours. Data of operations outside regular operating hours were collected retrospectively the day after. There were no exclusion criteria for this study.

Antithrombotic and antiplatelet medication was withheld/bridged in all cases, except for acetylsalicylic acid, which was continued. On arrival at the operating theatre, standard monitoring was applied. Patients received a large bore IV access and an arterial line. Induction of anaesthesia was done according to hospital protocol. After intubation, a central venous line was placed preferably in the right internal jugular vein. Maintenance was done using continuous total intravenous anaesthesia with propofol and an opioid. Activated clotting times $(A C T)$ were analysed using a point-of-care (POC) device before initiation of the extracorporeal circulation (ECC), if applicable. Heparin was administered in a dose of $300 \mathrm{IU} / \mathrm{kg}$ of body weight (Heparin Leo, Leo Pharmaceutical Products BV, Weesp, The Netherlands) aiming for an ACT >400 seconds before ECC initiation. Haematocrit was kept above $0.23 \mathrm{~L} / \mathrm{L}$ (this equals to a haemoglobin of around $7 \mathrm{~g} / \mathrm{dL}^{11}$ ) when on ECC under normothermic conditions. Heparin was reversed with protamine hydrochloride (Valeant Pharmaceuticals, Eschborn, Germany) in a 1:1 ratio of the loading dose. An extra protamine dose could be given if the ACT was above $10 \%$ of the baseline value. At the end of surgery all patients were transferred sedated and intubated to the ICU. At arrival on the ICU transfusion algorithms were initiated. In non-bleeding patients $100 \mathrm{mg}$ acetylsalicylic acid was given intravenously at six hours after arrival on the ICU, if indicated. 


\section{Transfusion algorithms}

Patients in the first cohort were treated according to standard laboratory results on the discretion of the attending clinicians (figure 7.1). During surgery and on arrival at the ICU all patients were treated for hypothermia ( $<35$ degrees Celsius), anaemia (haematocrit $<0.23 \mathrm{~L} / \mathrm{L}$ ), acidosis ( $\mathrm{pH}<7.25$ ), and hypocalcaemia (below normal) first (primary conditions for adequate haemostasis). For all patients $\mathrm{pH}$, temperature, calcium level, haematocrit, thrombocyte counts, fibrinogen level, prothrombin time as internal normalized ratio (INR), and activated partial thromboplastin time (aPTT) were measured. Bleeding accompanied by a haematocrit below $0.23 \mathrm{~L} / \mathrm{L}$ was used as a red blood cell transfusion trigger. Thrombocyte concentrates were given if thrombocyte counts fell below 80 $x 10^{9} / L$ or bleeding was deemed due to thrombocyte dysfunction. DDAVP could be given for suspected thrombocyte dysfunction in case of adequate thrombocyte counts. Prolonged PT, prolonged INR or low fibrinogen levels were used as a trigger for fresh frozen plasma transfusion or prothrombin complex concentrate. Low fibrinogen could be treated by fibrinogen concentrate when PT or INR were normal. Protamine sulphate was given for prolonged aPTT's in suspicion of remaining heparin effect.

Patients in the second cohort were transfused following the thromboelastometry based transfusion algorithms (figure 7.2). Again, during surgery and on arrival at the ICU all patients were treated for hypothermia ( $<35$ degrees Celsius), anaemia (haematocrit $<0.23 \mathrm{~L} / \mathrm{L}$ ), acidosis $(\mathrm{pH}<7.25$ ), and hypocalcaemia (below normal) first (primary conditions for adequate haemostasis). ROTEM analysis could be performed during surgery as judged by the operating team of surgeons, anaesthesiologists, and perfusionists. ROTEM analysis was done in every patient at the ICU, using the following guidelines:

- First the primary conditions of adequate haemostasis in each patient were optimized.

- If no bleeding was observed from the chest tubes $(>100 \mathrm{~mL}$ in the first hour), no further treatment was required. 


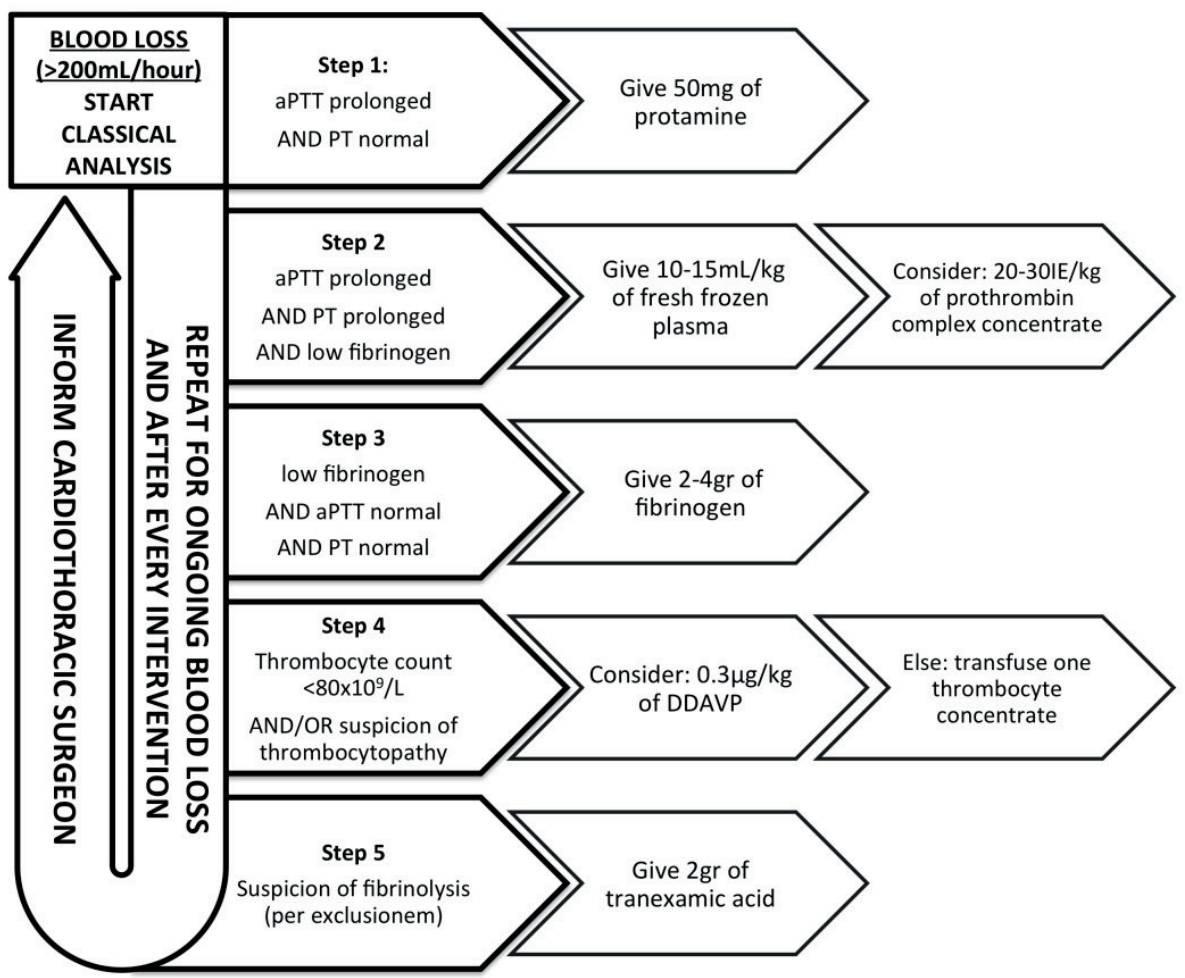

Figure 7.1 Classical-guided transfusion algorithm for postoperative blood loss more than $200 \mathrm{~mL}$ per hour.

In the first step residual heparin is assessed by aPTT and if evident, protamine is given as an antidote. The second step detects deficiencies in circulating coagulation proteins as evidenced by prolonged clotting times on aPTT and PT and by low fibrinogen. The advice was to transfuse fresh frozen plasma. Prothrombin complex concentrate can be given as an alternative in case of normal fibrinogen levels or together with fibrinogen concentrate. In step three the amount of fibrinogen is investigated. In isolated hypofibrinogenemia, fibrinogen is given as a concentrate. In the fourth step the contribution of the thrombocytes for coagulation is determined. First DDAVP can be considered to improve thrombocyte function. If DDAVP was not given thrombocyte transfusion should be initatied. In the last and fifth step tranexamic acid can be given for high suspicion of fibrinolysis if all previous steps are engative. After each investigation or if blood loss is on-going the above steps need to be re-evaluated together with the primary conditions for adequate haemostasis (no anaemia, no acidosis, no hypothermia, and no hypocalcaemia). Any blood loss over $200 \mathrm{~mL}$ in the first hour and afterwards needs to be informed to the cardiac surgeon on duty.

Abbreviations: aPTT, activated partial thromboplastin time; DDAVP, 1-desamino-8-D-arginine vasopressin; PT, prthrombin time

- If drainage was $100-200 \mathrm{~mL}$ in the first hour; 2 gram of tranexamic acid was given together with $50 \mathrm{mg}$ protamine sulphate (if evidence for circulating heparin was present on ROTEM analysis in step 1 of figure 7.2). DDAVP was given at a dose of 0.3 microgram $/ \mathrm{kg}$ if there was high suspicion of (acquired) von Willebrand disease or a suspected thrombocyte dysfunction. 


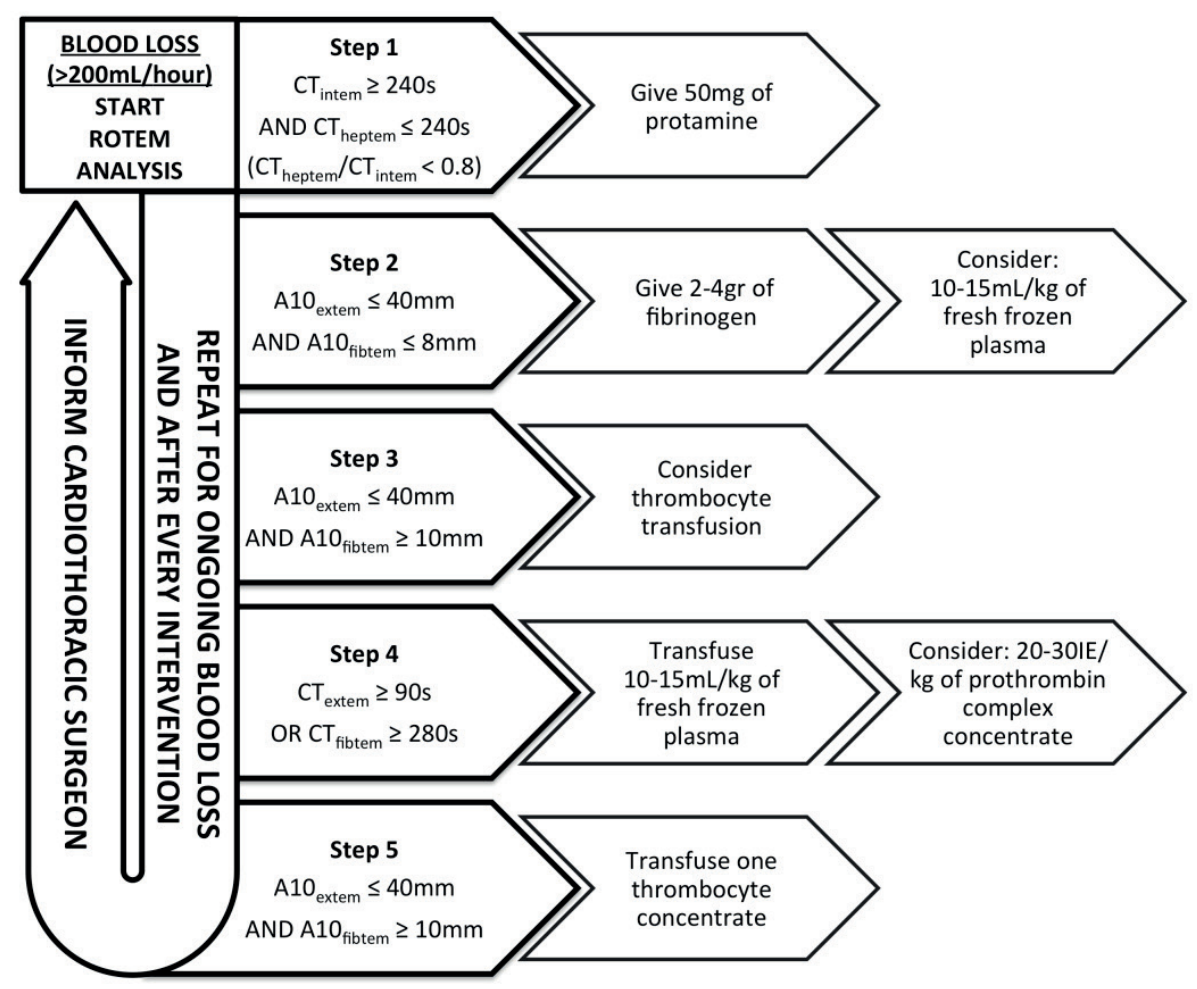

Figure 7.2 ROTEM-guided transfusion algorithm for postoperative blood loss more than $200 \mathrm{~mL}$ per hour.

In the first step residual heparin is assessed and if evident, protamine is given as an antidote.

In step two the amount of fibrinogen is investigated. If it is too low, fibrinogen is given as concentrate. Fresh frozen plasma can be used as an alternative. In the third step the contribution of the thrombocytes for the coagulation profile is determined. If it is deemed too low thrombocyte transfusion can be considered. The fourth step detects deficiencies in circulating coagulations proteins given as prolonged clotting times in EXTEM and FIBTEM. A prolonged CT on either measurement advices to transfuse fresh frozen plasma. Prothrombin complex concentrate can be given as an alternative. In the last and fifth step transfusion of one thrombocyte concentrate is advised when the thrombocyte contribution to haemostasis is too low.

After each intervention or if blood loss is on-going the above steps need to be re-evaluated together with the primary conditions for adequate haemostasis (no anaemia, no acidosis, no hypothermia, and no hypocalcaemia). Any blood loss over $200 \mathrm{~mL}$ in the first hour and afterwards needs to be informed to the cardiac surgeon on duty.

Abbreviations: A10, amplitude at 10 minutes; CT, clotting time

- If drainage was more than $200 \mathrm{~mL}$ in the first hour or when there was continued bleeding the subsequent hours, the ROTEM algorithm was re-started

(figure 7.2). 


\section{Laboratory measurements}

ACT measurements were done point-of-care (Hemotec ACT II Automated Coagulation Timer, Medtronic Inc, Minneapolis MN, USA) at the operating theatre using native whole blood. Arterial blood gas sampling was also done point-ofcare (GEM Premier 3000, instrumentation Laboratory, Lexington MA, USA) in heparinized tubes for $\mathrm{pH}$ and calcium.

All other blood samples drawn from patients perioperatively were sent to the central diagnostic laboratory via a pneumatic tube system. ROTEM results were made available to view in real-time using a secure connection between the ROTEM devices and computers at the operating theatre and ICU. After finalizing the ROTEM analysis all results were transferred to our electronic patient management system for later review. Blood for ROTEM, aPTT, PT, and fibrinogen analysis were drawn in 3.2\% citrate tube (BD Vacutainer, Becton Dickinson, Breda, The Netherlands). For thrombocyte count blood was drawn in K2EDTA tubes (BD Vacutainer, Becton Dickinson, Breda, The Netherlands).

Thrombocyte count, haematocrit, and haemoglobin measurements were performed using a Sysmex XE-5000 analyzer (Sysmex Corporation, Kobe, Japan). Fibrinogen levels were determined using a Sysmex CA-7000 analyzer (Sysmex Corporation, Kobe, Japan) after centrifugation for 10 minutes at 2,000 g using the Clauss method. ${ }^{12}$

Thromboelastometry analysis was performed using a ROTEM-delta device (TEM Internationl GmbH, Munich, Germany) following standard protocols. Analysed ROTEM parameters were clotting time (CT), amplitude at ten minutes (A10), and maximal clot formation (MCF). These parameters were performed in EXTEM, INTEM, FIBTEM, and HEPTEM standard assays. In the EXTEM and FIBTEM assays, coagulation is extrinsically activated with the use of tissue factor. In the FIBTEM assay a platelet inhibitor (cytochalasin D) is added for a qualitative assessment of fibrinogen only. The INTEM and HEPTEM are activated via the intrinsic pathway. In the HEPTEM assay heparinase is added to eliminate any possible heparin effect on the sample. 


\section{Chapter 7}

\section{Endpoint definitions}

The following perioperative endpoints were recorded. The total amount of packed red cells, fresh frozen plasma, and thrombocyte concentrates in units per patient, which were crosschecked with our transfusion database. The amount of prohaemostatic medication given during and after the operation of 1desamino-8-D-arginine vasopressin (DDAVP) in microgram per patient, human fibrinogen concentrate (FC; Haemocomplettan $\mathrm{P}$ ) in gram per patient, recombinant activated factor VII (rFVIla; NovoSeven) in milligram per patient, protamine sulphate (PTM) in milligram per patient, prothrombin complex concentrate (PCC; Cofact) in international units per patient, calcium gluconate (CG) dose in mmol per patient, and tranexamic acid (TXA) in milligram per patient. The amount of blood loss during surgery (calculated by losses from the ECC machine and weighing of swabs) and on the ICU from chest tubes was recorded in millilitres per patient. Length of stay (LOS) in days was split up in ICU LOS and the total hospital LOS. The amount and percentage of patients needing a rethoracotomy, the number of laboratory results acquired for coagulation management per patient, as well as 30-day mortality was evaluated.

\section{Predictive variables}

The following pre-operative variables were considered for endpoint prediction and subgroup analysis. Gender, age, weight, height, BSA, logistic EuroScore ${ }^{13}$ (low mortality risk: EuroScore $<3$; medium mortality risk: EuroScore $\geq 3$ but $<6$; high mortality risk: EuroScore $\geq 6$ ), elective versus emergency surgery, type of surgery (i.e. CABG surgery, (aortic) valve surgery, combined CABG/(aortic) valve surgery, and other types), antiplatelet medication use (like COX1 (i.e. acetylsalicylic acid), P2Y12 (i.e. clopidogrel et cetera), and GP Ilbllla blockers), bridged vitamin $\mathrm{K}$ antagonist use, DOAC use, haematocrit level, thrombocyte count, INR, kidney function (estimated glomerular filtration rate (eGFR) calculated by the MDRD-equation ${ }^{14}$ ), and blood group.

The following intra-operative variables were also acquired for endpoint prediction. Length of the operative procedure, duration on ECC, duration of cross 
clamping, haematocrit level, thrombocyte count, and temperature on arrival on the ICU.

\section{Potential costs and saving}

Costs of the transfusion of blood components and prohaemostatic medication, together with those of the laboratory assessments of coagulation and costs of the secondary endpoints (rethoracotomy due to bleeding cause, ICU length of stay and non-ICU hospital length of stay) were calculated. Costs were adopted from the Dutch Healthcare Authority (Nederlandse Zorgautoriteit; NZa), which is appointed by the Dutch government for controlling the tariffs in the Dutch healthcare system.

\section{Statistical analysis}

Categorical data are presented as number and percentage, while interval data are presented by median and interquartile range (IQR) or mean [min-max]. Normally distributed data were analysed by using two-tailed unpaired Student's t-test. Continuous variables with skewed distribution were analysed using Mann-Whitney $U$ testing and dichotomous variables by means of Fisher's exact test. A $p$-value $<0.05$ was considered significant. All data analyses were done using SPSS version v23 (IBM Corp, Armonk NY, USA). Graphs were constructed using GraphPad Prism (GraphPad Prism version 5.0a for Windows, GraphPad Software, San Diego CA, USA).

\section{Results}

Complete analysis of all subjects

Three hundred fifty-five subjects were enrolled, 204 of those subjects were included in the conventional-guided transfusion group and 151 subjects in the ROTEM-guided group. Baseline characteristics (table 7.1) were not different between the two groups except for minimal differences in haematocrit $(0.42$ versus $0.41 \mathrm{~L} / \mathrm{L}, p=0.004)$ and INR (1.03 versus 0.98. $p<0.001)$, which were both lower in the ROTEM-group. The majority of patients was male (68.6 versus $74.8 \%$, respectively). A small portion (14.2 versus $17.2 \%$ ) had no antiplatelet or 
Table 7.1 Baseline characteristics

\begin{tabular}{|c|c|c|c|}
\hline Baseline characteristics & $\begin{array}{l}\text { Classical-guided } \\
\text { transfusion }(n=204)\end{array}$ & $\begin{array}{c}\text { ROTEM-guided } \\
\text { transfusion }(n=151)\end{array}$ & $p$-value \\
\hline \multicolumn{4}{|l|}{ Demographics } \\
\hline Male gender - no. (\%) & $140(68.6)$ & $113(74.8)$ & 0.236 \\
\hline Age - years (IQR) & $69(61-75)$ & $68(59-75)$ & 0.275 \\
\hline Weight - kg (IQR) & $80.0(72.0-90.0)$ & $80.0(70.0-93.4)$ & 0.804 \\
\hline Height - cm (IQR) & $172(166-178)$ & $172(166-178)$ & 0.843 \\
\hline$B S A-m^{2}(I Q R)$ & $1.95(1.81-2.10)$ & $1.96(1.81-2.14)$ & 0.962 \\
\hline \multicolumn{4}{|l|}{ Antiplatelet and -coagulant medication } \\
\hline No medication - no. (\%) & $29(14.2)$ & $26(17.2)$ & 0.461 \\
\hline Antiplatelet medication only - no. (\%) & $123(60.3)$ & $97(64.2)$ & 0.507 \\
\hline Acetylsalicylic acid only - no. (\%) & $104(84.6)$ & $83(85.6)$ & 0.852 \\
\hline Clopidogrel only - no. (\%) & $1(0.8)$ & $0(0.0)$ & 1.000 \\
\hline Dual antiplatelet medication - no. (\%) & $18(14.6)$ & $83(85.6)$ & 1.000 \\
\hline Bridged VKA only - no. (\%) & $41(20.1)$ & $22(14.6)$ & 0.207 \\
\hline Therapeutic LMWH only - no. (\%) & $2(1.0)$ & $0(0.0)$ & 0.510 \\
\hline DOAC only - no. (\%) & $0(0.0)$ & $2(1.3)$ & 0.180 \\
\hline Combined medication use - no. (\%) & $9(4.4)$ & $4(2.6)$ & 0.569 \\
\hline \multicolumn{4}{|l|}{ Clinical and laboratory characteristics } \\
\hline Haematocrit - L/L (IQR) & $0.42(0.39-0.44)$ & $0.41(0.38-0.43)$ & 0.004 \\
\hline Thrombocyte count $-\times 10^{9} / \mathrm{L}(\mathrm{IQR})^{*}$ & $231(186-278)$ & $243(206-274)$ & 0.224 \\
\hline INR - s/s (IQR) & $1.03(0.99-1.07)$ & $0.98(0.95-1.03)$ & $<0.001$ \\
\hline Blood group 0/A/B/AB - no. (\%) & $\begin{array}{c}89 / 96 / 15 / 4 \\
(43.6 / 47.1 / 7.4 / 2.0)\end{array}$ & $\begin{array}{c}65 / 73 / 10 / 3 \\
(43.0 / 48.3 / 6.6 / 2.0)\end{array}$ & 0.991 \\
\hline MDRD-eGFR - mL/min/1.73m² (IQR) & $74.6(60.3-89.5)$ & $69.9(58.7-85.6)$ & 0.064 \\
\hline Elective surgery - no. (\%) & $190(93.1)$ & $142(94.0)$ & 0.829 \\
\hline \multicolumn{4}{|l|}{ Type of surgery } \\
\hline Isolated CABG surgery - no. (\%) & $96(47.1)$ & $73(48.3)$ & 0.830 \\
\hline Off-pump CABG surgery - no. (\%) & $3(3.1)$ & $4(5.5)$ & 0.464 \\
\hline Isolated AVR surgery - no. (\%) & $44(21.6)$ & $29(19.2)$ & 0.599 \\
\hline TAVI procedure - no. (\%) & $20(45.5)$ & $10(34.5)$ & 0.467 \\
\hline Combined CABG/AVR surgery - no. (\%) & $42(20.6)$ & $26(17.2)$ & 0.496 \\
\hline Other procedures - no. (\%) & $22(10.8)$ & $23(15.2)$ & 0.259 \\
\hline Logistic EuroScore - (IQR) & $4.49(2.27-8.81)$ & $3.93(2.15-8.33)$ & 0.542 \\
\hline Low mortality risk group - no. (\%) & $43(21.1)$ & $33(21.9)$ & 0.896 \\
\hline Medium mortality risk group - no. (\%) & $85(41.7)$ & $65(43.0)$ & 0.828 \\
\hline High mortality risk group - no. (\%) & $76(37.3)$ & $53(35.1)$ & 0.738 \\
\hline
\end{tabular}

anticoagulant medication before cardiac surgery, while most subjects had only antiplatelet medication (60.3 versus $64.2 \%$ ) of which the largest proportion used solely acetylsalicylic acid (around 85\%). A small percentage of subjects underwent emergency cardiac surgery (6.9 versus 6.0\%) and half of the subjects had an isolated CABG surgery procedure. Isolated AVR surgery was done in around $20 \%$ of the subjects which made it the second largest subgroup. Logistic EuroScore did not differ between the two groups $(p=0.542)$ at a median of 4.49 for the classical-guided group versus 3.93 for the ROTEM-guided group. The 
proportions of low-medium-high risk mortality according to EuroScore was evenly distributed in both algorithm groups.

Intra- and postoperative variables are presented in table 7.2. The main findings are that the ROTEM-guided transfusion group had almost 30 minutes longer length of operation, was approximately 15 minutes longer on ECC, and had 10 minutes longer of aortic cross-clamping time. On the other hand, the ACT post ECC was higher and closer to $100 \%$ of baseline in the ROTEM-guided group. Temperature, calcium and fibrinogen levels on arrival at the ICU were not significantly different between the two groups. Thrombocyte count, though, was significantly higher in the ROTEM-guided group.

Table 7.2 Intra- and postoperative variables

\begin{tabular}{lccr}
\hline Intra-operative variables & $\begin{array}{c}\text { Classical-guided } \\
\text { transfusion }(\mathrm{n}=204)\end{array}$ & $\begin{array}{c}\text { ROTEM-guided } \\
\text { transfusion }(\mathrm{n}=151)\end{array}$ & $p$-value \\
\hline Length of operation - min (IQR) & $176(138-225)$ & $205(164-284)$ & $<0.001$ \\
ECC time - min (IQR) & $83(64-119)$ & $97(69-140)$ & 0.014 \\
Cross-clamping time - min (IQR) & $57(42-78)$ & $67(47-93)$ & 0.003 \\
ACT post ECC - \% of baseline (IQR) & $95.5(87.8-104.7)$ & $100.9(93.0-110.3)$ & 0.002 \\
\hline Postoperative variables & & & 0.929 \\
\hline Haematocrit - L/L (IQR) & $0.29(0.27-0.31)$ & $0.29(0.26-0.33)$ & $<0.001$ \\
Platelet count - $\times 10^{9} / \mathrm{L}(\mathrm{IQR})$ & $117(92-154)$ & $145(121-182)$ & 0.278 \\
Fibrinogen level - g/L (IQR) & $1.8(1.5-2.2)$ & $1.9(1.6-2.3)$ & 0.073 \\
Temperature on arrival at ICU - ${ }^{\circ} \mathrm{C}(\mathrm{IQR})$ & $36.2(35.8-36.6)$ & $36.1(35.8-36.4)$ &
\end{tabular}

Primary outcome parameters showed multiple differences between the two cohorts (table 7.3). Overall, ROTEM-guidance of transfusion was associated with less blood loss on the day of operation (880 versus $560 \mathrm{~mL}(p<0.001)$; both a decline intra- as well as postoperatively), decreased amount of cell saver return (477 versus $382 \mathrm{~mL}, p<0.001$ ), less transfusion of red blood cell concentrates (54 versus 43\%) and fresh frozen plasma (19 versus $7 \%$ ), but not of thrombocyte concentrates. Of the prohaemostatic medication, reductions in DDAVP, rFVIla, and calcium gluconate were seen, while the use of tranexamic acid and fibrinogen concentrate was significantly increased in patients undergoing the ROTEM-guided algorithm. Note that no PCC was given to any of the subjects during either period. For the secondary endpoints a tendency of reduction of the percentage of rethoracotomies (14.2 versus $10.6 \%$ ), mean ICU LOS (2.8 versus 1.8$)$, and a 30 -day mortality rate (2.5 versus $5.3 \%$ ) was seen after 
Table 7.3 Primary and secondary endpoints

\begin{tabular}{|c|c|c|c|}
\hline Primary endpoints & $\begin{array}{c}\text { Classical-guided } \\
\text { transfusion }(n=204)\end{array}$ & $\begin{array}{c}\text { ROTEM-guided } \\
\text { transfusion }(n=151)\end{array}$ & $p$-value \\
\hline Estimated blood loss day one - $\mathrm{mL}(\mathrm{IQR})$ & $880(600-1292)$ & $560(371-810)$ & $<0.001$ \\
\hline Intra-operative - mL (IQR) & $150(50-250)$ & $200(100-358)$ & 0.003 \\
\hline Postoperative - $\mathrm{mL}$ (IQR) & $670(460-1093)$ & $350(230-533)$ & $<0.001$ \\
\hline $1^{\text {st }}$ hour - mL (IQR) & $80(44-130)$ & $60(20-110)$ & $<0.001$ \\
\hline $2^{\text {nd }}$ hour - $\mathrm{mL}$ (IQR) & $70(40-120)$ & $50(20-95)$ & $<0.001$ \\
\hline Cell saver autologous return - $\mathrm{mL}(\mathrm{IQR})$ & $477(381-658)$ & $382(250-600)$ & $<0.001$ \\
\hline \multicolumn{4}{|l|}{ Allogeneic transfusion products ${ }^{*}$} \\
\hline Patients that received RBC - no. (\%) & $111(54.4)$ & $65(43.0)$ & 0.041 \\
\hline On day of operation - units & $0(0-2) ; 1.8[0-22]$ & $0(0-1) ; 0.7[0-8]$ & $<0.001$ \\
\hline Intra-operative - units & $0(0-0) ; 0.8[0-15]$ & $0(0-0) ; 0.4[0-8]$ & 0.097 \\
\hline Patients that received FFP - no. (\%) & $38(18.6)$ & $11(7.3)$ & 0.003 \\
\hline On day of operation - units & $0(0-0) ; 0.8[0-1]$ & $0(0-0) ; 0.3[0-6]$ & 0.007 \\
\hline Intra-operative - units & 0 (0-0); $0.4[0-9]$ & $0(0-0) ; 0.2[0-6]$ & 0.062 \\
\hline Patients that received TC - no. (\%) & $51(25.0)$ & $27(17.9)$ & 0.121 \\
\hline On day of operation - units & $0(0-0) ; 0.4[0-6]$ & $0(0-0) ; 0.2[0-3]$ & 0.103 \\
\hline Intra-operative - units & $0(0-0) ; 0.2[0-3]$ & $0(0-0) ; 0.2[0-3]$ & 0.489 \\
\hline \multicolumn{4}{|l|}{ Prohaemostatic medication $^{*}$} \\
\hline Patients that received TXA - no. (\%) & $174(85.3)$ & $147(97.4)$ & $<0.001$ \\
\hline On day of operation - g & $3(3-3) ; 2.9[0-6]$ & $3(3-3) ; 2.8[0-5]$ & 0.145 \\
\hline Intra-operative - $\mathrm{g}$ & 3 (3-3); $2.5[0-5]$ & 3 (3-3); 2.8 [0-5] & 0.179 \\
\hline Patients that received PTM - no. (\%) & $188(92.2)$ & $142(94.0)$ & 0.536 \\
\hline On day of operation - mg & $\begin{array}{c}250(250-300) \\
253.4[0-700]\end{array}$ & $\begin{array}{c}250(200-300) \\
249.7[0-500]\end{array}$ & 0.463 \\
\hline Intra-operative - mg & $\begin{array}{c}250(250-300) \\
253.1[0-700]\end{array}$ & $\begin{array}{c}250(200-300) ; \\
249.7[0-500]\end{array}$ & 0.469 \\
\hline Patients that received DDAVP - no. (\%) & $40(19.6)$ & $1(0.7)$ & $<0.001$ \\
\hline On day of operation - $\mu \mathrm{g}$ & $0(0-0) ; 4.8[0-36]$ & $0(0-0) ; 0.2[0-30]$ & $<0.001$ \\
\hline Intra-operative - $\mu \mathrm{g}$ & $0(0-0) ; 0.5[0-30]$ & $0(0-0) ; 0[0-0]$ & 0.084 \\
\hline Patients that received FC - no. (\%) & $18(8.8)$ & $29(19.2)$ & 0.007 \\
\hline On day of operation - $\mathrm{g}$ & $0(0-0) ; 0.3[0-8]$ & $0(0-0) ; 0.5[0-4]$ & 0.006 \\
\hline Intra-operative - $\mathrm{g}$ & $0(0-0) ; 0.1[0-8]$ & $0(0-0) ; 0.40-4]$ & $<0.001$ \\
\hline Patients that received rFVIIa - no. (\%) & $2(1.0)$ & $0(0.0)$ & 0.510 \\
\hline On day of operation - mg & $0(0-0) ; 0.1[0-10]$ & $0(0-0) ; 0.0[0-0]$ & 0.223 \\
\hline Intra-operative - mg & $0(0-0) ; 0.05[0-10]$ & $0(0-0) ; 0.0[0-0]$ & 0.390 \\
\hline Patients that received CG - no. (\%) & $32(15.7)$ & $12(7.9)$ & 0.034 \\
\hline On day of operation - mmol & $0(0-0) ; 0.9[0-27.6]$ & $0(0-0) ; 0.2[0-9.2]$ & 0.003 \\
\hline Intra-operative - mmol & $0(0-0) ; 0.6[0-20.7]$ & $0(0-0) ; 0.1[0-6.9]$ & 0.005 \\
\hline \multicolumn{4}{|l|}{ Secondary endpoints ${ }^{*}$} \\
\hline Rethoracotomy - no. (\%) & $29(14.2$ & $16(10.6)$ & 0.337 \\
\hline Due to bleeding - no. (\%) ${ }^{* *}$ & $21(72.4)$ & $11(68.8)$ & 1.000 \\
\hline ICU length of stay - days & $1(1-2) ; 2.8[0-65]$ & $1(1-2) ; 1.8[0-28]$ & 0.826 \\
\hline Hospital length of stay - days & $9(7-14) ; 12.9$ [0-81] & $7(6-10) ; 9.7[1-60]$ & $<0.001$ \\
\hline 30-day mortality - no. (\%) & $5(2.5)$ & $8(5.3)$ & 0.252 \\
\hline Lost-to-follow-up - no. (\%) & $10(4.9)$ & $18(11.9)$ & 0.017 \\
\hline
\end{tabular}

${ }^{*}$ Results are median (IQR); mean [min-max]

" Composite endpoint of rethoracotomy for tamponade, thorax drain bleeding, or mixed reasons

ROTEM implementation. However, none of these differences were statistically significant. Hospital LOS was reduced by a mean of 3 days after ROTEM implementation $(p<0.001)$. Of 10 (classical) and 18 (ROTEM) subjects we could not determine if they were still alive after 30 days, because no call was an- 
swered for the control visit by telephone $(p=0.017)$. Median EuroScore as a predictor of mortality was higher in deceased patients than in patients alive or in patients with unknown status (figure 7.3). Finally, potential savings (based on the mean difference per patient) was more than 3,400 Euro per patient after implementation of ROTEM-guided transfusion protocols (table 7.4).

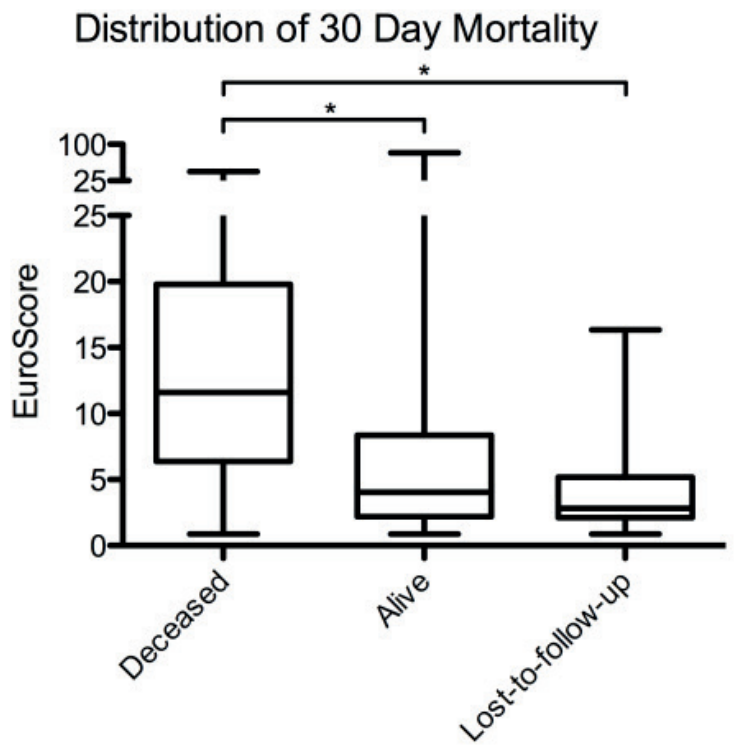

Figure 7.3 Distribution of EuroScore among mortality status at 30 days

Median EuroScore was 11.61 for deceased patients, 4.04 for patients still alive at 30 days followup, and 2.83 for patients lost-to-follow-up.

${ }^{*} p<0.01$

Isolated CABG surgery subgroup

Similarly, an analysis of the isolated CABG surgery group was made (supplement, tables S1 to S4), which was the largest subgroup with the most standardized operating procedures.

In total 96 and 73 subjects had isolated CABG surgery in the classical and ROTEM-group, respectively. Overall no major differences were found between the two groups of the baseline characteristics, besides more use of nothing but antiplatelet medication in the ROTEM-group (78 versus 90\% use, $p=0.039$ ) and again lower INR $(1.02$ versus $0.97, p<0.001)$ in the ROTEM-group. Compared to the overall group, male gender was more predominant $(82 \%)$, but age 
Table 7.4 Estimation of costs and potential savings due to implementation of a ROTEM-guided algorithm

\begin{tabular}{lrrr}
\hline Costs - Euro per patient & $\begin{array}{c}\text { Classical-guided } \\
\text { transfusion }\end{array}$ & $\begin{array}{c}\text { ROTEM-guided } \\
\text { transfusion }\end{array}$ & $\begin{array}{c}\text { Potential } \\
\text { savings }\end{array}$ \\
\hline Allogeneic transfusion products - total & & & 445.62 \\
Red blood cell concentrate & 570.46 & 341.01 & 229.45 \\
Fresh frozen plasma & 190.14 & 75.69 & 114.45 \\
$\quad$ Thrombocyte concentrate & 254.30 & 152.58 & 101.72 \\
\hline Prohaemostatic medication - total & & & 20.48 \\
$\quad$ Tranexamic acid & 18.74 & 18.01 & 0.73 \\
Protamine & 0.04 & 0.04 & 0.00 \\
DDAVP & 9.00 & 0.33 & 8.67 \\
Fibrinogen concentrate & 137.50 & 189.13 & -51.63 \\
Activated recombinant factor VII & 62.52 & 0.00 & 62.52 \\
Calcium gluconate & 0.29 & 0.10 & 0.19 \\
\hline Laboratory tests - total & & & -68.81 \\
ROTEM & 20.53 & 97.98 & -77.45 \\
Activated partial thromboplastin time & 8.75 & 6.27 & 2.48 \\
Prothrombin time as INR & 3.51 & 1.63 & 1.88 \\
Fibrinogen level & 13.98 & 11.80 & 2.18 \\
$\quad$ Thrombocyte count & 18.05 & 15.95 & 2.10 \\
\hline Secondary endpoints - total & & & 3039.41 \\
Rethoracotomy for bleeding cause* & 809.17 & 572.62 & 236.55 \\
ICU length of stay & 6195.79 & 4022.77 & 2173.02 \\
$\quad$ Non ICU hospital length of stay & 2890.11 & 2260.27 & 629.84 \\
\hline Grand total & & & 3436.70 \\
\hline
\end{tabular}

"Based of means of each cohort and the current NZa tariffs

* Composite endpoint of rethoracotomy for tamponade, thorax drain bleeding, or mixed reasons

was similar. Other clinical and laboratory characteristics had comparable trends to the overall group, but logistic EuroScore was lower (2.65-2.85 in the isolated CABG surgery group compared to 3.93-4.49 in the overall group).

For the intra-operative variables the differences between classical-guided and ROTEM-guided revealed to be similar to the complete set of subjects. Lengths of operation, of ECC, and of cross-clamping were all longer in the ROTEMgroup. Likewise was the change of the postoperative variables on the ICU after ROTEM implementation.

Regarding the primary endpoints in the isolated CABG subgroup: the ROTEMgroup had less blood loss mainly postoperatively, less transfusion of RBC on the day of operation, less transfusion of FFP during the study period, but increased use of fibrinogen concentrate (which was not used at all in the classical-guided group). The use of DDAVP was diminished and rVFlla was not used in any of the two groups. For the secondary endpoints only a reduction in the incidence of rethoracotomy was observed (absolute risk reduction 8.8\%, $p=$ 
0.042) irrespective of cause. Again, lost-to-follow-up was higher in the ROTEMgroup compared to the classical group (5.2 versus $15.1 \%, p=0.036$ ).

In this isolated CABG subgroup potential savings due to ROTEM implementation were approximately 4,500 Euro per patient

\section{High EuroScore subgroup}

In order to reveal what the impact of the adoption of the new ROTEM-guided transfusion protocol is on outcome parameters, we looked at another subgroup of subjects, mainly those who are prone to high consumption of blood products. Multivariate analysis with the pre-operative variables as predictors for the total consumption of blood products (total number of red blood cell, fresh frozen plasma and thrombocyte concentrate transfusion combined) showed that EuroScore would correlate with transfusion the best $\left(\beta=0.297, p<0.001\left(r^{2}=\right.\right.$ 0.090; F-ratio: $32.2(p<0.001))$. In figure 7.4 the distribution among the three EuroScore groups (low-medium-high) and their protocol subdivision is shown for each blood product. Subjects with higher EuroScores were prone to more transfusion of red blood cells, fresh frozen plasma, and thrombocyte concentrates. This is evident for both subgroups according to transfusion algorithm. The high EuroScore group was thus chosen to look into more thoroughly (supplement, tables S5 to S8).

In total 129 subjects (76 classical- versus 53 ROTEM-guided subjects) fell into the high mortality risk group with a EuroScore more than 6. Once again, INR was lower in the ROTEM-guided group and sole use of acetylsalicylic acid was higher in the ROTEM-group. Dual antiplatelet therapy on the other hand was lower in the ROTEM group (35 versus 10\%, $p=0.023$ ).

Intra-operative variables were all higher $(p<0.05)$ in the ROTEM-group: median length of operation by 1.5 hours, median ECC time by 45 minutes, median cross-clamping time by 20 minutes, and median ACT by $7 \%$. Postoperative values of haematocrit, thrombocyte count, fibrinogen level, and temperature were not different between the two groups.

Allogeneic blood products were equally distributed between the two groups. DDAVP was again reduced almost to zero after ROTEM implementation, while 
the use of tranexamic acid, protamine, and fibrinogen concentrate use was elevated in this high mortality risk subgroup. Hospital length of stay was reduced by 3 days in the ROTEM group, all other secondary endpoints were equal between the two groups.

For this high EuroScore subgroup ROTEM implementation could save more than 2,300 Euro per patient.
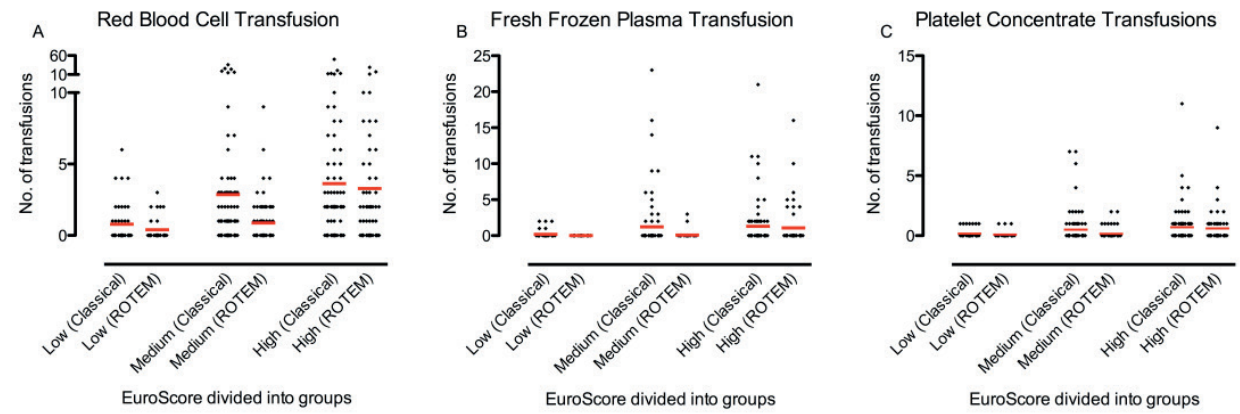

Figure 7.4 Distribution of blood product use among EuroScore groups and their protocol subdivisions.

Shown is the amount of transfusions each patient (black diamonds) had for the three blood products $(\mathrm{a}-\mathrm{c})$. Each transfusion product is subdivided by the EuroScore of each patient in three classes: low mortality risk (EuroScore $<3$ ), medium mortality risk (EuroScore $\geq 3$ but $<6$ ), and high mortality risk (EuroScore $\geq 6$ ). Lastly the graphs were split up by the two cohorts (classical-guided versus ROTEM-guided algorithm). The red bars represent the mean number of transfusions for all patients in their respective lane.

\section{Discussion}

The main consequence of the introduction of a ROTEM-guided transfusion protocol for cardiac surgery bleeding management appears to be a significant reduction of blood loss perioperatively, as well as a lesser need for transfusion of allogeneic red blood cells and fresh frozen plasma, in our hospital. On the other hand we noticed an increase in the use of tranexamic acid and especially an increase in fibrinogen concentrate as a more condensed alternative source of fibrinogen in comparison to fresh frozen plasma.

Meanwhile the use of DDAVP and activated recombinant factor VII was almost abolished. The use of thrombocyte concentrates did not seem to have changed. Secondary endpoints did not seem to be impacted by the introduction of a ROTEM-guided algorithm except for shorter median and mean hospital length 
of stay. However, because the costs of the secondary endpoints outweigh those of the transfusion products, prohaemostatic medication, and the laboratory tests, potential cost saving could be substantial at an estimated 3.4 million Euro per year for our hospital situation with around 1000 procedures annually.

Although the two groups were observed two years apart, baseline characteristics did not differ. The only difference was seen in haematocrit level and INR, which were both lower in the ROTEM-guided group. We think that the clinical importance of this significant difference is negligible, because neither would need an intervention. The first important differences seem to be apparent during and after the operation. The intra- and postoperative variables in the ROTEMguided group suggest a higher risk of bleeding in comparison to the classicalguided group; longer operating times, longer ECC times, and longer crossclamping times are risk factors for the occurrence of bleeding and use of blood products thereafter. ${ }^{15-18}$ On the contrary, the higher thrombocyte counts on arrival on the ICU would be more advantageous for the ROTEM-guided group. However, high thrombocyte counts do not indicate adequate thrombocyte function. Overall, baseline characteristics do not indicate a difference between the two groups in potential transfusion need, but in the end, less transfusion products were administered to the ROTEM-group, despite having the higher risk for transfusion according to the intra-operative variables.

In the present study, the number of rethoracotomies due to bleeding was basically unchanged $(10.3 \%$ in the classical group versus $7.3 \%$ in the ROTEM group, $p=0.355)$, which was still somewhat higher than expected from literature. ${ }^{18-20}$ In the Dutch DECS trial reexploration because of bleeding was done in $6.8 \%$ of the patients. ${ }^{19}$ This amount was even lower $(5.7 \%)$ in the group without pre-emptive dexamethasone, which has been the common practice in our hospital. Re-exploration due to bleeding was seen in $3.6 \%$ of the patients in one other study, in which the authors also demonstrated that prolonged ECC time (especially over 150 minutes) was a risk factor for bleeding. ${ }^{20}$ Another study had a similar percentage of $3.6 \%$ of reexplorations for bleeding post-surgery. ${ }^{18}$ They found that prolonged ECC and cross-clamping times resulted in more coagulopathic bleeding. In our study the ROTEM-guided transfusion group had longer 


\section{Chapter 7}

ECC and cross-clamping times compared to the classical-guided transfusion group, which did not exert to more rethoracotomies because of bleeding. Coagulopathic bleeding might be more efficiently diagnosed and managed using ROTEM results, such that normal coagulation profiles in bleeding patients would necessitate surgical intervention.

Consequent monitoring of haemostasis results in earlier interventions with use of transfusion, medication, and/or surgical means. ROTEM-guided treatment of perioperative bleeding can be initiated up to 25 minutes sooner than when using traditional laboratory tests. ${ }^{5}$ Consequently, less blood is shed and less dilutional coagulopathy might occur due to continued infusion of crystalloids and/or colloids in a bleeding patient while waiting for the test results.

Mortality data are comparable to a previous report of cardiac procedures performed from 2007 to 2010 in the Netherlands. ${ }^{10}$ Our 30-day mortality rate of $3.7 \%$ ( 13 out of 355 ) is similar to the reported $3.0 \%$ in this study by Siregar et al. The calculated EuroScore seems to reflect the same findings: median logistic EuroScore was 4.0 in the database of the Netherlands Association for CardioThoracic Surgery, while in our HEROES-CS database the median logistic EuroScore was 4.49 in the classical group and 3.93 in the ROTEM group. Other distributions of our patient and clinical characteristics are in line with the national results: around $70 \%$ is of male gender, average age is between $65-70$ years old, half of the patients are in need of isolated CABG surgery, and only $5 \%$ of surgeries are emergency procedures. Mean hospital length of stay was 8.7 days (or 12.4 days when the extended length of stay in the referring hospital was included) in the national database. We have shown that mean hospital length of stay was reduced after implementation of ROTEM-guided transfusion algorithms from 12.9 days to 9.7 days. This all leads to our conclusion that our HEROES-CS database is a good representative subset of cardiac surgery practice in the Netherlands.

Our subgroups analysis of isolated CABG surgery showed lower EuroScores (2.85 and 2.65 for each transfusion group) in comparison to national figures (averages were 4.9 in 2011 and 4.7 in 2014 for isolated CABG surgery). ${ }^{21}$ In our high mortality risk subgroup (EuroScore $\geq 6$ ), which are more prone to the need 
for transfusion, ROTEM-guidance resulted in less blood loss and use of cell saver return of autologous blood. Tranexamic acid and fibrinogen concentrate were given more in the ROTEM group, while the allogeneic blood products were distributed evenly between the two groups. This is reflected in the potential cost savings, which are lower for high risk cardiac surgery when implementing ROTEM-guided transfusion protocols in comparison to the isolated CABG surgery subgroup. Cost-effectiveness is maintained due to shorter hospital length of stay in this subgroup of large consumption of transfusion products.

One of the effects that could have introduced bias and explain the impact of these new ROTEM-guided protocols is that clinicians need to get used to them. Classical-guided transfusion algorithms are well known and do not need rechecking of the protocol each time bleeding occurs. New protocols and guidelines need to be familiarized with and are consulted more frequently. This improves adherence and could therefore explain our positive findings. Also, information bias might have occurred in both groups due to missing data after discharge from our hospital to the referral hospital. We have made 30-day control telephone follow-ups in all subjects, but we did not retrieve information from all of them. This could mean that the subjects had died, but we do not know this for sure; in these cases subjects were lost-to-follow-up. However, of all control visits no patient had died when gaining this information from the spouse. Besides, judging from figure 7.3 we do not expect that patients have died after discharge during the remainder of the 30-days follow-up. Missing data on transfusions after discharge are present, because control visits had no questions on transfusions. However, asking subjects about unrecorded transfusions might introduce recall bias.

In conclusion, ROTEM-guided transfusion algorithms have reduced transfusion needs of allogeneic blood products, has indeed increased the use of prohaemostatic medication, and has reduced adverse bleeding events. Overall, ROTEM implementation has the potential to save costs in cardiac surgery patients. 


\section{Chapter 7}

\section{References}

1. Bolton-Maggs $\mathrm{PH}$, Cohen $\mathrm{H}$. Serious Hazards of Transfusion (SHOT) haemovigilance and progress is improving transfusion safety. Br J Haematol. 2013 Nov;163(3):303-14.

2. Carson JL, Grossman BJ, Kleinman S, Tinmouth AT, Marques MB, Fung MK, Holcomb JB, Illoh O, Kaplan LJ, Katz LM, Rao SV, Roback JD, Shander A, Tobian AA, Weinstein R, Swinton McLaughlin LG, Djulbegovic B; Clinical Transfusion Medicine Committee of the AABB. Red blood cell transfusion: a clinical practice guideline from the AABB*. Ann Intern Med. $2012 \mathrm{Jul}$ 3;157(1):49-58.

3. Kozek-Langenecker SA, Afshari A, Albaladejo P, Santullano CA, De Robertis E, Filipescu DC, Fries D, Görlinger K, Haas T, Imberger G, Jacob M, Lancé M, Llau J, Mallett S, Meier J, RaheMeyer N, Samama CM, Smith A, Solomon C, Van der Linden P, Wikkelsø AJ, Wouters P, Wyffels P. Management of severe perioperative bleeding: guidelines from the European Society of Anaesthesiology. Eur J Anaesthesiol. 2013 Jun;30(6):270-382.

4. Görlinger K, Dirkmann D, Hanke AA, Kamler M, Kottenberg E, Thielmann M, Jakob H, Peters J. First-line therapy with coagulation factor concentrates combined with point-of-care coagulation testing is associated with decreased allogeneic blood transfusion in cardiovascular surgery: a retrospective, single-center cohort study. Anesthesiology. 2011 Dec;115(6):1179-91.

5. Olde Engberink RH, Kuiper GJ, Wetzels RJ, Nelemans PJ, Lance MD, Beckers EA, Henskens YM. Rapid and correct prediction of thrombocytopenia and hypofibrinogenemia with rotational thromboelastometry in cardiac surgery. J Cardiothorac Vasc Anesth. 2014 Apr;28(2):210-6.

6. Wikkelsø A, Wetterslev J, Møller AM, Afshari A. Thromboelastography (TEG) or rotational thromboelastometry (ROTEM) to monitor haemostatic treatment in bleeding patients: a systematic review with meta-analysis and trial sequential analysis. Anaesthesia. 2017 Apr;72(4):519531.

7. Whiting P, Al M, Westwood M, Ramos IC, Ryder S, Armstrong N, Misso K, Ross J, Severens J, Kleijnen J. Viscoelastic point-of-care testing to assist with the diagnosis, management and monitoring of haemostasis: a systematic review and cost-effectiveness analysis. Health Technol Assess. $2015 \mathrm{Jul}$;19(58):1-228, v-vi.

8. Haas T, Görlinger K, Grassetto A, Agostini V, Simioni P, Nardi G, Ranucci M. Thromboelastometry for guiding bleeding management of the critically ill patient: a systematic review of the literature. Minerva Anestesiol. 2014 Dec;80(12):1320-35.

9. Nakayama Y, Nakajima Y, Tanaka KA, Sessler DI, Maeda S, lida J, Ogawa S, Mizobe T. Thromboelastometry-guided intraoperative haemostatic management reduces bleeding and red cell transfusion after paediatric cardiac surgery. Br J Anaesth. 2015 Jan;114(1):91-102.

10. Siregar S, Groenwold RH, Versteegh MI, Takkenberg JJ, Bots ML, van der Graaf Y, van Herwerden LA. Data Resource Profile: adult cardiac surgery database of the Netherlands Association for Cardio-Thoracic Surgery. Int J Epidemiol. 2013 Feb;42(1):142-9. 
11. Society of Thoracic Surgeons Blood Conservation Guideline Task Force, Ferraris VA, Brown JR, Despotis GJ, Hammon JW, Reece TB, Saha SP, Song HK, Clough ER; Society of Cardiovascular Anesthesiologists Special Task Force on Blood Transfusion, Shore-Lesserson LJ, Goodnough LT, Mazer CD, Shander A, Stafford-Smith M, Waters J; International Consortium for Evidence Based Perfusion, Baker RA, Dickinson TA, FitzGerald DJ, Likosky DS, Shann KG. 2011 update to the Society of Thoracic Surgeons and the Society of Cardiovascular Anesthesiologists blood conservation clinical practice guidelines. Ann Thorac Surg. 2011 Mar;91(3):94482.

12. Clauss. [Rapid physiological coagulation method in determination of fibrinogen]. Acta Haematol. 1957 Apr;17(4):237-46.

13. Roques F, Michel P, Goldstone AR, Nashef SA. The logistic EuroSCORE. Eur Heart J. 2003 May;24(9):881-2.

14. Levey AS, Bosch JP, Lewis JB, Greene T, Rogers N, Roth D. A more accurate method to estimate glomerular filtration rate from serum creatinine: a new prediction equation. Modification of Diet in Renal Disease Study Group. Ann Intern Med. 1999 Mar 16;130(6):461-70.

15. Surgenor SD, Kramer RS, Olmstead EM, Ross CS, Sellke FW, Likosky DS, Marrin CA, Helm RE Jr, Leavitt BJ, Morton JR, Charlesworth DC, Clough RA, Hernandez F, Frumiento C, Benak A, DioData C, O'Connor GT; Northern New England Cardiovascular Disease Study Group. The association of perioperative red blood cell transfusions and decreased long-term survival after cardiac surgery. Anesth Analg. 2009 Jun;108(6):1741-6.

16. Salis S, Mazzanti VV, Merli G, Salvi L, Tedesco CC, Veglia F, Sisillo E. Cardiopulmonary bypass duration is an independent predictor of morbidity and mortality after cardiac surgery. J Cardiothorac Vasc Anesth. 2008 Dec;22(6):814-22.

17. Nissinen J, Biancari F, Wistbacka JO, Peltola T, Loponen P, Tarkiainen P, Virkkilä M, Tarkka M. Safe time limits of aortic cross-clamping and cardiopulmonary bypass in adult cardiac surgery. Perfusion. 2009 Sep;24(5):297-305.

18. Hall TS, Sines JC, Spotnitz AJ. Hemorrhage related reexploration following open heart surgery: the impact of pre-operative and post-operative coagulation testing. Cardiovasc Surg. 2002 Apr;10(2):146-53.

19. Van Osch D, Dieleman JM, Nathoe HM, Boasson MP, Kluin J, Bunge JJ, Nierich AP, Rosseel PM, van der Maaten JM, Hofland J, Diephuis JC, de Lange F, Boer C, van Dijk D; Dexamethasone for Cardiac Surgery Study Group. Intraoperative High-Dose Dexamethasone in Cardiac Surgery and the Risk of Rethoracotomy. Ann Thorac Surg. 2015 Dec;100(6):2237-42.

20. Dacey LJ, Munoz JJ, Baribeau YR, Johnson ER, Lahey SJ, Leavitt BJ, Quinn RD, Nugent WC, Birkmeyer JD, O'Connor GT. Reexploration for hemorrhage following coronary artery bypass grafting: incidence and risk factors. Northern New England Cardiovascular Disease Study Group. Arch Surg. 1998 Apr;133(4):442-7.

21. Transparant over uitkomsten van hartchirurgie (1) De sterfte in Nederland na hartchirurgie over de periode 2007-2015. Accessed on December 21st 2016 at http://www.nvtnet.nl/index.asp?page_id=126 
Supplement

Table S1 Baseline characteristics of the isolated CABG surgery subgroup

\begin{tabular}{|c|c|c|c|}
\hline Baseline characteristics & $\begin{array}{l}\text { Classical-guided } \\
\text { transfusion }(n=96)\end{array}$ & $\begin{array}{l}\text { ROTEM-guided } \\
\text { transfusion }(n=73)\end{array}$ & $p$-value \\
\hline \multicolumn{4}{|l|}{ Demographics } \\
\hline Male gender - no. (\%) & $79(82.3)$ & $60(82.2)$ & 1.000 \\
\hline Age - years (IQR) & $69(61-74)$ & $66(58-75)$ & 0.550 \\
\hline Weight - kg (IQR) & $82.0(73.0-95.5)$ & $78.6(69.5-94.1)$ & 0.088 \\
\hline Height - cm (IQR) & $174(1.67-1.80)$ & $1.70(1.67-1.76)$ & 0.090 \\
\hline$B S A-m^{2}(I Q R)$ & $2.01(1.83-2.17)$ & $1.93(1.79-2.12)$ & 0.094 \\
\hline \multicolumn{4}{|l|}{ Antiplatelet and -coagulant medication } \\
\hline No medication - no. (\%) & $3(3.1)$ & $3(4.1)$ & 1.000 \\
\hline Antiplatelet medication only - no. (\%) & $75(78.1)$ & $66(90.4)$ & 0.039 \\
\hline Acetylsalicylic acid only - no. (\%) & $65(86.7)$ & $54(81.8)$ & 0.490 \\
\hline Clopidogrel only - no. (\%) & $1(1.3)$ & $0(0.0)$ & 1.000 \\
\hline Dual antiplatelet medication - no. (\%) & $9(12.0)$ & $12(18.2)$ & 0.349 \\
\hline Bridged VKA only - no. (\%) & $10(10.4)$ & $3(4.1)$ & 0.154 \\
\hline Therapeutic LMWH only - no. (\%) & $1(1.0)$ & $0(0.0)$ & 1.000 \\
\hline DOAC only - no. (\%) & $0(0.0)$ & $0(0.0)$ & 1.000 \\
\hline Combined medication use - no. (\%) & $7(7.3)$ & $1(1.4)$ & 0.140 \\
\hline \multicolumn{4}{|l|}{ Clinical and laboratory characteristics } \\
\hline Haematocrit - L/L (IQR) & $0.42(0.39-0.44)$ & $0.41(0.39-0.43)$ & 0.127 \\
\hline Thrombocyte count $-\times 10^{y} / L(I Q R)^{\pi}$ & $228(184-279)$ & $244(205-279)$ & 0.134 \\
\hline INR - s/s (IQR) & $1.02(0.99-1.06)$ & $0.97(0.95-1.01)$ & $<0.001$ \\
\hline Blood group 0/A/B/AB - no. (\%) & $\begin{array}{c}42 / 45 / 7 / 2 \\
(43.8 / 46.9 / 7.3 / 2.1)\end{array}$ & $\begin{array}{c}31 / 37 / 3 / 2 \\
(42.5 / 50.7 / 4.1 / 2.7)\end{array}$ & 0.819 \\
\hline MDRD-eGFR - mL/min/1.73m² (IQR) & $77.9(62.8-92.8)$ & $78.0(61.1-91.5)$ & 0.705 \\
\hline Elective surgery - no. (\%) & $87(90.6)$ & $68(93.2)$ & 0.779 \\
\hline \multicolumn{4}{|l|}{ Type of surgery } \\
\hline Off-pump CABG surgery - no. (\%) & $3(3.1)$ & $4(5.5)$ & 0.467 \\
\hline Logistic EuroScore - (IQR) & $2.85(1.61-5.48)$ & $2.65(1.51-5.28)$ & 0.700 \\
\hline Low mortality risk group - no. (\%) & $30(31.3)$ & $27(37.0)$ & 0.512 \\
\hline Medium mortality risk group - no. (\%) & $45(46.9)$ & $31(42.5)$ & 0.640 \\
\hline High mortality risk group - no. (\%) & $21(21.9)$ & $15(20.5)$ & 0.852 \\
\hline
\end{tabular}

Table S2 Intra- and postoperative variables of the isolated CABG surgery subgroup

\begin{tabular}{lccc}
\hline Intra-operative variables & $\begin{array}{c}\text { Classical-guided } \\
\text { transfusion }(\mathrm{n}=96)\end{array}$ & $\begin{array}{c}\text { ROTEM-guided } \\
\text { transfusion }(\mathrm{n}=73)\end{array}$ & $p$-value \\
\hline Length of operation - min (IQR) & $170(134-205)$ & $200(165-249)$ & $<0.001$ \\
ECC time - min (IQR) & $68(57-85)$ & $81(60-107)$ & 0.025 \\
Cross-clamping time - min (IQR) & $45(36-60)$ & $61(41-72)$ & 0.003 \\
ACT post ECC - \% of baseline (IQR) & $96.5(89.9-105.8)$ & $100.0(93.1-109.6)$ & 0.142 \\
\hline Postoperative variables & & & 0.235 \\
\hline Haematocrit - L/L (IQR) & $0.30(0.27-0.31)$ & $0.28(0.25-0.31)$ & $<0.001$ \\
Platelet count - x10 $/ \mathrm{L}(\mathrm{IQR})$ & $123(102-156)$ & $154(130-184)$ & 0.784 \\
Fibrinogen level - g/L (IQR) & $2.0(1.5-2.3)$ & $1.9(1.6-2.1)$ & 0.414 \\
Temperature on arrival at ICU - ${ }^{\circ} \mathrm{C}(\mathrm{IQR})$ & $36.1(35.7-36.4)$ & $36.0(35.8-36.3)$ & \\
\hline
\end{tabular}

Results are median (IQR) 
Table S3 Primary and secondary endpoints of the isolated CABG surgery subgroup

\begin{tabular}{|c|c|c|c|}
\hline Primary endpoints & $\begin{array}{l}\text { Classical-guided } \\
\text { transfusion }(n=96)\end{array}$ & $\begin{array}{l}\text { ROTEM-guided } \\
\text { transfusion }(n=73)\end{array}$ & $p$-value \\
\hline Estimated blood loss day one - $\mathrm{mL}$ (IQR) & $930(700-1288)$ & $640(463-848)$ & $<0.001$ \\
\hline Intra-operative - mL (IQR) & $200(100-250)$ & $200(100-385)$ & 0.118 \\
\hline Postoperative - $\mathrm{mL}$ (IQR) & $700(523-1088)$ & $370(290-550)$ & $<0.001$ \\
\hline $1^{\text {st }}$ hour - mL (IQR) & $80(40-110)$ & $60(20-105)$ & 0.081 \\
\hline $2^{\text {nd }}$ hour - $m L$ (IQR) & $70(40-110)$ & $60(30-110)$ & 0.220 \\
\hline Cell saver autologous return - $m L$ (IQR) & $419(331-517)$ & $370(259-500)$ & 0.069 \\
\hline \multicolumn{4}{|l|}{ Allogeneic transfusion products ${ }^{*}$} \\
\hline Patients that received RBC - no. (\%) & $46(47.9)$ & $25(26.0)$ & 0.059 \\
\hline On day of operation - units & $0(0-2) ; 1.2[0-9]$ & $0(0-1) ; 0.5[0-6]$ & 0.010 \\
\hline Intra-operative - units & $0(0-0) ; 0.4[0-2]$ & $0(0-0) ; 0.3[0-5]$ & 0.107 \\
\hline Patients that received FFP - no. (\%) & $11(11.5)$ & $1(1.4)$ & 0.014 \\
\hline On day of operation - units & $0(0-0) ; 0.2[0-6]$ & $0(0-0) ; 0.1[0-6]$ & 0.217 \\
\hline Intra-operative - units & $0(0-0) ; 0.0[0-3]$ & $0(0-0) ; 0.1[0-4]$ & 0.739 \\
\hline Patients that received TC - no. (\%) & $17(17.7)$ & $7(9.6)$ & 0.182 \\
\hline On day of operation - units & $0(0-0) ; 0.2[0-2]$ & $0(0-0) ; 0.1[0-2]$ & 0.757 \\
\hline Intra-operative - units & $0(0-0) ; 0.1[0-2]$ & $0(0-0) ; 0.1[0-1]$ & 0.370 \\
\hline \multicolumn{4}{|l|}{ Prohaemostatic medication $^{*}$} \\
\hline Patients that received TXA - no. (\%) & $95(99.0)$ & $73(100)$ & 1.000 \\
\hline On day of operation - $\mathrm{g}$ & $3(3-3) ; 3.2[0-5]$ & $3(3-3) ; 3.0[2-5]$ & 0.215 \\
\hline Intra-operative - $\mathrm{g}$ & 3 (3-3); $2.9[0-5]$ & $3(3-3) ; 3.0[2-5]$ & 0.232 \\
\hline Patients that received PTM - no. (\%) & $96(100)$ & $70(95.9)$ & 0.079 \\
\hline On day of operation - mg & $\begin{array}{c}300(250-300) ; \\
292[50-500]\end{array}$ & $\begin{array}{c}250(250-300) \\
272[0-500]\end{array}$ & 0.057 \\
\hline Intra-operative - mg & $\begin{array}{l}300(250-300) \\
292[50-500]\end{array}$ & $\begin{array}{l}250(250-300) ; \\
272[0-500]\end{array}$ & 0.057 \\
\hline Patients that received DDAVP- no. (\%) & $15(15.6)$ & $0(0.0)$ & $<0.001$ \\
\hline On day of operation - $\mu \mathrm{g}$ & $0(0-0) ; 3.8[0-30]$ & $0(0-0) ; 0.0[0-0]$ & 0.001 \\
\hline Intra-operative - $\mu \mathrm{g}$ & $0(0-0) ; 0.8[0-30]$ & $0(0-0) ; 0.0[0-0]$ & 0.129 \\
\hline Patients that received FC - no. (\%) & $2(2.1)$ & $12(16.4)$ & 0.001 \\
\hline On day of operation - $\mathrm{g}$ & $0(0-0) ; 0.0[0-2]$ & $0(0-0) ; 0.4[0-4]$ & 0.001 \\
\hline Intra-operative - g & $0(0-0) ; 0.0[0-0]$ & $0(0-0) ; 0.3[0-4]$ & $<0.001$ \\
\hline Patients that received rFVIIa - no. (\%) & $0(0.0)$ & $0(0.0)$ & 1.000 \\
\hline On day of operation - mg & $0(0-0) ; 0.0[0-0]$ & $0(0-0) ; 0.0[0-0]$ & 1.000 \\
\hline Intra-operative - mg & $0(0-0) ; 0.0[0-0]$ & $0(0-0) ; 0.0[0-0]$ & 1.000 \\
\hline Patients that received CG - no. (\%) & $15(15.6)$ & $7(9.6)$ & 0.356 \\
\hline On day of operation - mmol & $0(0-0) ; 0.6[0-9.2]$ & $0(0-0) ; 0.1[0-2.3]$ & 0.048 \\
\hline Intra-operative - mmol & $0(0-0) ; 0.3[0-9.2]$ & $0(0-0) ; 0.1[0-2.3]$ & 0.122 \\
\hline \multicolumn{4}{|l|}{ Secondary endpoints ${ }^{*}$} \\
\hline Rethoracotomy - no. (\%) & $11(11.5)$ & $2(2.7)$ & 0.042 \\
\hline Due to bleeding - no. (\%)" & $8(72.7)$ & $1(50)$ & 1.000 \\
\hline ICU length of stay - days & $1(1-2) ; 3.0[0-65]$ & $1(1-1) ; 1.4[0-6]$ & 0.506 \\
\hline Hospital length of stay - days & $8(6-12) ; 12.3[5-81]$ & $8(6-12) ; 9.5[1-30]$ & 0.292 \\
\hline 30-day mortality - no. (\%) & $0(0.0)$ & $2(2.7)$ & 0.185 \\
\hline Lost-to-follow-up - no. (\%) & $5(5.2)$ & $11(15.1)$ & 0.036 \\
\hline
\end{tabular}

"Results are median (IQR); mean [min-max]

" Composite endpoint of rethoracotomy for tamponade, thorax drain bleeding, or mixed reasons 


\section{Chapter 7}

Table S4 Estimation of costs and potential savings due to implementation of a ROTEM-guided algorithm in isolated CABG surgery

\begin{tabular}{lrrr}
\hline Costs - Euro per patient & $\begin{array}{c}\text { Classical-guided } \\
\text { transfusion }\end{array}$ & $\begin{array}{c}\text { ROTEM-guided } \\
\text { transfusion }\end{array}$ & $\begin{array}{r}\text { Potential } \\
\text { savings }\end{array}$ \\
\hline Allogeneic transfusion products - total & & & 391.91 \\
Red blood cell concentrate & 416.79 & 162.09 & 254.70 \\
Fresh frozen plasma & 75.69 & 14.77 & 60.92 \\
$\quad$ Thrombocyte concentrate & 137.32 & 61.03 & 76.29 \\
\hline Prohaemostatic medication - total & & & -112.25 \\
$\quad$ Tranexamic acid & 20.47 & 19.08 & 1.39 \\
Protamine & 10.50 & 9.76 & 0.74 \\
DDAVP & 6.70 & 0.00 & 6.70 \\
Fibrinogen concentrate & 34.88 & 156.01 & -121.13 \\
Activated recombinant factor VII & 0.00 & 0.00 & 0.00 \\
Calcium gluconate & 0.17 & 0.12 & 0.05 \\
\hline Laboratory tests - total & & & -45.50 \\
ROTEM & 28.79 & 87.05 & -58.26 \\
Activated partial thromboplastin time & 9.09 & 4.47 & 4.62 \\
Prothrombin time as INR & 1.88 & 0.63 & 1.25 \\
Fibrinogen level & 12.62 & 10.71 & 1.91 \\
Thrombocyte count & 19.16 & 14.18 & 4.98 \\
\hline Secondary endpoints - total & & & 4309.40 \\
Rethoracotomy for bleeding cause & 655.04 & 107.68 & 547.36 \\
ICU length of stay & 6640.27 & 3241.56 & 3398.71 \\
$\quad$ Non ICU hospital length of stay & 2665.74 & 2302.41 & 363.33 \\
\hline Grand total & & & 4543.56 \\
\hline Based
\end{tabular}

"Based of means of each cohort and the current NZa tariffs

"* Composite endpoint of rethoracotomy for tamponade, thorax drain bleeding, or mixed reasons 
Table S5 Baseline characteristics of the subgroup prone to large consumption of transfusion products (high EuroScore)

\begin{tabular}{|c|c|c|c|}
\hline Baseline characteristics & $\begin{array}{l}\text { Classical-guided } \\
\text { transfusion }(n=76)\end{array}$ & $\begin{array}{l}\text { ROTEM-guided } \\
\text { transfusion }(n=53)\end{array}$ & $p$-value \\
\hline \multicolumn{4}{|l|}{ Demographics } \\
\hline Male gender - no. (\%) & $34(44.7)$ & $30(56.6)$ & 0.213 \\
\hline Age - years (IQR) & 75 (70-79) & $73(66-79)$ & 0.170 \\
\hline Weight - kg (IQR) & $75.0(67.0-87.8)$ & $75.3(63.4-85.5)$ & 0.765 \\
\hline Height - cm (IQR) & $168(163-175)$ & $169(161-175)$ & 0.918 \\
\hline$B S A-m^{2}(I Q R)$ & $1.84(1.74-2.03)$ & $1.86(1.70-2.04)$ & 0.774 \\
\hline \multicolumn{4}{|l|}{ Antiplatelet and -coagulant medication } \\
\hline No medication - no. (\%) & $10(13.2)$ & $9(17.0)$ & 0.617 \\
\hline Antiplatelet medication only - no. (\%) & $40(52.6)$ & $30(56.6)$ & 0.721 \\
\hline Acetylsalicylic acid only - no. (\%) & $26(65.0)$ & $27(90.0)$ & 0.023 \\
\hline Clopidogrel only - no. (\%) & $0(0.0)$ & $0(0.0)$ & 1.000 \\
\hline Dual antiplatelet medication - no. (\%) & $14(35.0)$ & $3(10.0)$ & 0.023 \\
\hline Bridged VKA only - no. (\%) & $18(23.7)$ & $10(18.9)$ & 0.665 \\
\hline Therapeutic LMWH only - no. (\%) & $1(1.3)$ & $0(0.0)$ & 1.000 \\
\hline DOAC only - no. (\%) & $0(0.0)$ & $0(0.0)$ & 1.000 \\
\hline Combined medication use - no. (\%) & $7(9.2)$ & $4(7.5)$ & 1.000 \\
\hline \multicolumn{4}{|l|}{ Clinical and laboratory characteristics } \\
\hline Haematocrit - L/L (IQR) & $0.40(0.37-0.42)$ & $0.39(0.36-0.42)$ & 0.437 \\
\hline Thrombocyte count - $\times 10^{9} / \mathrm{L}(\mathrm{IQR})$ & $238(186-283)$ & $231(200-274)$ & 0.786 \\
\hline INR - s/s (IQR) & $1.04(1.00-1.12)$ & $0.98(0.97-1.10)$ & 0.005 \\
\hline Blood group 0/A/B/AB - no. (\%) & $\begin{array}{c}30 / 43 / 3 / 0 \\
(395 / 56.6 / 39 / 0.0)\end{array}$ & $\begin{array}{c}24 / 25 / 3 / 1 \\
(45.3 / 47.2 / 5.7 / 19)\end{array}$ & 0.492 \\
\hline MDRD-eGFR - $\mathrm{mL} / \mathrm{min} / 1.73 \mathrm{~m}^{2}(\mathrm{IQR})^{\mathrm{n \pi x}}$ & $62.8(54.5-80.0)$ & $60.5(42.8-77.3)$ & 0.217 \\
\hline Elective surgery - no. (\%) & $63(82.9)$ & $48(90.6)$ & 0.303 \\
\hline \multicolumn{4}{|l|}{ Type of surgery } \\
\hline Isolated CABG surgery - no. (\%) & $21(27.6)$ & $15(28.3)$ & 1.000 \\
\hline Off-pump CABG surgery - no. (\%) & $0(0.0)$ & $0(0.0)$ & 1.000 \\
\hline Isolated AVR surgery - no. (\%) & $28(36.8)$ & $15(28.3)$ & 0.347 \\
\hline TAVI procedure - no. $(\%)$ & $20(71.4)$ & $10(66.7)$ & 0.742 \\
\hline Combined CABG/AVR surgery - no. (\%) & $6(7.9)$ & $5(9.4)$ & 0.759 \\
\hline Other procedures - no. (\%) & $21(27.6)$ & $18(34.0)$ & 0.444 \\
\hline Logistic EuroScore - (IQR) & $11.7(7.7-19.1)$ & $11.8(8.1-15.8)$ & 0.743 \\
\hline
\end{tabular}

Table S6 Intra- and postoperative variables of the subgroup prone to large consumption of transfusion products (high EuroScore)

\begin{tabular}{lccc}
\hline Intra-operative variables & $\begin{array}{c}\text { Classical-guided } \\
\text { transfusion }(\mathrm{n}=76)\end{array}$ & $\begin{array}{c}\text { ROTEM-guided } \\
\text { transfusion }(\mathrm{n}=53)\end{array}$ & $p$-value \\
\hline Length of operation - min (IQR) & $165(118-221)$ & $258(171-342)$ & $<0.001$ \\
ECC time - min (IQR) & $89(67-144)$ & $135(91-183)$ & 0.003 \\
Cross-clamping time - min (IQR) & $63(44-93)$ & $85(63-112)$ & 0.010 \\
ACT post ECC - \% of baseline (IQR) & $96.4(86.6-105.5)$ & $102.8(93.4-115.6)$ & 0.013 \\
\hline Postoperative variables & & & \\
\hline Haematocrit $-\mathrm{L} / \mathrm{L}(\mathrm{IQR})$ & $0.29(0.26-0.31)$ & $0.29(0.26-0.33)$ & 0.463 \\
Platelet count $-\mathrm{x} 10^{9} / \mathrm{L}(\mathrm{IQR})$ & $118(94-157)$ & $130(112-159)$ & 0.063 \\
Fibrinogen level $-\mathrm{g} / \mathrm{L}(\mathrm{IQR})$ & $2.2(1.7-2.6)$ & $2.0(1.7-2.4)$ & 0.546 \\
Temperature on arrival at ICU - ${ }^{\circ} \mathrm{C}(\mathrm{IQR})$ & $36.3(25.9-36.6)$ & $36.1(35.8-36.4)$ & 0.104 \\
\hline
\end{tabular}

Results are median (IQR) 
Table S7 Primary and secondary endpoints of the subgroup prone to large consumption of transfusion products (high EuroScore)

\begin{tabular}{|c|c|c|c|}
\hline Primary endpoints & $\begin{array}{l}\text { Classical-guided } \\
\text { transfusion }(n=76)\end{array}$ & $\begin{array}{l}\text { ROTEM-guided } \\
\text { transfusion }(n=53)\end{array}$ & $p$-value \\
\hline Estimated blood loss day one - $\mathrm{mL}$ (IQR) & $785(400-1405)$ & $478(258-815)$ & 0.021 \\
\hline Intra-operative - mL (IQR) & $100(0-265)$ & $200(50-400)$ & 0.022 \\
\hline Postoperative - mL (IQR) & $575(333-1123)$ & $320(185-483)$ & $<0.001$ \\
\hline $1^{\text {st }}$ hour - mL (IQR) & $70(40-110)$ & $50(0-100)$ & 0.053 \\
\hline $2^{\text {nd }}$ hour - $\mathrm{mL}$ (IQR) & $60(30-130)$ & $40(15-105)$ & 0.073 \\
\hline Cell saver autologous return - $\mathrm{mL}$ (IQR) & $502(424-802)$ & $400(250-672)$ & 0.019 \\
\hline \multicolumn{4}{|l|}{ Allogeneic transfusion products ${ }^{*}$} \\
\hline Patients that received RBC - no. (\%) & $49(64.5)$ & $34(64.2)$ & 1.000 \\
\hline On day of operation - units & $1(0-3) ; 2.3[0-18]$ & $0(0-2) ; 1.3[0-8]$ & 0.114 \\
\hline Intra-operative - units & $0(0-2) ; 1.3[0-12]$ & $0(0-1) ; 0.8[0-8]$ & 0.200 \\
\hline Patients that received FFP - no. (\%) & $19(25.0)$ & $9(17.0)$ & 0.386 \\
\hline On day of operation - units & $0(0-0) ; 1.0[0-12]$ & $0(0-0) ; 0.6[0-6]$ & 0.420 \\
\hline Intra-operative - units & $0(0-0) ; 0.6[0-9]$ & $0(0-0) ; 0.5[0-6]$ & 0.527 \\
\hline Patients that received TC - no. (\%) & $26(34.2)$ & $16(30.2)$ & 0.704 \\
\hline On day of operation - units & $0(0-1) ; 0.5[0-4]$ & $0(0-1) ; 0.4[0-3]$ & 0.638 \\
\hline Intra-operative - units & $0(0-1) ; 0.3[0-3]$ & $0(0-1) ; 0.3[0-3]$ & 1.000 \\
\hline \multicolumn{4}{|l|}{ Prohaemostatic medication $^{*}$} \\
\hline Patients that received TXA - no. (\%) & $56(73.7)$ & $52(98.1)$ & $<0.001$ \\
\hline On day of operation - $\mathrm{g}$ & $3(0-3) ; 2.6[0-6]$ & $3(3-3) ; 2.9[1-5]$ & 0.709 \\
\hline Intra-operative - g & $3(0-3) ; 2.2[0-4]$ & $3(3-3) ; 2.8[1-4]$ & 0.024 \\
\hline Patients that received PTM - no. (\%) & $63(82.9)$ & $51(96.2)$ & 0.025 \\
\hline On day of operation - mg & $\begin{array}{c}250(81-300) \\
217 \text { [0-700] }\end{array}$ & $\begin{array}{c}250(200-300) \\
236[0-470]\end{array}$ & 0.581 \\
\hline Intra-operative - mg & $\begin{array}{r}250(81-300) \\
216[0-700]\end{array}$ & $\begin{array}{c}250(200-300) ; \\
236[0-470]\end{array}$ & 0.569 \\
\hline Patients that received DDAVP- no. (\%) & $19(25.0)$ & $1(1.9)$ & $<0.001$ \\
\hline On day of operation - $\mu \mathrm{g}$ & $0(0-11) ; 6.2[0-30]$ & $0(0-0) ; 0.6[0-30]$ & $<0.001$ \\
\hline Intra-operative - $\mu \mathrm{g}$ & $0(0-0) ; 0.8[0-30]$ & $0(0-0) ; 0.0[0-0]$ & 0.236 \\
\hline Patients that received FC - no. (\%) & $7(9.2)$ & $15(28.3)$ & 0.008 \\
\hline On day of operation - $\mathrm{g}$ & $0(0-0) ; 0.3[0-8]$ & $0(0-2) ; 0.7[0-4]$ & 0.005 \\
\hline Intra-operative - g & $0(0-0) ; 0.2[0-8]$ & $0(0-2) ; 0.7[0-4]$ & $<0.001$ \\
\hline Patients that received rFVIla - no. (\%) & $1(1.3)$ & $0(0.0)$ & 1.000 \\
\hline On day of operation - mg & $0(0-0) ; 0.1[0-10]$ & $0(0-0) ; 0.0[0-0]$ & 0.404 \\
\hline Intra-operative - mg & $0(0-0) ; 0.1[0-10]$ & $0(0-0) ; 0.0[0-0]$ & 0.404 \\
\hline Patients that received CG - no. (\%) & $15(19.7)$ & $7(13.2)$ & 0.476 \\
\hline On day of operation - mmol & $0(0-0) ; 1.1[0-20.7]$ & $0(0-0) ; 0.3[0-9.2]$ & 0.091 \\
\hline Intra-operative - mmol & $0(0-0) ; 0.8[0-20.7$ & $0(0-0) ; 0.2[0-6.9]$ & 0.049 \\
\hline \multicolumn{4}{|l|}{ Secondary endpoints ${ }^{*}$} \\
\hline Rethoracotomy - no. (\%) & $15(19.7)$ & $10(18.9)$ & 1.000 \\
\hline Due to bleeding - no. $(\%)^{*}$ & $8(53.3)$ & $7(70.0)$ & 0.679 \\
\hline ICU length of stay - days & $1(1-3)$ & $1(1-3)$ & 0.875 \\
\hline Hospital length of stay - days & $11(8-20)$ & $8(7-14)$ & 0.008 \\
\hline 30-day mortality - no. (\%) & $4(5.3)$ & $6(11.2)$ & 0.316 \\
\hline Lost-to-follow-up - no. (\%) & $3(3.9)$ & $2(3.8)$ & 1.000 \\
\hline
\end{tabular}

"Results are median (IQR); mean [min-max]

* Composite endpoint of rethoracotomy for tamponade, thorax drain bleeding, or mixed reasons 
Table S8 Estimation of costs and potential savings due to implementation of a ROTEM-guided algorithm in the subgroup prone to large consumption of transfusion products (high EuroScore)

\begin{tabular}{lccr}
\hline Costs - Euro per patient & $\begin{array}{c}\text { Classical-guided } \\
\text { transfusion }\end{array}$ & $\begin{array}{c}\text { ROTEM-guided } \\
\text { transfusion }\end{array}$ & $\begin{array}{c}\text { Potential } \\
\text { savings }\end{array}$ \\
\hline Allogeneic transfusion products - total & & & 163.30 \\
Red blood cell concentrate & 764.12 & 690.44 & 73.68 \\
Fresh frozen plasma & 238.13 & 199.37 & 38.76 \\
$\quad$ Thrombocyte concentrate & 356.02 & 305.16 & 50.86 \\
\hline Prohaemostatic medication - total & & & -38.77 \\
$\quad$ Tranexamic acid & 16.82 & 18.59 & -1.77 \\
Protamine & 7.80 & 8.46 & -0.66 \\
DDAVP & 11.01 & 0.95 & 10.06 \\
Fibrinogen concentrate & 148.76 & 300.21 & -151.45 \\
Activated recombinant factor VII & 104.94 & 0.00 & 104.94 \\
Calcium gluconate & 0.32 & 0.21 & 0.11 \\
\hline Laboratory tests - total & & & -103.85 \\
ROTEM & 2.46 & 107.36 & -104.90 \\
Activated partial thromboplastin time & 9.09 & 10.17 & -1.08 \\
Prothrombin time as INR & 5.02 & 3.85 & 1.17 \\
Fibrinogen level & 14.53 & 13.89 & 0.64 \\
Thrombocyte count & 20.18 & 19.86 & 0.32 \\
\hline Secondary endpoints - total & & & 3225.08 \\
Rethoracotomy for bleeding cause & 827.42 & 1038.18 & -210.76 \\
ICU length of stay & 7798.61 & 5181.11 & 2617.50 \\
$\quad$ Non ICU hospital length of stay & 3686.24 & 2867.90 & 818.34 \\
\hline Grand total & & & 3245.76 \\
\hline Based
\end{tabular}

"Based of means of each cohort and the current NZa tariffs

"*omposite endpoint of rethoracotomy for tamponade, thorax drain bleeding, or mixed reasons 



\section{Chapter 8}

\section{General discussion} and summary 



\section{Chapter 8}

\section{General discussion}

In this thesis, I first have outlined the principles of haemostasis. Traditionally haemostasis is divided into a primary and secondary haemostasis part, both leading to fibrin formation; subsequent fibrin breakdown is regulated by fibrinolysis. The interplay of coagulation enzymes, blood platelets, and the vessel wall, is however more elaborate than what has been delineated in chapter two. ${ }^{1,2}$ Instead of separate processes occurring in parallel, coagulation and fibrinolysis evolve at the same time. While the clot starts to grow due to the increased amount of activated platelets trapped in the expanding fibrin mesh, fibrinolytic enzymes start to shape and break down the growing clot right away. Moreover, a more 'modern' view on coagulation also adopts the influence of inflammatory processes on coagulation. ${ }^{1,3}$

However, it is sometimes more practical to consider distinct elements of the coagulation process, as most laboratory tests focus on a limited part of coagulation. ${ }^{1}$ Platelet function testing is usually performed in isolated platelet rich plasma (PRP), thereby excluding the effect of the vessel wall and that of other blood constituents. Light transmission aggregometry (LTA) as the gold standard in platelet function testing in PRP might be replaced in the future by whole blood platelet function testing methods which adopt a more holistic view on haemostasis. Whole blood tests already have proven that they have added value compared to LTA, because of practical reasons including reduced time consumption and the ease of analysis and interpretation. Multiple electrode aggregometry (MEA or Multiplate) and the platelet function analyzer 100 (PFA-100) both use whole blood and thus, in theory, the effects of red blood cells are evaluated in these tests as well. One of the newest methods of haemostasis monitoring with focus on the platelet component are flow dependent microscopy analysis, such as pioneered in the laboratory of Prof. Heemskerk at the Maastricht University. A paper describing the protocol involved in these analyses has set the standard in these flow chamber experiments. ${ }^{4}$ This technology incorporates whole blood, 


\section{Chapter 8}

flow, and can even mimic vessel wall elements. By varying different components in these chambers (low flow vs. high flow; different coating types; etc.), haemostasis under different conditions can by evaluated. A disadvantage of this technique remains the point-of-care aspect, because a well-equipped professional laboratory is needed. PFA-100, in contrast to MEA, also measures platelet function under flow conditions. This has made the PFA-100 an excellent screening tool for von Willebrand disease, as the multimeres unfold more or unfold less depending of the flow rate.

Viscoelastic whole blood tests, like rotational thromboelastometry (ROTEM) or thrombelastography (TEG), evaluate clot formation and breakdown as an overall process of haemostasis, similar to the cell-based concept of coagulation in vivo. ${ }^{1}$ In contrast to pure plasma based tests, like the prothrombin time (PT) or activated partial thromboplastin time (aPTT), haemostasis is tested in a more holistic manner. During the analysis, clot formation and breakdown, facilitated by platelets together with the plasmatic and fibrinolytic enzymes, are visualized in real-time using graphs and numbers. This can be done bedside without involvement of specialized laboratory personnel using the latest addition to the rotational thromboelastometry portfolio of the ROTEM sigma or by using the TEG $6 S$ from Haemonetics. On the other hand, the PT and aPTT, as the cornerstones of conventional plasma based coagulation tests, remain valuable as they specifically address medication effects of vitamin $\mathrm{K}$ antagonist and unfractionated heparin. Both PT and aPTT are still used as screening tests in suspected bleeding disorders in daily practice. For instance, a prolonged aPTT could have multiple causes (table 2.1) and by evaluating separate coagulation factors, an isolated deficiency in coagulation factor VIII leads to the diagnosis of haemophilia A. In contrast, standard viscoleastic whole blood point-of-care methods are insensitive for disorders such as haemophilia A. However, after tweaking of the protocols by varying TF amounts or by adding tissue type plasminogen activator (TPA), clot resistance in haemophilia A patients treated with recombinant activated FVII could be shown. ${ }^{5,6}$ In acquired multifactorial disorders of haemostasis the use of viscoelastic tests is better applicable; in traumatic bleeding disorders ROTEM assesses both clot formation, clot stability, and 
clot breakdown in one test cycle. Because multiple channels can be used at the same time, the effects of fibrinogen, heparin, and the contribution of an antifibrinolytic agent in these tests can be evaluated separately and in parallel. With the ROTEM sigma, all-in-one cartridges are employed to reduce the complexity of starting a measurement. The global haemostasis cartridge used in the TEG 6S has similar capabilities to the ROTEM sigma, but is different in the details.

Thrombin as the central controlling enzyme is involved in clot formation as well as in clot breakdown. Basically, too much thrombin could lead to a prothrombotic state, while too little thrombin is seen in bleeding disorders. Another innovation in the field of coagulation monitoring enables measuring the amount of thrombin generated using the calibrated automated thrombogram. ${ }^{7}$ Essentially, the amount of thrombin formed is measured using a fluorogenic substrate based analysis. The thrombin generation test used to be a plasma based analysis, but it has evolved towards a point-of-care whole blood device, quite recently. $^{7}$

Similarly to the shift in the use of conventional plasma based tests to whole blood based point-of-care tests, a shift in transfusion practice takes place, from the use of whole blood products, to fractionated blood components, and further to purified, isolated coagulation proteins. Especially in traumatic bleeding, the replacement of whole blood by fractioned transfusion products, like red blood cell concentrates, fresh frozen plasma or cryoprecipitate, and platelet concentrates is noticeable. More recently, purified coagulations protein like fibrinogen concentrate or recombinant activated FVII and the use of tranexamic acid have gained much interest as a more goal-directed therapeutic approach in acute bleeding disorders. ${ }^{8}$

Pitfalls of whole blood point-of-care methods of haemostasis testing are that, although its use is simple, validation of the method and quality insurance over time needs to be established. Deviations of results due to, for instance, inadequate filling of blood collection tubes, improper use and wear-and-tear of pointof-care devices need to be addressed when being used in daily clinical care. Physicians and other non-laboratory medical personnel can be educated to perform tests on the spot, but in the end they are not sufficiently qualified to 


\section{Chapter 8}

address these matters. Besides, their focus should be on taking care of patients, not on laboratory equipment. For all laboratory tests, pre-analytical variables need to be controlled as much as possible to ensure quality control. ${ }^{9} \mathrm{Hae}-$ molysis will disturb optical plasmatic coagulation assays, low platelet counts and anaemic conditions will affect whole blood platelet function tests like PFA100 and Multiplate, as we showed in chapter three. By correcting the results for these pre-analytical differences among healthy volunteers we were able to define dynamic reference intervals. The effect of red blood cells seemed to be important in the PFA-100 only and not in the Multiplate, based on the different methods used in both devices. The PFA-100 acts by adding flow to the measuring system and thus red blood cells improve platelet adhesion by pushing them towards the outer layer of the bloodstream. ${ }^{10}$ I stated in my introduction chapter: 'for effective primary haemostasis, a sufficient number of functional platelets needs to be present in the body'. In analogy, a sufficient number of platelets needs to be present to measure their function. The fact that the results of the Multiplate device needs to be adjusted for the individual platelet count has been described earlier, however, we could show for the first time how to interpretate whole blood point-of-care platelet function results in patients in need for a biopsy of a previously transplanted kidney. This study presented in chapter four, revealed that in chronic kidney disease (CKD) patients prone for developing bleeding events, because of thrombocytopenia (present in 6\%), anaemia (63\%), frequent use of the antiplatelet medication acetylsalicylic acid $(17 \%)$, and uraemia $(73 \%)$, potential bleeders could not be identified. None of these risk factors, potentially contributing to in vivo platelet dysfunction, emerged as in vitro predictors of bleeding complications. In conclusion, (prior) use of acetylsalicylic acid seemed to be the only predictor of a bleeding event after biopsy of a transplanted kidney. We expected that either PFA-100 or MEA or both would help identify patients prone to bleeding disorders, but as can be observed from figure 4.1 and 4.2, patients with a positive bleeding endpoint were distributed all over the charts. It did not matter if results were or were not adjusted for thrombocytopenia or anaemia. Most strikingly, we observed an in vitro platelet dysfunction in almost half of the patients when using MEA (table 4.3). One could argue that 
von Willebrand factor (vWF) might play a pivotal regulatory role in CKD. WWF levels are increased when age advances, just as the incidence of CKD increases when growing older. On the other hand, uraemia seen in CKD can result in vWF dysfunction. ${ }^{11}$ This notion and the observation that lvy bleeding times (IBT) were prolonged in von Willebrand disease (vWD, especially in type III vWD) made that desmopressin used to be given to patients with prolonged IBT awaiting a kidney biopsy. A direct causal effect of uraemia in CKD on bleeding events is nowadays put in doubt and anaemia seems to be the main culprit. ${ }^{11}$ Although specific haemostatic defects are found in uraemia, growing evidence suggests that in several chronic conditions affecting haemostasis (e.g. in CKD or in cirrhotic liver disease) a reset of the haemostatic balance (homeostasis) is occurring. ${ }^{11,12}$ Because of the abundance of regulatory coagulation proteins, homeostasis of coagulation can be controlled more precisely and effectively, unlike by the means of a simple on/off-switch. This renewed balance seen in cirrhosis and uraemia is unstable and could tip easily to either side, resulting in thrombosis or bleeding. ${ }^{11,12}$ As such, the term 'uraemic thrombocytopathy' seems to be more of an in vitro laboratory finding, than a clinical sign of bleeding due to platelet dysfunction. This conclusion is supported by the fact that we were unable to find a link between elevated or high blood urea concentrations and increased bleeding endpoints (non-significant odds ratios) in our study.

Bleeding is mostly multifactorial, but slight (or large) disorders in a specific part of the haemostatic system can result in major bleeding complications even after minor trauma. The pathogenesis of traumatic bleeding is an interesting phenomenon. ${ }^{13,14}$ Endothelial damage initiates the formation of the tenase complex by expressing tissue factor (TF) to the blood on TF bearing cells after which thrombin is produced in abundance while further being amplified through the Josso loop. Thrombin with its central controlling role in haemostasis is halted when in complex with endothelial thrombomodulin. Next, protein $\mathrm{C}$ will be activated by the thrombin-thrombomodulin complex and fibrin formation by the diminishing thrombin will be further attenuated. Activated protein $\mathrm{C}$ also negates PAI-1; PAI-1 is then unable to block the by endothelial damage released tPA. The end result is anticoagulation and hyperfibrinolysis in which hyperfibrinolysis 


\section{Chapter 8}

is predominant. ${ }^{15}$ One can argue that homeostasis will find a renewed balance in haemostasis, but in traumatic bleeding this sophisticated system seems to fail. ${ }^{14}$ The overt bleeding, tissue hypoxemia due to shock, shedding of the glycocalyx, subsequent acidosis, inflammatory response, and need for fluid resuscitation all contribute to a lethal triad of dilutional consumptive coagulopathy by trauma: acute coagulopathy of traumatic shock (ACoTS). ${ }^{14}$ Viscoelastic methods are considered the best tools for fast and adequate detection of hyperfibrinolysis in trauma, ${ }^{16,17}$ however mild to moderate fibrinolysis can easily be missed even by viscoelastic tests. ${ }^{18}$ Our research of the validated tPA induced fibrinolysis assay for ROTEM was not performed in ACoTS, because of lack of steady inclusion rates of trauma patients at that time. On the other hand, the antifibrinolytic effects of the tranexamic acid could be demonstrated effectively in the group of post cardiac surgery patients using our method (chapter five). The euglobulin clot lysis time (ECLT) as the plasma based gold standard assay for fibrinolysis detection, was insensitive for tranexamic acid therapy. On the other hand, good agreement between our method and the ECLT on hypofibrinolysis detection in sepsis was apparent. The high antifibrinolytic effect of PAI-1 together with the prothrombotic potential due to high fibrinogen levels were deemed to be responsible for the results in both tests. From a scientific point of view likely, the TPA induced fibrinolysis protocol for ROTEM should be tested in ACoTS, but after the CRASH -2 trial and subsequent analyses, ${ }^{19}$ most, if not all, trauma patients will be treated with tranexamic acid before haemostasis monitoring can be initiated. Blood sampling before tranexamic acid treatment could prove the hypothesis that our method is able to distinguish fast and reliable between trauma patients if hyperfibrinolysis is not, moderately, or overtly present. Thereby, it would be possible to guide tranexamic acid therapy and administer it to those who would benefit from it and refrain clinicians from using a regime of 'tranexamic acid for all'. ${ }^{14}$ To me this seems to be an interesting investigational study. Of note, a similar non-validated method has been performed in a study using the TEG and thus this concept seems to be promising for the validated ROTEM method as well. ${ }^{20}$ Another interesting opportunity of research, could be to address the systemic effects of intravenous thrombolytic therapy for 
massive pulmonary or venous thromboembolism or of intra-arterial treatment for acute ischemic stroke. A PubMed search revealed 31 hits when searching for "(thrombolysis viscoelastic) OR (thrombolysis TEG) OR (thrombolysis ROTEM)", of which only two looked at systemic effects of intravenous thrombolysis therapy in thrombotic disorders. ${ }^{21,22}$ Systemic effects of thrombolytic therapy on lysis parameters could not be demonstrated using standard $\mathrm{TEG}^{21}$ or ROTEM $^{22}$ analysis in these studies.

As time is of the essence in bleeding scenarios and early diagnosis and treatment saves lives, ${ }^{17,18}$ we have also looked at early prediction models of thrombocytopenia and hypofibrinogenemia in cardiac surgery patients for which ROTEM was introduced as standard care in the Maastricht UMC+ (chapter six). We envisioned that ROTEM would replace conventional plasma based coagulation assays for multifactorial bleeding diagnosis. Prerequisites were that ROTEM would do this faster and give more information than conventional laboratory tests, and all of this with preferably only one tube of (citrate anticoagulated) blood. Treatment protocols such as those used in chapter seven normally recommend using amplitude at ten minutes (A10) on EXTEM and FIBTEM to measure overall clot strength and the contribution of fibrinogen. Platelets and fibrinogen, being the most crucial end products in a formed clot, could be deduced from the ROTEM graphs and numbers. FIBTEM as a measurement of clot strength invoked by fibrinogen had a strong positive correlation with fibrinogen levels analysed by the Clauss method. Using the amplitude at five minutes (FIBTEM A5; Pearson's $r$ of 0.87 in respect to Clauss' fibrinogen levels) meant five minutes of time gain over using $A 10$. By subtraction of the amplitude of the FIBTEM from the EXTEM, we could calculate the platelet contribution to the clot strength. The newly introduced PLTEM for estimating platelet counts correlated strongly with conventionally measured platelet counts (PLTEM A5; $r=0.85$ ). The PLTEM amplitude at five minutes correlated even better with platelet counts than amplitudes at ten minutes or when maximum clot strength was reached (MCF). In a letter to the editor, ${ }^{23}$ we re-assessed this PLTEM parameter after a publication which stated that clot elasticity (CE) should be used for evaluating platelet contribution to the formed clot. In hindsight, our more 


\section{Chapter 8}

straightforward approach seemed to be more simple and superior in estimating platelet counts. ${ }^{23}$ It is now possible to use one tube of citrate anticoagulated whole blood to get reliable insights pertaining fibrinogen level, platelet count, overall clot strength, and available fibrinolysis in cardiac surgery patients within 15 minutes of arrival of the sample at the laboratory. Conventional laboratory tests for PT, aPTT, platelet counts and Clauss' determination of fibrinogen require more blood and about 25 minutes longer to give full results, while not giving any information on fibrinolysis parameters.

Implementation of a ROTEM-based treatment protocol cannot only give faster results, but at the same time it can save costs. In my seventh chapter we sought to investigate the impact of introducing ROTEM-based treatment strategies in cardiac surgery patients. Using two cohorts, one in which conventional laboratory tests were used and another in which ROTEM-based decisions were made to monitor and guide haemostasis in cardiac surgery patients, it was possible to show reductions in the use of allogeneic blood products, reduction of hospital length of stay, and cut down in overall costs. Mortality rates were not reduced upon the implementation of a ROTEM-guided transfusion algorithm, likewise, benefit on mortality could not be demonstrated in a recent systematic review and meta-analysis of seven randomized controlled trials. ${ }^{24}$ Relative risk was in favour of ROTEM guidance at 0.55 , but this was not significant with a $95 \%$ confidence interval of $0.28-1.10 .^{24}$ Subgroup analysis of coronary artery bypass graft $(C A B G)$ surgery and of high risk cardiac surgery based on EuroScore, showed also overall costs reduction by using ROTEM-guided transfusion protocols. Both CABG surgery and high EuroScore were chosen in order to reflect homogenous subgroups of cardiac surgery patients. In the isolated CABG surgery subgroup the amount of blood loss and the number of rethoracotomies were reduced at the expense of using more fibrinogen. In the high EuroScore subgroup, blood loss and hospital length of stay were reduced as the two most remarkable findings.

Point-of-care viscoelastic monitoring of haemostasis and platelet function testing seems to be the way forward, as new modalities and research opportunities are introduced regularly, further fine-tuning accuracy, precision, and efficacy of 
these tests. Point-of-care whole blood thrombin generation seems to be the next step in assessing the intricacies of secondary haemostasis (and more), while flow chamber testing will boost research at the primary haemostasis side (and more) to a point-of-care level which ROTEM made us become familiar with. Monitoring of fibrinolysis and the anticoagulation pathways of haemostasis has still a long way to go in the point-of-care era, but our rTPA induced ROTEM fibrinolysis assay may have set this first step forward providing a robust pointof-care concept for the future.

\section{Summary in English}

From chapter two we have learned that haemostasis monitoring can be done in plasma based or whole blood setups. Every single contributing element to haemostasis can be assessed in order to pinpoint a diagnosis. Point-of-care testing differs from conventional laboratory testing in that it has the premisses of enabling bedside application in an easy and fast manner. Platelet function testing is based on the ability to use platelet agonists to induce platelet aggregation. Different assays exist each with their own advantages and disadvantages. The overall ruling seems to be that in order to adequately test platelet function, sufficient amounts of platelets needs to be present in the measuring sample. Correcting for platelet counts, like we did in chapter three, seems to overcome this problem. That bleeding complications are mostly multifactorial and therefore highly unpredictable, is apparent from our study in chapter four. A thorough investigation using point-of-care platelet function testing in transplanted kidney biopsies could show that, although half of the patients seem to have an in vitro platelet function defect, almost none of the patients experienced bleeding complications. In chapter five, our validated method of whole blood viscoelastic testing of the fibrinolytical pathway was proven to be an innovative addition to the conventional plasma based fibrinolysis assays. We could show that the diagnosis of a hypofibrinolytical state could be made quickly in septic patients, while this was not possible by using regular viscoelastic tests. The use of a fibrinolysis blocker (tranexamic acid) in cardiac surgery patients was evidently 


\section{Chapter 8}

picked up by our method in contrast to the conventional plasma based euglobulin clot lysis time assay. The holistic cell based concept in haemostasis testing has proven that whole blood viscoelastic point-of-care assays, like rotational thromboelastometry (ROTEM) can adequately and efficiently diagnose haemostatic disturbances in bleeding scenarios. That time can be saved by using ROTEM is even more evident from our studies in chapter six. Twenty-five minutes could be saved in diagnostic speed over conventional laboratory tests by using the results after just five minutes with ROTEM. Using implemented ROTEM-guided transfusion protocols we were able to reduce hospital length of stay after cardiac surgery and have potentially reduced total costs, as was shown in chapter seven. In conclusion, with point-of-care devices in haemostasis monitoring it is feasible to accurately and quickly pinpoint disorders in platelet function, coagulation, and fibrinolysis. The clinical impact of these findings is however uncertain, but eventually, it might be possible to save patients' lives and reduce healthcare costs.

\section{Summary in Dutch}

In hoofdstuk twee hebben we gezien dat het controleren van de bloedstolling kan met zowel op bloedplasma als op volbloed gebaseerde technieken. Elk los element dat bijdraagt aan de stolling kan in kaart worden gebracht om tot een diagnose te komen met deze technieken. Point-of-care testen verschillen van de conventionele laboratorium testen in het feit dat ze aan het bed van de patient gebruikt kunnen worden op een gemakkelijke en snelle wijze. De basis om bloedplaatjes functie te meten is dat stolsels geïnduceerd worden met bloedplaatjesactivatoren. Er zijn verscheidene testen, elk met hun eigen voor- en nadelen. In het algemeen geldt dat er een voldoende aantal bloedplaatjes nodig is om hun functie nauwkeurig te kunnen meten. Door te corrigeren voor het bloedplaatjesaantal conform het onderzoek in hoofdstuk drie, lukte het ons dit probleem te omzeilen. Dat bloedingscomplicaties meestal meerdere oorzaken hebben en daarom ook moeilijk voorspelbaar zijn, is gebleken uit het onderzoek in hoofdstuk vier. Ondanks grondig onderzoek met point-of-care bloedplaatjesfunctietesten voorafgaand aan nierbiopten van patiënten na een niertransplan- 
tatie, was het niet mogelijk, ondanks dat bijna de helft van hen een in vitro bloedplaatjes functiedefect leek te hebben, een voorspelling te doen over bloedingscomplicaties. In hoofdstuk vijf hebben we laten zien dat onze gevalideerde volbloed viscoelasticiteits methode om fibrinolyse metingen te verrichten een innovatieve aanvulling is op de reguliere plasma gebaseerde fibrinolyse methodes. Een verlaagde fibrinolyse capaciteit in patiënten met bloedvergiftiging (sepsis) kon makkelijk en snel worden opgespoord, terwijl dit niet mogelijk was met de gebruikelijke viscoelastische methodes. Het gebruik van een fibrinolyse remmer (tranexaminezuur) in een patiëntenpopulatie na hartchirurgie kon duidelijk teruggevonden worden met onze methode in tegenstelling tot de conventionele in plasma uitgevoerde euglobuline clotlysis tijd. De holistische benadering van het meten van bloedstolling heeft reeds bewezen dat volbloed point-ofcare methodes zoals ROTEM (rotational tromboelastometry) nauwkeurig en efficiënt stollingsafwijkingen in bloedingssituaties kunnen aantonen. Dat er tijdswinst valt te behalen door ROTEM in te zetten werd duidelijk uit de studies die in hoofdstuk zes zijn beschreven. Het is mogelijk 25 minuten eerder een diagnose te stellen met ROTEM dan dat dit met de gebruikelijke metingen kon. Hiervoor gebruikten we de ROTEM resultaten na vijf minuten. Verder was het mogelijk om vernieuwde op ROTEM gebaseerde transfusieprotocollen in de klinische praktijk in te voeren. In hoofdstuk zeven konden we aantonen dat de ligduur in het ziekenhuis van de patiënten na hartchirurgie verminderd konden worden alsmede de hiermee gemoeide kosten. Concluderend, met point-of-care methodes om de bloedstolling te vervolgen is het mogelijk om nauwkeurig en snel afwijkingen in de bloedplaatjes functie, de stollingscascade en het fibrinolytische systeem aan te duiden. De exacte klinische relevantie van al deze afwijkingen in stollingswaarden is nog onduidelijk, maar mogelijk kan met deze methodes toekomstige patiëntenlevens en ziekenhuiskosten bespaard worden. 


\section{Chapter 8}

\section{References}

1. Ratnoff OD. Some relationships among hemostasis, fibrinolytic phenomena, immunity, and the inflammatory response. Adv Immunol. 1969;10:145-227.

2. Smith SA. The cell-based model of coagulation. J Vet Emerg Crit Care. 2009 Feb;19(1):3-10.

3. Borissoff $\mathrm{JI}$, Spronk HM, ten Cate $\mathrm{H}$. The hemostatic system as a modulator of atherosclerosis. N Engl J Med. 2011 May 5;364(18):1746-60.

4. Van Kruchten R, Cosemans JM, Heemskerk JW. Measurement of whole blood thrombus formation using parallel-plate flow chambers - a practical guide. Platelets. 2012;23(3):229-42.

5. Dargaud Y, Prevost C, Lienhart A, Claude Bordet J, Negrier C. Evaluation of the overall haemostatic effect of recombinant factor VIla by measuring thrombin generation and stability of fibrin clots. Haemophilia. 2011 Nov;17(6):957-61.

6. Young G, Sørensen B, Dargaud Y, Negrier C, Brummel-Ziedins K, Key NS. Thrombin generation and whole blood viscoelastic assays in the management of hemophilia: current state of art and future perspectives. Blood. 2013 Mar 14;121(11):1944-50.

7. Ninivaggi M, Apitz-Castro R, Dargaud Y, de Laat B, Hemker HC, Lindhout T. Whole-blood thrombin generation monitored with a calibrated automated thrombogram-based assay. Clin Chem. 2012 Aug;58(8):1252-9.

8. Stein P, Kaserer A, Sprengel K, Wanner GA, Seifert B, Theusinger OM, Spahn DR. Change of transfusion and treatment paradigm in major trauma patients. Anaesthesia. 2017 May 23.

9. Lippi G, Salvagno GL, Montagnana M, Lima-Oliveira G, Guidi GC, Favaloro EJ. Quality standards for sample collection in coagulation testing. Semin Thromb Hemost. 2012 Sep;38(6):56575.

10. Perkkiö J, Wurzinger LJ, et al. Fåhraeus-Vejlens effect: margination of platelets and leukocytes in blood flow through branches. Thromb Res. 1988 May 1;50(3):357-64.

11. Mannucci PM, Tripodi A. Hemostatic defects in liver and renal dysfunction. Hematology Am Soc Hematol Educ Program. 2012;2012:168-73.

12. Escolar G, Díaz-Ricart M, Cases A. Uremic platelet dysfunction: past and present. Curr Hematol Rep. 2005 Sep;4(5):359-67.

13. Brohi K, Cohen MJ, Davenport RA. Acute coagulopathy of trauma: mechanism, identification and effect. Curr Opin Crit Care. 2007 Dec;13(6):680-5.

14. Walsh M, Shreve J, Thomas S, Moore E, Moore H, Hake D, Pohlman T, Davis P, Ploplis V, Piscoya A, Wegner J, Bryant J, Crepinsek A, Lantry J, Sheppard F, Castellino F. Fibrinolysis in Trauma: "Myth," "Reality," or "Something in Between". Semin Thromb Hemost. 2017 Mar;43(2):200-212.

15. Davenport RA, Guerreiro M, Frith D, Rourke C, Platton S, Cohen M, Pearse R, Thiemermann C, Brohi K. Activated Protein C Drives the Hyperfibrinolysis of Acute Traumatic Coagulopathy. Anesthesiology. 2017 Jan;126(1):115-127. 
16. Hunt H, Stanworth S, Curry N, Woolley T, Cooper C, Ukoumunne O, Zhelev Z, Hyde C. Thromboelastography (TEG) and rotational thromboelastometry (ROTEM) for trauma induced coagulopathy in adult trauma patients with bleeding. Cochrane Database Syst Rev. 2015 Feb 16;(2):CD010438.

17. Rossaint R, Bouillon B, Cerny V, Coats TJ, Duranteau J, Fernández-Mondéjar E, Filipescu D, Hunt BJ, Komadina R, Nardi G, Neugebauer EA, Ozier Y, Riddez L, Schultz A, Vincent JL, Spahn DR. The European guideline on management of major bleeding and coagulopathy following trauma: fourth edition. Crit Care. 2016 Apr 12;20:100.

18. Raza I, Davenport R, Rourke C, Platton S, Manson J, Spoors C, Khan S, De'Ath HD, Allard S, Hart DP, Pasi KJ, Hunt BJ, Stanworth S, MacCallum PK, Brohi K. The incidence and magnitude of fibrinolytic activation in trauma patients. J Thromb Haemost. 2013 Feb;11(2):307-14.

19. Roberts I, Shakur H, Afolabi A, Brohi K, Coats T, Dewan Y, Gando S, Guyatt G, Hunt BJ, Morales $\mathrm{C}$, Perel $\mathrm{P}$, Prieto-Merino $\mathrm{D}$, Woolley $\mathrm{T}$. The importance of early treatment with tranexamic acid in bleeding trauma patients: an exploratory analysis of the CRASH-2 randomised controlled trial. Lancet. 2011 Mar 26;377(9771):1096-101, 1101.e1-2.

20. Chapman MP, Moore EE, Moore HB, Gonzalez E, Gamboni F, Chandler JG, Mitra S, Ghasabyan A, Chin TL, Sauaia A, Banerjee A, Silliman CC. Overwhelming tPA release, not PAl-1 degradation, is responsible for hyperfibrinolysis in severely injured trauma patients. J Trauma Acute Care Surg. 2016 Jan;80(1):16-23; discussion 23-5.

21. McDonald MM, Wetzel J, Fraser S, Elliott A, Bowry R, Kawano-Castillo JF, Cai C, Sangha N, Messier J, Hassler A, Archeval-Lao J, Parker SA, Rahbar MH, Pivalizza EG, Chang TR, Grotta JC. Thrombelastography does not predict clinical response to rtPA for acute ischemic stroke. J Thromb Thrombolysis. 2016 Apr;41(3):505-10.

22. Stanford SN, Sabra A, Lawrence M, Morris RH, Storton S, Wani M, Hawkins K, Williams PR, Potter JF, Evans PA. Prospective evaluation of blood coagulability and effect of treatment in patients with stroke using rotational thromboelastometry. J Stroke Cerebrovasc Dis. 2015 Feb;24(2):304-11.

23. Kuiper GJ, Henskens YM. Rapid and Correct Prediction of Thrombocytopenia and Hypofibrinogenemia with Rotational Thromboelastometry in Cardiac Surgery Reconsidered. J Cardiothorac Vasc Anesth. 2016 Dec;30(6):e55-e56.

24. Serraino GF, Murphy GJ. Routine use of viscoelastic blood tests for diagnosis and treatment of coagulopathic bleeding in cardiac surgery: updated systematic review and meta-analysis. $\mathrm{Br} \mathrm{J}$ Anaesth. 2017 Jun 1;118(6):823-833. 



\section{Chapter 9}

\section{Valorisation}





\section{Chapter 9}

\section{Valorisation}

The roots of this dissertation originate from one big study in which patients' coagulation state was researched using an extensive interview and panel of laboratory tests, the PANE-study. Soon it was known that the intended laboratory tests had major pitfalls and tweaking of these tests was needed to adequately investigate the haemostatic system. One of the goals of the PANE-study was to simplify the pre-operative coagulation screening process by ruling out those patients who do not have an increased bleeding risk during the operation due to the surgery itself or because of the bleeding diathesis of the patients. In October 2011 the Dutch Heart Foundation gave a course on vascular biology in Papendal, the Netherlands. It was at this course that I was awarded the second prize for the 'Workshop Popular Science Communication'. What follows is the Dutch text that I wrote:

\section{Bloedstollend, maar met een gerust hart, de operatiekamer in}

Een operatie ondergaan is voor patiënten een spannende gebeurtenis. Ze worden overgelaten aan de chirurg en 'de man met de hamer', in vakjargon de anesthesioloog. Er wordt geprikt, gesneden, gezaagd en gehecht: bloed! Bloedverlies rondom een operatie wordt zo minimaal mogelijk gehouden door goed samenspel tussen chirurg en anesthesioloog. Maar eigenlijk begint het bloedstelpen al op het spreekuur van de anesthesioloog.

Met een onderzoek onder meer dan 700 patiënten wordt gekeken wat de beste en meest snelle strategie is om patiënten het groene licht te geven voor een operatie. Dit onderzoek in het Maastricht Universitair Medisch Centrum is opgezet vanuit zowel het Hematologisch Laboratorium als de afdeling Anesthesiologie \& Pijnbestrijding. Door naar een juiste combinatie te zoeken tussen allerlei vragen over bloedingsproblemen en bloedtesten in het laboratorium, proberen ze een antwoord te krijgen op de vraag of iemand veilig een operatie kan ondergaan.

Patiëntenveiligheid staat voorop bij een operatie. Een operatie wordt dan ook wel vergeleken met de luchtvaart: alles moet tiptop in orde en gecontroleerd zijn. Zo wordt tijdens een operatie bij elke stap een checklijst afgevinkt om bijvoorbeeld verwisseling te voorkomen. Net zoals de kerosine in het vliegtuig, zo moet ook het bloed van de patiënt van goede kwaliteit zijn voordat de operatie begint.

De bloedstolling wordt uiteraard al langer gemeten in het laboratorium, dat is niets nieuws. Wat het nu wezenlijk anders maakt, is dat de technologie steeds beter wordt. Eerder moest lang gewacht worden op de uitslagen van bloedtesten, maar met de apparatuur die bij dit onderzoek worden ingezet kan met één test veel meer informatie verzameld worden in minder tijd. Verder zijn de apparaten veel compacter zodat ze zelfs aan het bed van de patiënt kunnen worden ingezet.

De onderzoekers durven zelfs te stellen dat in de toekomst de technologie nog geavanceerder wordt, zodat slechts met enkele druppels bloed de gehele bloedstolling in kaart kan worden gebracht. Mocht er een aanwijzing zijn uit de vragen dat er een bloe- 


\section{Chapter 9}

dingsprobleem speelt bij de patiënt, dan zal een simpele vingerprik in korte tijd hier uitsluitsel over geven. Maar dit is nog toekomstmuziek. Voorlopig zal er nog veel onderzoek moeten gebeuren, zodat ook $u$ bloedstollend, maar met een gerust hart, de operatiekamer in en weer uit gaat.

Next is a short English summary of the above Dutch text:

\section{Going for surgery in a blood curdling but relieved way}

An operation can be frightening for patients. Blood loss is encountered and has to be minimized by the surgeon and the anaesthesiologist. The reduction of blood loss, however, is already initiated on the pre-operative screening clinics of the anaesthesiologist.

In the Maastricht University Medical Centre a study has started to examine the best strategy to give patients green light for going for surgery because of a possible bleeding tendency. Using an extensive interview and panel of laboratory tests, safety for surgery is reviewed.

Protocols of the surgical process are commonly compared to aviation. Checklist are used to assess safety during the operation. Like kerosene in aviation, patients' blood needs to be of good quality.

Technology is ever evolving. The laboratory tests in this study are getting smaller, faster, more precise, and can give the same, or similar results, of multiple tests in one go.

Stated is that in the future this technology is getting even more advanced. A single drop of blood could evaluate the full haemostatic system of a patient in little time.

However, this seems to be science fiction, as for now lots of research has to be performed before patients can proceed blood curdling, but relieved, for surgery.

This short assay was envisioning the essence of this thesis back then in 2011 and now emphasizes the bigger picture of this valorisation chapter.

So how can the acquired knowledge from this thesis be of any use?

The gathered knowledge from this dissertation is of great value for patients, hospitals, medical healthcare personnel (e.g. surgeons, anaesthesiologists, perfusionists, blood bankers, et cetera), blood donors, and healthcare technology companies (of ROTEM, MultiPlate, PFA-100, TEG, et cetera) alike.

Although the monitoring principles date back to the 1940's for the viscoelastic measuring devices, the amount of clinical research in haemostasis monitoring has exploded after the rebirth of ROTEM and TEG in the mid-eighties. One of the first publications of viscoelastic monitoring of haemostasis in liver transplant surgery, ${ }^{1}$ showed at that time that these devices are beneficial in many ways: to summarize, potential added value for patients is because of improved safety (chapter 7), less need for blood testing (chapter 4), earlier and more precise 
diagnosis of coagulation abnormalities (chapters 3, 5, and 6), and shorter length of stay on the ICU and in the hospital (chapter 7). While for hospitals, there is benefit because of similar reasons as for patients. On top of that, overall costs are reduced (chapters 4 and 7 ).

The improvements in patient safety and lower costs for hospitals are the resultant of earlier and more precise diagnosis of coagulation disturbance and the subsequent initiation of adequate therapy by physicians. Other healthcare workers, like those working on laboratories have less workload because of the need for less laboratory tests, the need for less samples which need to be investigated, and the potential to incorporate our measurements in a fully automatic process. Blood bankers might need less allogeneic transfusion products in their stock (chapter 7). In 2016 the WHO released their 'Global Status Report on Blood Safety and Availability", which reported that around $6 \%$ of all blood products have to be discarded in the high income countries and that in these countries $2.7 \%$ is discarded because of expiry, making expiry the biggest reason for discarding blood products worldwide. In 2012 more than half a million of red blood cell concentrates were transfused in the Netherlands. That would mean that around 37 of these red blood cell concentrates were discarded daily in the Netherlands. Reductions in the use of allogeneic transfusion products in expense of enlargements of allogeneic transfusion products results in less wastage of transfusion products. In the end, less blood donors are needed to supply the demand of transfusion products.

The newly developed fibrinolysis assay and the correction of both platelet function testing device, can be nice opportunities for the manufacturers of these devices to improve their tests or for us to hop along the bandwagon (chapters 3 and 5). Fast and easy whole blood point-of-care fibrinolysis testing is so far a niche market and seems to have great growing potential (chapter 8). Not so long ago, thromboelastometry and multiple electrode aggregometry were two separate whole blood point-of-care devices. Nowadays, both techniques have been merged into one machine, the ROTEM platelet. Manual pipetting is still needed in this all-in-one machine, but the most recent addition as of now, the ROTEM sigma, is a fully automated viscoelastic haemostasis monitoring device 


\section{Chapter 9}

in which little to no user input is needed to perform a complete coagulation check. Platelet function assessment is not present on the ROTEM sigma. Haemonetics, the ROTEM competitor, also has improved their TEG5000 coagulation monitor into an all-in-one solution using microfluid cartridges. The TEG $6 \mathrm{~s}$ includes both viscoelastic analysis as well as platelet function measurements without the need for manual pipetting. The technique of the TEG $6 \mathrm{~s}$ differs from the traditional viscoelastic properties analysis, as now the effect of various radiofrequencies thrown at the blood sample on the motion of the forming clot during the formation and breakdown is analysed: 'Stronger clots have higher resonant frequencies and higher TEG readouts'. ${ }^{3}$ According to Haemonetics ${ }^{4}$ the total coagulation testing market potential in 2014 was estimated at 1.5 billion US dollars, of which $1 / 6^{\text {th }}$ derives from advanced coagulation testing (i.e. viscoelastic and platelet function testing). Haemonetics stated it holds an $86 \%$ market share (in disposable units) of this division. Werfenlife, the healthcare technology corporation specialized in in vitro diagnostics and medical devices which acquired ROTEM in 2016, reports that in vitro diagnostics represent $84 \%$ of their total revenue with almost one billion US dollars in sales. ${ }^{5}$ Their haemostasis portfolio has a constant currency growth of $+6.2 \%$, with a global market share of $28 \%$, ranking it at number one in the world. Our new fibrinolysis assay has the potential to be implemented in the current ROTEM portfolio of tests, specifically targeting the fibrinolytical pathway of haemostasis. Haemonetics, the other partner with whom cooperation can be achieved, has to validate our method on their new resonance based TEG 6s system in order to further develop our tPA induced viscoelastic assay principle.

On the other hand, our established reference intervals for the platelet function measuring devices might be of less interest to the manufacturers of both MEA and PFA-100, because the tests themselves remain untouched. It is only the calculation algorithm of the new reference ranges which need to be incorporated in their existing devices. However, our findings could mean a boost in sales for PFA-100 and MEA, because researchers and clinicians gain new interest after publication of our research paper on how to correctly interpret platelet function in anaemia and thrombocytopenia conditions. 
As of now, around ten $\mathrm{mL}$ of whole blood is needed to globally assess the coagulation status of patients. Whether the panel of blood tests and the results of the interviews can pinpoint the cause of the possible bleeding remains to be seen, as the research on this topic is still on-going. If reduction of the amounts of $\mathrm{mL}$ of blood to just a drop can be achieved, like I speculated in my Dutch assay, also remains to be seen. However, whole blood thrombin generation testing using the calibrated automated thrombogram (CAT), pioneered by Hemker et al, ${ }^{6}$ has already made this step from $\mathrm{mL}$ to drops. ${ }^{7}$ Monitoring of anticoagulant medication might emerge to be the unique selling point (USP) of whole blood thrombin generation testing using CAT.

When we think about investments of the future, we see other start-ups emerge from everywhere (e.g. Stasys Medical Corp ${ }^{8}$ has developed a patented microfluid platelet aggregation and contractility method under shear stress; Emulate Inc. ${ }^{9}$ pioneered the Organs-on-Chips technology; et cetera) which all present 'new technology' to investigate haemostasis using drops of blood. Seeing how far TEG and ROTEM have become, these start-ups might be wandering in a snake pit. Their USP should gravely outweigh those of TEG and ROTEM, as well as the field of antiplatelet and anticoagulant medication monitoring. In my opinion implemented point-of-care whole blood haemostasis monitoring technology should address the full range of haemostasis: from start of coagulation to breakdown of the clot. It should give an answer to the most essential question: if a patient is bleeding, is it because of a disturbed haemostasis balance or not. It should point clinicians to the cause or to the potential culprit would haemostasis be deranged. It should help clinicians decide which treatment strategy to initiate: focus on platelets, coagulation factors, or fibrinolysis. Foremost, it should be fast, compact, and easy to use and maintain. The viscoelastic methods of TEG and ROTEM seem to embody this whole concept the best and will probably remain the most important players on this point-of-care market. On the other hand, for start-ups to become successful they sometimes need to think outside the box and might have to introduce something which creates demand instead of fulfilling current ones. 


\section{Chapter 9}

\section{References}

1. Kang YG, Martin DJ, Marquez J, Lewis JH, Bontempo FA, Shaw BW Jr, Starzl TE, Winter PM. Intraoperative changes in blood coagulation and thrombelastographic monitoring in liver transplantation. Anesth Analg. 1985 Sep;64(9):888-96.

2. World Health Organization. Global Status Report on Blood Safety and Availability 2016. 2017. ISBN 978-92-4-156543-1. File: 'WHO.2016-9789241565431-eng.pdf'. Available from: http://apps.who.int/iris/bitstream/10665/254987/1/9789241565431-eng.pdf

3. Gurbel PA, Bliden KP, Tantry US, Monroe AL, Muresan AA, Brunner NE, Lopez-Espina CG, Delmenico PR, Cohen E, Raviv G, Haugen DL, Ereth MH. First report of the point-of-care TEG: A technical validation study of the TEG-6S system. Platelets. 2016 Nov;27(7):642-649.

4. Gould G. Innovation and Automation for Blood Management. November 20th, 2014. File: 'TH 08403 Haemonetics FINAL.pdf'. Available from:

http://www.jefferies.com/CMSFiles/Jefferies.com/files/TH\%200840\%203\%20Haemonetics\%20F INAL.pdf

5. Werfenlife. Annual Report 2016. File: 'annualReport_2016_v5.pdf'. Available from: http://www.werfenlife.com/werfenlife/flipbook/annualReport_2016_v5.pdf

6. Hemker HC, Giesen P, AIDieri R, Regnault V, de Smed E, Wagenvoord R, Lecompte T, Béguin $\mathrm{S}$. The calibrated automated thrombogram (CAT): a universal routine test for hyper- and hypocoagulability. Pathophysiol Haemost Thromb. 2002 Sep-Dec;32(5-6):249-53.

7. Ninivaggi M, Apitz-Castro R, Dargaud Y, de Laat B, Hemker HC, Lindhout T. Whole-blood thrombin generation monitored with a calibrated automated thrombogram-based assay. Clin Chem. 2012 Aug;58(8):1252-9.

8. Stasys Medical Corp. 2017. Webpage: https://www.stasysmedical.com

9. Emulate, Inc. 2017. Webpage: https://emulatebio.com 


\section{Chapter 10}

\section{Acknowledgements}





\section{Chapter 10}

\section{Acknowledgements}

Throughout the years it became apparent that starting and finishing up a PhD is not something which is done on your own. In the end the list is limitless of all who have supported me. As such it seems impossible to acknowledge everybody personally, however a few have to be mentioned here explicitly.

First, I have to acknowledgements Prof. ten Cate and all of the biochemistry department at the Maastricht University with helping me do research in this awesome and exciting field of coagulation. Dear Prof. ten Cate, dear Hugo, thank you for giving me the opportunity of starting my research at the Laboratory for Clinical Thrombosis and Haemostasis. The finalisation of this dissertation has taken some time but by writing this chapter it feels complete. To everybody from biochemistry who helped me by giving input on my work, with whom I had lovely days out, with whom I went on congresses abroad, with whom I went for a pub quiz: thank you!

Prof. Buhre, dear Wolfgang, thank you for your thorough input on my dissertation. You asked me not to have an elaborate dissertation with too many chapters through which the readers had to struggle. By putting the focus back into it and reducing parts to its essence, I think we are successful. Furthermore I appreciate your advice and help aiming for a fellowship abroad. I hope to work again with you in the future either doing research, clinical work, or both.

Dr. Henskens, dear Yvonne, we had quite a lot of fruitful discussions not only about the research from this thesis but also about other studies in which we were involved. I admire your dedication in doing haemostasis research, bringing colleagues together from different disciplines, and bridging the gap between basic science, laboratory science and clinical science. In the end, we both can 
be proud of the work presented here and probably that of the future. Thank you for keeping faith in me.

Dr. Lancé, dear Marcus, I vividly remember the first time we met in your office before starting my research participation in medicine. You were and still are enormously passionate about doing research in haemostasis and this was apparent from to moment we met. You are full of ideas and always, literally always, seem to have time to discuss research. I have to thank you for all your guidance, your feedback, but also for getting me in touch with your colleagues in the field of haemostasis abroad. We surely keep in touch because we have to keep haemostasis research moving.

A special thank you goes out to all of my (former) colleagues of the anaesthesiology department in Maastricht. I want to thank all who helped me in my research but also those with whom I had fun together the past years. I greatly appreciate the drinks and snacks (or even diners) at Café Thembi and foremost the barbeque parties we had at my place. We will surely meet each other in the future, let it be in Toronto or Maastricht.

My two paranymphs, Ralph and Najat. You both are such nice colleagues and friends, that you need to be praised. Dear Ralph, we have known each other for years now, starting all the way back from the beginning of our medicine studies. We have had some awesome times together not only in Sittard, Maastricht and Eindhoven but also in Geel, Belgium, in Ferrara, Italy, and in New York, the USA. That we both ended up becoming anaesthesiologists, cannot be a coincidence. Dear Najat, I have the deepest respect for you. Nothing seems to hold you back in succeeding in life. Besides that, I really enjoy your company and laughter at work, but also when we were on international meetings in New Orleans, the USA, and in Dubai, the UAE. That is why I want to thank you both for your time. I wish you both the best of luck for the rest of your lives. You are invited to come over to Toronto any time. 
My family is the most important possession I have in my life; something that no money can buy. They have always supported me in reaching my goals and therefor I must thank especially my parents, my siblings, and my grandfather for everything they helped me with in my life.

Marion 'Moekiepoekie', dear mom and cooking companion. Your guidance seems to be the common thread throughout my whole life. You have given me balance (homeostasis) in my life and in my studies. By shortly interrupting both of them with an STD (i.e. Studying: Temporary Discontinued, or in Dutch, SOA: Studie Onderbrekende Activiteit) in order to shop for groceries, to buy a barbeque, and then do elaborate cooking, you kept me on track. You embodied what is necessary in having a cosy family. Soon things will change and having a break or cooking together will be a bit problematic when I am over in Canada. I am going to miss your hearty home, your presence and your warmth. Furthermore, I am going to miss (y)our humour. For the future: please keep listening to your heart (literally and figuratively speaking) and enjoy life to the fullest here in the Netherlands and in France. My invitation for going on holidays to see the Aurora Borealis is still active and I hope we can cross it off our list someday. Love you!

Gérard 'Paps', dear dad and pater familias. You were always working hard and late. I now appreciate all the things you have done for me and our family. Thank you for giving me the opportunity and support to continue studying. You were the one who convinced the principal of high school that I should keep getting English classes. In the end this worked out for the best. That you were asked to give a PowerPoint presentation at our first-year ceremony for medicine was quite a surprise, although nowadays I would not like to see any more baby photos of me in public. Our first New York trip after finishing high school may have been the beginning of me getting infected by the traveling bug. I hope that our latest trip we had together to India will certainly not be the last one. After finishing this thesis, I will start working on a nice photo book for all to see. For now, I 


\section{Chapter 10}

think we should have a nice glass of white port wine together to celebrate the ending of an era and the start of a new one. Salut!

Margôt "Gootje" and Jurriaan "Jurr", my dearest little sister and little brother. I love you both very much and I hope that you will also succeed in everything you would like to have happen in your lives. Margôt, I admire your artistic capabilities and perseverance, which will surely help you obtain all your ambitions in work and in life. Jurriaan, your background in hospitality can give you endless possibilities. Take full advantage of that on every occasion. My advice for both of you: never stop dreaming, set your goals and work hard, do whatever you like to do, but say no if you have to. Keep remembering:

"Do as you would be done by."

("Wie goed doet, goed ontmoet.").

Love you with all my heart!

Jos, dear grandpa. Your laudation at my graduation ceremony for medicine was quite a statement. In my experience it was applauded by everybody present at that time. I think that perseverance in reaching ones goals in life is something I got taught from you. In the end, you asked me to hurry up, because you and grandma wanted to be present at my $\mathrm{PhD}$ promotion. Unfortunately grandma has passed away meanwhile, but she resides in everybody's heart. And one thing that I will never forget is the Dutch quote hanging in your old apartment complex, summing up everything what is important having in life:

"Met stenen bouwt men een huis, met liefde een thuis."

("Home is where the heart is.")

Salut! 
In the end, some things you have to do on your own and literally writing these final words of this dissertation is just one of those. As such, as my final remark, I want to thank all the rest of my family and all who have passed away before me over the years for their companionship.

Thank you and all the best! 



\section{Chapter 11}

\section{Curriculum vitae}





\section{Chapter 11}

\section{Curriculum vitae}

Gerhardus Johannes Albert Josef Maria (Geert-Jan) Kuiper was born on the $14^{\text {th }}$ of June 1983 in Maastricht, the Netherlands.

In 2001 he completed his pre-university secondary education (Gymnasium) at the Trevianum Scholengemeenschap in Sittard, the Netherlands.

Afterwards he studied Medicine for two years at the Catholic University of Leuven, Belgium; studied one year of Health Sciences at the University of Maastricht and later restarted studying Medicine in 2004 at the University of Maastricht, the Netherlands. He completed his medical degree in 2010.

In his last year of Medicine he was already involved in thrombosis and haemostasis research let under the supervision of Dr. Marcus D. Lancé. After finishing his medical degree, he was appointed a research position at the Laboratory of Clinical Thrombosis and Haemostasis by Prof. Dr. Hugo ten Cate at the Cardiovascular Research Institute Maastricht (CARIM), University of Maastricht. In December of 2012, after a little over two years of research, he started his anaesthesiology residency at the Maastricht UMC+ ending in November of 2017, while finishing his PhD program. Starting July 2018 he has acquired a fellowship in Advanced Clinical Practice at the Toronto General Hospital, Toronto, Canada. 



\section{Chapter 12}

\section{Scientific output}

Publications

Book chapters

Posters and abstracts Oral presentations and awards

(in reverse chronological order) 



\section{Chapter 12}

\section{Scientific output}

Publications

Kuiper GJ, Christiaans MH, Mullens HJ, ten Cate H, Hamulýak K, Henskens YM. Routine haemostasis testing before transplanted kidney biopsy: a cohort study. Transpl Int. 2017 Nov 6.

Kuiper GJ, Houben R, Wetzels RJ, Verhezen PW, van Oerle R, ten Cate H, Henskens YM, Lancé MD. The use of regression analysis in determining reference intervals for low hematocrit and thrombocyte count in Multiple Electrode Aggregometry and Platelet Function Analyser 100 testing of platelet function. Platelets. 2017 Jan 9:1-8.

Kuiper GJ, Henskens YM. Rapid and Correct Prediction of Thrombocytopenia and Hypofibrinogenemia with Rotational Thromboelastometry in Cardiac Surgery Reconsidered. J Cardiothorac Vasc Anesth. 2016 Dec;30(6):e55-e56.

Van Poucke S, Stevens K, Wetzels RH, Kicken C, Verhezen PW, Theunissen HM, Kuiper GJ, van Oerle R, Henskens YM, Lancé MD. Early platelet recovery following cardiac surgery with cardiopulmonary bypass. Platelets. 2016 Dec;27(8):751-757.

Kuiper GJ, Kleinegris MC, van Oerle R, Spronk HM, Lancé MD, Ten Cate H, Henskens YM. Validation of a modified thromboelastometry approach to detect changes in fibrinolytic activity. Thromb J. 2016 Jan 14;14:1.

Olde Engberink RH, Kuiper GJ, Wetzels RJ, Nelemans PJ, Lancé MD, Beckers EA, Henskens YM. Rapid and correct prediction of thrombocytopenia and hypofibrinogenemia with rotational thromboelastometry in cardiac surgery. J Cardiothorac Vasc Anesth. 2014 Apr;28(2):210-6.

Ninivaggi M, Feijge MA, Baaten CC, Kuiper GJ, Marcus MA, Ten Cate H, Lancé MD, Heemskerk JW, van der Meijden PE. Additive roles of platelets and fibrinogen in whole-blood fibrin clot formation upon dilution as assessed by thromboelastometry. Thromb Haemost. 2014 Mar 3;111(3):447-57.

Lancé MD, Kuiper GJ, Sloep M, Spronk HM, van Oerle R, ten Cate H, Marcus MA, Henskens YM. The effects of pneumatic tube system transport on ROTEM analysis and contact activation assessed by thrombin generation test. Thromb Res. 2012 Sep;130(3):e147-50. 
Kuiper GJ. Case report: Minor surgery, major bleeding. American Austrian Foundation Online Case Library, Case Presentations from Fellows of the Salzburg Medical Seminars. 2012 July $19^{\text {th }}$. Available at: http://www.aafonline.org/php/member_area/onlinecases/index.php?act=view\&id=934

Lancé MD, Ninivaggi M, Schols SE, Feijge MA, Oehrl SK, Kuiper GJ, Nikiforou M, Marcus MA, Hamulyak K, van Pampus EC, ten Cate $\mathrm{H}$, Heemskerk JW. Perioperative dilutional coagulopathy treated with fresh frozen plasma and fibrinogen concentrate: a prospective randomized intervention trial. Vox Sang. 2012 Jul;103(1):25-34.

Kilinç E, Schulz H, Kuiper GJ, Spronk HM, ten Cate H, Upadhyay S, Ganguly K, Stoeger T, Semmler-Bhenke M, Takenaka S, Kreyling WG, Pitz M, Reitmeir P, Peters A, Eickelberg O, Wichmann HE. The procoagulant effects of fine particulate matter in vivo. Part Fibre Toxicol. 2011 Mar 15;8:12; author reply 12.

Kuiper GJ, Lancé MD, Smit-Fun VM, Peeters LL, Marcus MA. Platelet monitoring follow-up in a pregnant patient with HELLP syndrome. Platelets. 2011;22(2):160-3.

\section{Book chapters}

Kuiper GJ, ten Cate H. Section 11: The Hematological System, Part 11.1: Laboratory Monitoring, Chapter 266: Coagulation Monitoring. Oxford Textbook Of Critical Care, Second Edition, May 2016, ISBN 9780199600830

Posters and abstracts

Kuiper GJ, Mullens MH, Verhezen PJ, Hamulyak K, Christiaans MH, ten Cate $\mathrm{H}$, Henskens YM. Prediction of bleeding complications after renal biopsy in post-renal transplant patients with uremia. ISTH 2015 Congress, June 20 - 25, 2015, Toronto, Canada.

Henskens YM, van Egmond L, Wetzels RH, Theunissen HM, van Willigem W, Kuiper GJ and Lancé MD. Prospective Hemostasis Registry of Patients in Cardiac Surgery (HEROES-CS): evaluation of the introduction of a thromboelastography based transfusion protocol in cardiac surgery. ISTH 2015 Congress, June 20 - 25, 2015, Toronto, Canada.

Strijkers RH, Kuiper GJ, van Oerle R, Wetzels RH, ten Cate A, ten Cate H, Wittens $\mathrm{CH}$, Henskens $\mathrm{Y}$. Clinically relevant discrepancies between fibrinogen analysis methods in patients undergoing thrombolytic therapy for acute iliofemoral deep venous thrombosis. SSC 2014 Congress, June 23 - 26, 2014, Milwaukee, United States of America. 
Kuiper GJ, Houben R, Verhezen WM, Lancé MD, Christiaans MH, ten Cate H, Henskens YM. Point-of-care platelet testing accurately displays platelet dysfunction in chronic renal insufficiency, but fails to predict bleeding after kidney biopsy. ISTH 2013 Congress, June 29 - July 4, 2013, Amsterdam, The Netherlands.

Olde Engberink HG, Kuiper GJ, Wetzels RH, Nelemans PJ, Lancé MD, Beckers AM, Henskens YMC. Assessment of rotational thromboelastometry in cardiac surgery: correct prediction of clinically relevant thrombocytopenia and hypofibrinogenaemia after 5 min. ISTH 2013 Congress, June 29 - July 4, 2013, Amsterdam, The Netherlands.

Olde Engberink HG, Kuiper GJ, Lancé MD, Henskens YM, Beckers AM. Tissue plasminogen activator induced fibrinolyis on rotational thromboelastometry in chemotherapy-induced thrombocytopenia. ISTH 2013 Congress, June 29 - July 4, 2013, Amsterdam, The Netherlands.

Ninivaggi M, Feijge MA, Kuiper GJ, Baaten C, Marcus MA, ten Cate H, Lancé MD, Heemskerk JW, van Der Meijden PE. Principal roles of platelets and fibrinogen in whole-blood fibrin clot formation in dilutional coagulopathy determined by thromboelastometry. ISTH 2013 Congress, June 29 - July 4, 2013, Amsterdam, The Netherlands.

Swieringa F, Kuiper GJ, Lancé MD, Heemskerk JW, van der Meijden PE. Thrombus formation under flow conditions is enhanced after desmopressin treatment in patients with postoperative bleeding complications. BSHT/UK platelet group meeting, October 18, 2012, Bath, United Kingdom.

Kuiper GJ, Emam LS, Theunissen HM, Lancé MD, Marcus MA. Hemostasis Registry of Patients in Cardiac Surgery (HEROES-CS): Perioperative Risk Factors for Red Blood Cell Transfusion Among Cardiac Surgery Patients. ASA 2012 Congress, October 13 - 17, 2012, Washington, DC, United States of America.

Swieringa F, Kuiper GJ, Lancé MD, Verheijen L, Hughes KR, Marcus MA, Heemskerk JW, van der Meijden PE. Desmopressin treatment improves wholeblood thrombus formation in patients undergoing cardiothoracic surgery. EUPLAN Platelet Conference, September 19 - 21, 2012, Maastricht, The Netherlands.

Kuiper GJ, Van Oerle R, Kleinegris MC, Spronk HM, Lancé MD, ten Cate H, Henskens YM. Technical and clinical validation of a modified thromboelastometry approach on a ROTEM device for the identification of patients at risk for hypo- and hyperfibrinolysis. SSC 2012 Congress, June 27 - 30, 2012, Liverpool, United Kingdom. 
Kuiper GJ, Lancé MD, Sloep M, Spronk HM, van Oerle R, Henkens YM, Marcus $\mathrm{MA}$, ten Cate $\mathrm{H}$. The influence of pneumatic tube system vs. manual transport on activation of coagulation measured with thromboelastometry and thrombin generation. ISTH 2011 Congress, July 23 - 28, 2011, Kyoto, Japan.

Ninivaggi M, Lancé MD, Kuiper GJ, Schols SE, Feijge MA, Marcus MA, ten Cate $\mathrm{H}$, Heemskerk JW. Restoration of impaired hemostasis by platelets and coagulation factors in patients undergoing cardiothoracic surgery. ISTH 2011 Congress, July 23 - 28, 2011, Kyoto, Japan.

Hughes KR, Verheijen L, Feijge MA, Kuiper GJ, Lancé MD, Heemskerk JW. Effects of desmopressin on hemostatic platelet function in patients undergoing cardiothoracic surgery. CARIM 2011 Symposium, November 9 2011, Maastricht, The Netherlands.

Ninivaggi M, Lancé MD, Kuiper GJ, Schols SE, Feijge MA, Marcus MA, ten Cate $\mathrm{H}$, Heemskerk JW. Roles of platelets and coagulation factors in the restoration of impaired hemostasis in patiets undergoing cardiothoracic surgery. Nottingham Platelet Conference, July 15 - 16, 2010, Nottingham, United Kingdom.

\section{Oral presentations and awards}

Co-awarded at the 2018 VHL/PAOKC symposium Bloedtransfusie Ins \& Outs, Utrecht, The Netherlands the VHL samenwerkingsprijs 2018, worth $€ 500$. Februari 8 2018, Utrecht, The Netherlands.

Invited speaker at Danube University Krems (DUK) - Weill Cornell Medicine Qatar (WCM-Q) Seminar on Perioperative Bleeding Management 2017. Topics: Diagnostic Tools: Viscoelastic Tests \& POC Platelet Function Monitoring; Transfusion: Plasma. September 29 - October 3, 2017, Doha, Qatar.

Invited speaker at Danube University Krems (DUK) Seminar on Severe Bleeding Management, Patient Blood Management 2017. Topics: Hemostatic Agents: Tranexamic Acid, Desmopressin, Wound Dressings, et al; Hemostatic Agents: Platelets; Thrombin Generation Tests; Bleeding Management in Peri-partum Hemorrhage. September 4 - 8, 2017, Hainburg, Austria.

Invited speaker at Danube University Krems (DUK) Seminar on Severe Bleeding Management, Patient Blood Management 2016. Topics: Hemostatic Agents: Tranexamic Acid, Desmopressin, Wound Dressings, et al; Perioperative Antiplatelet Therapy; Bleeding Management in Peri-partum Hemorrhage. December 5 - 9, 2016, Hainburg, Austria. 
Invited speaker at Danube University Krems (DUK) Seminar on Severe Bleeding Management, Patient Blood Management 2016. Topics: Diagnostic Tools: Conventional Lab Tests; Diagnostic Tools: Viscoelastic Tests; Hemostatic Agents: Tranexamic Acid, Desmopressin, Wound Dressings, et al. November 7 - 11, 2016, Hainburg, Austria.

Invited speaker at Danube University Krems (DUK) Seminar on Severe Bleeding Management, Patient Blood Management 2016. Topics: Diagnostic Tools: Viscoelastic Tests; Hemostatic Agents: Tranexamic Acid, Desmopressin, Wound Dressings, et al. September 5 - 9, 2016, Hainburg, Austria.

Moderator at XXIV congress of the ISTH and 59th Annual SSC Meeting 2013, Amsterdam, The Netherlands. Session PB 3.22: Platelets: Point-of-care tests. June 29 - July 7, 2013, Amsterdam, The Netherlands.

Awarded at the 2nd yearly day of science at Maastricht UMC+, Maastricht, The Netherlands a 3rd prize for best poster presentation, worth $€ 125$. March 6, 2013, Maastricht, The Netherlands.

Awarded at the Salzburg Weill Cornell, MUW Seminar in Sever Bleeding Management, Salzburg, Austria an Excellent Case Presentation: 'Minor Surgery, Major Bleeding'. July 15 - 21, 2012, Salzburg, Austria.

Awarded at the 58th Annual Meeting of the Scientific \& Standardization Committee (SSC) of the International Society on Thrombosis and Haemostasis (ISTH), Liverpool, United Kingdom a Young Investigator Award 2012, worth \$1000. June 27 - 30, 2012, Liverpool, United Kingdom.

Co-awarded at the 2012 Convention of the Dutch Society of Anesthesiology, Maastricht, The Netherlands a 3rd prize for best publication at the 2012 Convention of the Dutch Society of Anesthesiology, worth €1000. May 10 - 11, 2012, Maastricht, The Netherlands.

Awarded at the Dutch Heart Foundation PhD-training course 'Vascular Biology', Arnhem, The Netherlands a 2nd prize 'Workshop popular science communication'. October 10 - 14, 2011, Arnhem, The Netherlands.

Moderator at the 2011 symposium of the Dutch Society of Thrombosis and Haemostasis, Koudekerke, The Netherlands. Session V: Clinical aspects of haemorrhagic and thrombotic disorders. May 17 - 18, 2011, Koudekerke, The Netherlands. 
